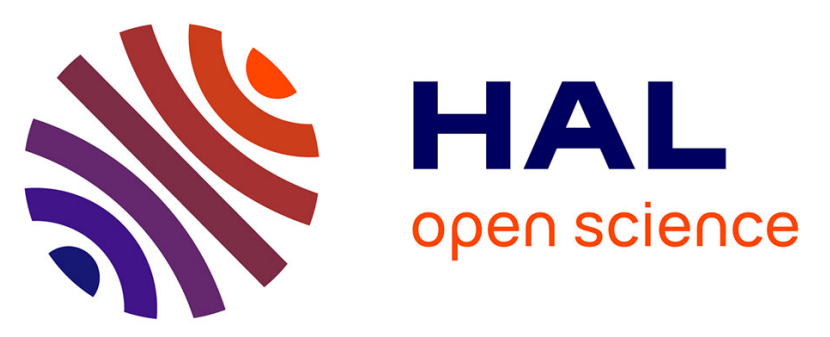

\title{
Les coquilles marines de la nécropole mésolithique de La Vergne (Saint-Jean-d'Angély, Charente-Maritime, France) : de leur collecte à la mise en scène des corps humains
}

Luc Laporte, Catherine Dupont, Yves Gruet, Patrice Courtaud, Henri Duday, Laurent Quesnel, Jean-Yves Lefeuvre, Flavien Bambagioni, Vincent Miailhe, Karine Robin

\section{- To cite this version:}

Luc Laporte, Catherine Dupont, Yves Gruet, Patrice Courtaud, Henri Duday, et al.. Les coquilles marines de la nécropole mésolithique de La Vergne (Saint-Jean-d'Angély, Charente-Maritime, France) : de leur collecte à la mise en scène des corps humains. Gallia Préhistoire - Préhistoire de la France dans son contexte européen, 2021, 61 (61), 10.4000/galliap.2160 . hal-03277662

\author{
HAL Id: hal-03277662 \\ https://hal.science/hal-03277662
}

Submitted on 13 Dec 2021

HAL is a multi-disciplinary open access archive for the deposit and dissemination of scientific research documents, whether they are published or not. The documents may come from teaching and research institutions in France or abroad, or from public or private research centers.
L'archive ouverte pluridisciplinaire HAL, est destinée au dépôt et à la diffusion de documents scientifiques de niveau recherche, publiés ou non, émanant des établissements d'enseignement et de recherche français ou étrangers, des laboratoires publics ou privés.

\section{(ㅇ)(1) $\$$}

Distributed under a Creative Commons Attribution - NonCommercial - NoDerivatives 44.0 


\section{Les coquilles marines de la nécropole mésolithique de La Vergne (Saint-Jean-d'Angély, Charente-Maritime, France) : de leur collecte à la mise en scène des corps humains}

Luc Laporte, Catherine Dupont, Yves Gruet, Patrice Courtaud, Henri Duday, Laurent Quesnel, Jean-Yves Lefeuvre, Flavien Bambagioni, Vincent Miailhe et Karine Robin

1 Avec la découverte de sépultures et son mobilier associé, les vestiges du site de La Vergne constituent un ensemble funéraire tout à fait exceptionnel pour la Préhistoire récente sur la façade atlantique de l'Europe. En France et pour la période du Mésolithique, ils ne trouvent guère de comparaison à ce jour qu'avec ceux fouillés dans la première moitié du vingtième siècle sur les îlots bretons de Téviec et d'Hoëdic (Péquart et al. 1937, Péquart et Péquart 1954); notamment de par l'abondance dans les tombes de coquilles marines percées que l'on ne pouvait interpréter alors autrement que comme autant de grains de colliers. De tels assemblages de pièces préhistoriques étudiées séparément furent souvent présentés comme les vestiges d'un "langage » disparu, pour reprendre un terme cher à Y. Taborin (2004), comme autant de signes disjoints dont la syntaxe ne tiendrait qu'à un fil. Le pouvoir informatif de telles parures est multiple. De nombreuses thématiques peuvent ainsi être abordées comme la description de l'acquisition de la matière première (ramassage sur la plage, échange, recyclage, transmission intergénérationnelle), la confection, la manière dont ces coquilles ont été portées ou utilisées, le symbolisme voire la part d'humanité qu'elles peuvent véhiculer, le rapport entre groupes humains, comme les marqueurs identitaires qu'elles peuvent procurer ou véhiculer (par exemple Claassen 1998, Zilhão 2007, Vanhaeren et d'Errico 2011, Rigaud et al. 2014, Perlès 2018). L'étude des coquilles 
de La Vergne en association avec des restes humains fut alors pour nous l'occasion de tester ce que l'on entend ainsi par parure corporelle, pour les époques préhistoriques.

2 À La Vergne, toutes les matières premières utilisées appartiennent au règne animal, à l'exclusion de tout objet façonné en pierre. Les coquilles constituent de très loin un support privilégié. Aux 3298 objets de parure en coquille il faut également ajouter quelques dents percées. Dans tous les cas, la matière première a été peu ou pas transformée. En ce qui concerne les coquilles, le façonnage des pièces se limite généralement à l'obtention d'une perforation, lorsque cette dernière ne résulte pas d'une action naturelle. Comme tout objet façonné par l'être humain, les éléments de parure rendent compte des savoir-faire techniques et du degré de spécialisation de ceux qui les ont élaborés en fonction de normes conceptuelles pré-établies. La diversité des matières premières sélectionnées ainsi que l'éloignement de leurs lieux de collecte, illustrent le mode d'occupation du territoire comme l'étendue des réseaux d'alliance et d'échanges tissés par ces populations.

Cette étude ${ }^{1}$ porte sur les coquilles présentes dans chacune de ces tombes. Elles furent d'abord identifiées comme autant d'objets de parure corporelle, mais nous avons vu que les fouilleurs avaient eux-mêmes commencé à nuancer cette proposition. La présente étude n'aurait pas été possible sans le soin extrême porté à l'enregistrement des données au cours de cette fouille exemplaire, pourtant réalisée dans des délais particulièrement courts et dans des conditions parfois difficiles. Elle rend hommage à ces travaux de terrain minutieux, en prolongeant le souhait de leurs auteurs : "À l'exception des dentales, dont certains sont emboîtés, toutes ces coquilles sont percées. L'enregistrement détaillé de leur disposition réciproque et l'examen d'éventuelles traces d'usure permettront, sans aucun doute, d'apporter de plus amples informations sur l'agencement originel des parures » (Duday et Courtaud 1998, p. 32).

\section{Travaux de terrain}

4 La fouille préventive de fossés et autres structures en creux de l'âge du Fer, menée en 1995 au lieu-dit la Grande Pièce sur la commune de La Vergne près de Saint-Jeand'Angély en Charente-Maritime (fig. 1), a révélé l'existence de plusieurs sépultures préhistoriques attribuées à la période du Mésolithique. Implantées dans des fosses, ces dernières ont été arasées ou recoupées par les occupations protohistoriques d'une part, et par le décapage mécanique de terres superficielles lié à l'opération archéologique d'autre part (Courtaud et Duday 1995). L'extension de ces vestiges au-delà de l'emprise des travaux d'aménagement, reste inconnue. L'existence d'éventuels niveaux d'occupation contemporains, au-dessus du sol de l'époque, reste tout aussi incertaine ; ce qui amena les acteurs de cette découverte à s'interroger sur la pertinence du terme de nécropole, au vu des éléments disponibles (Courtaud et al. 1999, p. 289). 
Fig. 1 - Localisation du site de la Vergne et distribution des structures archéologiques situées dans le périmètre de l'implantation des tombes mésolithiques $\left(7 \mathrm{~m}^{2}\right)$, au sein des plus vastes décapages d'une fouille préventive. Les structures $3,7,10$, et 11 correspondent aux sépultures mésolithiques. Les structures 1,2 et 12 sont postérieures. Elles correspondent à un grand fossé, à une fosse d'extraction d'argile et à une fosse gauloise dans lesquels des éléments de parure et des os humains remaniés ont été découverts. Le fossé postérieur qui a recoupé certaines de ces structures a été attribué à l'âge du fer.

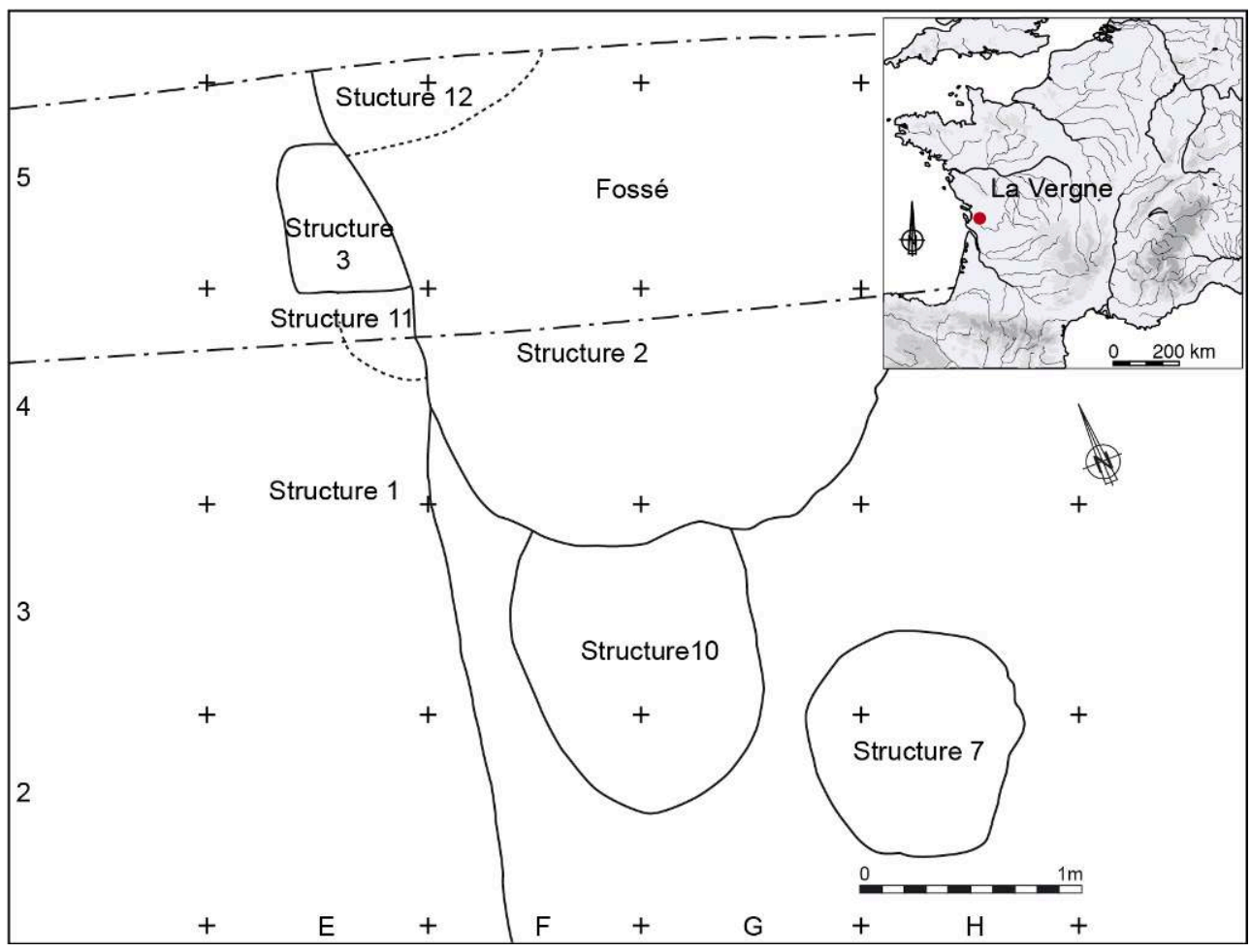

D'après Duday et Courtaud 1998 ; DAO L. Quesnel et C. Dupont.

5 Trois sépultures de La Vergne ont été minutieusement fouillées qui correspondent à autant de fosses distinctes (fig. 2). Quelques ossements humains ou coquilles ocrées ont également été recueillis sur le talus extérieur du fossé, comme dans son remplissage, qui suggèrent l'existence d'autres tombes, entièrement détruites. Les dates radiocarbone disponibles (tabl. 1), effectuées directement sur les ossements humains, sont toutes centrées autour de 8300 av. n.è. (Duday et al. 1998). L'étude des isotopes du carbone et de l'azote concernant les ossements de neuf des individus inhumés suggère une alimentation majoritairement d'origine terrestre (Schulting et al. 2008). Les objets présents dans la fosse, et donc associés à ces différents individus, recèlent en revanche une très grande quantité de coquilles d'origine marine qui font l'objet de cet article. 
Fig. 2 - Vue de la structure 3 à différents stades de la fouille.

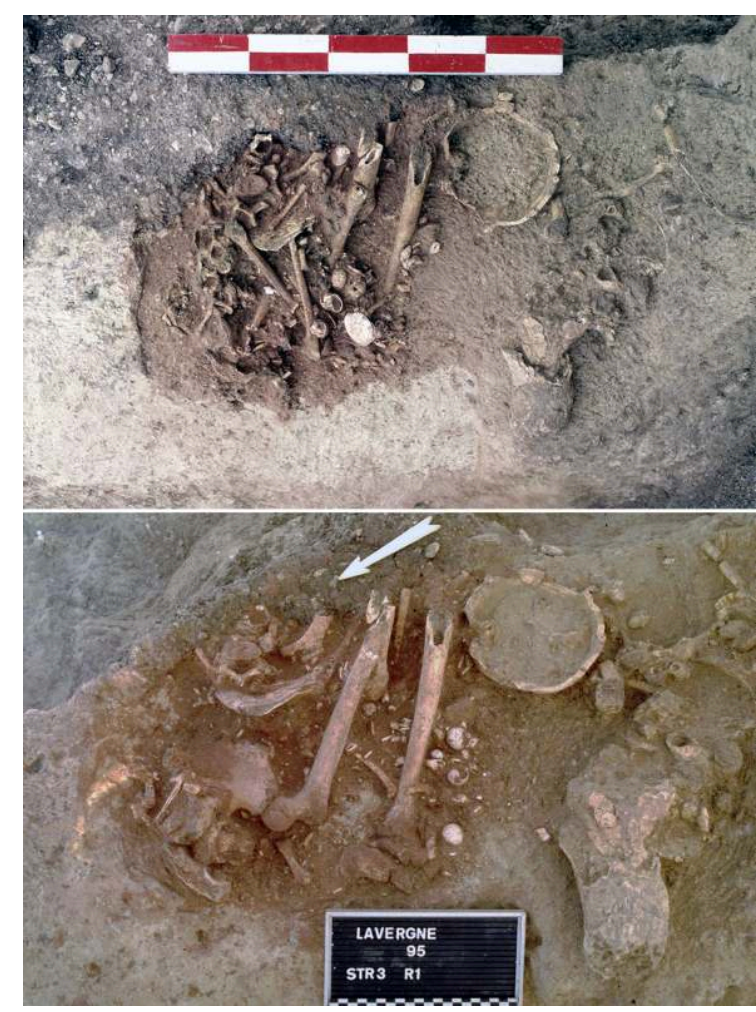

Photos P. Courtaud.

Tabl. 1 - Effectifs ( 15 sujets au total) et datations au radiocarbone ( 6 dates), calibrées à partir d'IntCal20 (Reimer et al. 2020), pour chacune des sépultures mésolithiques étudiées sur le site de La Vergne. La mention « St 10 sujet sup $n^{\circ} 16$ » concerne le squelette d'un adulte très mal conservé (niveau du décapage), déposé sur le dos juste à l'aplomb et au-dessus de la St. 10 qui n'est donc pas ici directement concernée par cette datation.

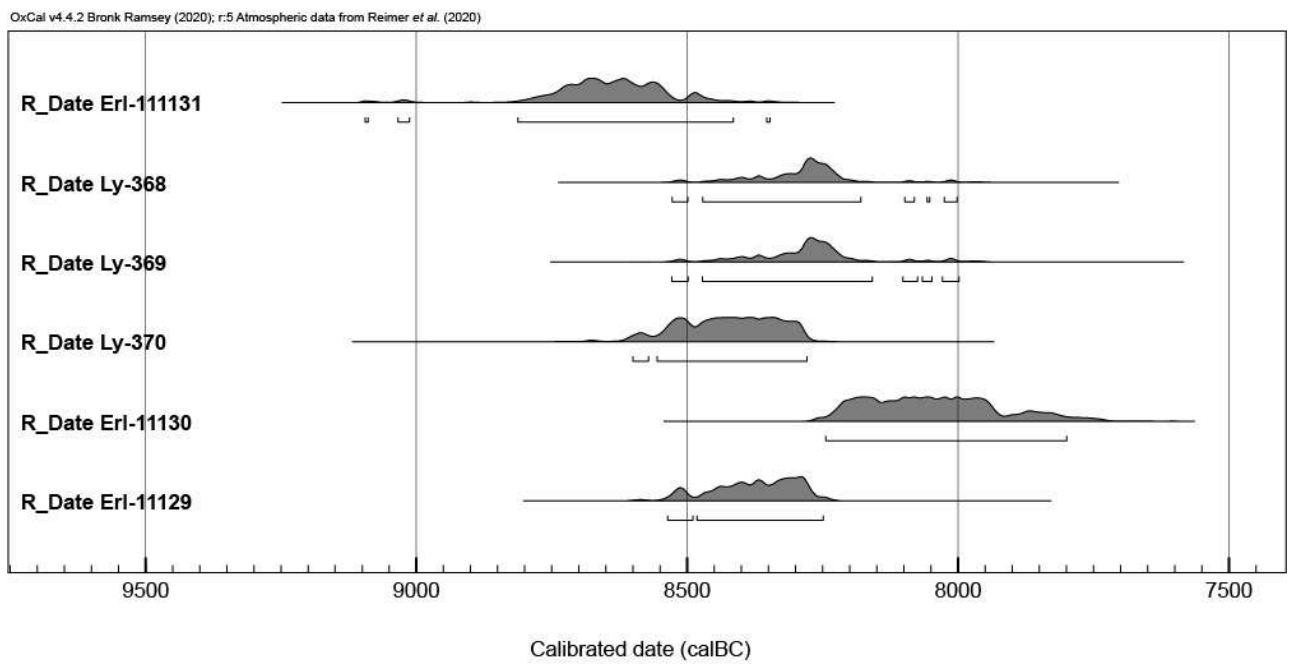

Parmi les sépultures, la fosse 3 n'est que très partiellement connue, car tronquée par des structures postérieures puis arasée par la pelle mécanique (fig. 2). Fort heureusement, c'est aussi la plus profonde des structures attribuées à la période du Mésolithique ancien. Elle n'est conservée que dans sa moitié nord, sur un espace d'environ $0,45 \mathrm{~m}$ sur $0,35 \mathrm{~m}$. Au sein du remplissage de la fosse, les dépôts d'ocre 
forment comme une masse compacte qui englobe les ossements humains. Il s'agit notamment d'une femme déposée en position assise contre la paroi nord de la fosse ; «au fur et à mesure que la destruction des contentions articulaires libérait les pièces osseuses du tronc et de la tête, celles-ci se sont effondrées dans le volume libéré par la disparition des viscères thoraciques et abdominaux » (Duday et Courtaud 1998, p. 33). D'autres pièces ont pu être entraînées par un tel mouvement. En revanche, rien ne subsiste en place de ce dépôt au-dessus des parties distales du fémur ou proximale du tibia, tronquées par la pelle mécanique.

7 Cette fosse contenait également les restes d'un fotus proche du terme, ou d'un enfant mort-né prématuré, et surtout ceux d'un enfant de douze à quinze ans, également déposé en position assise et dont "plusieurs phalanges de la main gauche étaient plaquées contre la face latérale droite de l'ilium droit de la femme » (Duday et Courtaud 1998, p. 33). Il s'agit de sépultures simultanées pour des corps déposés en espace colmaté. Le remplissage contenait, outre de nombreuses coquilles marines percées, plusieurs craches de cerf, des canines de renard et une canine de loup percées. Un fragment de cheville osseuse d'aurochs a été recueilli dans ce qui restait de la partie sud dépourvue d'ocre.

8 La fosse 7, de forme ovale, mesure entre 0,85 et 1,05 $\mathrm{m}$ de large (fig. 3). Elle fut creusée sur au moins $30 \mathrm{~cm}$ de profondeur. À l'exception de son extrémité sud, le remplissage de cette fosse contenait d'importants dépôts d'ocre. Au nord, les squelettes de deux individus ont été dégagés reposant sur le côté gauche en position fortement contractée. Il s'agit de dépôts primaires simultanés, le membre inférieur gauche de l'un des sujets s'appuyant sur la partie postérieure du tronc de l'autre sujet. « Les membres supérieurs sont plaqués contre le thorax, les coudes fléchis et les mains ramenées au niveau du menton. Les cuisses sont elles aussi plaquées contre le tronc, les genoux en flexion maximale " (Duday et Courtaud 1998, p. 28). Si les mandibules sont restées en place, les crânes en revanche ont été détruits au décapage : ils reposaient chacun sur un galet, en quartzite blanc ou en roche métamorphique altérée. Les restes d'un enfant de moins de deux ans, recouverts par quelques os humains brûlés, furent placés dans la fosse en même temps que le corps des deux adultes. Outre plusieurs centaines de coquilles percées et une quarantaine de pièces lithiques, les fouilleurs mentionnent également la présence de canines de renard, de craches de cerf et même plusieurs dents humaines percées. Ils précisent alors que certains de ces dépôts «doivent correspondre à des offrandes plutôt qu'à des ornements portés par les défunts » (Duday et Courtaud 1998, p. 30), ce qui se trouvera largement confirmé par la suite. 
Fig. 3 - Vue générale de la structure 7 en cours de fouille avec un détail sur des nasses alignées.

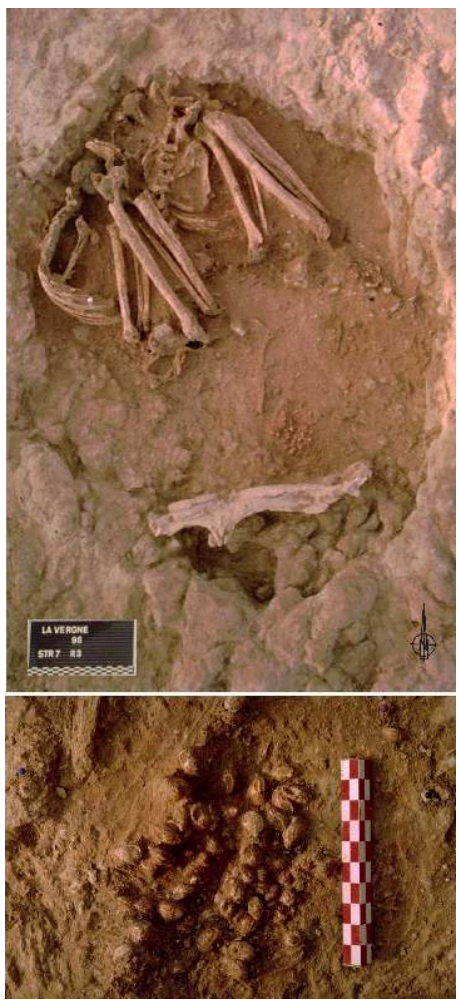

Photos P. Courtaud.

9 À l'ouest de la fosse 7, la structure 10 est divisée en deux parties. La moitié sud, dépourvue d'ocre est occupée par deux massacres d'Aurochs. Le secteur Nord fortement ocré est lui associé à des dépôts humains composés des restes d'un homme et de ceux d'un enfant de 1 à 2 ans derrière le dos de l'adulte. De nombreux coquillages marins sont situés près du menton de l'adulte (fig. 4).

Les vestiges d'autres structures sépulcrales furent également identifiés dont l'attribution à la même période du Mésolithique reste parfois incertaine. La fosse 3 semble ainsi avoir été recoupée par une autre fosse contenant les restes disloqués d'un individu âgé, eux-mêmes surmontés par ceux d'un individu masculin accompagné de quelques ossements appartenant à deux enfants, l'un périnatal, l'autre âgé de cinq à dix ans. Quelques coquilles percées et un fragment de silex ont également été recueillis dans le remplissage (dépourvu d'ocre) de cette structure, intitulée structure 11. La fosse 10 était par ailleurs surmontée par les restes partiels d'un adulte, très endommagés, ne disposant d'aucun dépôt d'ocre ni de coquilles marines (Duday et Courtaud 1998). 
Fig. 4 - Vue générale de la structure 10 en cours de fouille avec un détail sur un lot de coquilles proche du menton d'un adulte.
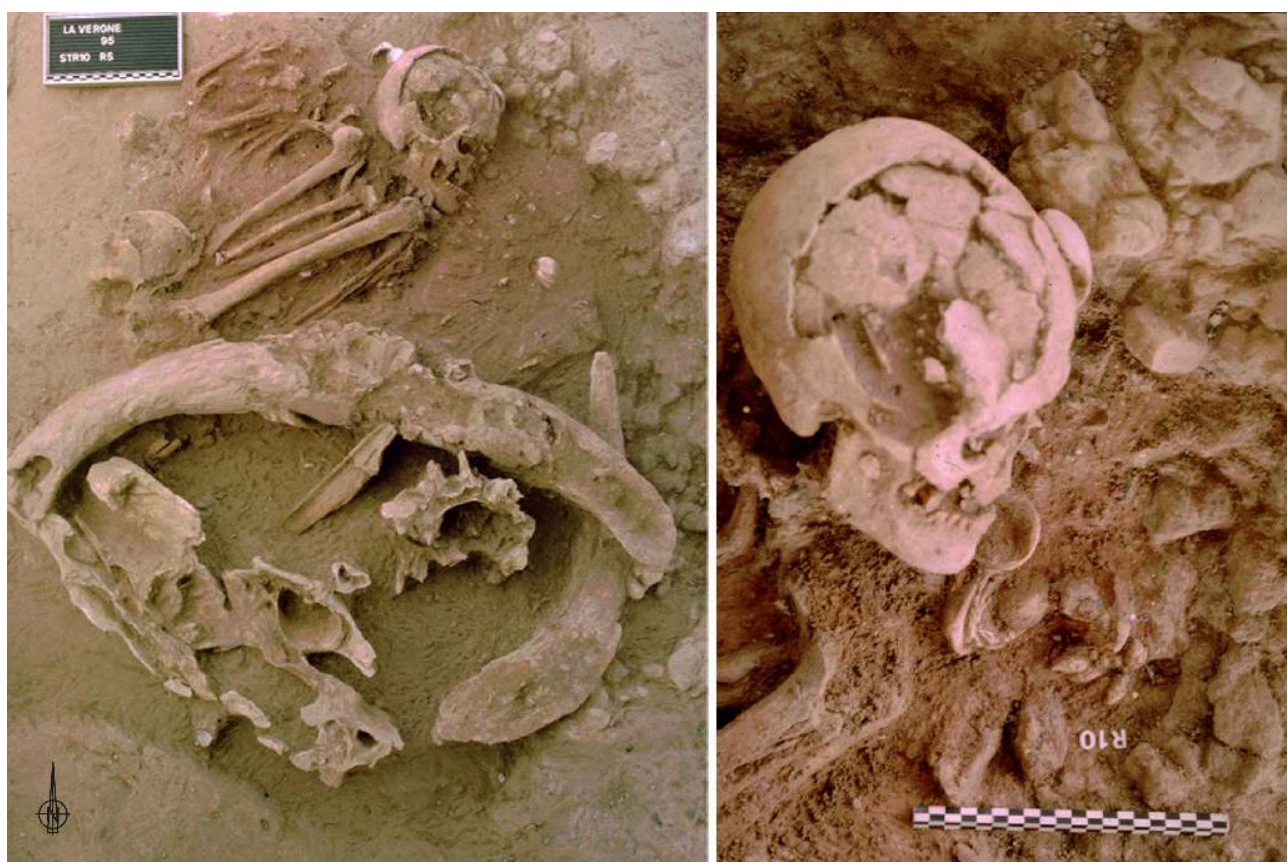

Photos P. Courtaud.

\section{Les coquilles marines}

11 Une deuxième partie de cet article traitera des entités naturelles recueillies au sein de chacune de ces tombes: les coquilles marines. Elle abordera successivement la détermination spécifique de ces mollusques, ainsi que le spectre faunique obtenu à l'échelle de l'ensemble du site. Les espèces déterminées sont un moyen d'avoir accès, aux biotopes des différentes entités naturelles concernées, mais aussi à l'action anthropique par le biais de l'activité particulière qu'est la collecte. Un choix s'opère qui n'est peut-être pas indépendant des processus taphonomiques affectant chaque coquille échouée sur la côte. Les modalités de leur transport vers des contrées plus continentales restent difficiles à appréhender. Au bout du compte, chaque tombe recèle des coquilles aux formes bien différentes dont l'abondance relative permet déjà de commencer à structurer l'information.

Pour commencer, nous nous sommes attardés sur la détermination et la quantification des espèces utilisées. Donner un nom à une espèce n'est pas une fin en soi. Le but est de pouvoir remonter dans la chaîne d'acquisition jusqu'aux biotopes des mollusques utilisés et de caractériser les lieux dans lesquels ils ont pu être trouvés.

Une fois déterminées, les espèces en présence ont été quantifiées. Cette quantification permet de caractériser le spectre malacofaunique du site de La Vergne, aussi bien du point de vue de sa richesse spécifique que des proportions relatives de chaque espèce identifiée. Leur état lors de leur collecte, c'est-à-dire, vivant ou mort, apportera une information complémentaire sur l'organisation de la collecte de coquilles destinées à être transformées en parure par rapport à d'autres activités, voire aux phénomènes des marées. Pour cette étape de l'analyse, une typologie de fragmentation des coquilles a 
été appliquée pour permettre de juger de l'état de la dégradation générale des coquilles avant leur ramassage en bord de mer et lors de leur découverte par les archéologues.

Les données spatiales de ces coquilles permettent ensuite la comparaison de la composition de la malacofaune en fonction des différentes structures du site. La perspective est de savoir s'il existe au niveau de la malacofaune une hétérogénéité similaire à celle observée pour les traitements funéraires (Duday et al. 1998, p. 34). La notion de choix et de différenciation entre structures funéraires sera ainsi abordée.

15 Ces étapes de l'analyse visent donc à retracer les premiers éléments de la chaîne opératoire de la production de parure, à savoir sa recherche et sa collecte.

16 L'intégralité des coquilles prélevées dans la zone fouillée a été étudiée pour cette première étape de l'analyse, soit 3 297. Un lavage, indispensable à la reconnaissance des espèces, a d'abord été effectué. La première difficulté de cette étape réside dans l'hétérogénéité de la dégradation des coquilles. En effet, selon les espèces le test (c.à.d. la coquille) est plus ou moins épais donc plus ou moins solide. Le degré d'altération au sein d'une même espèce est également très varié. D'autre part, les sédiments diffèrent selon les échantillons. À l'échelle du site, l'état de compaction du sédiment variait suivant les structures considérées. Ainsi, comme il avait été noté lors de la fouille, une partie de la structure 3 possédait un sédiment induré (Duday et al. p. 9), ce qui a rendu le lavage difficile. Le choix du nettoyage à l'eau et au pinceau a paru être la méthode la mieux adaptée. Étant donné l'induration du sédiment présent dans les coquilles, cellesci ont subi un trempage préalable dans l'eau. Ceci a permis d'en ôter plus facilement le contenu à l'aide de pinceaux et d'allumettes. Une fois lavés, les échantillons ont été séchés. Ils étaient ensuite prêts à l'observation. Seules quelques coquilles associées entre elles avec du sédiment ont été exclues de ce protocole pour permettre de futures observations sur leur positionnement les unes par rapport aux autres.

Un certain nombre de variables quantitatives et qualitatives ont été retenues dans le cadre de cette étude. Les coquilles ont été quantifiées en nombre de restes (NR) et pesées. Chaque reste décompté représente soit un fragment de coquilles soit un test complet. Plusieurs mesures ont également été effectuées (fig. 5-6). 
Fig. 5 - Mesures réalisées sur les gastéropodes de La Vergne et localisation des perforations (numéro de 1 à 4).

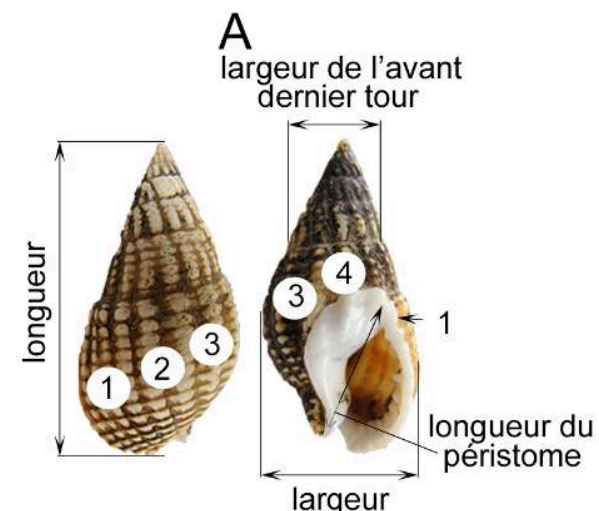

C

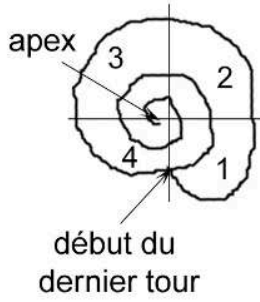

B

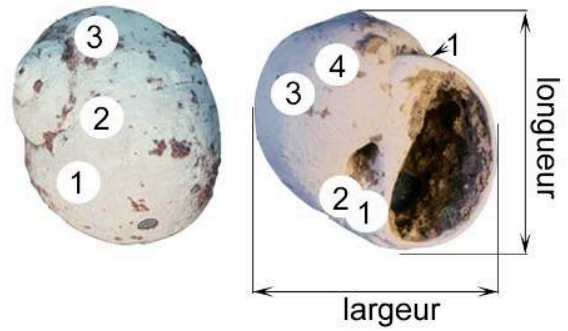

$\mathrm{D}$

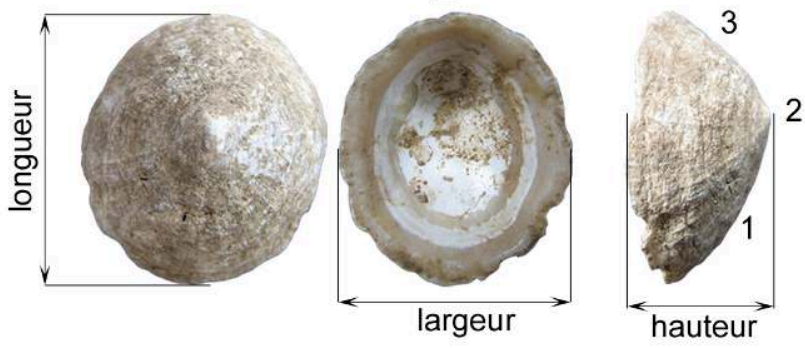

A. Gastéropode turbiné à apex aigu ; B. Gastéropode à apex aplati ; c. Localisation de la perforation ; D. Gastéropode conique.

DAO C. Dupont. 
Fig. 6 - Mesures réalisées sur les scaphopodes et bivalves de La Vergne.
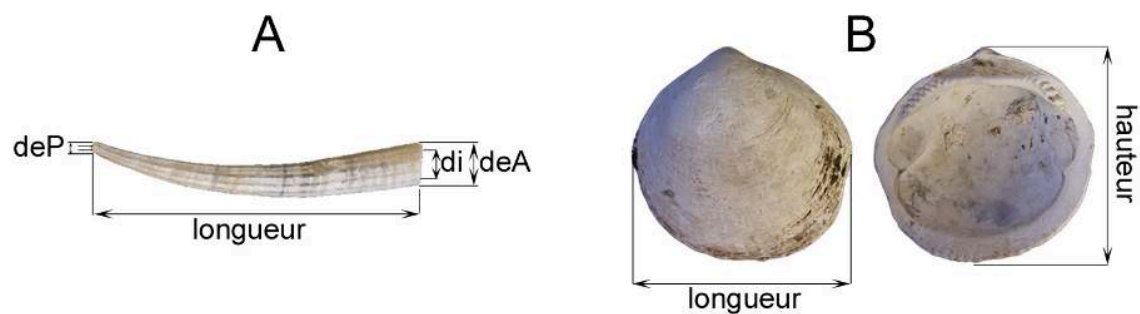

C

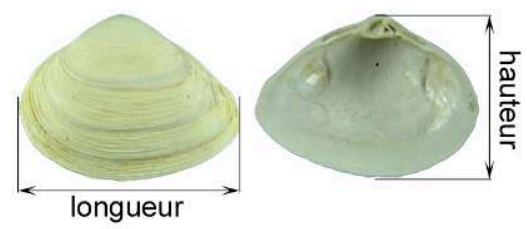

$\mathrm{E}$

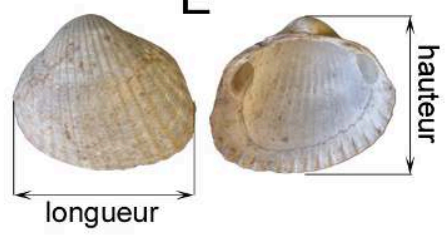

D
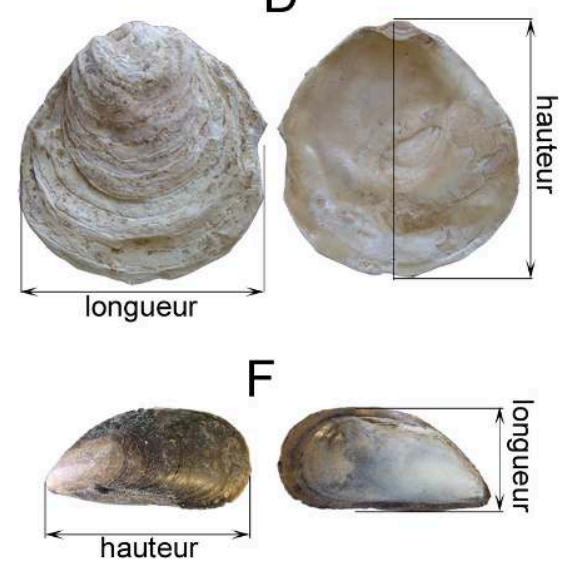

A. Scaphopode ; B. Bivalve intégripallié taxodonte ; c. Bivalves sinupallié hétérodonte ; D. Bivalve de type « Ostréidés »; $\mathbf{E}$. Bivalve intégripallié hétérodonte ; $\boldsymbol{F}$. Bivalve de type « Mytilidés ». DAO C. Dupont.

Les différentes parties anatomiques des coquilles citées dans cet article sont aussi localisées en fonction des mollusques présents à La Vergne (scaphopode, gastéropode à apex aigu ou aplati, gastéropode conique, bivalve hétérodonte, taxodonte, sinupallié ou intégripallié, bivalve "Ostéréidé » ou " Mytilidé »; fig. 7-8). Ces figures permettent de visualiser rapidement les termes utilisés dans ce qui suit en fonction des différentes formes des coquilles, ainsi que l'emplacement des perforations. 
Fig. 7 - Critères descriptifs des gastéropodes de La Vergne.
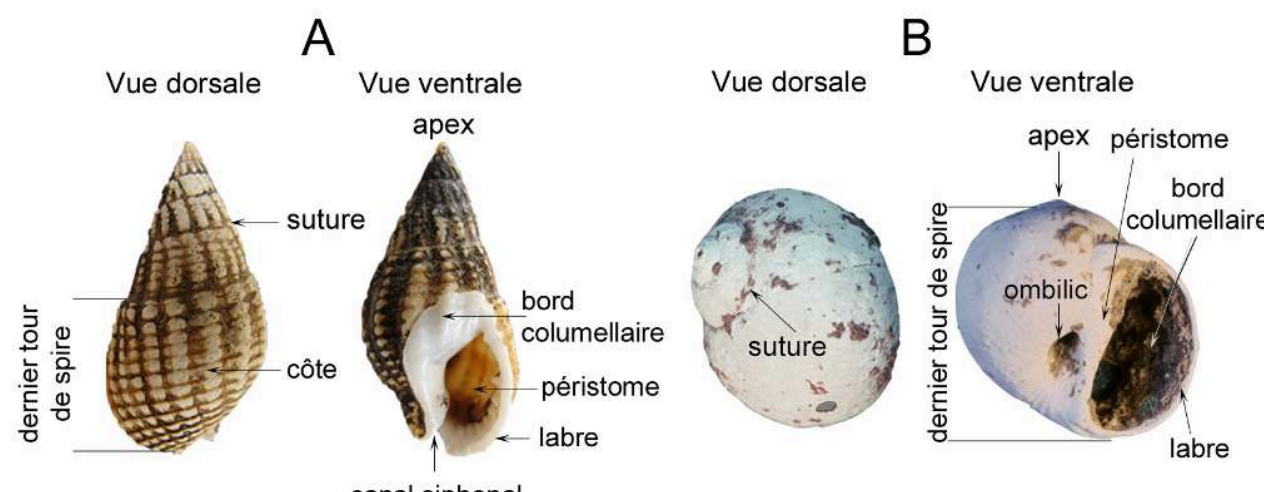

canal siphonal

C

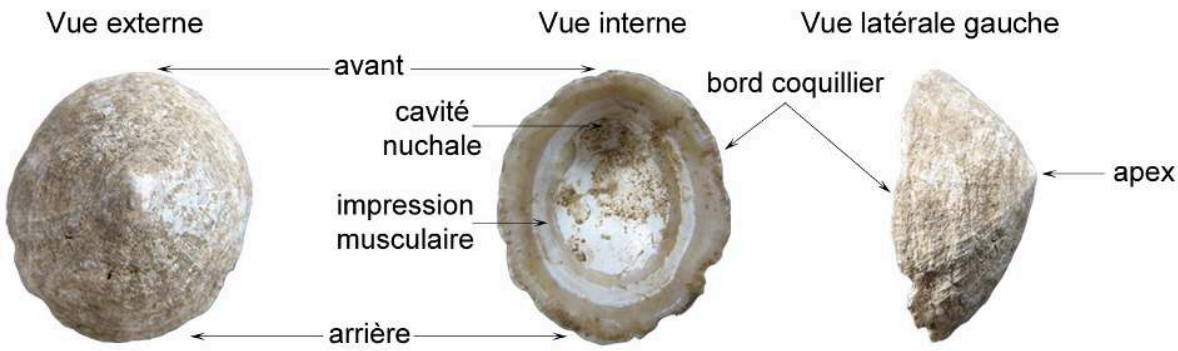

A. Gastéropode turbiné à apex aigu ; B. Gastéropode à apex aplati ; c. Gastéropode conique. DAO C. Dupont 
Fig. 8 - Critères descriptifs des scaphopodes et bivalves de La Vergne.

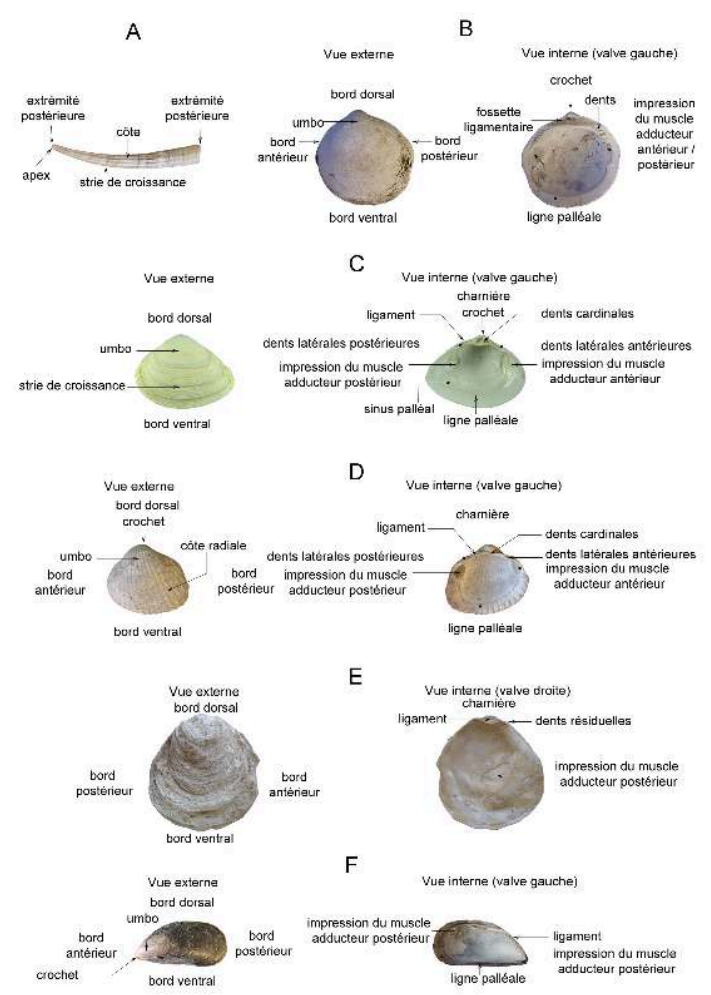

A. Scaphopode ; B. Bivalve intégripallié taxodonte ; c. Bivalves sinupallié hétérodonte ; D. Bivalve de type «Ostréidés »; $\boldsymbol{E}$. Bivalve intégripallié hétérodonte ; F. Bivalve de type « Mytilidés ». DAO C. Dupont.

\section{Action anthropique sur la matière}

L'étude des différentes chaînes opératoires mises en œuvre pour le façonnage d'objets de parure nous renseigne sur l'histoire des techniques et indirectement sur l'identité des personnes impliquées dans ces activités de production. Ce sera le sujet d'une troisième partie de cet article ${ }^{2}$. Un tel niveau d'analyse suppose l'observation des stigmates laissés sur ces milliers de coquilles, pour chaque pièce et pour différentes zones de son anatomie, par les différentes actions techniques qui contribuent à leur façonnage. Parfois, il s'agit du façonnage de la perforation. Plus souvent encore, des traces d'usures sont détectées. Confrontées à la position précise de chaque objet, elles permettront parfois d'émettre quelques hypothèses quant au support sur lequel elles ont été fixées. Peu à peu se dégage une image assez originale, bien différente de celle souvent implicite de personnages parés de nombreux bijoux «archaïques ». Face à l'abondance et à la diversité des éléments de parure recueillis à La Vergne, une telle approche ne pourra être que statistique.

Ces éléments de parure prennent un tout autre sens lorsqu'ils sont découverts en milieu sépulcral. Ils traduisent alors des intentions propres aux rituels de ces populations. Contribuant à la mise en scène des corps des défunts à l'occasion de leur inhumation, ils participent à rendre compte de l'univers symbolique qui entoure la vie et la mort des personnes concernées. La répartition de ces objets dans les tombes mésolithiques de La Vergne est très inégale. Les structures 1 et 2 correspondent respectivement à un fossé et à une fosse d'extraction de l'argile d'époque postérieure, 
où les éléments de parure recueillis sont de toute évidence en position remaniée. Ces structures ont largement recoupé trois ensembles funéraires dont seuls quelques lambeaux subsistent. Il s'agit des structures 3, 11 et 12. Aucun des ensembles d'objets de parure leur correspondant ne peut être considéré comme complet, même si la structure 3 a livré un mobilier très abondant.

21 La structure 10 en revanche n'est qu'à peine entamée par le creusement de la structure 2. On peut donc considérer que l'ensemble des objets de parure déposés dans la structure 10 est parvenu jusqu'à nous. Cela est également le cas pour la structure 7 , voisine. Les pièces provenant de chacune de ces tombes pourront être considérées séparément, au moins dans un premier temps. Les structures 7 et 10 ont livré près des deux tiers des objets de parure recueillis au cours de la fouille. À elle-seule, la structure 7 en compte près de la moitié. Si l'on y ajoute les éléments de parure provenant de la structure 3 on obtient un total de près de $95 \%$ des pièces étudiées. Chacun de ces trois ensembles se différencie très nettement par la nature des espèces représentées : $75 \%$ des nasses percées proviennent de la seule structure 7, alors que $60 \%$ des dentales proviennent en revanche de la structure 3. Ce dernier ensemble d'objets de parure se distingue également par la présence d'un petit lot de grands gastéropodes percés (comme les natices Euspira sp.). Quant à la structure 10, elle se caractérise par la présence de plusieurs lots de valves emboîtées les unes dans les autres.

\section{Un assemblage de signes}

La quatrième partie de cet article traite des coquilles telles qu'elles participent à la mise en scène de funérailles. Nous verrons alors que beaucoup ornaient en réalité autant d'objets distincts, enveloppant les dépouilles ou déposés à côté du défunt pour certains, tapissant le fond et les parois de la fosse pour d'autres. La parure corporelle proprement dite n'en constitue qu'une petite partie. Trop souvent l'étude de la parure préhistorique s'est limitée à celle de chaque élément pris séparément. Leur assemblage constitue un autre niveau d'analyse, tout aussi passionnant. Comme l'ensemble du costume, la parure affichée à la vue de tous, fait sens à ceux qui la regardent. Elle est chargée de valeurs esthétiques, symboliques ou culturelles, malheureusement difficiles à appréhender une dizaine de millénaires plus tard. C'était au départ pour rendre compte de la diversité des costumes traditionnels que le concept de culture matérielle a été énoncé, ensuite largement repris par les archéologues. La parure peut ainsi traduire l'appartenance ethnique, le statut, le sexe ou l'âge de la personne qui la porte. Son agencement rend compte de l'image que chaque individu se fait de son propre corps.

À La Vergne, le colmatage rapide des fosses, bien mis en évidence par les anthropologues sur le terrain, est un atout majeur; il aura au moins partiellement préservé la disposition initiale des éléments conservés de l'ornementation corporelle. Les traces d'usure, présentes autour de la perforation ou sur le test, attestent parfois du mode de suspension de la pièce. Chaque tombe a cependant livré le squelette de plusieurs individus inhumés simultanément ou successivement. De plus, la répartition spatiale des éléments de parure n'est pas homogène ; certains lots individualisés à la fouille peuvent correspondre à autant de parures, d'objets ou de pièces de vêtements différents. Ces données seront précieuses pour proposer une restitution de la parure associée à chaque défunt. La méthode de fouille employée à La Vergne, comprenant 
l'enregistrement systématique en plan et en 3 dimensions de chaque pièce, autorise une telle finesse d'analyse.

Tenter de lier par des interprétations cohérentes les observations effectuées sur la parure à ces différentes échelles d'analyse, de la tombe jusqu'à l'objet isolé, constitue l'une des ambitions de ce travail. Ce n'est qu'en fonction de ces résultats qu'il sera ensuite possible de tenter quelques comparaisons avec des ensembles homologues. Dans un premier temps, il conviendra d'étudier séparément pour chaque espèce les traces de façonnage et d'usure observées. L'état de conservation de ces objets de parure en coquille est en général assez bon. La nature du sol et du substrat calcaire n'y est sans doute pas étrangère. Il n'en reste pas moins que $60 \%$ des nasses présentent une surface altérée, pour ne prendre que ce seul exemple.

Dans la plupart des cas cependant, l'observation des traces de façonnage ou d'usure reste possible, sur au moins une partie de la coquille. La grille d'analyse retenue est différente suivant les espèces car seules deux d'entre elles sont présentes en nombre suffisant pour tenter une étude statistique des critères retenus. Parmi ces dernières, les dentales ont fait l'objet d'un façonnage sommaire, alors que les nasses présentent souvent une perforation d'origine anthropique. Les traces d'usure y sont également mieux marquées et plus diversifiées. L'étude des nasses est donc la plus détaillée. Elle comportera deux volets qui concernent respectivement la surface du test et la perforation. Les mêmes critères ont été appliqués, parfois nous l'avons dit de façon plus rapide, à l'étude des traces de façonnage ou d'usure présentes sur les autres espèces au niveau du test ou de la perforation.

\section{La recherche d'éléments de comparaison}

La cinquième et dernière partie de l'article discute de chacun des résultats précédemment exposés, qu'elle met en perspective à l'échelle de l'Europe occidentale, principalement pour la période du Mésolithique. Certains aspects seront abordés de façon plus synthétique, car ils ont déjà fait l'objet de quelques publications préalables au sein desquelles toutefois il n'était guère possible, en quelques pages, de détailler chaque élément d'un argumentaire que l'on trouvera ici exposé. D'autres, telle que la place des objets ornés dans la tombe, mériteront de plus larges développements, notamment du fait d'une bibliographie récente de plus en plus abondante sur le sujet. La conclusion s'étendra un peu sur la période suivante du Néolithique, comme par opposition, afin de mettre en exergue plusieurs formes d'actions des hommes ou des femmes sur la matière que l'étude de ce corpus illustre parfaitement.

\section{Les coquilles marines : détermination, description et lieu probable de collecte}

En préambule, la position géographique du site de La Vergne par rapport au littoral de l'époque est importante à préciser. Bien qu'il soit localisé à plus de 40 kilomètres du trait de côte actuel, et malgré un niveau de la mer plus bas au Mésolithique, il est probable que les influences marines étaient plus proches du site qu'elles ne le sont actuellement. Elles ont pu pénétrer dans les terres par le biais de la Paléocharente et de ses affluents comme La Boutonne dont différentes hypothèses quant à leur tracé ont été proposées par différents chercheurs (Hinschberger et Pauvret 1968, Barusseau 1973, 
Pinot 1973, 1998, Vigneaux et al. 1975). Lors de la mise en place des sépultures, entre 8600 et 8000 cal. BC, soit il y a environ 10500 à 10000 ans, le niveau de la mer oscillerait entre -26 et -18 mètres par rapport au niveau actuel (Stéphan et Gosselin 2014). Ces valeurs sont estimées à partir d'autres zones du littoral atlantique européen car elles ne sont pas documentées en l'état actuel dans la région pour la période concernée (Allard et al. 2008, Garcia-Artola et al. 2018). Utiliser cet intervalle de valeur est possible pour reconstituer la ligne de rivage approximative. Elle doit cependant prendre en compte certains phénomènes comme les mouvements tectoniques régionaux, la variation du taux de sédimentation différente de celle du taux de remontée eustatique du niveau marin, et la modification morphologique du trait de côte. C'est ce qui a été fait dans la région de la Charente, où André (1986, p. 26-28) a reconstitué une ligne de rivage approximative en considérant une différence de 11 mètres par rapport au niveau actuel à partir des données régionales pour la période de 10000 BP (fig. 9).

Fig. 9 - Localisation de La Vergne en fonction de trait de côte passé et actuel.

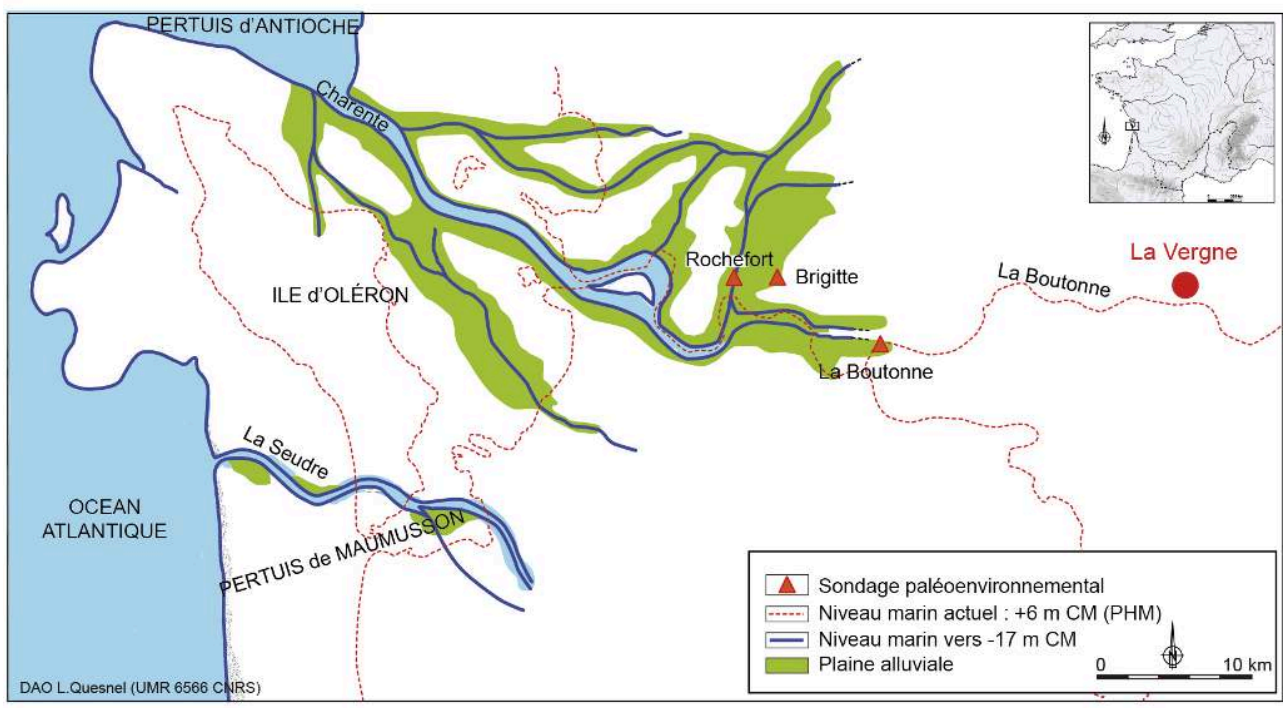

PHM : Plus Hautes Mers; $\mathbf{c m ~ : ~ n i v e a u ~ d e s ~ C a r t e s ~ M a r i n e s . ~}$

D’après André 1986 ; DAO L. Quesnel et C. Dupont.

Cette reconstitution tendrait donc à surestimer le niveau marin vis-à-vis des dernières synthèses publiées sur le sujet. D'après la reconstitution d'André (1986), la côte était distante du site de plus de $90 \mathrm{~km}$. Cependant, l'influence marine se faisait ressentir à l'intérieur des terres via le paléo- estuaire de la Charente. En effet, Carbonel et al. (1998), d'après le sondage Rochefort 102 (CPS102), notent une faune infralittorale de coquillages entre -12 et $-17 \mathrm{~m}$ NGF (soit un niveau marin de -11 à $-15 \mathrm{~m}$ ) pour une datation comprise entre 6000 et 8000 BP. Le lit du fleuve à Rochefort était donc entre -10 et $-15 \mathrm{~m}$ au-dessous du lit actuel. On peut imaginer qu'autour de 10000 BP la vallée de la Charente était beaucoup plus encaissée ce qui permettait la pénétration des eaux marines (marée de salinité) probablement à l'amont de Rochefort (Carbonel et al. 1998). Ainsi, les mollusques marins ont pu y pénétrer et pouvaient donc être accessibles sans doute à moins de $30 \mathrm{~km}$ du site. D'après différents sondages effectués à Rochefort 
(Regrain 1980, p. 83, Carbonel et al. 1998) l'existence d'anciens rivages a été mise en évidence, ce qui correspond à une importante influence marine. La marée dynamique devait se faire sentir jusque vers Saintes dans la Charente comme actuellement avant la construction d'un barrage à l'amont de Tonnay-Charente.

\section{Espèces déterminées}

La détermination des espèces a été effectuée avec l'aide d'ouvrages de biologie (Tebble 1966, Olivier 1975, Sabelli 1981, Philips 1987, Wye 1990, Poppe et Goto 1991, 1993, Dance 1993, Chavan 1996, Dautzenberg 1913). Une loupe binoculaire au grossissement 10×8 a été utilisée pour cette étape. L'identification des mollusques est basée sur la forme et l'épaisseur de la coquille, sa charnière pour les bivalves, son ornementation, ainsi que les empreintes laissées sur la coquille par la chair de l'animal, notamment les empreintes musculaire et ligamentaire. Les appellations scientifiques utilisées ou noms latins changent régulièrement. Celles utilisées dans le cadre de cette publication sont issues du World Register of Marine Species (WoRMS 2020) et ont été mises à jour depuis les premières publications de synthèse des parures en coquilles de La Vergne (Dupont 2006, Dupont et al. 2014, Laporte et Dupont 2019). Une liste de synonymes est proposée afin de pouvoir se reporter aux différentes appellations rencontrées dans les publications archéologiques (Taborin 1974, p. 112-123, 392-401, Barge 1982, p. 17-18, Newell et al. 1990, p. 104-106, Taborin 1993) et les ouvrages de biologie utilisés (Tebble 1966, Fretter et Graham 1976, 1978, 1981, 1985, Poppe et Goto 1991, 1993). Les noms vernaculaires sont aussi précisés.

$\mathrm{Au}$ total 18 espèces ou genres ont ainsi été déterminées : trois scaphopodes, 7 bivalves et 8 gastéropodes (tabl. 2 ; fig. 10). Des fiches complètes décrivant ces espèces et les critères qui ont permis de les déterminer ont d'ores et déjà été publiées (Dupont 2006). Parmi les mollusques marins, les patelles, spisules et moules n'ont été déterminées qu'au rang du genre. Cette limitation dans la détermination est liée pour les patelles à l'effacement partiel de leur ornementation et à la déformation de leur forme, pour les spisules et les moules à leurs cassures. Pour les dentales, au moins trois espèces en ont été identifiées (tabl.2). Elles l'ont été à partir du décompte des côtes dont elles sont ornées. Pour certains exemplaires à la surface usée, nous nous sommes limités à l'appellation Dentalium sp. du fait de l'effacement de leurs côtes. L'ensemble des dentales ont été réunis sous le genre Dentalium lors des bilans quantitatifs. Ce choix a été fait car les trois espèces concernées ont un biotope proche à la fois au niveau de leur position altitudinale et de leur substrat. 
Fig. 10 - Les mollusques marins déterminés à La Vergne.

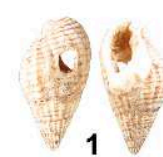

1

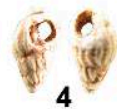

2
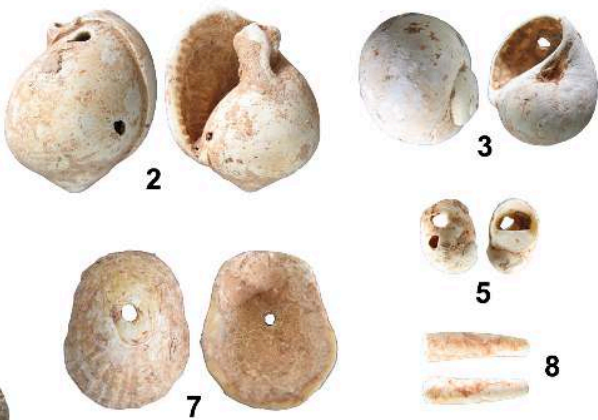

5

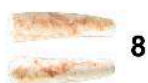

6
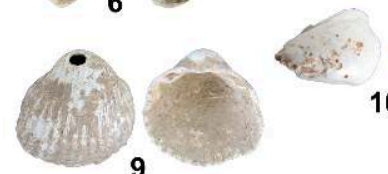

10

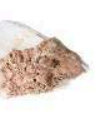

11

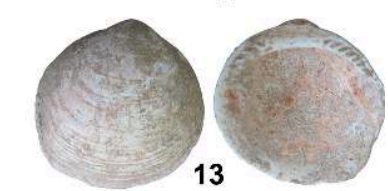

12
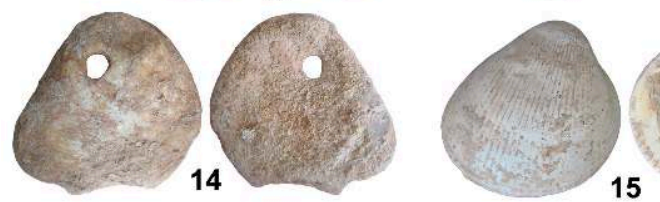

15

1. Tritia reticulata $(L=22 \mathrm{~mm})$; 2. Semicassis saburon $(L=53 \mathrm{~mm})$; 3. Euspira catena $(L=25 \mathrm{~mm})$;

4. Tritia incrassata $(L=9 \mathrm{~mm})$; 5. Theodoxus fluviatilis $(L=8 \mathrm{~mm}) ; \mathbf{6}$. Ocenebra erinaceus $(L=19 \mathrm{~mm})$;

7. Patella sp. $(L=41 \mathrm{~mm})$; 8. Dentalium sp. $(L=12 \mathrm{~mm})$; 9. Cerastoderma edule $(L=29 \mathrm{~mm})$;

10. Mytilus sp. $(L=20 \mathrm{~mm})$; 11. Spisula sp. $(L=33 \mathrm{~mm})$; 12. Venus verrucosa $(L=36 \mathrm{~mm})$;

13. Glycymeris glycymeris $(L=51 \mathrm{~mm}) ;$ 14. Ostrea edulis $(L=70 \mathrm{~mm}) ; \mathbf{1 5}$. Laevicardium crassum $(\mathrm{L}=54 \mathrm{~mm})$.

Photos et DAO C. Dupont. 
Tabl. 2 - Liste des genres et espèces déterminés à La Vergne avec leur appellation scientifique valide et leurs synonymes rencontrés dans les publications archéologiques.

\begin{tabular}{|c|c|c|}
\hline \multirow[b]{2}{*}{ Nom vernaculaire } & \multicolumn{2}{|c|}{ Appellations scientifiques } \\
\hline & $\begin{array}{l}\text { Autres appellations rencontrées dans les } \\
\text { ouvrages archéologiques }\end{array}$ & $\begin{array}{l}\text { Appellation valide au 09/04/2020 } \\
\text { (WoRMS 2020) }\end{array}$ \\
\hline \multicolumn{3}{|l|}{ Classe des scaphopodes } \\
\hline \multirow{4}{*}{ Le dentale } & Dentalium sp. & Dentalium Linnaeus, 1758 \\
\hline & Dentalium entalis, & Antalis entalis (Linnaeus, 1758) \\
\hline & Dentalium dentalis & Antalis dentalis (Linnaeus, 1758) \\
\hline & Dentalium vulgare, D. tarentinum, D. striolatum & Antalis vulgaris (da Costa, 1778) \\
\hline \multicolumn{3}{|l|}{ Classe des gastéropodes } \\
\hline \multirow[b]{2}{*}{ La nasse réticulée } & $\begin{array}{l}\text { Hinia reticulata, Nassa. nitdia, H. nitida, N. neri- } \\
\text { tea, forme nida, Nassarius nitidus, Nassa mamil- } \\
\text { lata, Nassarius reticulatus }\end{array}$ & Tritia nitida (Jeffreys, 1867) \\
\hline & $\begin{array}{l}\text { Hinia reticulata, Nassa neritea, } N \text {. reticulata,N. } \\
\text { reticulatus, Arcularia reticulata, Buccinum retic- } \\
\text { ulatum, Hinia reticulata, Hinia servaini, Nassa } \\
\text { mamillata. }\end{array}$ & Tritia reticulata (Linnaeus, 1758) \\
\hline La nasse & $\begin{array}{l}\text { Hinia incrassata, Buccinum undatum, Nassa in- } \\
\text { crassata, Nassarius coralligenus, N. incrassatus, } \\
\text { N. lacepedei }\end{array}$ & Tritia incrassata (Strøm, 1768) \\
\hline La natice & $\begin{array}{l}\text { Natica catena, Natica sp, } N \text {. alderi var nitida, } \\
\text { Cochlea catena, Lunatia catena, Lunatia moni- } \\
\text { lofera. }\end{array}$ & Euspira catena (da Costa, 1778) \\
\hline La nérite des rivières & $\begin{array}{l}\text { Neritina fluviatilis, Nenita fluviatilis, N. littoralis, } \\
\text { Theodoxus Iutetianus, T. trifasciatus, Neritina flu- } \\
\text { viatilis }\end{array}$ & Theodoxus fluviatilis (Linnaeus, 1758) \\
\hline Le murex & $\begin{array}{l}\text { Ocenebra erinacea, Cerastostoma erinaceum, } \\
\text { Murex erinaceus, Tritonalia erinaceum }\end{array}$ & Ocenebra erinaceus (Linnaeus, 1758) \\
\hline La patelle & Patella sp. & Patella Linnaeus, 1758 \\
\hline Le casque & Cassis saburon, Phalium saburon & Semicassis saburon (Bruguière, 1792) \\
\hline \multicolumn{3}{|l|}{ Classe des bivalves } \\
\hline La bucarde de Norvège & Cardium norvegicum & Laevicardium crassum (Gmelin, 1791) \\
\hline L'amande de mer & Glycymeris sp.,Pectunculus glycymeris & Glycymeris glycymeris (Linnaeus, 1758) \\
\hline La moule & Mytilus sp. & Mytilus Linnaeus, 1758 \\
\hline La spisule & Spisula sp. & Spisula Gray, 1837 \\
\hline L'huitre plate & Ostrea sp., O. cristata, O. adriatica & Ostrea edulis Linneus, 1758 \\
\hline La praire & - & Venus verrucosa Linnaeus, 1758 \\
\hline La coque & Cardium edule & Cerastoderma edule (Linnaeus, 1758) \\
\hline
\end{tabular}

31 Le cas de la nasse réticulée est plus complexe. Lors des premières analyses des coquilles de La Vergne cette espèce possédait une variété nitidus (Dupont 1998), elle était alors appelée Nassarius reticulatus var. nitidus. À la date de la rédaction du présent article, cette variété est reconnue comme une espèce, Tritia nitida, qui se différencie de Tritia reticulata (WoRMS, 2020). L'ensemble des analyses et publications réalisées ont été faites sur la base de la présence de la nasse réticulée Tritia reticulata. Dans les bilans quantitatifs et les parties suivantes de cet article nous avons choisi de garder cette appellation. Lors de l'étude nous avions cependant été marqués par l'abondance de nasses réticulées se rapprochant de la forme de Tritia nitida (fig. 11). 
Fig. 11 - Échantillon de nasses de La Vergne.

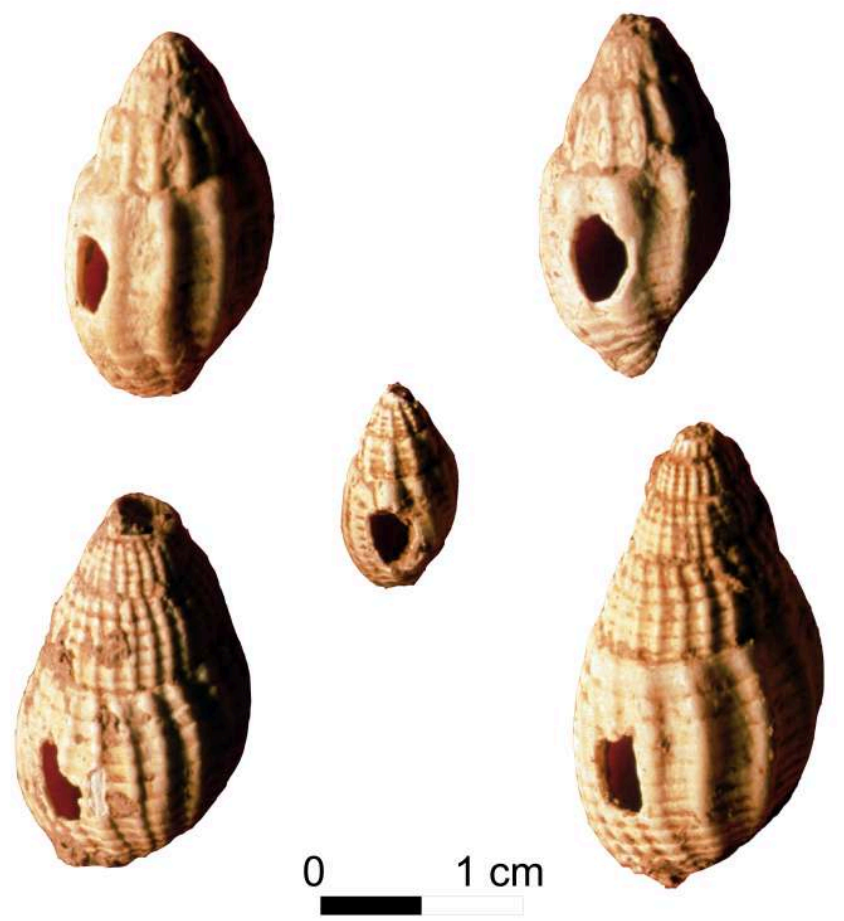

Photos et DAO C. Dupont.

D'autre part, le milieu de vie de T. nitida est plus confiné. Une étude de référentiels actuels a ensuite été réalisée afin de confirmer cette impression visuelle de présence de T. nitida au sein de l'échantillon de La Vergne par des critères biométriques (Dupont et Gruet 2000). Dans ce but, différents lots de nasses ont été étudiés en fonction du degré d'exposition aux houles de leur milieu : de côtes battues à des côtes plus abritées. Une telle démarche avait déjà été réalisée et s'était avérée prometteuse pour d'autres espèces comme le pourpre Nucella lapillus (Crothers 1973, Moore 1985, Fischer 1995, p. 279) et la patelle Patella sp. (Fischer 1995, p. 279). Parmi les critères retenus, le rapport du nombre de côtes décomptées pour le dernier tour de spire sur la largeur de la coquille de la nasse montre que Tritia reticulata a un nombre relativement plus important de côtes que T. nitida (fig. 12). 
Fig. 12 - Référentiels actuels de nasses réticulées.

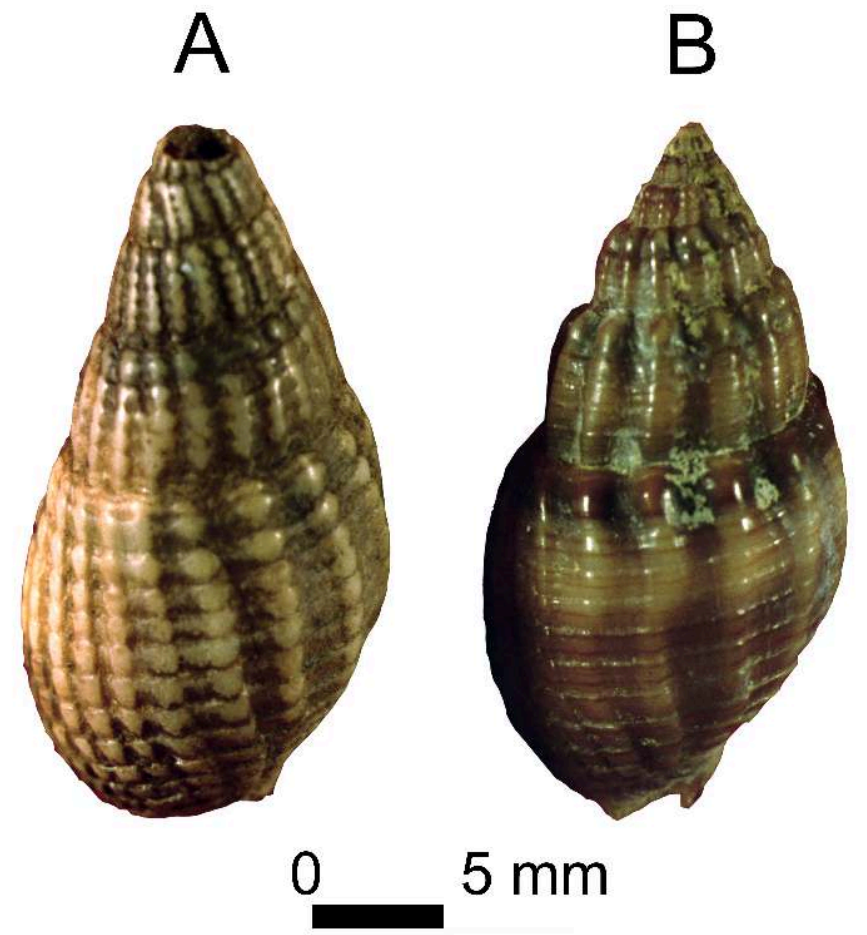

A. Tritia reticulata; B. Tritia nitida.

Photos et DAO C. Dupont.

33 L'application de ce critère sur une trentaine d'exemplaires de nasses de La Vergne montre que la variété de l'échantillon archéologique se rapproche du milieu abrité et tend à confirmer la présence de l'espèce T. nitida (fig. 13). 
Fig. 13 - Variations des formes de nasses réticulées en fonction du mode d'exposition de la côte.

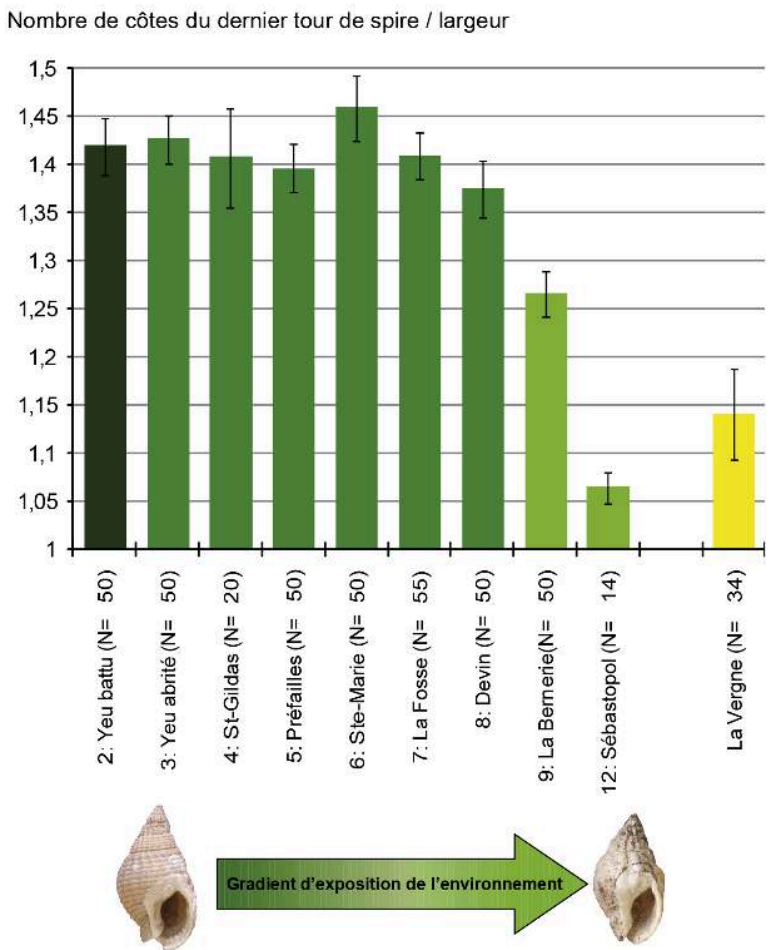

D’après Dupont et Gruet 2000.

Avec 18 espèces représentées, la richesse spécifique représentée est importante pour un site éloigné de plusieurs dizaines de kilomètres du trait de côte lors de son fonctionnement. Ces espèces ont ensuite été quantifiées pour connaitre leurs proportions au sein des milliers de coquilles analysées.

\section{Spectre malacofauniques : de la richesse à la proportion des espèces}

Le spectre malacofaunique montre que, malgré une richesse spécifique supérieure à dix, deux espèces que sont les nasses réticulées et les dentales représentent à elles seules $97 \%$ de la malacofaune du site (tabl. 3 ; fig. 14). Cette composition binaire de la malacofaune a souvent été observée et paraît être un caractère constant de la parure mésolithique du littoral atlantique français (Taborin 1974, p. 158, 164, 395, May 1986, p. 119). Parmi ces deux espèces, Tritia reticulata domine largement avec plus de $66 \%$ des restes de la malacofaune totale. Cette espèce reste majoritaire en prenant en compte le poids des coquilles avec $48 \%$ de la masse des coquilles analysées à La Vergne. Cependant, les dentales (espèce de petite taille) voient leur abondance relative diminuer au profit de grands bivalves (tabl. 3). 
Tabl. 3 - Bilan quantitatif de la malacofaune de l'ensemble des coquilles découvertes à La Vergne.

\begin{tabular}{|l|l|c|c|c|c|c|c|}
\hline Nom scientifique & Nom vernaculaire & Masse (g) & \% Masse & NR & \% NR & NMI & $\%$ NMI \\
\hline Patella sp. & La patelle & 69,56 & 3,5 & 11 & 0,3 & 11 & 0,3 \\
\hline Tritia incrassata & La nasse & 26,69 & 1,4 & 34 & 1,0 & 34 & 1,0 \\
\hline Ocenebra erinaceus & Le murex & 0,98 & 0,0 & 1 & 0,0 & 1 & 0,0 \\
\hline Ostrea edulis & L'huître plate & 27,49 & 1,4 & 1 & 0,0 & 1 & 0,0 \\
\hline Mytilus edulis & La moule & 1,13 & 0,1 & 2 & 0,1 & 2 & 0,1 \\
\hline Tritia reticulata \& T. nitida & La nasse réticulée & 949,10 & 48,1 & 2194 & 66,5 & 2194 & 66,5 \\
\hline Spisula sp. & La spisule & 6,55 & 0,3 & 2 & 0,1 & 2 & 0,1 \\
\hline Venus verrucosa & La praire & 5,83 & 0,3 & 1 & 0,0 & 1 & 0,0 \\
\hline Glycymeris glycymeris & L'amande de mer & 105,84 & 5,4 & 5 & 0,2 & 5 & 0,2 \\
\hline Laevicardium crassum & La bucarde de Norvège & 430,03 & 21,8 & 14 & 0,4 & 14 & 0,4 \\
\hline $\begin{array}{l}\text { Dentalium sp. (Antalis enta- } \\
\text { lis, } \text { A. dentalis, } \text { A. vulgare) }\end{array}$ & Le dentale & 93,82 & 4,8 & 1012 & 30,7 & 1012 & 30,7 \\
\hline Cerastoderma edule & La coque & 3,89 & 0,2 & 1 & 0,0 & 1 & 0,0 \\
\hline Euspira catena & La natice & 111,84 & 5,7 & 15 & 0,5 & 15 & 0,5 \\
\hline Semicassis saburon & Le casque & 141,25 & 7,2 & 2 & 0,1 & 2 & 0,1 \\
\hline Theodoxus fluviatilis & La nérite des rivières & 0,19 & 0,0 & 2 & 0,1 & 2 & 0,1 \\
\hline Total & $\mathbf{1 9 7 4 , 1 9}$ & & $\mathbf{3 2 9 7}$ & & $\mathbf{3 2 9 7}$ & \\
\hline
\end{tabular}

NR : Nombre de restes; NMI : Nombre Minimum d'Individus.

Fig. 14 - Spectre malacofaunique de La Vergne en fonction du nombre de restes NR.

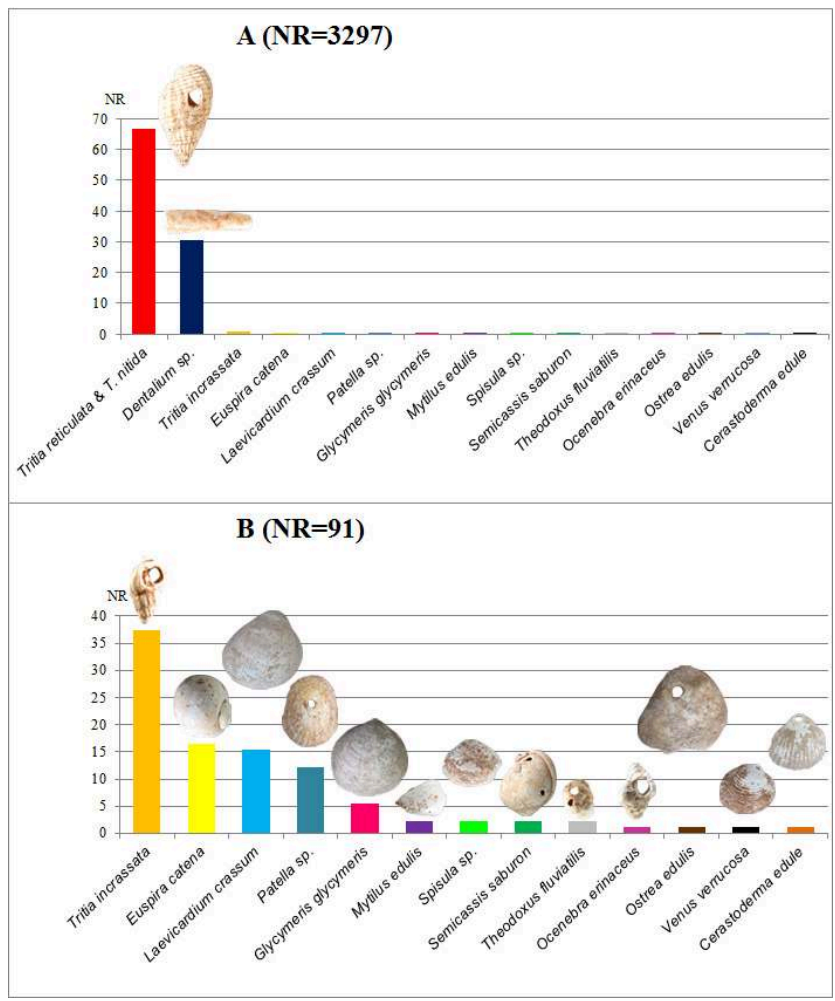

A. Sur l'ensemble des coquilles; B. Exclusion faite des deux espèces majoritaires.

Photos et DAO C. Dupont.

La seconde espèce majoritaire, en prenant la masse en compte, est alors la bucarde de Norvège représentée par 14 valves. Ainsi, les espèces importantes en taille comme Laevicardium crassum, Semicassis saburon et Glycymeris glycymeris voient leurs proportions augmenter. Une tendance générale s'observe à La Vergne : les grands coquillages peu 
nombreux en NR sont plus visibles si leur poids est pris en compte à l'inverse des petites espèces qui voient leur proportion diminuer. L'encombrement des coquilles par le poids et le volume de celles-ci ont pu agir sur le choix des coquilles. Il ne faut donc pas négliger les grandes espèces en faible nombre pour la caractérisation culturelle d'un groupe par la malacofaune, car certaines coquilles de grande taille ont pu être volontairement choisies même si leur faible nombre tendrait à les négliger. Parmi les espèces représentées, huit parmi les quinze sont présentes à un ou deux exemplaires (tabl. 3). Plusieurs hypothèses à ce stade de l'étude peuvent être émises concernant le nombre de restes très hétérogène des espèces.

L'accessibilité de la ressource et des choix guidés par une appartenance à un groupe culturel peuvent agir sur les quantités relatives de chaque espèce (Fischer 1995 p. 284). Dans le cas d'une accessibilité liée à la biologie du coquillage, la liste des espèces présentes peut rendre compte de la faune locale, de la proximité et de l'éloignement de la mer (Taborin 1974 p.155). La rareté des espèces présentes uniquement par quelques exemplaires peut s'expliquer par l'absence à proximité du site de leurs biotopes. Elles ont pu être retrouvées échouées. Ceci peut être le cas par exemple de Glycymeris glycymeris et surtout de Semicassis saburon dont les biotopes seront exposés par la suite.

l y a également pu avoir une dégradation post-dépositionnnelle des coquilles les plus fragiles (Dyke 1996 p. 134). La présence de la moule Mytilus edulis par uniquement deux débris peut appuyer cette hypothèse, d'autant plus que la fragilité de cette espèce est régulièrement soulignée en archéo-malacologie (Dupont 2006, Mougne 2015). Ce bivalve possède en effet, une coquille mince et très fragile qui résiste mal au poids du sédiment. C'est peut-être également le cas pour Theodoxus fluviatilis qui possède un test peu épais. Mais l'hypothèse, d'une relation entre la fragilité des coquilles et leur faible représentativité dans le spectre de La Vergne tend à être écartée pour d'autres espèces qui sont de très grande taille et d'épaisseur importante. C'est le cas de l'huître Ostrea edulis et de Semicassis saburon.

Une autre hypothèse peut être avancée. Des choix notamment d'ordres culturels ont aussi pu aboutir à ne retenir qu'un petit nombre d'espèces parmi celles disponibles.

À ce stade de la réflexion, deux hypothèses peuvent ainsi expliquer le choix des espèces et leurs proportions à La Vergne : celle de l'accès différencié de la ressource en fonction des espèces dans les lieux de collecte et celle de choix d'ordre culturel. Afin de tester la première hypothèse, une étude détaillée du biotope de chaque espèce a été réalisée.

\section{Des biotopes aux lieux de collecte}

\section{Biogéographie et accessibilité des espèces sur le littoral charentais}

41 Dans un premier temps, les distributions biogéographiques des mollusques déterminés ont été étudiées afin de savoir si les espèces représentées à La Vergne étaient cohérentes avec celles actuellement présentes sur le littoral charentais. Rappelons que la répartition de la malacofaune est liée à de nombreux facteurs écologiques et que celui de la température est déterminant (Dyke 1996, p. 129, Bailey et Parkington 1988). Des provinces biogéographiques actuelles sont définies en fonction de la température des océans: zone arctique, tempérée froide, tempérée chaude, tropicale (Babin et Glémarec 1971). Les variations de température qu'ont subies les mers sont corrélées à 
des changements des conditions océanographiques. Ainsi la modification du parcours de certains courants comme la Gulf-Stream, liée à l'avancée ou au recul des inlandsis, a fait varier la température de l'eau et donc l'emplacement des provinces biogéographiques. La méthode utilisée ici consiste à préciser la répartition biogéographique des espèces actuelles et à la modifier en fonction de leur présence dans le site mésolithique, afin d'observer d'éventuels changements climatiques qui ont eu lieu.

Seul le cas de Semicassis saburon a attiré notre attention. Sa présence au Mésolithique le long du littoral charentais le situe un peu plus au nord de sa répartition actuelle. En effet, elle est présente sur la côte basque dans le golfe de Gascogne (trouvée vivante par confirmation écrite de M. Borja à Y. Gruet). Le Semicassis saburon étant une espèce méridionale sa présence au Mésolithique plus au nord qu'actuellement indiquerait un climat légèrement plus chaud à l'époque du site qu'actuellement. En ce qui concerne les autres espèces, le réchauffement du climat, c'est-à-dire leur migration vers le nord, n'en exclut aucune. Le site étudié est daté de 8500-8000 cal. BC (Duday et Courtaud 1998). Ceci correspond à une phase de réchauffement général du climat commencée il y a plus de 10000 ans. La température océanique pouvait donc être de quelques degrés supérieurs à celle qui existe actuellement (Kaufman et al. 2020, Martin et al. 2020). Cette démarche s'appuie sur une seule espèce, Semicassis saburon, qui est la seule à pouvoir nous donner des informations sur de légères variations climatiques les autres espèces étant nordiques. Elle a très bien pu vivre et être recueillie à proximité de lieux confinés qu'offre l'estuaire de la Charente. On sait d'ailleurs que l'espèce y vivait il y a 8000 ans d'après les résultats d'un sondage réalisé juste à l'amont de Rochefort (Carbonel et al. 1998). D'après leurs distributions biogéographiques et les hypothèses publiées sur l'évolution du climat, l'ensemble des espèces déterminées à La Vergne ont pu être trouvées sur le littoral atlantique au Mésolithique. Nous avons ainsi privilégié cette hypothèse dans ce qui suit.

\section{À la recherche des lieux de collecte potentiels}

La détermination des espèces donne également accès à plusieurs paramètres du biotope des mollusques marins: le degré de salinité, le taux d'oxygène, la température, le substrat, la profondeur (Babin et Glémarec 1971, Shackleton 1988)...

La majorité des espèces de La Vergne sont marines à l'exception de Theodoxus fluviatilis. Cette espèce vit en effet en eau douce, mais aussi, en eau saumâtre. Elle peut supporter de faibles influences d'eaux marines avec une salinité qui ne dépasse pas les $18 \%$ (Hubendick 1947, Jaeckel 1952). La prépondérance de coquilles strictement marines est un fait régulier dans la parure (Rozoy 1978 p. 1124, Taborin 1982, p. 43, Binant 1991-2, p. 92). Ceci s'explique en partie par le fait que les coquilles marines possèdent des tests plus épais et sont plus solides que ceux de la malacofaune vivant en eaux douces (Binant 1991-2, p. 92, Barge-Mahieu et Taborin 1991, p. 1). L'utilisation des coquilles en tant que parure nécessite des tests suffisamment solides pour subir à la fois le percement et le port (traction du lien, entrechoquement des coquilles sur un même objet). La présence d'espèces marines est en relation avec la relative proximité de la mer par rapport au site. En effet, comme nous l'avons vu en introduction, le sondage de Rochefort présentait lors de datations contemporaines de La Vergne une côte rocheuse soumise aux influences marines, ce qui fait que ces coquilles étaient accessibles à moins de 30 kilomètres. Theodoxus fluviatilis a, quant à elle, été recueillie en eaux douces, voire 
en eaux saumâtres ce qui a permis son ramassage potentiellement sur les rives de la Boutonne. Ces éléments ajoutés à une marée dynamique qui pouvait être ressentie audelà de Saintes pour la Charente et dans le lit de La Boutonne offrent des possibilités de déplacements en bateau par les hommes et les femmes du Mésolithique.

L'analyse des substrats des différentes espèces déterminées va permettre de savoir où les mollusques marins ont pu être sélectionnés le long de l'axe Charente, soit en sa partie avale là où les rochers pouvaient dominer, soit en partie amont, plus abritée (fig. 15).

Fig. 15 - Bilan des substrats et niveaux altitudinaux des espèces de La Vergne.

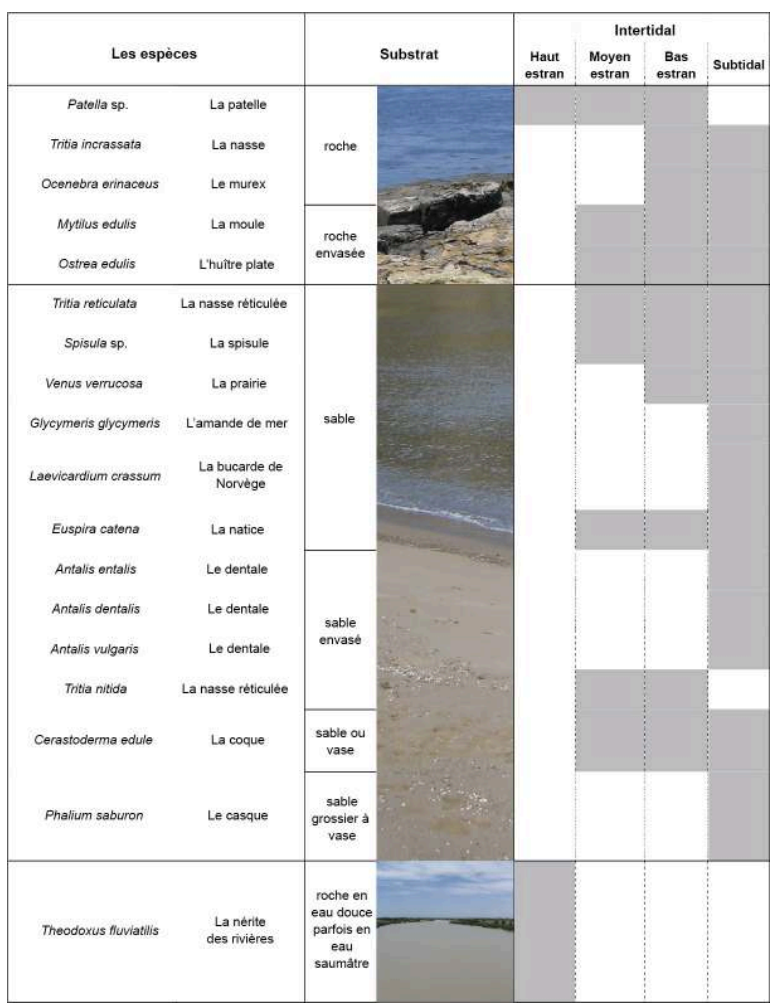

DAO C. Dupont.

Le cumul des proportions des espèces en fonction de leurs nombres de restes montre que plus de $98 \%$ sont inféodées à un substrat meuble dont plus de $97 \%$ à un sédiment sableux ayant pu subir un envasement (fig. 16). 
Fig. 16 - Proportions des substrats et niveaux altitudinaux des mollusques marins de La Vergne en fonction de leur nombre de restes.
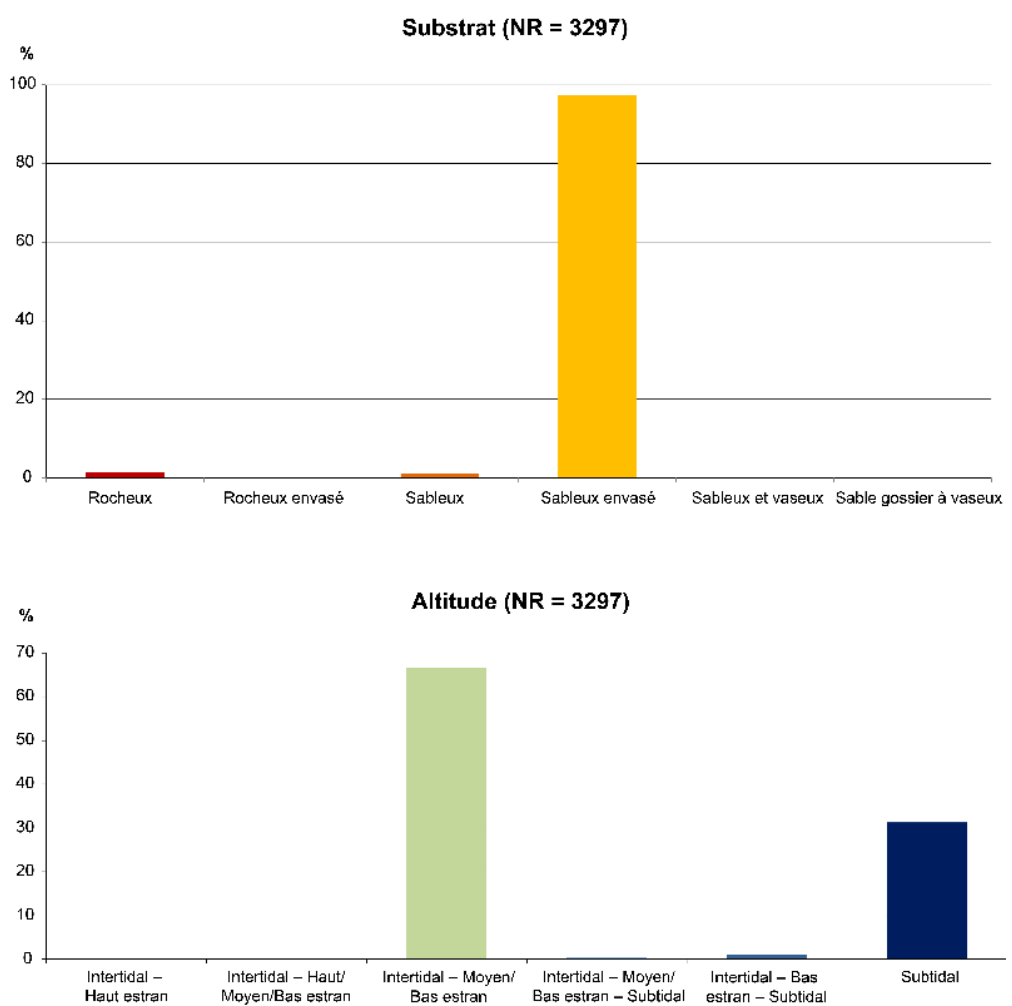

DAO C. Dupont.

Le substrat majoritairement représenté à La Vergne est en accord avec une collecte dans un milieu abrité comme pouvait l'être le paléoestuaire de La Charente. La présence de Tritia nitida corrobore ce caractère abrité de la zone de collecte. Même si l'hypothèse d'un échange avec d'autres groupes humains ne peut être exclue, les coquilles utilisées à La Vergne ont aussi pu être ramassées par la population qui a fréquenté le site lors d'un ou de plusieurs déplacements. L'analyse isotopique des squelettes humains montre que les personnes enterrées à La Vergne n'ont pas consommé de ressources marines (Schulting et al. 2008), en tous les cas pas assez pour marquer leurs ossements du signal marin. Ce résultat reste en accord avec ce que nous connaissons de la consommation des fruits de mer par les chasseurs-cueilleurs du littoral atlantique français. En effet, celle-ci semble cantonnée à moins d'un kilomètre du littoral (Dupont 2006).

\section{État de la matière première lors de son ramassage}

\section{Du niveau altitudinal de vie du mollusque à son état lors de sa collecte}

Les mollusques marins peuvent être intertidaux ou subtidaux. Ceux qui sont intertidaux sont inféodés à l'estran, c'est-à-dire la zone qui se découvre à chaque marée basse; tandis que les espèces subtidales sont celles qui sont constamment immergées. La zone intertidale peut être décomposée en plusieurs niveaux de l'estran, calqués sur la composition algale des littoraux actuels (Bernier et Gruet 2011). Les différents niveaux altitudinaux des espèces déterminées à La Vergne ont été résumés (fig. 15). Ils montrent dans un premier temps que plusieurs espèces sont strictement subtidales: 
l'amande de mer, la bucarde de Norvège, les dentales et le casque. Rapportées aux nombres de restes, ces espèces non accessibles à pied sec à marée basse représentent $31 \%$ de l'assemblage de La Vergne (fig. 16). Ce résultat va à l'encontre de ce qui est connu du comportement des populations mésolithiques du littoral atlantique français lors de leur quête de nourriture. En effet, ces populations semblent connaitre les coefficients de marée et ne mettent pas les pieds dans l'eau pour se nourrir (Dupont 2006). L'ensemble des espèces attestées comme ayant été consommées dans la zone d'étude est accessible dès les niveaux haut à moyen de l'estran. Ainsi, les coquilles utilisées en parure à La Vergne ont pu être sélectionnées à ce stade de l'analyse suivant deux procédés, soit elles ont été ramassées vivantes en pratiquant des techniques telles l'apnée ou le dragage, soit elles ont été collectées mortes échouées sur la place. Certaines espèces comme le Semicassis saburon qui vivent à plusieurs mètres ou dizaines de mètres de profondeur nous encouragent à privilégier la seconde hypothèse. De nombreux stigmates viennent la corroborer sur le matériel de La Vergne. À la mort de l'animal, les coquilles subissent le ressac de la mer. Les grains de sable vont venir percuter les tests. L'intégralité de la surface de la coquille va être touchée, même si les stigmates seront plus lisibles dans les zones les plus proéminentes ou convexes et décrits comme une abrasion différentielle (Rolin 1971, p. 78, Barge 1982, p. 63). Sur les bivalves, le crochet est souvent attaqué (fig. 17).

Fig. 17 - Exemples de stigmates liés à l'usure marine sur les bivalves de La Vergne (A1 et B1) comparés à des référentiels ramassés vivant (A2 et B2).

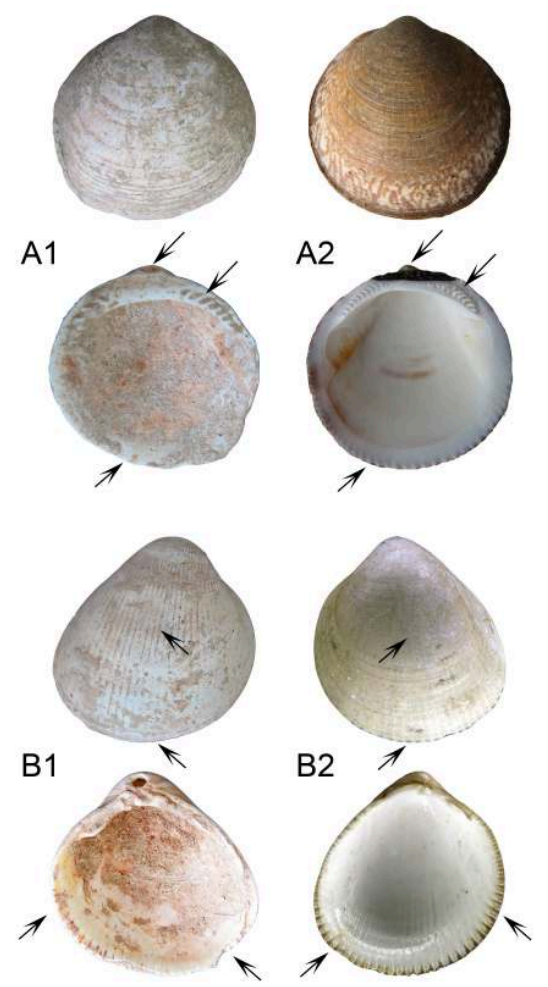

A1. Amande de mer $(L=51 \mathrm{~mm})$. Les flèches montrent une réduction et un percement de son crochet, des cassures et régularisation de son bord coquillier avec effacement des reliefs sur la face interne, et une réduction du relief de ses dents par rapport à un individu $A 2$ ramassé vivant $(L=48 \mathrm{~mm})$; B1. Bucarde de Norvège $(L=54 \mathrm{~mm})$. Les flèches montrent une réduction et un percement de son crochet, des cassures et régularisation de son bord coquillier avec effacement des reliefs sur la face interne par rapport à un individu B2 ramassé vivant $(\mathrm{L}=38 \mathrm{~mm})$.

Photo B2 : P. Lozouet ; DAO C. Dupont. 
Une usure marine prolongée peut même aller jusqu'à un percement de celui-ci. Nous verrons dans ce qui suit qu'il est probable que certaines des perforations utilisées pour fixer les coquilles soient en effet d'origine naturelle. De même, le relief des dents de la charnière est réduit. Ce phénomène $\mathrm{a}$, par exemple, été observé sur les amandes de mer de La Vergne (fig. 17). L'entrechoquement des coquilles dans les flux et reflux marins peut provoquer des cassures du bord des valves. L'altération suit les arrêts de croissance. La surface des valves est également atteinte et met au jour la structure de la coquille. Ce phénomène est visible sur la surface externe de l'amande de mer en soulignant les lignes concentriques et de la bucarde de Norvège en mettant à nu ses ornementations radiales (fig. 17). De même, sur les gastéropodes, l'usure marine attaque les zones convexes peu à peu par frottement des tests sur le sable. Le dernier tour de spire qui est le plus grand est souvent le premier atteint (Barge-Mahieu et Taborin 1991, p. 13) avec l'apex. Cette usure peut même aboutir au percement sur la partie dorsale du dernier tour de spire (fig. 18B2). Un murex de La Vergne possède nombres des stigmates liés à une érosion marine (fig. 18C1). Son apex après avoir été cassé le long d'un des tours de spire a été arrondi. L'ensemble des côtes qui ornent sa coquille ont été en partie effacées. La partie appointée que représente le canal siphonal aussi été réduite et arrondie. Certains apex de patelles présentent, quant à eux, une desquamation progressive (fig. 18). Sur les Semicassis saburon un des individus est particulièrement usé. Cela se traduit par une disparition de ces côtes d'ornementation qui se distinguent uniquement au niveau de son labre. De même, plusieurs de ces stigmates ont été observés sur les nasses réticulées. Certains sont des épifaunes qui percent parfois l'intégralité du test et qui indiquent la mort de l'animal avant le ramassage de la coquille sur la plage (fig. 18B4). D’autres sont liés à l'érosion marine et affectent l'intégralité du test. L'important nombre de nasse a amené à leur appliquer une typologie en fonction de l'état de conservation des coquilles. Pour mettre en place cette typologie, deux lots actuels de nasses rejetés sur la plage ont été utilisés. Ils proviennent de deux plages différentes de la côte atlantique : celle de Pénestin près de l'estuaire de la Vilaine en lot $n^{\circ} 1$ et celle du Polder de Sébastopol sur l'île de Noirmoutier en lot $n^{\circ} 2$. Une typologie de dégradation des coquilles par la mer a été réalisée à partir de ces échantillons (fig. 19). 
Fig. 18 - Exemples de stigmates liés à l'usure marine sur les gastéropodes de La Vergne (A1, B1, $B 4$ et $C 1)$ comparés à des référentiels ramassés vivant $(A 2, B 3, C 3)$ et morts $(B 2)$.

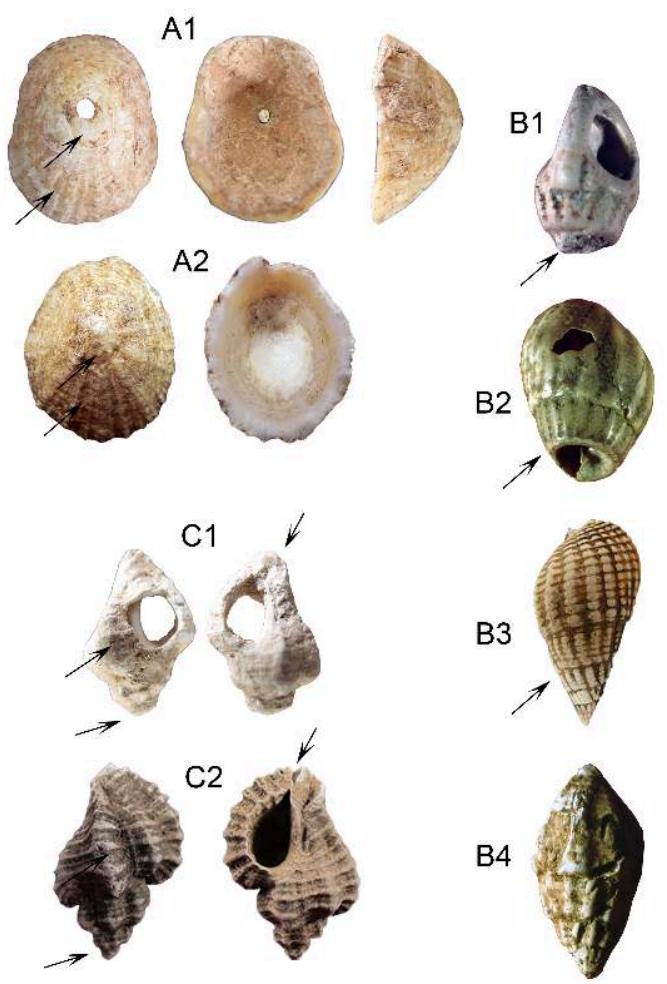

A1. Patelle $(L=41 \mathrm{~mm})$ qui montre une desquamation de son apex et un effacement de ses côtes par rapport à une patelle de Beg-er-Vil $A 2$ ramassée vivante $(L=34 \mathrm{~mm})$; B1. Nasse réticulée montrant un poli de l'ensemble de sa surface et présentant un poli des sutures de spires cassées $(L=10 \mathrm{~mm})$;

B2. Nasse percée par érosion marine $(L=12 \mathrm{~mm})$; B3. Nasse collectée vivante $(L=20 \mathrm{~mm})$;

B4. Nasse avec perforations de vers marins Polydora sp. ; c1. Murex présentant de nombreux stigmates liés à l'érosion marine (cassure et poli de l'apex, poli du canal siphonal, réduction du relief des côtes et perforation, $L=19 \mathrm{~mm})$; c2. Murex de Beg-er-Vil ramassé vivant $(L=30 \mathrm{~mm})$.

Photos et DAO C. Dupont. 
Fig. 19 - Typologie de dégradation des nasses réticulées basée sur l'observation de référentiels actuels échoués morts.

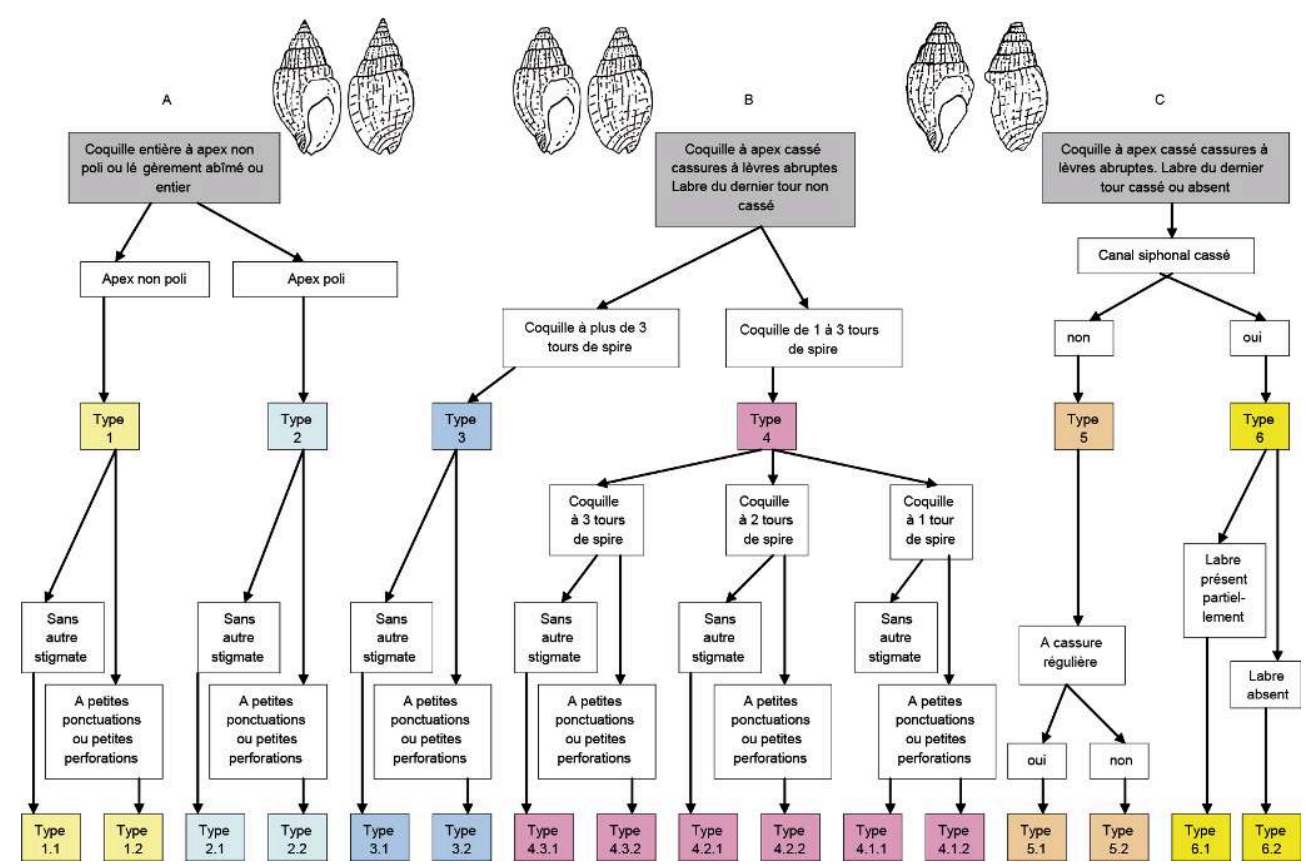

Dessin Y. Gruet; DAO C. Dupont.

Plusieurs types ont été définis de 1.1 à 6.2 : c'est-à-dire du moins dégradé au plus détérioré. L'ensemble de ces types peut être regroupé en trois catégories principales. La catégorie $\mathrm{A}$ représente des coquilles à apex entier, la $\mathrm{B}$ à celles à apex cassés. La catégorie $\mathrm{C}$ a un stade minimum de dégradation caractérisé par la cassure à la fois de l'apex et du péristome. Cette dernière catégorie va jusqu'à la dégradation totale des coquilles. Appliquée aux deux lots de référentiels actuels cette typologie montre des degrés d'usure différente des nasses suivant le mode d'exposition aux houles des plages. Le lot $n^{\circ} 2$ en milieu plus confiné est le moins dégradé. Il est représenté en majorité par la catégorie A et par un peu d'exemplaires en catégorie $\mathrm{B}$. Le lot $\mathrm{n}^{\circ} 1$ sur une page plus exposée possède la majorité de ses apex cassés. Les trois catégories sont représentées. Cette typologie a ensuite été appliquée aux coquilles de nasses réticulées de La Vergne en faisant abstraction de la perforation. La majorité des coquilles de La Vergne est de type B, à savoir à apex cassé et à péristome entier (fig. 20). Ce profil le rapproche du référentiel actuel 1 de plage exposée à la différence près que le lot archéologique a une plus faible proportion de coquilles en catégorie $\mathrm{A}$ au profit de la catégorie $\mathrm{C}$ par rapport aux référentiels actuels. Ainsi, l'état de dégradation général des coquilles archéologique est plus dégradé que les échantillons de référence (fig. 20). Cela peut s'expliquer par le port de la parure qui a pu amener par entrechoquement la cassure des apex. Certains fouilleurs nous ont aussi informés que lors de la fouille la dureté du sédiment face à la fragilité des coquilles a parfois provoqué la détérioration des apex. Ces cassures récentes sont souvent identifiables car elles correspondent à des cassures fraiches exemptes de sédiment. La détérioration accentuée des nasses de La Vergne peut ainsi s'expliquer par l'action de la mer, par le port des coquilles qui a pu entres autres fragiliser le labre du fait de la présence de la perforation, par des processus taphonomiques mais aussi par les fouilleurs lors du démantèlement des sépultures. Il apparaît difficile d'isoler un seul de ces facteurs, tous ayant pu agir à des instants différents sur une même coquille. Cette typologie a pu être additionnée de 
l'étude localisée des modifications des états de surface. Sur certaines coquilles, il a été difficile de différencier les polis liés à l'utilisation de ceux liés à l'érosion marine. En effet, certains stigmates localisés d'origine anthropique se sont surimposés aux modifications de surface naturelles. Cependant, pour au moins $65 \%$ des nasses réticulées l'intégralité de la surface est polie et se traduit par effacement des ornementations des côtes (fig. 18B1). Ainsi la majorité des nasses présente des modifications attribuables à l'érosion marine et ont donc été ramassées après leur mort. La matière première ramassée par les Mésolithiques pour confectionner des parures était donc sous la forme de coquilles mortes échouées dans les laisses de haute mer. Le fort degré d'érosion observé sur le matériel de La Vergne a aussi pu être un critère de leur sélection. Celui-ci induit le fait que certaines de ces coquilles étaient peut-être prêtes à l'emploi dès leur ramassage sur la plage.

Fig. 20 - Application de la typologie de dégradation des nasses réticulées de La Vergne et de deux référentiels actuels.
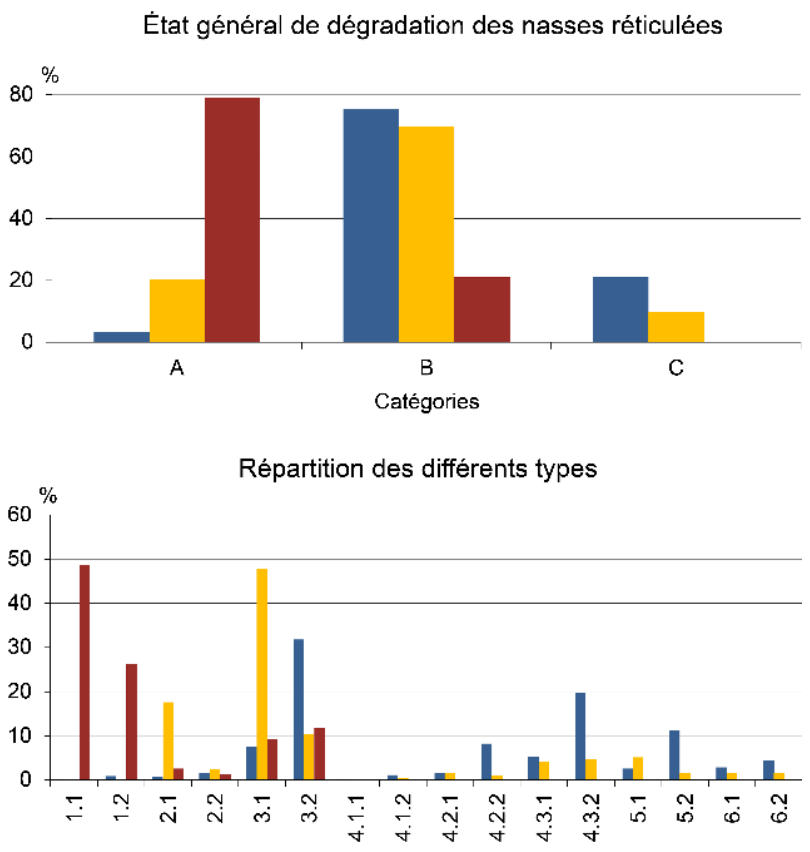

- La Vergne ( $\mathrm{N}=2105)$

11. Lot 1 Référentiels actuels $(N=193)$

- Lot 2 Réfèrentiels actuels $(N=76)$

LOT $\mathbf{N}^{\circ}$ 1. Pénestin près de l'estuaire de la Vilaine ; LOT $\mathbf{N}^{\circ} \mathbf{2}$. Polder de Sébastopol sur lîlle de Noirmoutier.

DAO C. Dupont.

\section{De l'érosion marine au percement de la coquille}

51 Ainsi, il est probable au vu du taux d'usure de certains gastéropodes et de la localisation de la perforation que certaines d'entre elles étaient déjà percées lors de leur ramassage comme cela s'observe actuellement sur les plages au niveau du dernier tour de spire pour les nasses et au niveau de l'apex pour les patelles (fig. 18A1-B2). L'érosion marine est sans doute aussi un des facteurs naturels qui a pu percer certains crochets de bivalve (Cabral et Martins 2016). Comme nous l'avons déjà décrit précédemment, l'état général de certaines valves atteste de ce fort taux d'usure (fig. 17). Mais d'autres 
critères plaident en la faveur de cette hypothèse : la localisation et la forme de la perforation (fig. 21B-C).

Fig. 21 - Exemples de perforations naturelles identifiées dans les parures de La Vergne (A1, B1, C1) et sur des référentiels actuels et archéologiques (A2, B2, C2).

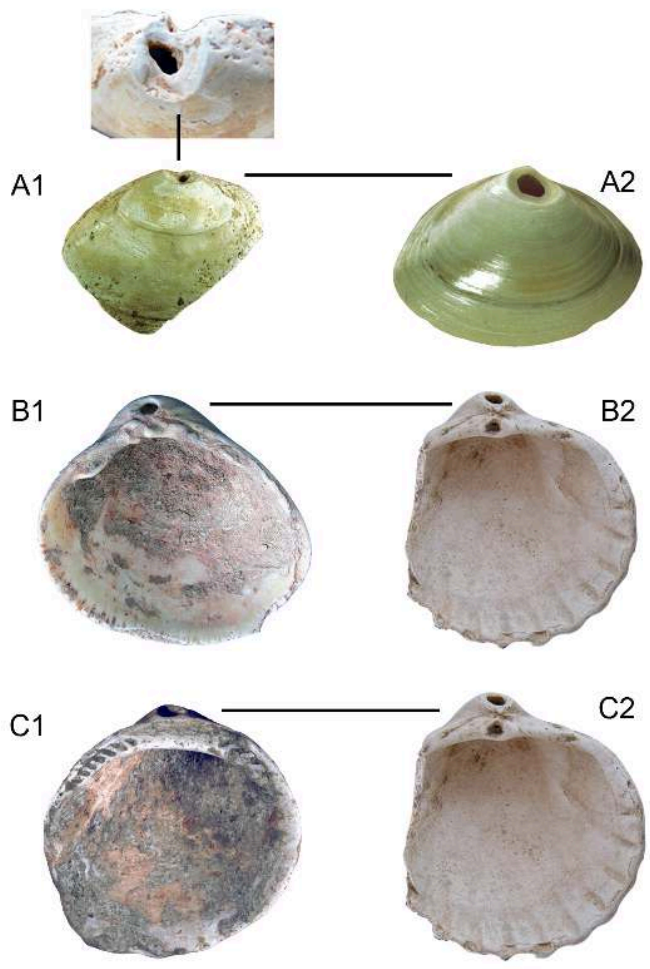

A1, A2. Perforations en forme d'entonnoir liée à un gastéropode perceur sur des spisules $(L=21$ et $30 \mathrm{~mm}$ ). Les critères associant cette perforation à son origine naturel sont le choix de l'espèce, sa localisation au niveau du crochet, sa forme en entonnoir et son diamètre ; B, c. Perforations liées à l'érosion marine ; B1. Bucarde de Norvège $(L=54 \mathrm{~mm})$; B2, c2. Bucarde $(62 \mathrm{~mm})$; c1. Amande de mer $(\mathrm{L}=51 \mathrm{~mm}$, l'intégralité du contour est modifié et le relief des dents de la charnière est moins accentué par rapport à des exemplaires vivants.

Photos et DAO C. Dupont.

Le percement par des organismes lithophages (algues, bryozoaires, annélides, cirripèdes, mollusques) est une autre piste d'origine naturelle envisagée pour certaines coquilles (Rolin 1971, p. 6, Boekshote 1966, Cabral et Monteiro-Rodrigues 2015). Sous le terme de lithophages ont été réunis les lithophages stricts qui attaquent uniquement le test des coquilles pour s'y réfugier et vivent dans la roche se protégeant ainsi contre l'environnement et les prédateurs dont le but est de se nourrir des parties molles (Rolin 1971, p. 6, Bishop 1975). On peut très bien imaginer que les coquilles ainsi percées ont pu servir de parure par la suite. Chaque espèce de lithophage possède une technique de perforation qui lui est propre. La morphologie des perforations variera en fonction de l'espèce incriminée (Rolin 1971, p. 9, Cabral et Monteiro-Rodrigues 2015). Le percement de tests par les lithophages provoque certaines stries caractéristiques (d'Errico et al. 1993, Cabral et Monteiro-Rodrigues 2015). Cependant, il apparaît que ces stries disparaissent une fois soumises à l'action marine (d'Errico et al. 1993). La forme des perforations peut nous permettre d'envisager une action par les lithophages. Des observations ont été faites afin de caractériser la forme de telles perforations (d'Errico et al. 1993, p. 252, Taborin 1993). Selon les espèces elle sera cylindrique, cylindrique 
convexe ou conique (Barge-Mahieu et Taborin 1991, p. 13, d'Errico et al. 1993, p. 246, Rolin 1971, p. 9, Cabral et Monteiro-Rodrigues 2015). Bien que F. d'Errico fait de l'emplacement un critère à ne pas « retenir pour distinguer les perforations dues à des prédateurs de celles qui sont dues à d'autres facteurs » (d'Errico et al. 1993), certains auteurs ont cité certains emplacements de la coquille touchés par les lithophages (Cabral et Monteiro-Rodrigues 2015). Nous avons pu observer cette caractéristique à de multiples reprises sur les échantillons actuels échoués sur les plages atlantiques. Les perforations obtenues sont reconnaissables par leur emplacement privilégié au niveau de l'umbo et leur forme en entonnoir parfaitement régulière. Ce type de stigmate a été observé sur au moins une valve de spisule (fig. 21A).

Fig. 22 - Comparaison des espèces majoritaires et minoritaires de la parure de La Vergne avec les espèces les plus représentées dans le régime alimentaire des populations mésolithiques.

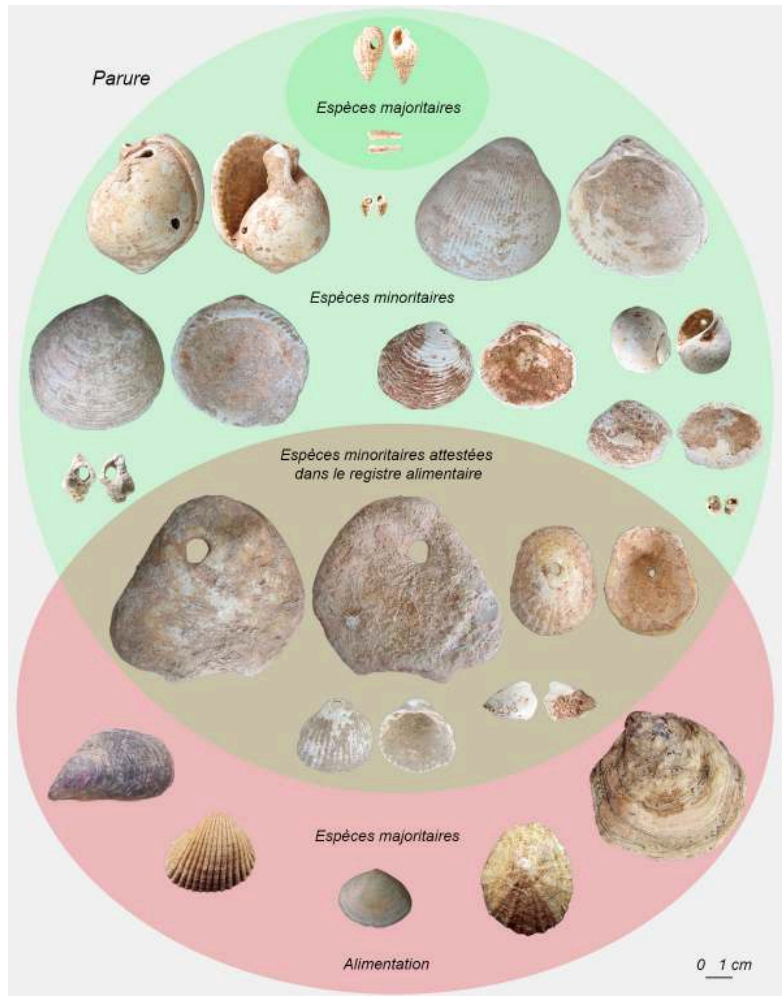

Photos et DAO C. Dupont.

Ainsi, il apparaît qu'au moins une partie des coquilles ramassées sur la plage pour constituer les parures de La Vergne était déjà percée. Ce résultat est important pour notre connaissance des populations mésolithiques et de l'activité qui consiste à rechercher de la matière première. La quête de coquille se faisait sur une anticipation de la matière première et était sans doute une activité qui se différenciait de la recherche de nourriture. On peut imaginer que cette activité était suffisamment importante pour ces groupes humains pour qu'ils la réalisent en parallèle de la recherche de nourriture. La comparaison des espèces majoritairement utilisées dans la parure de La Vergne avec celles qui sont connues dans le registre alimentaire traduit cette opposition (fig. 22). Les quelques exemplaires de valve d'huitre et de patelles de La Vergne présentent des indices d'érosion qui montrent que ces mollusques n'avaient pas été consommés avant d'avoir été utilisés pour servir de parure. Cette observation 
semble être une constante à la fois pour la parure du Mésolithique mais aussi pour d'autres utilisations des coquilles ramassées échouées en tant que matière première au fil de la chronologie, comme si les aliments étaient dissociés des autres activités (Dupont 2019).

Le degré d'usure général des tests ainsi que l'emplacement des perforations permettent de conclure à une collecte de la plupart de ces coquilles échouées mortes ainsi qu'à la présence, pour une partie d'entre eux difficilement quantifiable, de perforations sans doute naturelles. De plus, les effets des différents acteurs de la taphonomie nous livrent des coquilles dont la surface est blanche. Il ne faut pas perdre de vue que la coloration des tests coquilliers a très bien pu agir au niveau de la sélection de ceux-ci pour confectionner la parure (fig. 23). Malgré cette apparente uniformité de couleur et de composition de la parure de La Vergne en dentales et nasses réticulées la composition des différentes structures nous ouvre la voie vers d'autres pistes de différenciation de la parure à l'échelle des sépultures.

Fig. 23 - Comparaison des couleurs des coquilles de La Vergne (à droite) avec des homologues actuels.

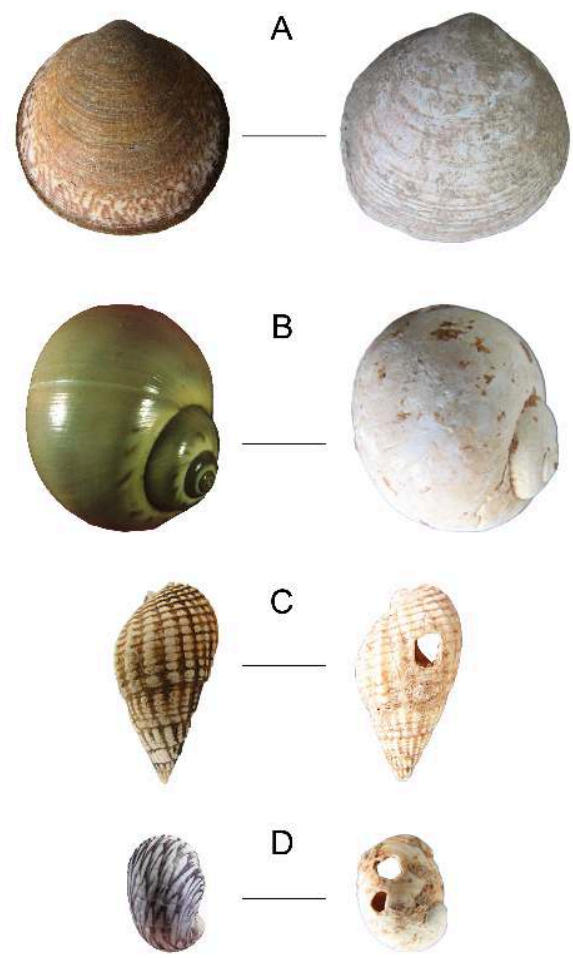

A. Amande de mer $(L=48$ et $51 \mathrm{~mm})$; B. Natice $(L=30$ et $25 \mathrm{~mm})$; c. Nasse réticulée $(L=25$ et $22 \mathrm{~mm})$; D. Nérite des rivières $(L=9$ et $8 \mathrm{~mm})$.

Photos et DAO C. Dupont.

\section{Différenciation des spectres malacofauniques en fonction des fosses sépulcrales}

Comme nous avons pu le voir lors de la présentation du site, les structures de celui-ci représentent à la fois des structures funéraires (St. 3, 7, 10 et 11) et des structures liées à l'activité gauloise (St. 1, 2 et 12). Les comparaisons ont uniquement été effectuées par 
structures funéraires sans tenir compte de la répartition des coquilles au sein de chacune d'elles, ce qui sera présenté dans une partie suivante. Les structures funéraires se dégagent largement par la présence de la malacofaune : plus de 400 restes chacune (fig. 24). Seule la structure funéraire 11 est mal fournie en nombre de restes avec seulement 5 restes. Cela peut s'expliquer par la nature de cette structure. Il s'agit d'un dépôt tardif constitué uniquement par les restes d'un squelette qui est de plus amputé par les structures 1 et 2 . Les structures gauloises possèdent également un peu de malacofaune. Ceci est dû à des apports extérieurs provoqués par l'effondrement de structures funéraires voisines. La structure 7 domine largement en nombre de coquilles, puis vient la structure 3 qui a été largement amputée, enfin la structure 10. Cette différence s'explique soit par le nombre de squelettes présents dans chaque fosse, soit par le degré de dégradation, mais aussi par une abondance sans doute différente des parures associées aux défunts. La structure 11 étant pauvre en parure et très amputée et les structures 1,2 , et 12 étant remaniés, seules les trois principales structures funéraires seront prises en compte (St. 3, 7 et 10) dans la suite de cette étude. Le nombre important de coquilles au sein de chacune d'entre elles en permet la comparaison.

Fig. 24 - Spectres malacofauniques des différentes structures de La Vergne.

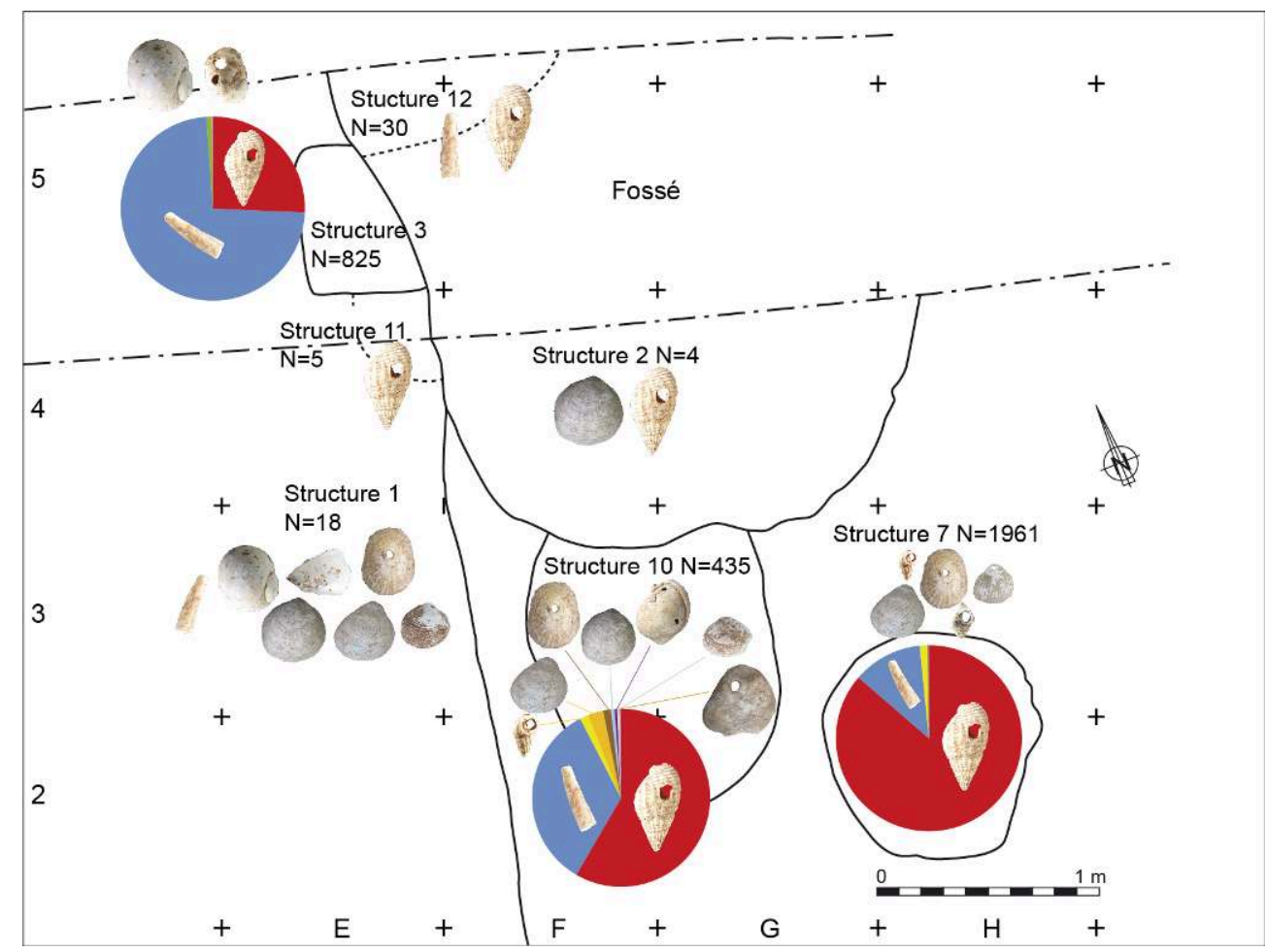

DAO C. Dupont.

La richesse spécifique des structures n'est pas corrélée aux nombres de restes (fig. 24). La structure 10, qui a un nombre de restes légèrement supérieur à 400 possède, à elle seule, 9 espèces. La structure 7 , qui a plus de 1800 restes, est composée de 7 espèces. Tandis que la structure 3 n'a que 3 espèces représentées pour 825 restes décomptés. Ces chiffres sont un premier indice de l'hétérogénéité de la composition des parures en coquilles de ces structures. Un autre fait marquant peut être observé : la structure 3 se différencie des deux autres structures funéraires et du spectre général de la 
malacofaune par une proportion plus importante de Dentalium sp. que de Tritia reticulata (fig. 24). Les structures 7 et 10 suivent, quant à elles le schéma général de la malacofaune décrit à l'échelle du site, dans lequel deux espèces sont majoritaires avec une proportion plus importante de nasses réticulées. La répartition des espèces minoritaires en fonction des structures est un autre caractère qui différencie les structures. Certaines espèces ne sont représentées que dans la structure $10:$ la spisule Spisula sp., le casque Semicassis saburon et l'huître plate Ostrea edulis.

Cette différenciation ne semble pas liée à la présente fortuite de coquilles isolées car les deux premières espèces sont représentées par deux exemplaires chacune. D'autres espèces sont présentes en majorité dans cette structure : 3 valves d'amandes de mer Glycymeris glycymeris, 9 de bucarde de Norvège Laevicardium crassum, 6 patelles Patella sp. Quelques coquilles de Tritia incrassata $(\mathrm{N}=7)$ y sont présentes. La structure 10 possède donc une grande richesse d'espèces $(\mathrm{N}=9)$ et plusieurs d'entre elles sont de grande taille. La structure 3 possède à l'inverse une faible richesse spécifique $(\mathrm{N}=4)$ et est composée d'espèces de taille moyenne comme les natices Euspira catena. Les deux nérites des rivières identifiées à La Vergne l'ont été dans cette sépulture. Enfin, la structure 7 possède sept espèces et deux d'entre elles, de taille moyenne, ne sont présentes que dans cette sépulture. Il s'agit d'une valve de coque Cerastoderma sp. et d'un murex Ocenebra erinaceus. Cette structure possède également deux patelles et 14 nasses Tritia incrassata. La structure 7 possède donc des espèces de taille petite à moyenne.

À travers la description de la composition des différentes structures funéraires, la diversité de la malacofaune s'y dessine par les espèces représentées, leur richesse et la taille de chacune d'entre-elles. Elle met donc en évidence une différenciation des structures funéraires du site de La Vergne. Nous verrons dans la partie qui suit que ces critères ne sont pas les seuls qui différencient les sépultures les unes des autres.

\section{Conclusion}

Les coquilles observées dans les sépultures mésolithiques de La Vergne appartiennent à 18 espèces différentes. Deux espèces de petite taille ont été utilisées en majorité la nasse réticulée et la dentale. Leur nombre diffère des coquilles de grande taille qui sont, elles, peu nombreuses.

Toutes les espèces proviennent de la mer à l'exception de Theodoxus fluviatilis inféodée à des milieux saumâtres à fluviatiles. Celle-ci a très bien pu être recueillie dans la Paléocharente ou ses affluents non loin du site. La présence et le profil de l'estuaire de la Paléocharente permettaient à l'influence marine de pénétrer à l'intérieur des terres. Les coquilles marines ont pu être ramassées à moins d'une trentaine de kilomètres du site d'après les données paléoenvironnementales connues dans la région. Cette hypothèse est corroborée par les mesures biométriques réalisées sur les nasses réticulées de La Vergne. En effet parmi celles-ci, une grande proportion se rapproche de l'espèce Tritia nitida inféodée aux côtes de mode abrité. Cette espèce a donc pu être ramassée sur les rives ensablées voire envasées de l'estuaire de la Charente. Le substrat sableux d'origine de la plupart des espèces identifiées ainsi leurs répartitions biogéographiques vont aussi dans ce sens. L'approvisionnement en matière première par les hommes et femmes de La Vergne a pu se faire aussi bien à l'issue de courts déplacements, que d'échanges avec d'autres groupes humains. 
61 La présence de coquilles subtidales additionnée de modifications attribuables à l'érosion marine post-mortem montre qu'une partie des coquilles a sûrement été recueillie échouées sur la plage et déjà dépourvues de la chair de l'animal. Elles ne sont sans doute pas issues du recyclage de coquilles de fruits de mer précédemment consommés. À ce résultat s'ajoute le fait que les coquilles marines utilisées à La Vergne en majorité ne sont pas connues pour avoir été consommées au Mésolithique. Les coquilles ramassées vides et déjà dépourvues de chair n'auraient ainsi pas la même destinée que les coquillages vivants. Cette observation fait écho aux écrits de Claude Lévi-Strauss « Il ne suffit pas qu'un aliment soit bon à manger, encore faut-il qu'il soit bon à penser" (Lévi-Strauss 1962). Si elle semble s'appliquer aux mollusques consommés, elle pourrait bien être déclinée pour les coquilles vides utilisées: Il ne suffit pas qu'une coquille vide soit bonne à décorer, encore faut-il qu'elle soit bonne à penser.

62 Ainsi, si certains facteurs biologiques agissant sur la répartition biogéographique des mollusques marins ont pu guider le choix des coquilles sélectionnées et utilisées par les hommes et femmes de La Vergne, d'autres critères d'ordre culturel ont aussi pu guider la main des êtres humains lors de leur ramassage sur la plage. Cette activité anticipait l'utilisation future de la matière première sélectionnée, comme en témoigne la présence de perforations naturelles utilisées pour les fixer.

63 À ce stade de l'analyse, la comparaison des différentes structures funéraires montre qu'elles se différencient par les quantités de coquilles utilisées, la richesse spécifique et les espèces elles-mêmes représentées. Ainsi, certaines espèces de grande taille, bien que faiblement représentées en nombre de restes, ne semblent pas anodines. Nous verrons dans ce qui suit que la diversité des sépultures de La Vergne ne s'arrête pas à ces seules observations.

\section{Des objets en coquille à la parure : actions anthropiques et traces d'usures}

De façon à éviter toute interprétation hâtive nous nous sommes attachés à décrire puis à codifier chacun des stigmates observés, indépendamment de la cause qui les a produits (façonnage, usure naturelle ou liée au port de ces objets). Pour les gastéropodes, l'emplacement de ces stigmates sur la coquille a été noté conformément aux propositions d'Y. Taborin (1993). La coquille, utilisée comme élément de parure, est orientée dans son sens de suspension (Barge-Mahieu 1991, p. 2), bien que ce dernier soit un peu théorique comme nous le verrons par la suite. On distingue donc la dernière spire, qui forme l'essentiel des surfaces disponibles, des spires inférieures. Sur cette dernière spire, le bord du labre, le bord de la columelle et le canal siphonal constituent des emplacements particuliers, facilement identifiables. Sur l'ensemble étudié il nous a paru intéressant de distinguer le haut, le bas et le milieu du labre, comme la partie supérieure et inférieure du bord columellaire qui peuvent notamment présenter des traces d'usure différentes. Pour les mêmes raisons, les zones E1, E2, E3, E4 et E5 définies par Y. Taborin sur la dernière spire ont chacune été divisées en deux parties inférieures et supérieures. Sur les coquilles de nasse, beaucoup plus allongées que les gastéropodes utilisés par cette auteure pour définir son zonage, nous avons été amenés à rassembler sous un même label les stigmates présents en E3 et en E4 (fig. 25). 
Fig. 25 - Découpage du test des gastéropodes turbinés en zones distinctes, et de la perforation en 8 cadrans.

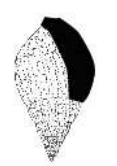

E1

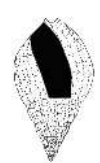

E2

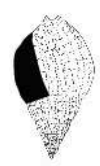

E3-4

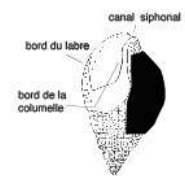

E5

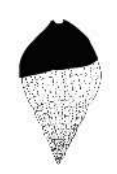

Partie supérieure de la dernière spire

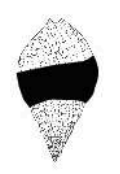

Partie inférieure de la dernière spire

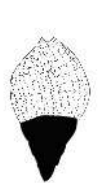

Spires inférieures

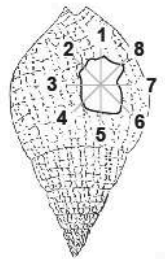

Zonage de la perforation

D’après Taborin 1984, 1993 ; DAO L. Quesnel et L. Laporte.

De telles surfaces, lorsqu'elles ne sont pas altérées, peuvent porter un lustré, un poli, une abrasion ou une patine. Nous avons utilisé le terme «lustré » lorsque la coquille présente un aspect luisant sans modification sensible de son relief. Le terme " poli " s'applique uniquement lorsque le relief de l'ornementation de la coquille est atténué. Celui de "patine " a été réservé aux cas peu nombreux où la surface de la coquille possède à cet endroit un cale lisse et de couleur ivoire (fig. 26A). "L'abrasion » enfin entame la surface de la coquille et produit un aplanissement de sa surface. Pour chaque coquille de gastéropode ces critères nous ont permis de décrire 12 zones distinctes.

Fig. 26 - Principaux types de stigmates observés sur les coquilles.

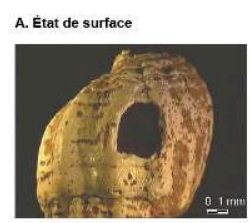

Altération

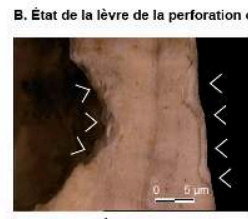

Écaillures

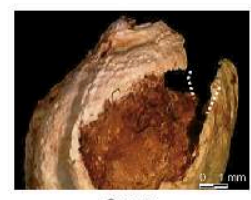

cossure

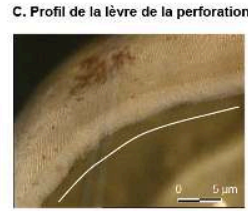

Découpe

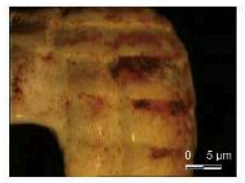

Lustré

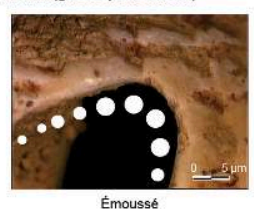

Émoussé
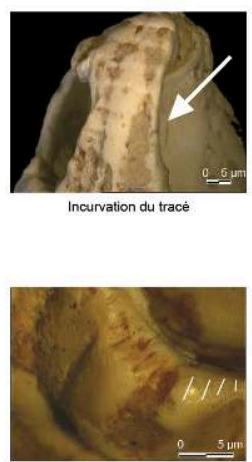

Strie
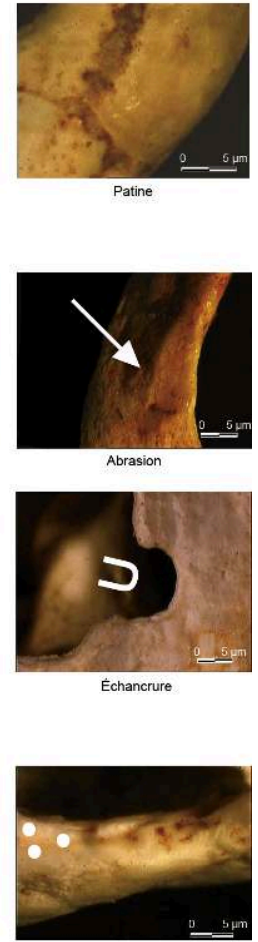

Ponctuations

Clichés L. Laporte et J.-Y Lefeuvre. Sauf mention contraire, toutes les photos sur les figures de cette partie montrent des coquilles recueillies dans les sépultures de La Vergne. 
vre du labre mais aussi celle de la perforation portent des stigmates un peu différents. On peut y observer un émoussé voire une abrasion, des écaillures voire une cassure, une incurvation de son tracé, voire une véritable échancrure. L'abrasion, comme dans le cas précédent, correspond à un enlèvement de matière qui produit une surface plane. Une lèvre "émoussée » présente un aspect luisant qui s'accompagne d'une modification du profil initial. Lorsque ce stigmate localisé est encore plus prononcé cela peut conduire à une légère déformation du tracé de la lèvre. Nous avons alors utilisé le terme " incurvation ». Le terme « écaillures » a été employé lorsque l'on observe de petits enlèvements sur le bord de la lèvre, sans toutefois que son tracé en soit sensiblement modifié. Le bord de la lèvre pouvait également être cassé à cet endroit. Parfois, lorsque l'action qui a provoqué l'un ou l'autre de ces précédents stigmates est plus accentuée en un point précis, elle provoque alors une forte déformation localisée du tracé de la lèvre. Dans ce cas-là nous avons employé le terme " d'échancrure » (fig. 26B). Bien entendu il existe un continuum entre une lèvre écaillée ou émoussée, puis cassée ou présentant une incurvation du tracé, voire échancrée. Malgré leur caractère empirique, ces définitions nous ont permis de décrire 11 points d'observation supplémentaires sur chaque pièce, ce qu'il faut multiplier par le nombre de coquilles observées qui est de l'ordre de 1300 individus. Quant au millier de dentales étudiés, 3 points d'observation ont été retenus: le test, l'extrémité antérieure et postérieure.

67 Pour ce qui est de la perforation, quel que soit le type de coquille qui la porte, nous avons retenu un certain nombre de critères supplémentaires, au premier rang desquels figure sa localisation. D'autres concernent sa forme, la régularité du contour ou son état de conservation. La longueur de la perforation a été mesurée en suivant l'axe de symétrie de la coquille, et sa largeur selon l'axe perpendiculaire. Nous avons divisé le contour de la perforation en 8 cadrans, selon un procédé également proposé par Y. Taborin (Gaillard et al. 1984). Lorsque la perforation présente un axe d'étirement privilégié ce dernier a été indiqué. Pour chacun de ces 8 points nous avons décrit le profil de la lèvre et noté les traces d'usure comme les stigmates liés à une action technique. Le profil de la perforation peut être plat, arrondi, aplati vers l'extérieur, incurvé vers l'intérieur ou biseauté. Des critères similaires à ceux utilisés sur la lèvre du labre ont été retenus pour décrire les traces d'usure. Présents en grand nombre sur la lèvre des perforations anthropiques et recouvrant bien souvent les stigmates liés à une action technique, ces critères ont ici été interprétés directement en termes de traces d'usure. Lorsque ces dernières permettaient de localiser précisément l'emplacement du passage d'un lien, ce dernier point a également été indiqué.

armi les stigmates qui résultent d'une action technique, nous avons reconnu des écaillures, des ponctuations, des stries, des traces d'abrasion ou de découpe (fig. 26C). Elles rendent compte de différentes actions techniques telles que la percussion, le piquetage, auxquels s'ajoutent le rainurage, l'abrasion ou le raclage selon la terminologie proposée par Averbouh et Provenzo (Julien et al. 1999) pour des supports en os ou en bois de cerf. Dans quelques rares cas l'usage d'un foret rotatif est attesté. Il se caractérise par les stries concentriques laissées par le foret sur le bord et sur la lèvre de la perforation. Suivant l'exemple proposé par Y.Taborin (1993), nous avons décomposé la chaîne opératoire qui a abouti au percement de la coquille en trois étapes, chacune faisant appel à une ou plusieurs actions différentes : la préparation de la surface qui va recevoir la perforation, puis la perforation proprement dite, 
éventuellement suivie d'une reprise destinée à en régulariser le contour. Les stigmates correspondants peuvent être situés sur la lèvre de la perforation ou sur les surfaces externes de la coquille immédiatement attenantes. Malheureusement ils sont souvent estompés, voire effacés, par les traces d'usure ou l'altération du test.

Pour chaque pièce, si l'on prend maintenant l'ensemble de la coquille, certaines présentent visiblement des traces d'usure beaucoup plus accentuées que d'autres. Nous avons alors tenté de codifier cette première impression afin de pouvoir comparer chaque lot, voire la parure associée à chaque individu, en particulier en ce qui concerne les nasses percées. La présence d'une échancrure de la lèvre, d'une patine ou d'une abrasion des surfaces est la marque d'une usure prononcée en un point donné de la coquille (indice 2). Quelques écaillures, un émoussé ou une simple incurvation du tracé de la lèvre, tout comme la présence d'une plage polie localisée sur le test, marquent un degré d'usure plus limité (indice 1). Lorsqu'aucun de ces stigmates n'était présent, nous avons conclu à l'absence de traces d'usure (indice 0 ). Sur chaque pièce, nous avons retenu cinq zones (E1, E5, le labre, la columelle, la lèvre de la perforation) auxquelles nous avons attribué le degré d'usure le plus élevé observé à cet endroit. Puis nous avons fait la somme des indices propres à chaque zone. Ce nouvel indice caractérise le degré d'usure de la pièce. Plus il sera élevé, plus la pièce présentera une usure prononcée. L'usure prononcée de certaines parures déposées en contexte funéraire est un fait remarquable qu'il conviendra ensuite d'interpréter en fonction de leur association avec des individus d'âges différents.

\section{Stigmates observés sur les différentes espèces de coquillage utilisées}

\section{Scaphopodes}

\section{Dentales Dentalium $s p$.}

Un peu plus d'un millier de dentales ont été recueillis dont 600 proviennent de la structure 3, 240 de la structure 7 et 150 de la structure 10. La vingtaine d'exemplaires restant se répartit entre les structures 1 et 12 .

Pour ce qui est des fragments de dentales provenant des sépultures de La Vergne, $95 \%$ sont d'une taille inférieure à $15 \mathrm{~mm}$. La longueur moyenne de ces fragments est de $8 \mathrm{~mm}(8,7 \mathrm{~mm}$ pour la structure $3,7 \mathrm{~mm}$ pour les structures 7 et 10$)$, ce qui est très largement inférieur à la taille des exemplaires recueillis échoués sur l'estran (fig. 27). Le diamètre antérieur présente une valeur moyenne de $3,2 \mathrm{~mm}(3,4 \mathrm{~mm}$ pour la structure $2,8 \mathrm{~mm}$ pour les structures 7 et 10) qui est assez proche de celle du diamètre postérieur égale à $2,7 \mathrm{~mm}(2,9 \mathrm{~mm}$ pour la structure $2,4 \mathrm{~mm}$ pour les structures 7 et 10$)$. Ces valeurs sont également différentes de celles mesurées sur l'échantillon de référence (fig. 27). 
Fig. 27 - Taille des objets de parure en dentale de La Vergne $(N R=971)$ en comparaison de référentiels actuels $(N R=43)$ trouvés sur les plages de Vendée et du bassin d'Arcachon.
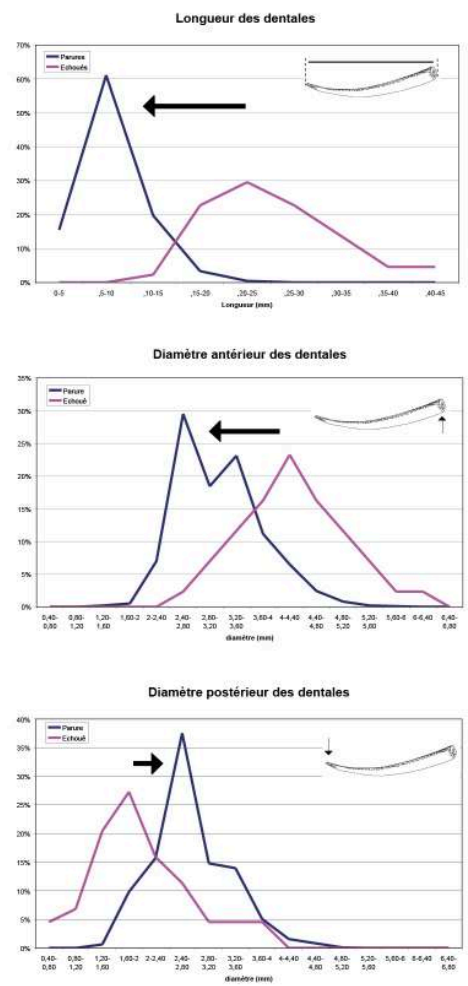

DAO L. Laporte et C. Dupont.

Il semble bien que les deux extrémités des dentales récoltés aient été sectionnées verticalement à leur longueur, dans la plupart des cas, afin d'obtenir un ou plusieurs petits tubes cylindriques de dimensions standardisées. De plus, cette opération présentait l'avantage de laisser passer un lien par une ouverture postérieure. Sur les exemplaires échoués celle-ci mesure à peine quelques dixièmes de millimètre de diamètre. Le diamètre de la lumière de l'ouverture postérieure peut donner une idée de la taille du lien utilisé. La plus petite valeur mesurée est de l'ordre de 1,5 $\mathrm{mm}$. Cependant aucune trace de façonnage n'a été observée sur ces échantillons. Il semble donc que la segmentation des coquilles ait été obtenue par flexion sans aucune préparation préalable. Les différences de dimension observées entre structures témoignent de possibles variabilités soit lors du raccourcissement des dentales, soit lors de leur utilisation. Elles peuvent aussi refléter une hétérogénéité de tailles des dentales en fonction de leurs positions dans les sépultures, ce que nous n'avons pas encore testé.

Un nombre important de dentales (80 exemplaires) ont été recueillis encastrés les uns dans les autres, comme autant d'éléments enfilés sur un lien (fig. 28). Nous n'avons cependant jamais observé plus de deux coquilles ainsi emboîtées successivement. Certains dentales emboîtés présentent une usure de la surface du test qui est de toute évidence postérieure à leur emboîtement; la partie du dentale recouverte par la coquille précédente possède des côtes intactes, alors que celle située à l'extérieur est polie. Remarquons également que de l'ocre est parfois présente entre deux coquilles emboîtées. Cette observation irait dans le sens d'une coloration des coquilles préalablement à leur emboîtement (st3 $\mathrm{n}^{\circ}$ 96). 
Fig. 28 - Deux dentales emboîtés. Remarquer l'échancrure du bord du deuxième dentale.

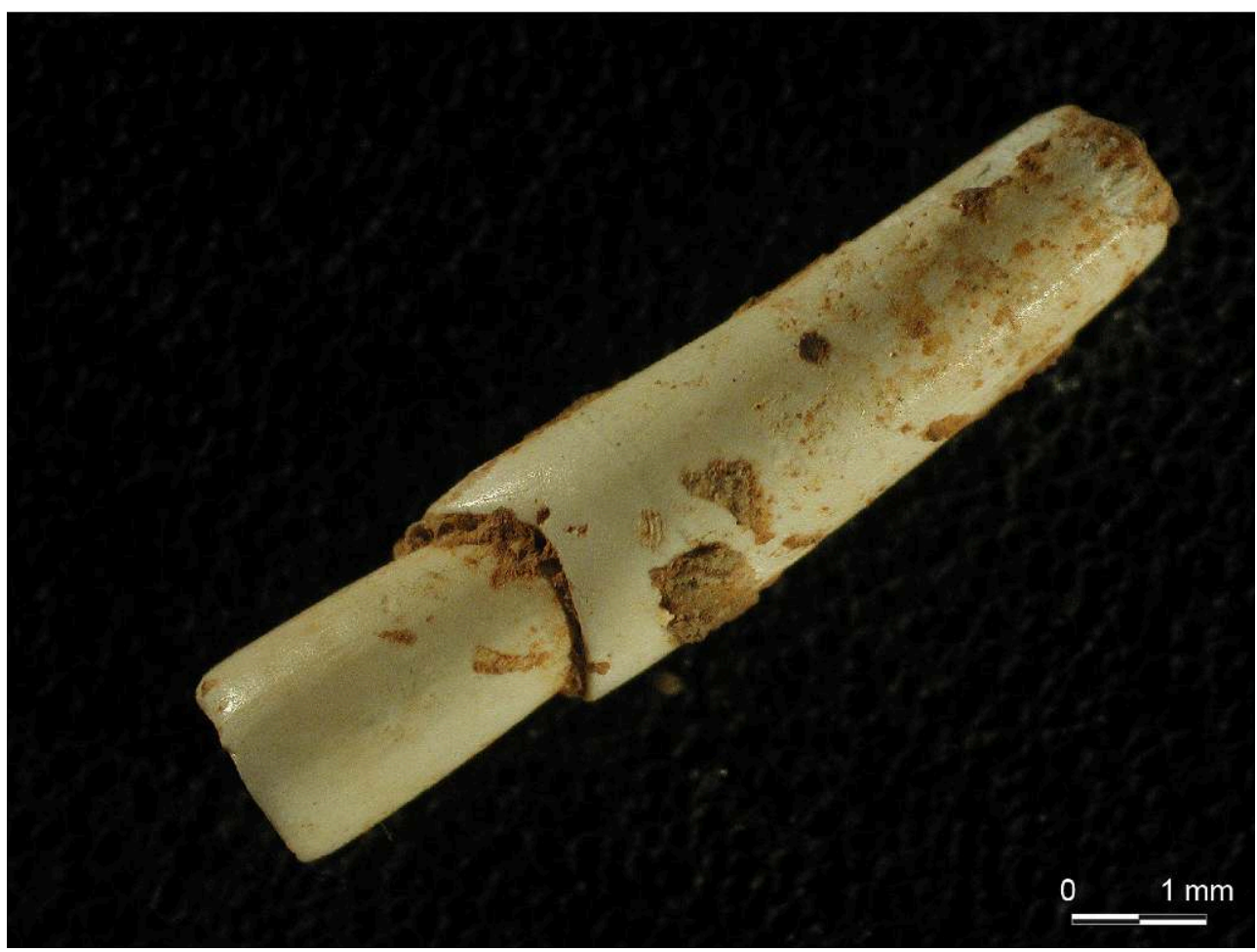

Cliché J.-Y. Lefeuvre.

Des traces d'usure ont également été repérées sur des coquilles isolées. Au moins $10 \%$ d'entre elles présentent de tels stigmates nettement marqués. Les traces d'usure affectent l'une ou l'autre des extrémités, et parfois la surface du test. Lorsqu'elles concernent une des extrémités, il peut s'agir soit d'un émoussé de la lèvre (76 exemplaires), soit d'une échancrure du tracé de la perforation (25 exemplaires). Dans le premier cas, la partie émoussée affecte tout le contour de la lèvre. Cet émoussé porte préférentiellement sur l'extrémité postérieure de la pièce (44 exemplaires), parfois sur les deux extrémités (19 exemplaires) et plus rarement sur la seule extrémité antérieure (13 exemplaires). Dans le second cas, l'échancrure peut résulter soit d'un écaillage localisé du bord externe de la lèvre, soit d'une partie émoussée plus accentuée à cet endroit. Elle pourrait être interprétée comme la trace d'un lien permettant de fixer la coquille sur un support en matière périssable.

L'usure du test semble un peu plus fréquente (137 exemplaires) que celle des extrémités. Elle correspond soit à un poli de toute ( 34 exemplaires) ou une partie (61 exemplaires) de la surface, soit à l'abrasion de l'une de ses faces (42 exemplaires). Cette usure, surtout représentée sur quelques dentales de la structure 3 , se traduit par l'effacement latéral des côtes et un aplanissement de la surface. Du fait de la courbure du dentale, cet aplanissement provoque aux extrémités une déformation du tracé de la lèvre. En général une seule extrémité est affectée, voire les deux sur au moins 9 exemplaires Cette déformation qui recoupe le bord du dentale en biseau, ne présente pas l'aspect émoussé ou écaillé des échancrures que nous venons de décrire. La déformation du tracé de la lèvre est également plus large que dans le cas précédent pour lequel nous réserverons le terme d'échancrure. Cette plage d'abrasion présente sur un seul côté du dentale pourrait résulter du frottement sur un support. Quelques 
rares coquilles présentent deux plages d'usure latérales jointes, comme si la coquille s'était légèrement décalée le long du support.

\section{Gastéropodes}

\section{Nasses réticulées Tritia reticulata}

Des coquilles de Tritia reticulata percées sont présentes dans toutes les structures archéologiques mais en nombre très variable. Elles constituent l'essentiel du corpus étudié avec près de 2000 pièces. Trois quarts d'entre elles proviennent de la structure 7 où elles sont représentées par un peu plus de 1600 individus. 250 coquilles percées de Tritia sp. proviennent également de la structure 10 et 210 exemplaires de la structure 3.

La plupart des coquilles ont été recueillies mortes sur l'estran, bien que quelques-unes aient pu être collectées vivantes. Nombre de ces coquilles roulées présentaient une ou plusieurs perforations naturelles. Certaines de ces perforations naturelles ont pu être utilisées en l'état pour le passage du lien. Mais le plus souvent le contour de la perforation a été régularisé sur toute sa circonférence à l'aide d'un instrument tranchant. Rien ne permet alors de les distinguer de perforations effectuées par la main d'une femme ou d'un homme (fig. 29). Dans tous les cas un seul de ces orifices semble être fonctionnel, bien que l'on ne puisse pas toujours exclure d'éventuels cas de réparations. La perforation utilisée pour le passage d'un lien est presque toujours située en E2 (90 \%), beaucoup plus rarement en E1 ou E3-E4 (4\% à $5 \%$ ), exceptionnellement en E5 (1 exemplaire ; fig. 30 ).

Fig. 29 - Nature et emplacement de la perforation sur les nasses.

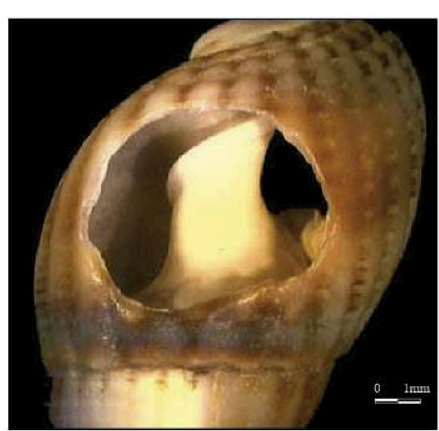

1. Perforation naturelle

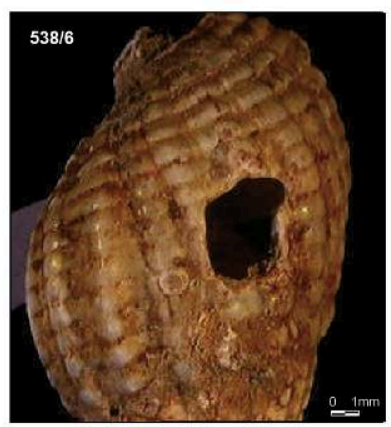

4. Perforation en E2

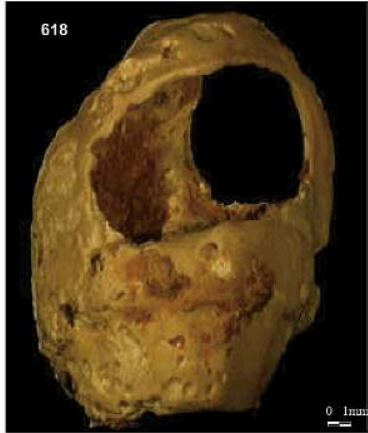

2. Reprise d'une perforation naturelle

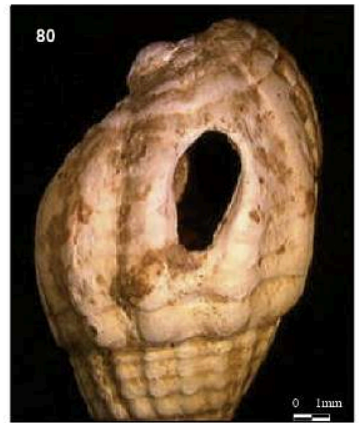

5. Perforation en E3-E4

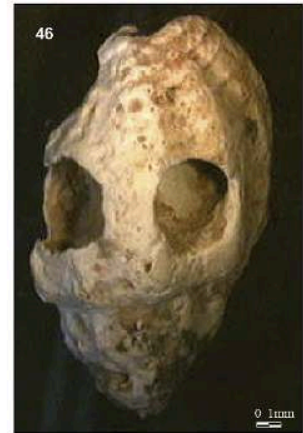

3. Perforations naturelle et anthropique

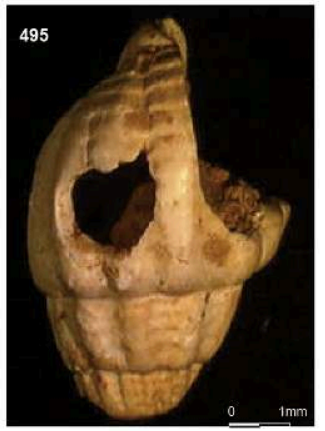

6. Perforation en E4 après cassure

Les numéros en haut à gauche de chaque photo renvoient au numéro d'inventaire de la pièce, tels qu'attribués au fur et à mesure de la fouille. II en va de même sur les figures suivantes.

Clichés L. Laporte et J.-Y Lefeuvre. 
Fig. 30 - Caractéristiques des perforations sur les nasses.

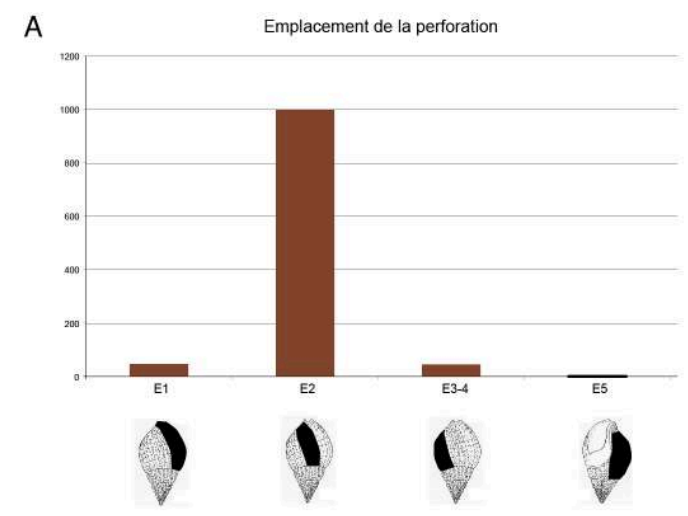

B

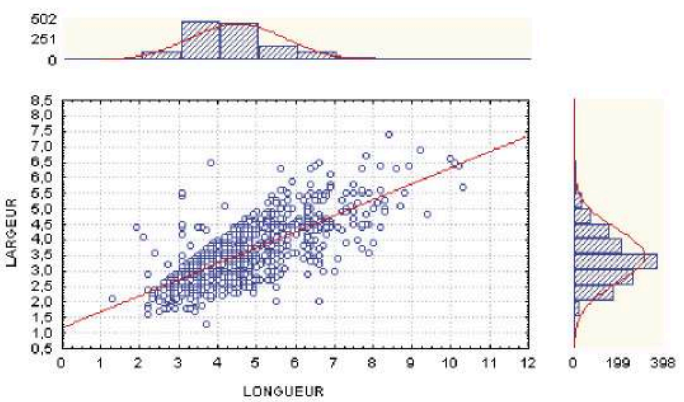

A. Emplacement de la perforation sur la coquille, exprimée en nombre de perforations sur l'axe des ordonnées (chaque pièce portant généralement une seule perforation, très rarement deux) ;

B. Dimensions de la perforation.

DAO L. Laporte.

Il existe cependant 3 exemplaires où l'on peut s'interroger sur le caractère fonctionnel de deux orifices situés sur la même coquille, le premier étant toujours situé en E2 alors que le second est respectivement en E3-4 ou en E5. La longueur de la perforation fait en moyenne $4,5 \mathrm{~mm}$, avec pour valeurs extrêmes $1,3 \mathrm{~mm}$ et $10 \mathrm{~mm}$. La largeur de la perforation fait en moyenne 3,5 mm avec pour valeurs extrêmes $1,3 \mathrm{~mm}$ et 7,5 $\mathrm{mm}$. Ces deux mesures sont d'ailleurs en général proportionnelles. Remarquons que dans les deux cas, la valeur la plus étroite de la perforation est de l'ordre de 1,5 mm, qui est également la valeur la plus petite pour l'orifice des dentales. Dans ce dernier cas, nous avions déjà envisagé qu'il pût s'agir du diamètre du lien le plus étroit destiné à être enfilé par cet orifice. La perforation est généralement de forme ovale (82\%), plus rarement quadrangulaire $(10 \%)$ ou circulaire $(8 \%)$. Le contour de la perforation n'est régulier que dans moins de $35 \%$ des cas.

Rappelons les trois étapes successives de la chaîne opératoire : la préparation de la surface qui va recevoir la perforation, le percement proprement dit, puis une régularisation du tracé de la lèvre de la perforation. Les différents stigmates correspondant à chacune de ces étapes sont difficiles à observer car ils furent le plus souvent effacés par les traces d'usure laissées par le frottement du lien contre la lèvre de la perforation. Les traces laissées lors de l'une ou l'autre de ces opérations ont été observées sur 490 pièces soit environ $30 \%$ du total. Cependant, les coquilles portant les traces d'une préparation de la perforation, la première étape de cette chaîne opératoire, ne représentent guère plus de $4 \%$ du total. 
80 Les stigmates rendant compte d'actes techniques mis en œuvre pour préparer la surface de la perforation sont l'incision (24 exemplaires) et surtout l'abrasion (38 exemplaires). Dans un ou deux cas une préparation de la perforation par piquetage peut être envisagée. Pour ce qui est des incisions, elles rendent compte d'au moins trois techniques différentes. Il peut s'agir de fines incisions parallèles destinées uniquement à régulariser la surface qui va recevoir la perforation. Dans ce cas il s'agit d'un raclage.

81 On trouve également deux incisions parallèles, plus profonde que les précédentes, qui délimitent ainsi deux des bords de ce qui deviendra la perforation (fig. 31). Cette dernière se caractérise par l'existence d'un ou deux bords rectilignes qui se prolongent ensuite par de petites encoches débordant de sa lèvre. Une troisième technique consiste à réaliser une unique et profonde incision par un mouvement de va-et-vient qui souvent débute la perforation de la coquille en son centre. On observe alors deux encoches bien marquées sur les bords opposés de la perforation. L'axe de ces deux encoches passe le plus souvent par le centre de la perforation et sa direction suit en général l'axe 4-8, plus rarement l'axe 1-5. Nous avons réservé le terme de rainurage à l'action technique qui provoque ces derniers stigmates (fig. 32). Un cas d'amorce de rainurage abandonnée en cours d'exécution est visible en E1 sur une coquille qui a ensuite été percée par simple percussion.

Fig. 31 - Préparation de la perforation (nasses), présence d'incisions sur le bord de la perforation. Les incisions observées sont clairement distinctes de toute autre forme d'altération de la coquille, notamment d'éventuelles altérations chimiques qui auraient pu mettre en valeur la structure cristallographique du coquillage, ce qui ici n'est pas le cas.

Préparation de la perforation
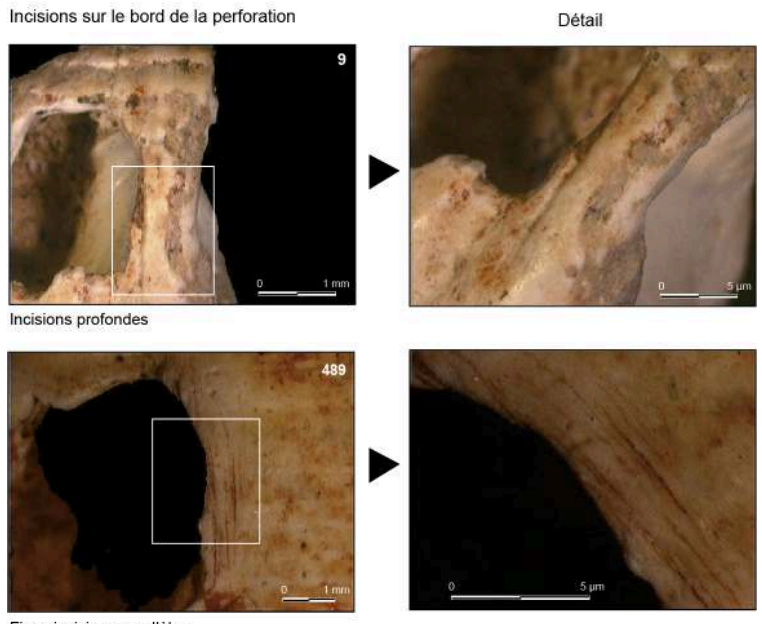

Fines incisions parallèles
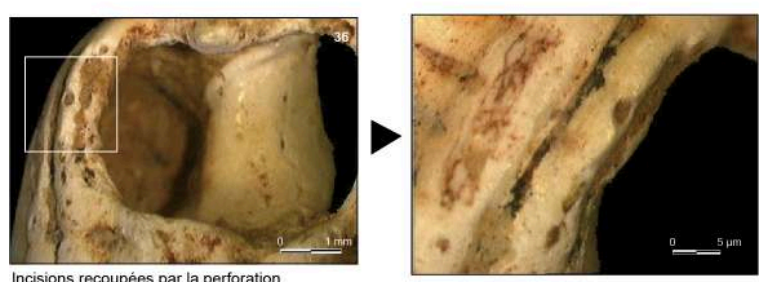

Clichés L. Laporte et J.-Y Lefeuvre. 
Fig. 32 - Préparation de la perforation (nasses), traces résultant d'un rainurage.

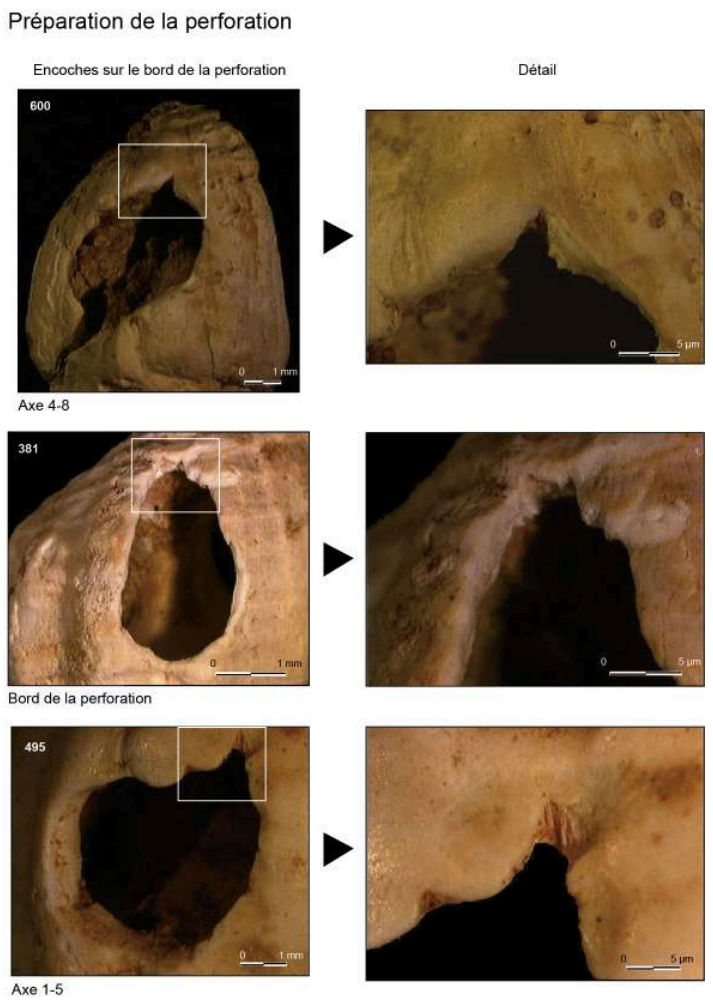

Clichés L. Laporte et J.-Y Lefeuvre.

Dans tous les cas, la perforation elle-même est obtenue par percussion, le plus souvent sur la face externe de la coquille. C'est du moins ce que suggèrent les écaillures qui ont souvent été observées sur la partie interne de la lèvre de la perforation; des débris de coquille ont d'ailleurs été retrouvés dans son prolongement à l'intérieur de quelques exemplaires. Nous avons noté un seul cas pouvant correspondre à une perforation à l'aide d'un foret rotatif, marqué par la présence de stries concentriques.

Le contour de la perforation est ensuite régularisé avec un instrument tranchant généralement utilisé dans un mouvement circulaire sur tout son pourtour en l'agrandissant. Les stigmates observés peuvent alors correspondre soit à un bord biseauté continu et bien délimité (19 exemplaires), soit à un bord plus irrégulier, biseauté sur sa face externe et présentant quelques écaillures sur sa face interne. Ce dernier cas est de loin le plus fréquent (179 exemplaires soit un peu moins de $80 \%$ des cas observés). Quelques stries localisées ont également été parfois observées (29 exemplaires).

Dans l'ensemble, la chaîne opératoire mise en œuvre présente peu de variantes. Les techniques utilisées pour préparer la surface de la perforation sont à la fois les plus difficiles à repérer et celles qui présentent la plus forte variabilité. En revanche, dans la très grande majorité des cas, la perforation elle-même est obtenue par percussion. Puis elle est agrandie avec un instrument tranchant dans un mouvement circulaire qui régularise ainsi son tracé et le profil de sa lèvre.

L'observation détaillée des traces d'usure porte sur plus d'un millier d'individus. Cellesci sont extrêmement fréquentes. $35 \%$ des pièces présentent une usure prononcée soit de la perforation, alors marquée par une échancrure bien marquée (qui peut également 
se retrouver sur le bord du labre), soit de la surface du test avec notamment la présence de plages d'abrasion. $55 \%$ des pièces possèdent des traces d'usure plus discrètes, comme une simple incurvation du tracé de la lèvre du labre, ou un poli en un point localisé de sa surface. Si l'on excepte les pièces trop altérées pour qu'une observation soit possible, seules $10 \%$ des coquilles ne portaient aucune trace d'usure. Ces dernières concernent à peu près toutes les parties de la coquille. Au niveau de la perforation nous avons vu qu'elles ont souvent effacé la plupart des traces de façonnage. Des traces d'usure sont également présentes sur la surface du test, sur le labre, sur le bord columellaire, et même parfois sur la base du canal siphonal. Il existe également au sein des mêmes lots, des coquilles qui ne présentent aucune trace d'usure particulière sans que cela puisse être mis sur le compte d'une altération différentielle.

Il n'est pas toujours aisé de différencier une usure naturelle de la surface du test de traces spécifiquement liées à son usage comme objet de parure. Seule une usure différentielle sur une partie précise de la coquille peut permettre d'avancer une hypothèse de ce genre. Les traces d'usure observées correspondent à une patine, à un poli ou à un lustré de la coquille, exceptionnellement à une abrasion de sa surface. Elles sont surtout accentuées en E1; zone de la coquille qui porte d'ailleurs les seules plages patinées observées. Cela pourrait être le fruit du frottement d'un lien à cet emplacement. La trace d'un lien reliant le labre à la perforation est d'ailleurs nettement marquée par une bande d'ocre rouge en E1 sur un exemplaire de la structure 10 (fig. 33). Cette hypothèse est en accord avec les traces d'usure observées sur la lèvre des perforations et sur le labre lui-même. Quelques plages d'abrasion assez étendues ont parfois été repérées en E5. Là elles pourraient être liées au frottement de cette partie bombée de la coquille contre un support en matière périssable. Si l'on prend maintenant un axe vertical, la partie inférieure de la dernière spire est celle qui porte les traces d'usure les plus accentuées. C'est également la partie la plus bombée de la coquille et donc, comme dans le cas précédent, celle qui a le plus de chance d'être affectée par le frottement contre un support (fig. 34). Signalons l'existence d'un cas particulier où les spires inférieures présentent un poli nettement plus accentué que le reste de la coquille. 
Fig. 33 - Traces d'usure en E1 sur les nasses percées.

Traces d'usure en E1
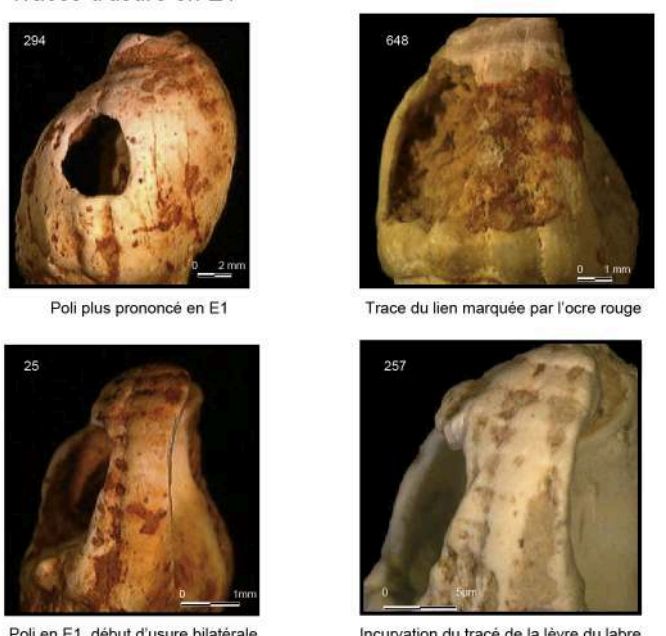

Poli en E1, début d'usure bilatérale

Incurvation du tracé de la lèvre du labre

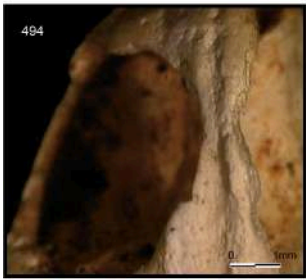

Rétrecissement par usure bilatérale

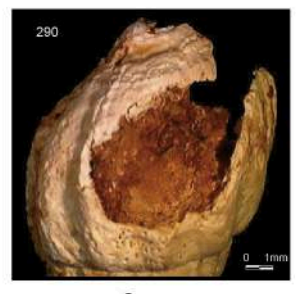

Cassure

Clichés L. Laporte et J.-Y Lefeuvre. 
Fig. 34 - Usure du test sur les nasses percées, exprimée en nombre de stigmates observés sur l'axe des ordonnées (différents stigmates ont pu être observés en différents points de chaque pièce).

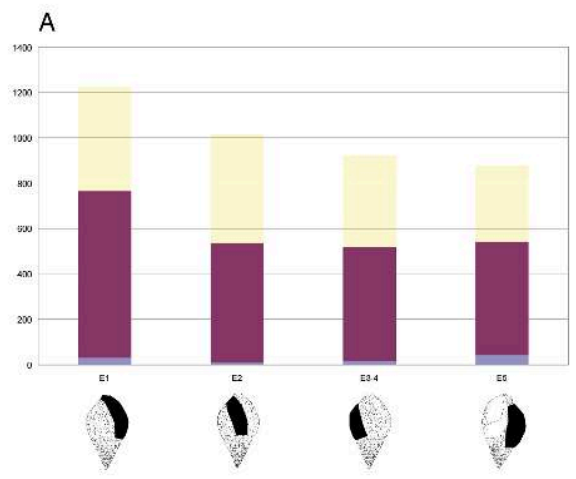

B

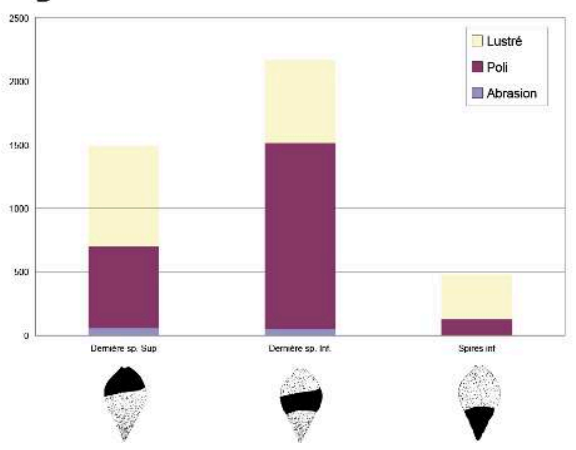

A. Dans le sens de la largeur ; B. Dans le sens de la hauteur. DAO L. Laporte.

87 Les traces d'usure sur le labre sont presque systématiques (fig. 35). Si l'on exclut les pièces trop altérées pour qu'une observation soit possible, près des $3 / 4$ des pièces présentent des traces d'usure sur le labre. Les parties émoussées ou présentant une abrasion de la lèvre s'étendent en général sur presque toute la longueur de cette dernière. Ce type d'usure peut, dans certains cas, aller jusqu'à une réduction du relief des petites dents présentes sur la partie interne du labre. Il représente suivant les lots, $70 \%$ à $90 \%$ des traces d'usure observées à cet endroit. Beaucoup plus rares sont en revanche, les écaillures du bord externe de la lèvre, l'incurvation ou l'échancrure du tracé de la lèvre, souvent très localisées sur chaque pièce prise séparément. Statistiquement, nous verrons que leur situation sur le haut, le milieu ou le bas du labre est variable suivant les lots étudiés (fig. 36). 
Fig. 35 - Traces d'usure sur le labre des nasses percées.

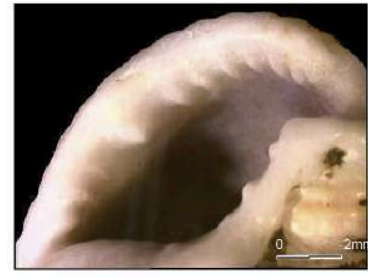

1. Coquille recueillie sur l'estan

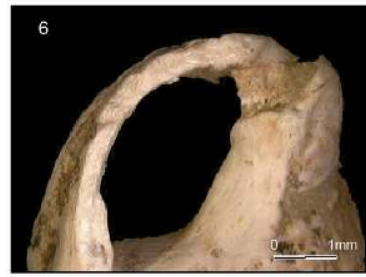

4. Forte abrasion du labre

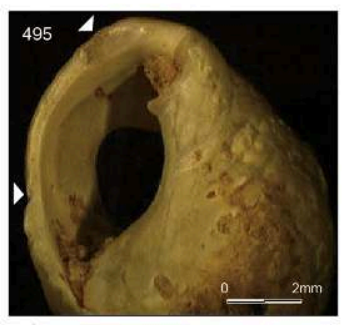

7. Écaillures postérieures à l'abrasion

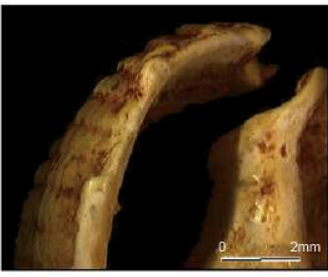

2. Cassure de la lèvre du labre

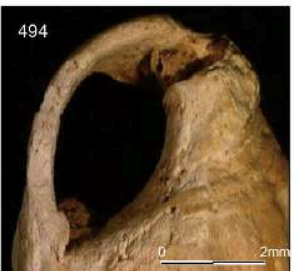

5. Abrasion du labre médian

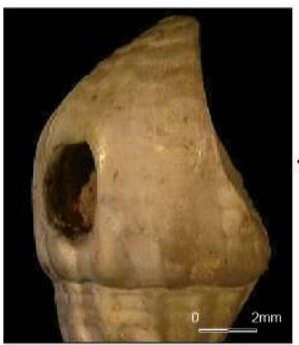

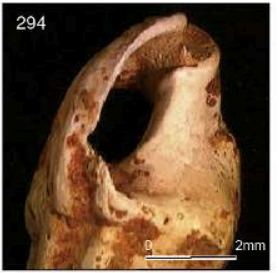

3. Échancrure de la lèvre du labre

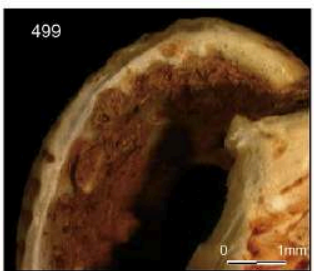

6. Émoussé du labre supérieur

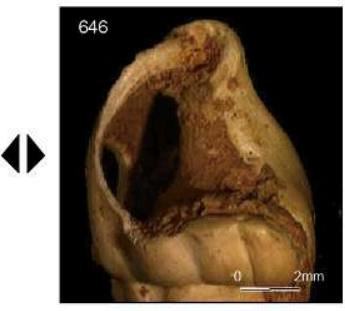

8. Façonnage par abrasion

Clichés L. Laporte et J.-Y Lefeuvre.

Fig. 36 - Types d'usure représentés sur le labre des nasses, exprimés (axe des ordonnées) en nombre de pièces sauf pour l'usure différentielle du labre qui est en pourcentage.
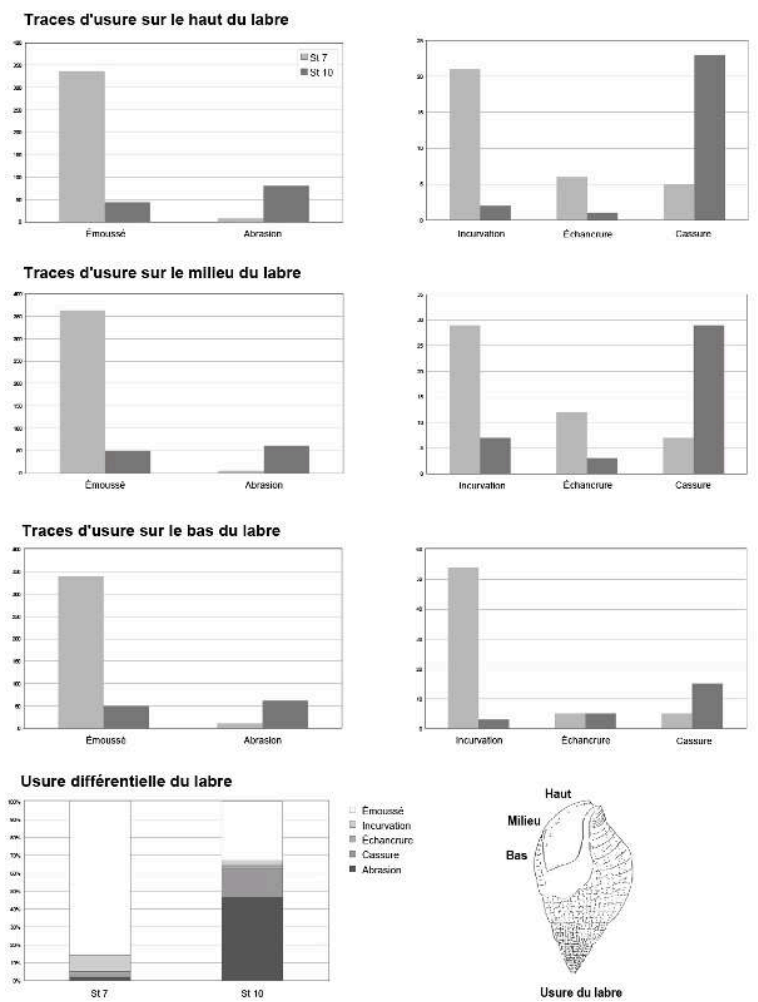

DAO L. Laporte ; dessin Y. Gruet. 
tels stigmates sont généralement situés en vis-à-vis de stigmates équivalents présents sur la lèvre de la perforation. Il s'agit sans doute là des témoins les plus fiables de l'usure laissés par un lien fin et étroit. C'est également la tension du lien qui pourrait être responsable de la cassure du test entre la perforation et le labre. Là, elle est le plus souvent localisée dans sa partie médiane ou dans une moindre mesure dans sa partie supérieure. Il est souvent difficile cependant de savoir le nombre de ces fractures qui ont eu lieu après le dépôt des objets dans la tombe, du fait de la fragilisation extrême de cette partie de la coquille. Quelques cas particuliers sont également intéressants à noter. Il s'agit notamment d'une coquille dont le labre cassé a été façonné par abrasion. Ensuite la perforation a été réalisée en E3-4, faute de place en E2 (fig. 35 nº 8).

L'usure du bord columellaire peut se traduire par différents degrés, du lustré à l'abrasion (fig. 37). Pour certains exemplaires, l'abrasion a provoqué la disparition totale $\mathrm{du}$ repli $\mathrm{du}$ bord columellaire. Une petite plage plane remplace cette zone convexe à l'origine. À proximité, le canal siphonal a subi des degrés d'usure variés, qui peuvent aller jusqu'à une déformation de sa forme d'origine. Cette zone plutôt aigüe sur les référentiels actuels collectés morts, présente une forme arrondie sur certains des exemplaires de La Vergne (fig. 38).

Fig. 37 - Usure du bord columellaire des nasses.

\section{Usure du bord columellaire}

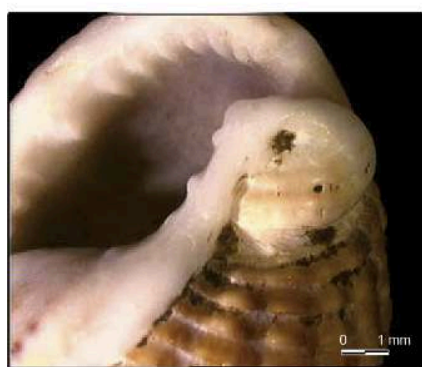

1. Coquille recueillie sur l'estran

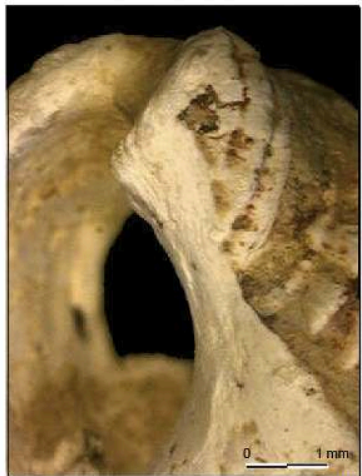

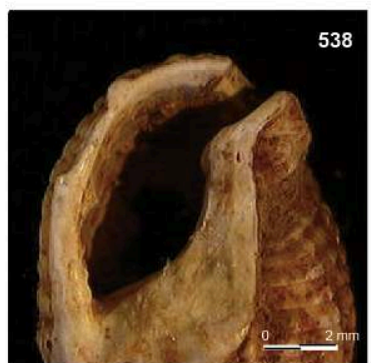

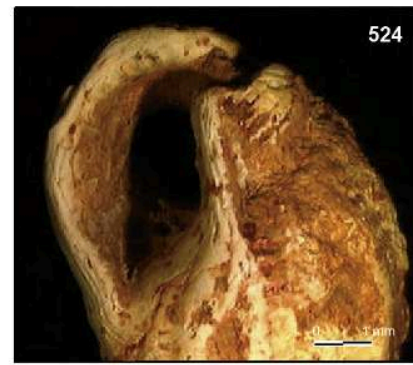

2. Aucune abrasion de la lèvre externe du bord columellaire
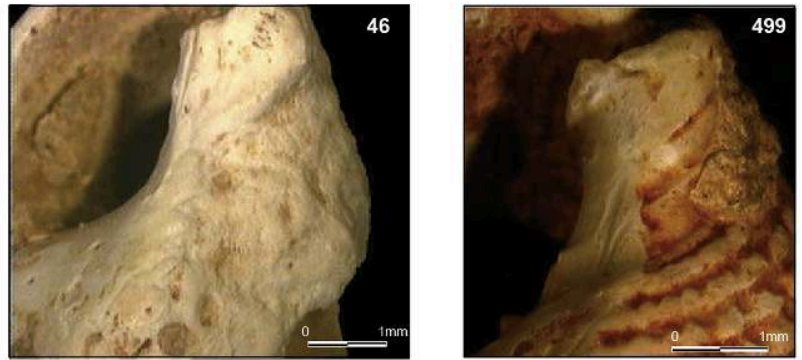

3. Forte abrasion de la lèvre externe du bord columellaire

Clichés L. Laporte et J.-Y Lefeuvre. 
Fig. 38 - Abrasion du canal siphonal des nasses.

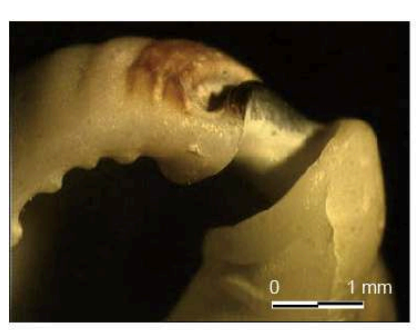

1. Coquille recueillie sur l'estran

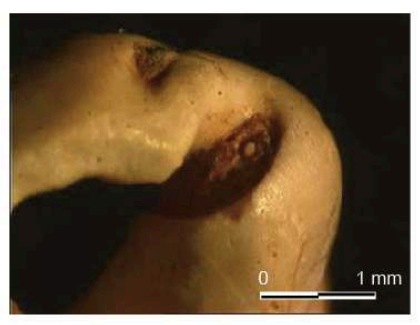

2. Abrasion du canal siphonal

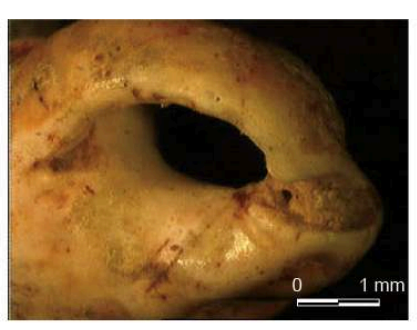

3. Abrasion du canal siphonal et du haut du bord columellaire

Clichés L. Laporte et J.-Y Lefeuvre.

L'axe d'allongement de la perforation marque une nette prédominance dans la direction 4-8, plus rarement 1-5 ou 3-7, presque jamais selon l'axe 2-6 (fig. 39). Nous avons vu que cela pouvait être lié en partie au mode de façonnage, en particulier lorsque la préparation de la perforation est obtenue par rainurage. L'incision est alors préférentiellement effectuée dans l'axe 4-8. Mais cet allongement préférentiel de la perforation est surtout lié aux traces d'usure observées sur la lèvre de la perforation. Le bord de cette dernière présente un profil arrondi ou incurvé vers l'intérieur dans presque tous les cas en 7-8-1, alors qu'il a tendance à être aplati vers l'extérieur en 3-4-5 (fig. 40). De ce point de vue les deux courbes de fréquence présentent des tendances exactement inverses. Les traces d'usures se répartissent également de la même façon. Les profils émoussés, pouvant conduire jusqu'à une incurvation du tracé de la lèvre, sont mieux représentés en 7-8, alors que les bords écaillés ou cassés sont plus fréquents en 2-3-4-5 (fig. 41). Chacun de ces deux différents types d'usure peut aboutir à une échancrure du tracé de la lèvre qui sera toujours nettement accentuée sur deux bords opposés de la perforation en 4 et en 8 . Un peu comme si l'usure du lien agissait de façon différenciée sur chacun de ces deux bords opposés. Quelques pièces présentent en effet une perforation échancrée à chacune de ces deux extrémités, ce qui lui confère une forme bilobée (fig. 42). Il existe même deux cas particuliers où la perforation a été déformée dans trois directions différentes.

Fig. 39 - Déformation de la perforation sur les nasses.
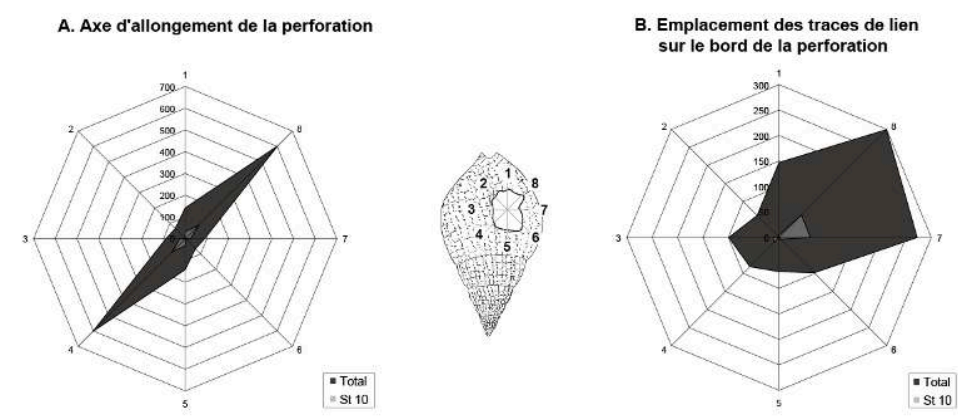

A. Axe d'allongement de la perforation; B. Emplacement des traces de liens sur le bord de la perforation.

DAO L. Laporte. 
Fig. 40 - Répartition des types de profils et des traces d'usure sur le bord de la perforation des nasses.
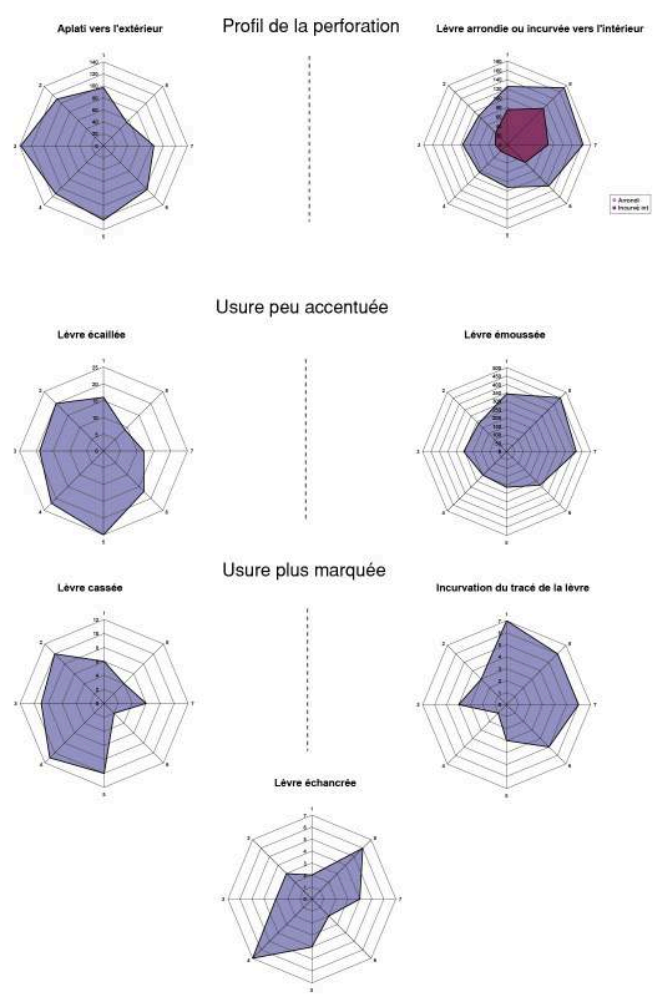

DAO L. Laporte.

Fig. 41 - Usure de la lèvre de la perforation sur les nasses.

\section{Usure de la lèvre de la perforation}
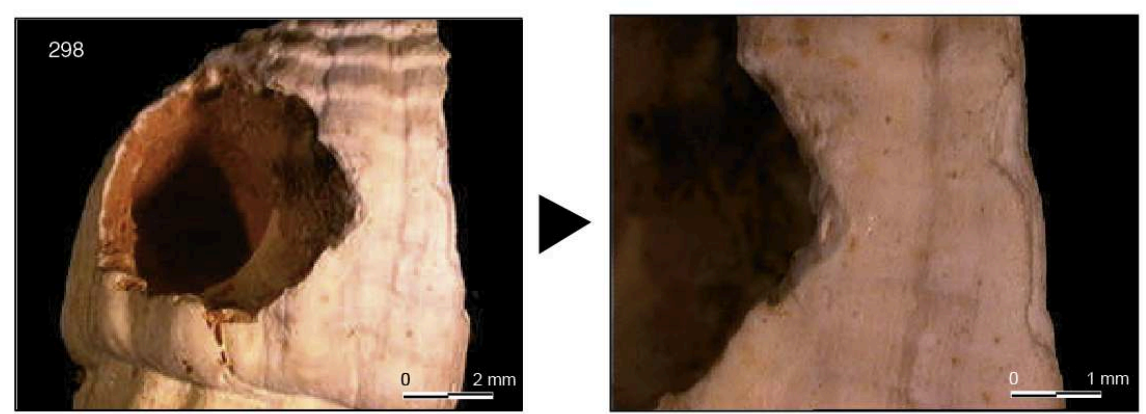

1. Écaillures
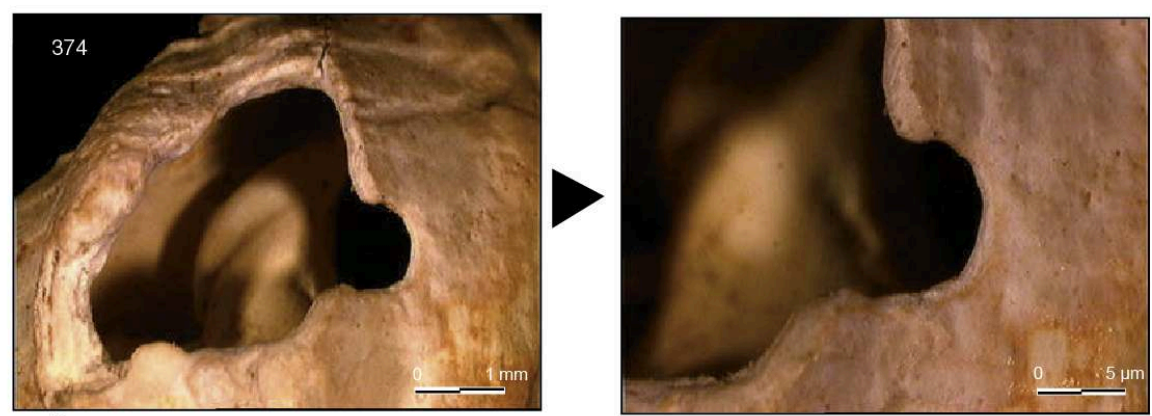

2. Échancrure du tracé de la lèvre

Clichés L. Laporte et J.-Y Lefeuvre. 
Fig. 42 - Échancrure de la perforation.

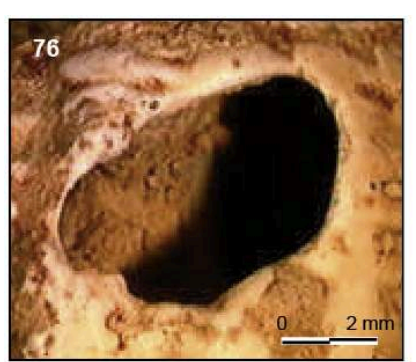

1. Émoussé de la lèvre en 7

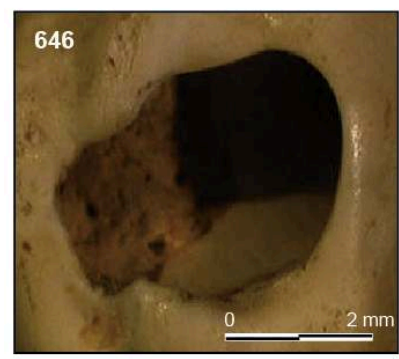

4. Forme bilobée

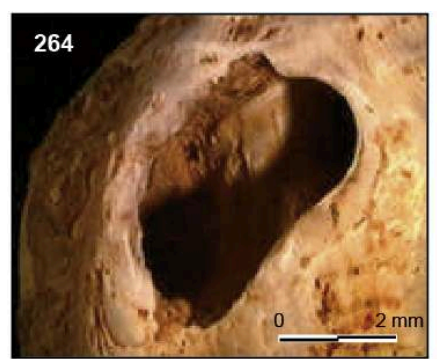

2. Lobe peu marqué en 7

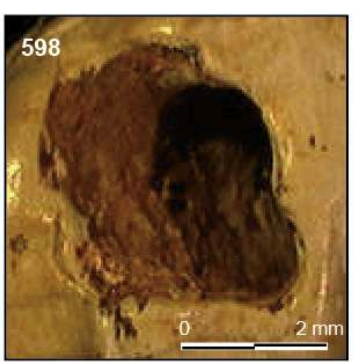

5. Lobe en 6

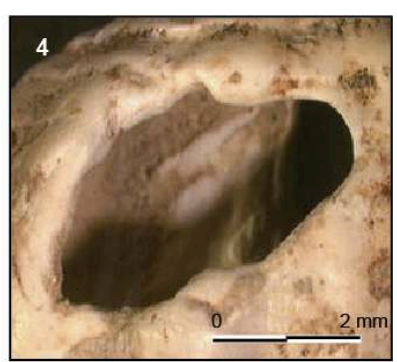

3. Lobe très marqué en 7

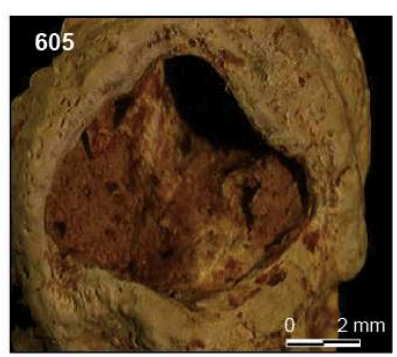

6. Au moins deux traces d'usures

Clichés L. Laporte et J.-Y Lefeuvre.

91 La présence de traces d'usure sur la lèvre de la perforation en 7 et 8 s'explique aisément, nous l'avons vu, par le passage d'un lien qui entre par l'ouverture naturelle de la coquille et ressort à cet endroit de la perforation pour faire le tour du labre. Quelques pièces présentent d'ailleurs une exacte correspondance, sur la face interne de la coquille, entre l'usure localisée des dents du labre et une abrasion de la face interne de la perforation en 8 . La présence d'un émoussé sur une lèvre de la perforation au profil arrondi ou incurvé vers l'intérieur est cohérente avec cette explication. Il est cependant impossible d'expliquer ainsi la présence de traces d'usure sur le cadran 3-4, opposé au précédent. Ces traces d'usure sont beaucoup plus rares que les précédentes, mais elles se retrouvent parfois sur les mêmes coquilles. La précédente hypothèse ne permet pas non plus d'expliquer la présence relativement fréquente d'une plage d'abrasion sur la partie supérieure du bord columellaire. Nous avons alors été amenés à proposer l'hypothèse de l'existence de deux liens, l'un fixant les coquilles sur un support en matière périssable qui fait le tour du labre, et l'autre reliant les coquilles entre elles, qui frotte contre le bord columellaire et passe par l'ouverture naturelle de la coquille pour ressortir par la perforation dans le cadran 3-4 (fig. 43). 
Fig. 43 - Des stigmates observés sur les coquilles aux hypothèses formulées quant à leur mode de fixation.

A
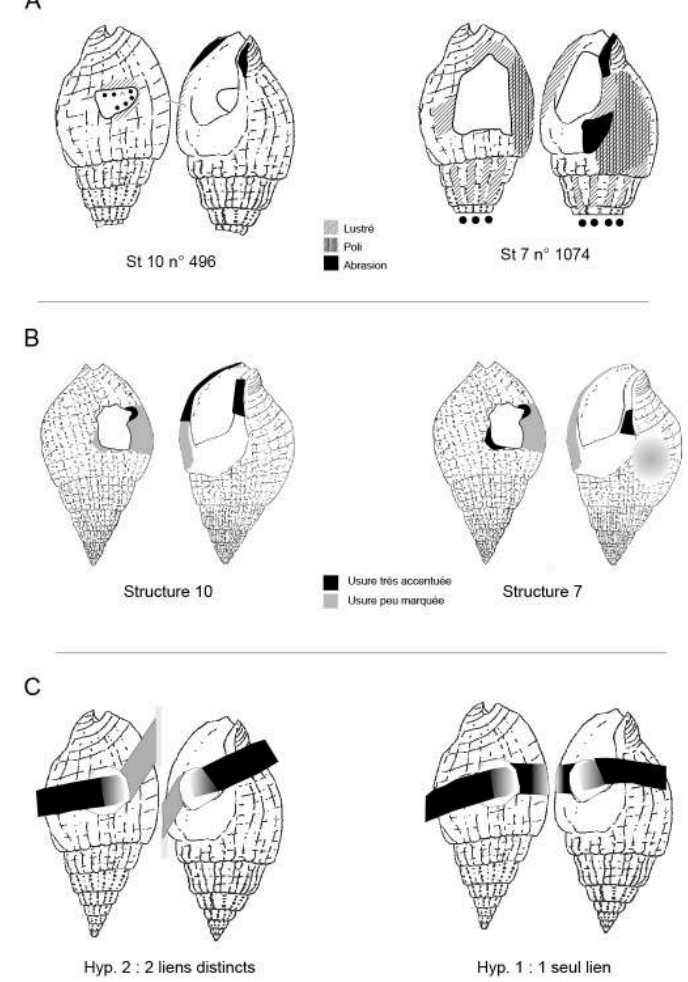

DAO L. Laporte ; dessins de la coquille Y. Gruet.

On peut alors expliquer l'usure différenciée des deux extrémités de la perforation, ainsi que son profil aplati vers l'extérieur dans le cadran 3-4. Précisons cependant que de telles considérations sur le mode de suspension ou de fixation des coquilles, élaborées à partir des données statistiques, ne constituent qu'une première approche forcément trop globalisante; première approche qu'il conviendra par la suite de confronter lot par lot aux observations faites sur le terrain.

L'usure du bord columellaire est en effet très différenciée sur les lots de coquilles provenant des structures 10, 7 et 3. Cette usure est proportionnellement beaucoup plus fréquente sur les lots issus des structures 10 et 3 , où elle est présente respectivement sur plus de $25 \%$ à $50 \%$ des pièces étudiées, alors qu'elle n'a été identifiée que sur $7 \%$ des pièces observées provenant de la structure 7. Dans les deux premiers cas, seule la partie supérieure est la plus affectée, notamment par une forte abrasion. En revanche dans la structure 7, l'ensemble du bord columellaire présente des traces d'usure (fig. 44). La nature et la position des traces d'usure sur le labre sont également très différenciées sur les nasses provenant des structures 7 et 10. Les traces d'usure localisées sont plus fréquentes dans le second cas où elles sont également plus prononcées sur le haut et le milieu du labre (fig. 36). Là, elles ont souvent conduit à une cassure du test en E1. En revanche sur les exemplaires de la structure 7, ce type d'usure se limite généralement à une incurvation du tracé, préférentiellement localisée sur le bas du labre. De même, alors que le labre des coquilles de la structure 7 porte souvent un simple émoussé, celles de la structure 10 présentent une abrasion nettement marquée dans plus de la moitié des cas. Il n'est d'ailleurs pas certain que cela soit dû à l'action de simples frottements, tant l'enlèvement de matière est alors important. 
L'hypothèse d'un façonnage peut être également envisagée. Quant aux traces d'usure sur le test, on remarquera la présence beaucoup plus fréquente de plages d'abrasion en E5 sur les coquilles appartenant à la structure 7. L'axe d'allongement des perforations étudiées sur les coquilles de la structure 3 présente par ailleurs une variabilité un peu plus grande que celle décrite sur l'ensemble du corpus.

Fig. 44 - Usure différentielle du labre sur les nasses provenant des structures 7 et 10.
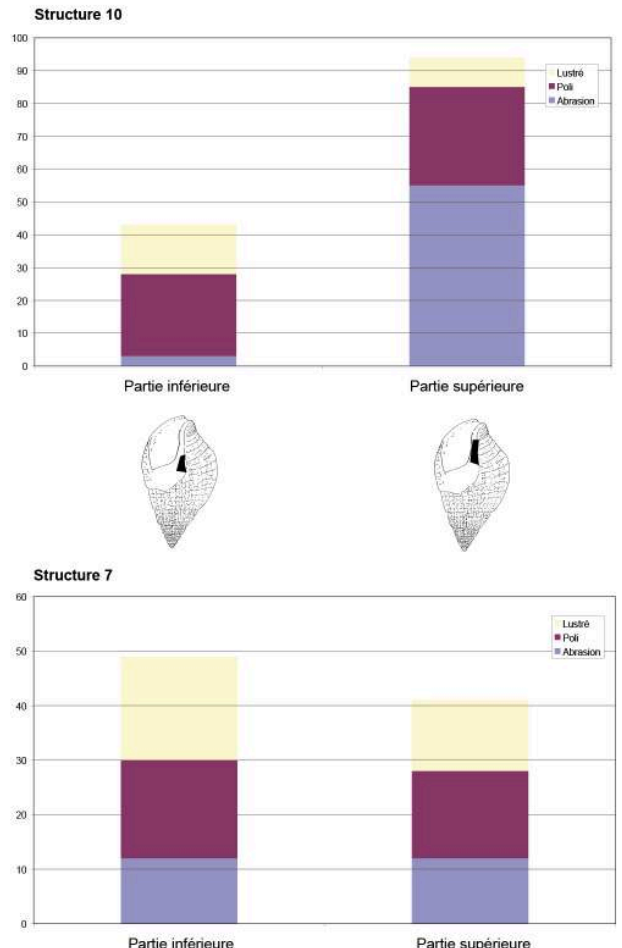

DAO L. Laporte ; dessin Y. Gruet.

\section{Nasses Tritia incrassata}

Cette espèce est présente dans deux structures funéraires. 7 exemplaires ont été trouvés dans la structure 10 et 26 dans la structure 7. Elle appartient au même genre que Tritia reticulata et se distingue de celle-ci principalement par une taille inférieure; taille qui n'est toutefois pas différente de celle des individus les plus petits de l'espèce Tritia reticulata parmi ceux utilisés sur le site (fig. 45). Toutes les perforations sont situées en E2 à l'exception de 2 cas isolés (l'une en E1, l'autre en E3-E4). 
Fig. 45 - A. Comparaison de la taille des Tritia reticulata $(N R=2107)$ et $T$. incrassata $(N R=38)$; B. Comparaison des Tritia sp. par structure : St. $3, N R=227$; St. 7, NR $=1534 ;$ St. 10, NR $=384$.
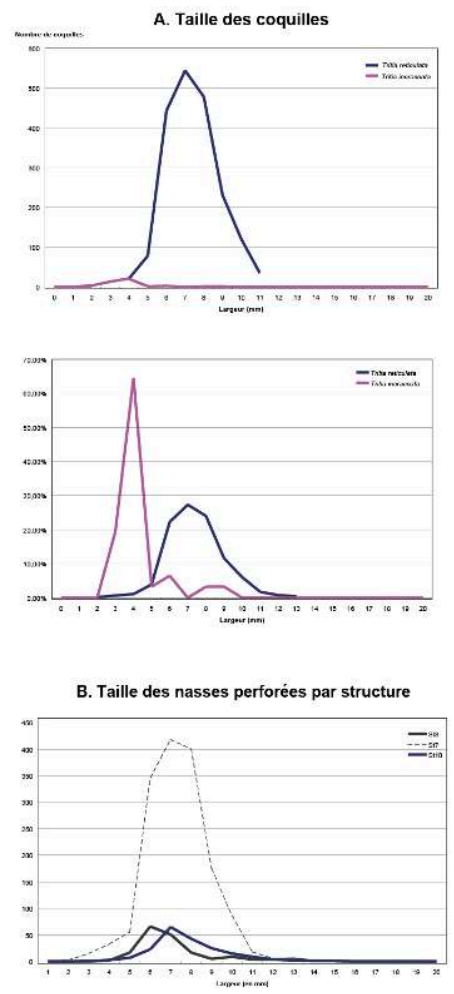

Réalisation de la figure L. Laporte et C. Dupont.

Les techniques de perforation: Elles sont là encore, difficiles à déceler. Les stigmates liés au port de la coquille ayant recoupé ou effacé les indices liés au percement. Seuls 3 exemplaires dont 1 de la St. 10 et 2 de la St. 7 présentent une surface plane sur le pourtour de la perforation. Ces stigmates peuvent correspondre à une préparation de la perforation par abrasion qui a parfois pu être menée jusqu'au percement.

Traces d'usure : L'étirement de la perforation a surtout été observé selon un axe 4-8. La forme de la perforation semble étroitement liée au port de la coquille, les traces d'usures étant préférentiellement situées en 7 ou (et) 8. Elles se traduisent par des échancrures du tracé de la lèvre, observées en 4 et en 8 , par une incurvation du bord interne de la lèvre de la perforation en 7 et 8 , et par une usure du profil externe en 4 . Outre la perforation, d'autres zones témoignent du passage du lien. C'est le cas du labre où les stigmates d'usure sont aussi bien représentés par une abrasion de la totalité du labre que par une usure préférentielle de la partie supérieure du bord interne du labre. De même, la columelle présente selon les cas des abrasions de la partie inférieure (1 cas) ou supérieure (4 cas). La localisation des stigmates d'usure sur les coquilles de Tritia incrassata est très proche de celle observée sur les coquilles de Tritia reticulata. On peut véritablement se demander si une quelconque différence a été faite entre les deux espèces, aussi bien lors de la collecte que dans leur utilisation.

\section{Natices Euspira catena}

Les natices proviennent de la structure funéraire 3 et du fossé 1 qui la recoupe (fig. 46). Elles présentent à chaque fois un nombre équivalent de 7 à 8 coquilles. Le lot de la structure 1 est plus altéré, mais on y retrouve des stigmates identiques. Dans tous les 
cas, l'unique perforation est située en E1, dans la partie inférieure du dernier tour de spire. Une seule coquille fait exception avec deux perforations côte à côte en E1 (fig. 47 $\mathrm{n}^{\circ} 2$ ). L'une d'entre elles est de même dimension que les précédentes. La technique mise en œuvre pour sa réalisation est également similaire. L'autre perforation est beaucoup plus grande, mais elle semble aussi avoir subi une légère usure du profil de la lèvre. Cette dernière perforation pourrait être une perforation naturelle reprise pour l'occasion. Une autre coquille présente une perforation naturelle (non utilisée) du dernier tour de spire en E5.

Fig. 46 - Euspira percée.

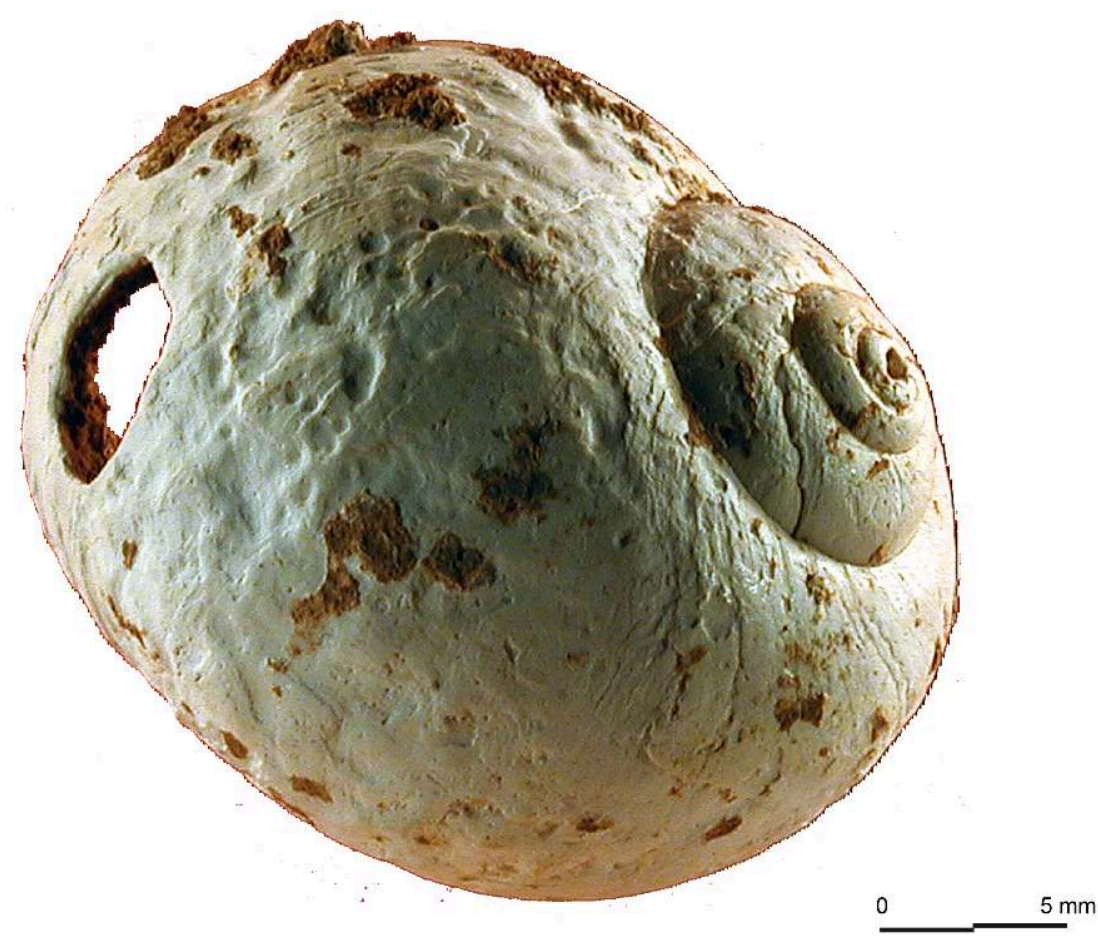

Cliché J.-Y. Lefeuvre. 
Fig. 47 - Techniques de perforation et traces d'usure sur les natices.

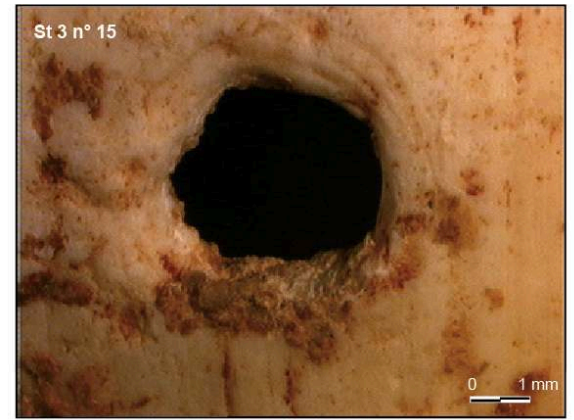

1. Stries concentriques résultant de l'utilisation d'un foret rotatif

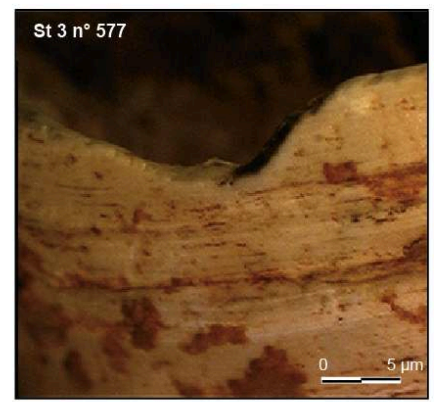

3. Échancrure dans la partie médiance du labre

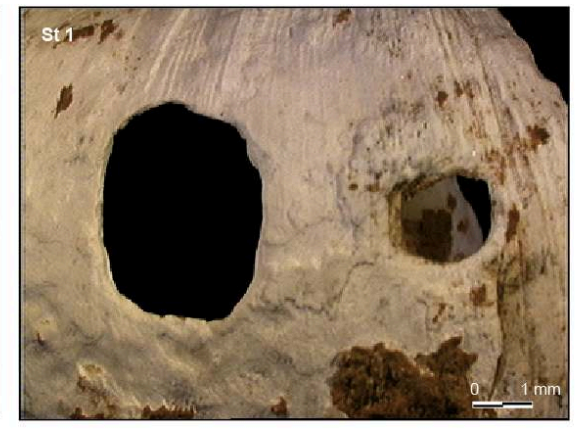

2. Deux orifices adjacents. Celui de droite a été obtenu à l'aide d'un foret rotatif

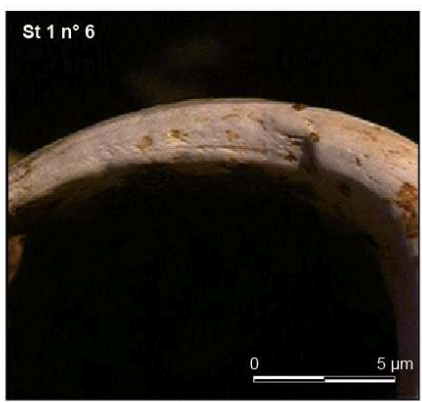

4. Échancrure du labre

Clichés L. Laporte et J.-Y Lefeuvre. l'action d'un foret rotatif qui a laissé des traces très nettes sur le pourtour de la perforation (fig. $47 \mathrm{n}^{\circ} 1$ ). Parfois cette action a pu être poussée jusqu'à la perforation. Mais, peut-être du fait de la fragilité de la coquille, il semble que dans la plupart des cas la perforation elle-même a été obtenue soit par percussion, soit, et c'est le cas le plus probable par la rupture de la coquille du fait de la pression exercée par la mèche du foret; la lumière de la perforation n'est donc pas toujours parfaitement circulaire, même si sa partie supérieure présente un profil conique bien caractéristique.

Les traces d'usures sont surtout présentes sur le labre (fig. $47 \mathrm{n}^{\circ}$ 3-4). Elles sont plus facilement visibles dans le lot provenant de la structure 3. Des échancrures sont présentes assez systématiquement en 3 points du labre ; dans sa partie supérieure, près du canal siphonal, où elle résulte le plus souvent d'une action abrasive; dans sa partie inférieure, près du dernier tour de spire, avec un écaillage bien caractéristique ; dans la partie médiane du labre, là où l'échancrure est souvent la plus marquée, quelques millimètres en dessous de l'emplacement de la perforation. Quelques rares perforations présentent une légère échancrure du contour de la perforation en 6 ou en 8.

Il faut ainsi insister sur le caractère homologue de ces deux lots qui pourraient avoir appartenu à un même ensemble sépulcral. Dans les deux cas les coquilles pourraient avoir été fixées sur un lien torsadé, comme cela a été proposé pour les coquilles de Tritia sp.; un brin vertical reliant les coquilles les unes aux autres aurait produit les échancrures supérieures et inférieures du labre, alors qu'un lien torsadé autour du premier, passant par la perforation et le milieu du labre, fixait la coquille sur le collier. Cette disposition expliquerait d'ailleurs également le léger décalage qui existe entre la position de la perforation et celle de l'échancrure correspondante sur le bord médian 
du labre. La présence d'un dentale dans l'une de ces coquilles, pourrait indiquer que la parure ainsi obtenue aurait alterné dentales enfilés sur le lien et Euspira sp.

\section{Nérites des rivières Theodoxus fluviatilis}

101 Seuls deux exemplaires de cette espèce ont été découverts dans la structure funéraire 3. Dans les deux cas la perforation principale est située en E2. Pour l'un des exemplaires, l'observation reste difficile, l'intégralité du contour de la perforation étant altérée. Pour le deuxième exemplaire, à la perforation principale s'ajoute une autre perforation, plus petite, sans doute liée à l'altération située en E3 (fig. 48). La technique de perforation reste difficile à définir du fait de l'observation d'un unique exemplaire. Des incisions sont cependant observées en 3 et en 7. Une incision du contour de la perforation présente en 1 est sans doute liée au port. Celle-ci est accompagnée d'un émoussé de la lèvre. La partie gauche du labre présente un écaillage de sa lèvre sans doute dû au recoupement de la structure de la coquille par l'usure du lien.

Fig. 48 - Techniques de perforation et traces d'usure sur les Theodoxus fluviatilis.

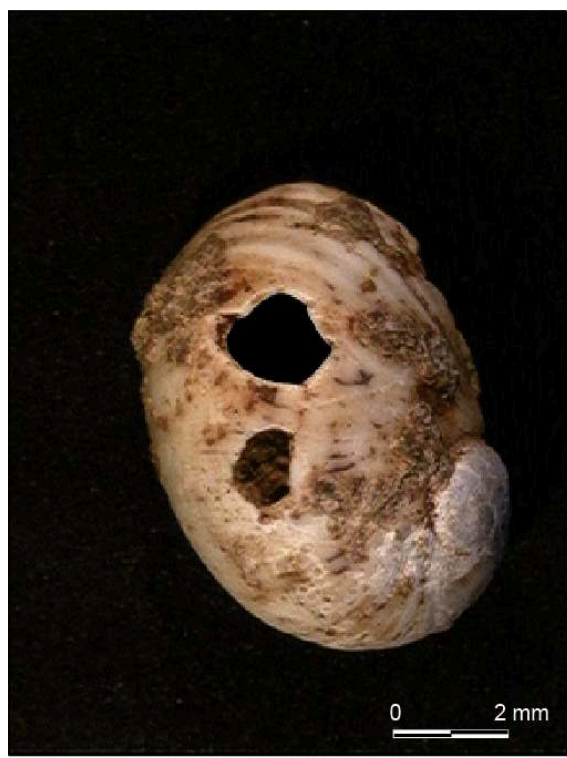

1. Theodoxus fluviatilis perforée

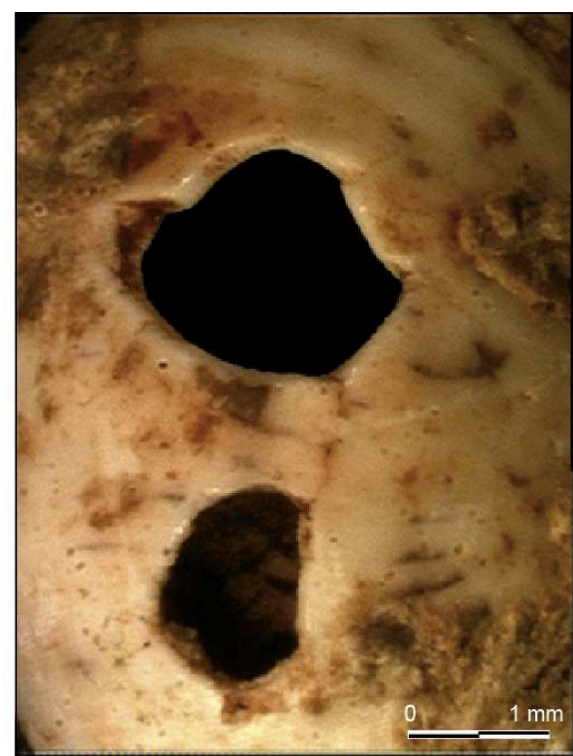

2. Détail des perforations. Noter l'échancrure de la perforation en E2

Clichés L. Laporte et J.-Y Lefeuvre.

\section{Murex Ocenebra erinaceus}

Une seule coquille de murex percée a été recueillie, au sein de la structure 7. L'apex du gastéropode est cassé et la surface du test très altérée. La perforation de forme quadrangulaire a été obtenue selon une technique précédemment décrite en ce qui concerne les nasses : deux incisions parallèles sont pratiquées à l'emplacement de la perforation en E1. Elles en délimiteront le contour. La perforation proprement dite est ensuite obtenue par percussion. 


\section{Patelles Patella sp.}

Les patelles ne sont représentées que dans 3 ensembles funéraires, toujours en petite quantité (3 exemplaires dans la structure 1, 2 exemplaires dans la structure 7 et 6 exemplaires dans la structure 10). Elles présentent toutes au moins une perforation, naturelle ou artificielle (fig. 49). Certaines portent également des stigmates liés à une utilisation prolongée.

Fig. 49 - Patelle percée.

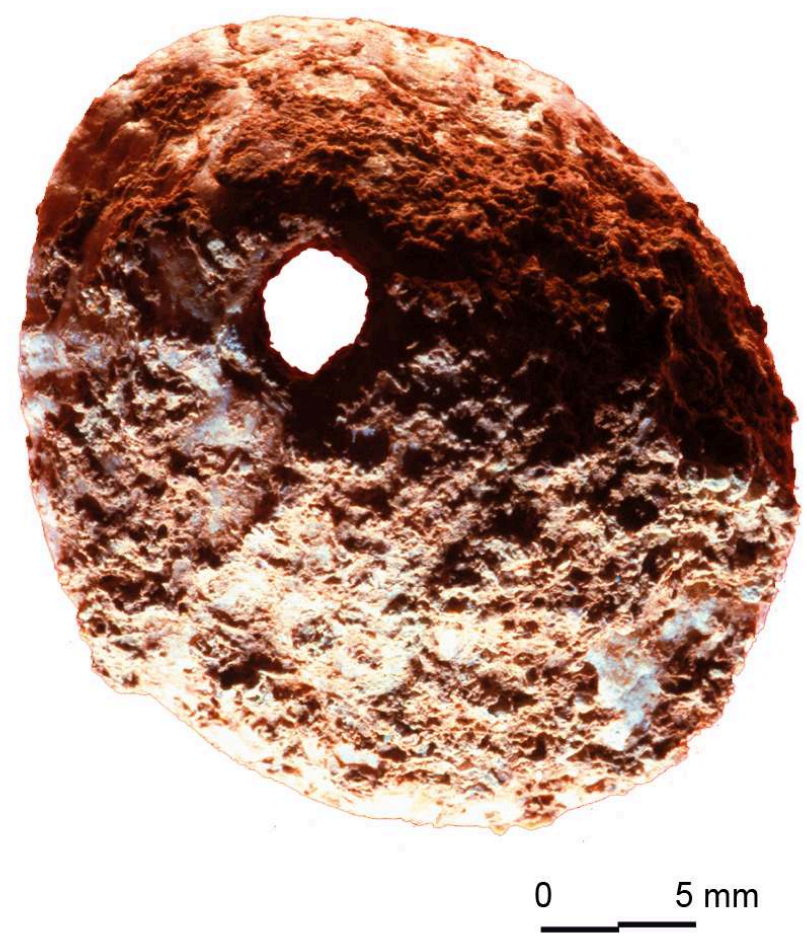

Cliché J.-Y. Lefeuvre.

La perforation est située principalement sur l'apex, mais parfois aussi sur l'un des bords de la coquille ( 2 exemplaires - fig. $50 \mathrm{n}^{\circ} 1$ ). Une seule patelle, provenant de la structure 7, présente deux perforations; l'une située sur l'apex et l'autre en partie cassée près du bord de la coquille. De nombreuses patelles échouées présentent naturellement une large perforation au sommet du cône résultant d'une fracture de l'apex. Ici, il semble bien que toutes les perforations observées au niveau de l'apex soient liées à une action anthropique. Elles ont été obtenues par percussion, provoquant de petites écaillures sur les faces interne et externe de la lèvre de la perforation. En revanche, celles situées sur le bord latéral de la coquille présentent une forme circulaire et régulière qui pourrait être liée à l'usage d'un foret rotatif. 
Fig. 50 - Localisation de la perforation et stigmates d'usure chez Patella sp.

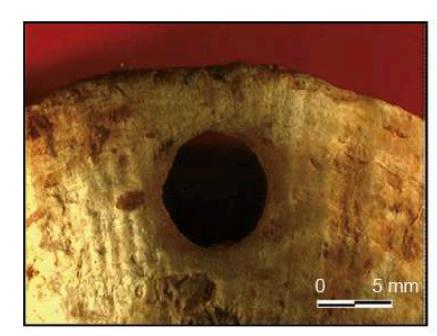

1. Perforation latérale

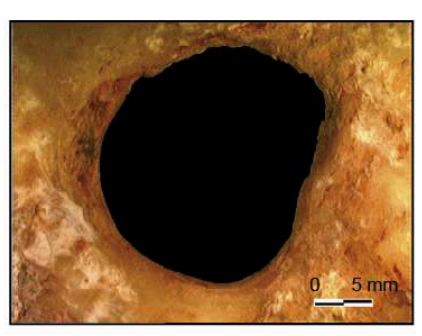

2. Déformation du tracé d'une perforation située à l'apex

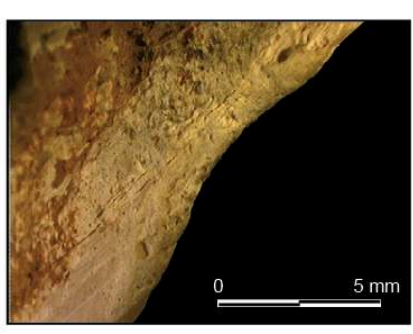

3. Abrasion du bord d'une patelle

Clichés L. Laporte et J.-Y Lefeuvre.

Des traces d'usure ont été observées sur 6 exemplaires. Lorsque la perforation est située sur le bord de la coquille de petites écaillures affectent le bord situé en vis-à-vis de la perforation. Ces traces d'usure pourraient être dues à la présence d'un lien reliant l'orifice de la perforation au bord de la coquille attachée à un support en matière périssable (fig. $50 \mathrm{n}^{\circ}$ 2). Lorsque l'usure est plus prononcée, ces petites écaillures forment une véritable encoche. De telles encoches sont toujours situées sur le bord correspondant à l'un des petits côtés de cette coquille de plan ovale, quand elle est vue du dessus. L'une de ces coquilles présente même deux encoches sur les bords opposés, de part et d'autre d'une perforation sommitale. Dans ce cas on observe également deux petites plages d'abrasion le long des bords allongés de la même coquille (fig. $50 \mathrm{n}^{\circ} 3$ ). On retrouve des plages d'abrasion similaires sur au moins 3 autres exemplaires. L'ensemble reste difficile à interpréter. Nous avons émis l'hypothèse que les écaillures et les encoches localisées pourraient être dues à la présence d'un liant passant par la perforation centrale et permettant de fixer la coquille sur un support en matière périssable. Les petites plages d'abrasion latérales, beaucoup plus larges, pourraient alors résulter du frottement des bords de la coquille sur un tel support, comme une lanière de cuir.

\section{Casque Semicassis saburon}

Deux coquilles de Semicassis saburon proviennent de la structure 10. Elles portent chacune deux perforations situées en vis-à-vis sur le dos de la coquille. La perforation la plus proche de l'apex est située dans la partie supérieure du cadran E2, alors que celle placée près de l'ouverture se trouve dans la partie inférieure du cadran E2 ou E3 (fig. 51). 


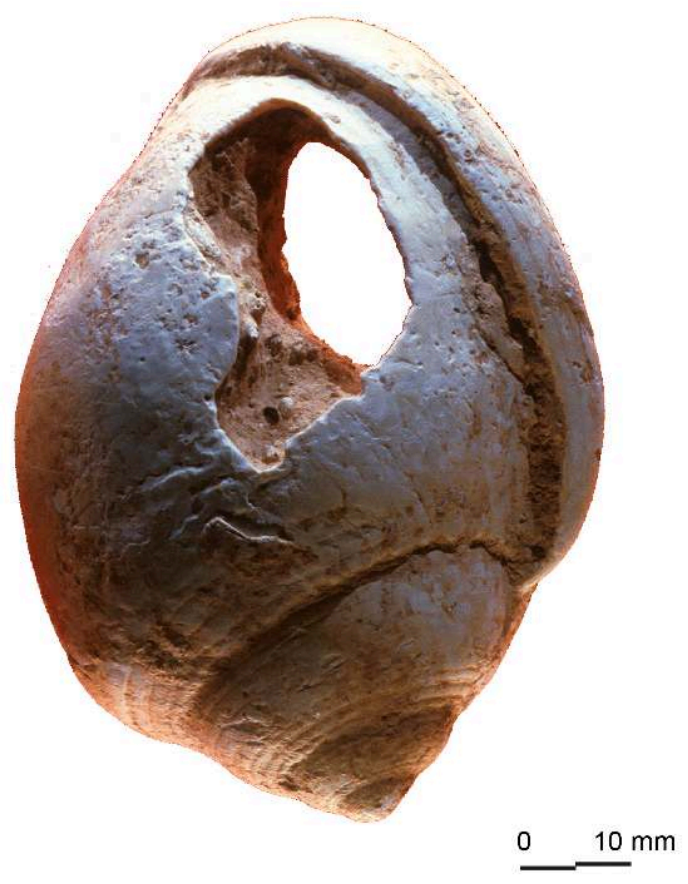

Cliché J.-Y. Lefeuvre.

107 L'une au moins de ces perforations a été réalisée intentionnellement: deux séries de petites encoches situées en vis-à-vis sur le bord de la perforation marquent encore la réalisation d'incisions parallèles visant à délimiter le contour de la future perforation. L'une des coquilles présente cependant, dans le cadran $\mathrm{E} 5$, une troisième perforation naturelle qui ne semble pas avoir été utilisée.

Dans les deux cas, les perforations situées près de l'apex présentent des traces d'usure. Il s'agit dans un cas d'une incurvation du tracé et dans l'autre d'une véritable échancrure au profil largement émoussé. Cette déformation du contour de la perforation est orientée vers l'apex qui, dans les deux cas, présente également des traces d'abrasion pouvant aller jusqu'à l'usure du canal siphonal. Nous avons interprété ces stigmates comme la marque d'un lien permettant de fixer la coquille sur un support, au niveau de l'apex et de l'ouverture. Frottant contre l'apex, le lien suivrait la courbe de la coquille pour rentrer à l'intérieur par la perforation adjacente. Il ressortirait au niveau de la seconde perforation. La tension du lien entre les deux perforations, et la tension qu'il exerce depuis l'intérieur de la coquille sur le test, pourrait d'ailleurs être au moins en partie responsable de la cassure qui relie ces deux perforations sur l'un des exemplaires (fig. 52). 
Fig. 52 - Traces d'usure sur le bord des perforations et le canal siphonal (Semicassis saburon).

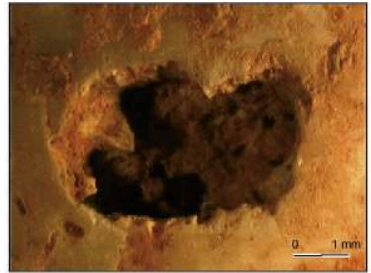

1. Perforation naturelle

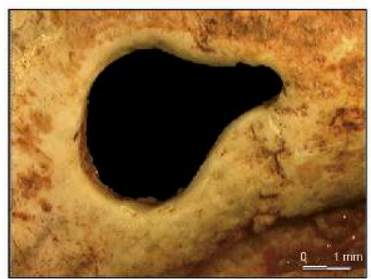

3. Échancrure du tracé de la perforation

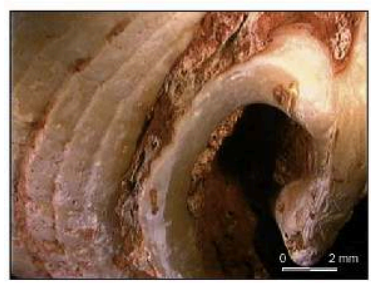

5. Usure du canal siphonal

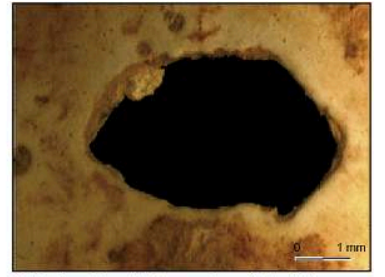

2. Perforation situee sur la partie inferieure

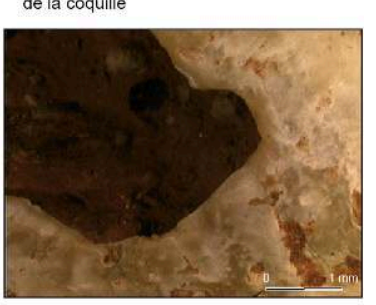

4. Incurvation du tracé de la perforation

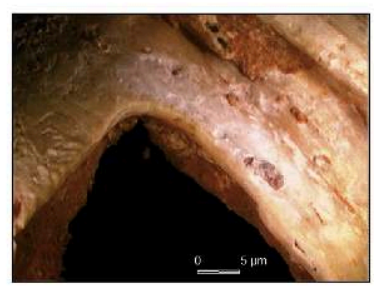

6. Détail de l'usure du canal siphonal

Clichés L. Laporte et J.-Y Lefeuvre.

\section{Bivalves}

109 Tous ces coquillages présentent une taille et une morphologie somme toute assez similaires. Les coquilles de Glycymeris glycymeris et de Laevicardium crassum ont d'ailleurs été utilisées semble-t-il indifféremment pour la confection d'un seul et même type d'objet de parure. Il s'agit de lots de 2 ou 3 coquilles emboîtées provenant principalement de la structure 10. Une coquille plus grande que les autres, contient alors sur sa face ventrale une ou deux autres coquilles disposées de façon similaire et de taille inférieure. Deux lots correspondent à deux coquilles emboîtées dont les charnières sont décalées à $90^{\circ}$ (438) ou opposées (428). Trois autres lots sont composés de 3 coquilles emboittées dont les charnières sont situées sur le même axe $(538,567)$ ou décalées (537; fig. 53-54). 
Fig. 53 - Valves trouvées emboîtées les unes dans les autres.

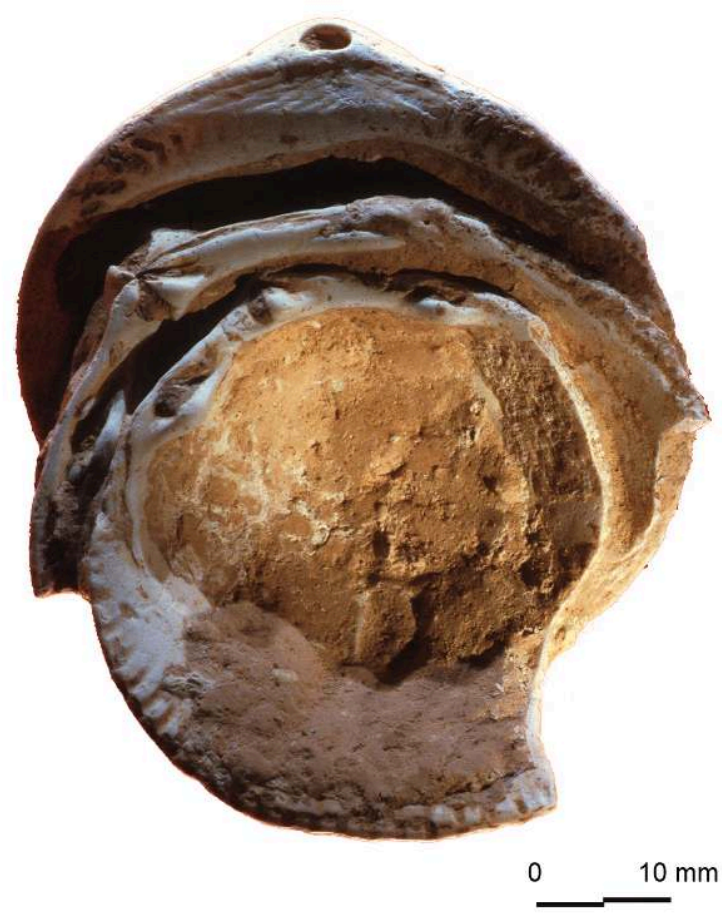

De haut en bas les espèces sont : Glycymeris glycymeris, Laevicardium crassum, Laevicardium crassum. Cliché J.-Y. Lefeuvre.

Fig. 54 - Valves de Glycymeris et de Laevicardium emboîtées.

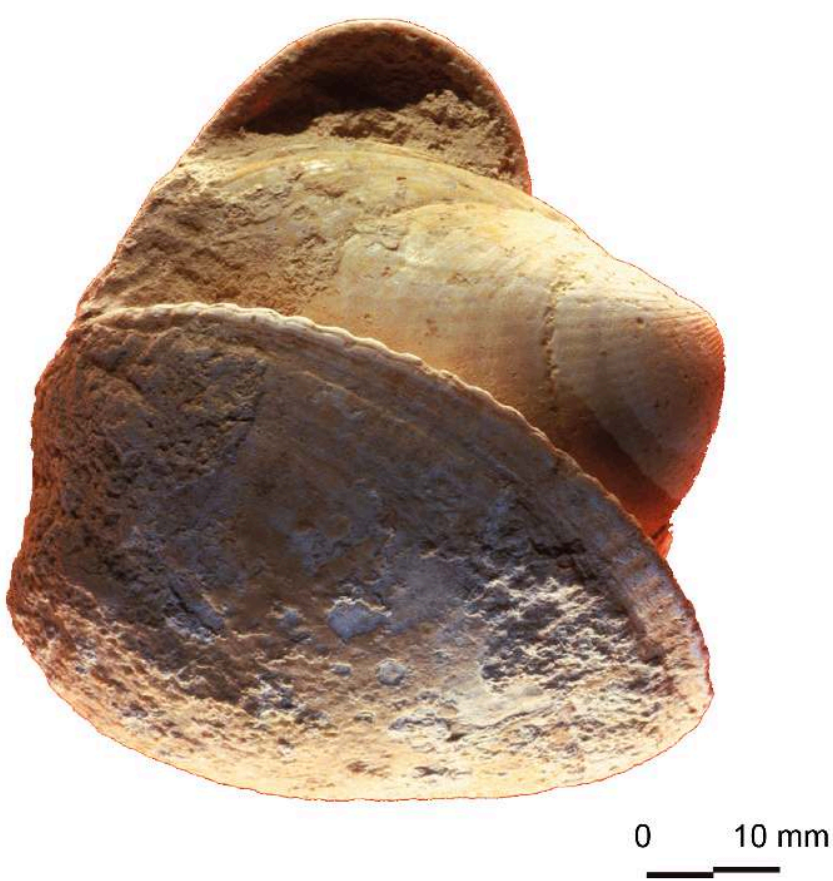

Cliché J.-Y. Lefeuvre 
110 Un dernier lot provenant de la structure 7 comporte trois coquilles emboîtées dont les perforations sont alignées. Dans ce dernier cas il s'agit de deux valves (Laevicardium sp.) pour la plus grande et de Glycymeris glycymeris pour la plus petite, avec une coquille de gastéropode au centre (Euspira catena ;159; fig. 55). Dans un souci de préservation, ces lots cimentés par la terre d'origine n'ont été nettoyés qu'au minimum, de sorte que les coquilles restent solidaires. De ce fait, certaines informations concernant notamment la partie dorsale de ces valves n'ont pas pu être observées.

Fig. 55 - Valves de Laevicardium et de Glycymeris emboîtées avec une natice à l'intérieur.

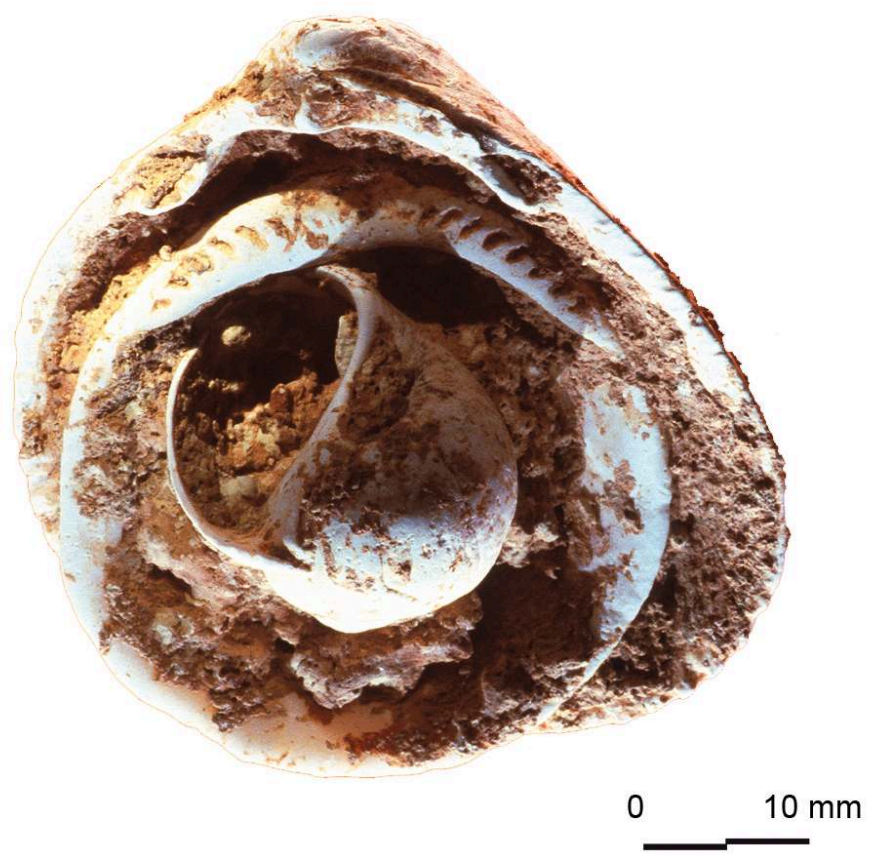

Cliché J.-Y. Lefeuvre.

111 Dans tous les cas, le crochet présente une perforation. Celle-ci se retrouve fréquemment à cet emplacement sur les coquilles ramassées en bord de mer (fig. 56 $\left.n^{\circ} 1-2\right)$. L'observation à la loupe binoculaire met en exergue la similitude entre les traces et les stigmates observés sur les coquilles de La Vergne et un échantillon de référence prélevé sur l'estran : on y retrouve une perforation dont le contour émoussé présente une forme géométrique, un peu irrégulière et dissymétrique, avec sur le bord de la perforation quelques écaillures localisées. Plus rarement elle présente une forme en entonnoir. Aucune de ces coquilles n'a révélé l'existence de perforations anthropiques, à l'exception toutefois d'une valve supérieure d'Ostrea edulis qui ne présente pas naturellement ce type de perforation et d'une valve de Glycymeris glycymeris (565) présentant une abrasion au niveau de son crochet (fig. $56 n^{\circ} 4$ ). Pour quelques cas isolés, la présence du sédiment et l'usure liés au port ayant pu masquer des stigmates de façonnage, il reste difficile de trancher entre une perforation d'origine naturelle ou anthropique. À cette exception près, la perforation présente sur le crochet de la valve résulte d'une action naturelle. L'unique action anthropique a consisté à 
sélectionner les coquilles percées qui seules convenaient à l'usage qu'il souhaitait en faire.

Le caractère émoussé du bord de la valve est également un critère qui permet d'affirmer que ces coquilles ont été ramassées mortes sur la plage. Certaines sont d'ailleurs cassées, et ces cassures présentent les mêmes polis que sur les coquilles que nous avons nous-même ramassées échouées. Sur l'une de ces coquilles, la cassure est recouverte d'ocre rouge, comme la face dorsale de la valve. Ceci indique que la cassure n'est pas liée à des phénomènes taphonomiques, mais bien que la coquille cassée ait été portée en l'état comme élément d'un objet de parure.

Fig. 56 - Emplacement et techniques de perforation sur les valves.

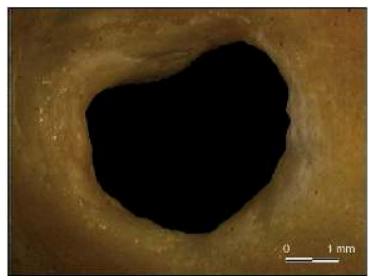

1. Perforation naturelle liee a Pusure marine

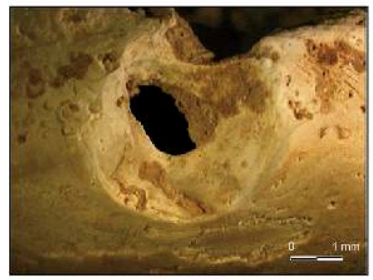

3. Perforation d'une spisule présentant

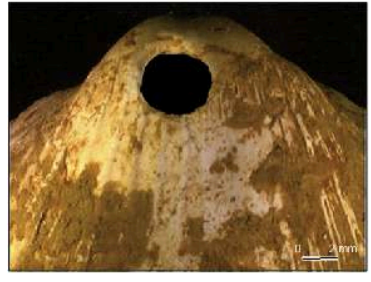

5. Perforation à lumbo de Cerastodermaedule

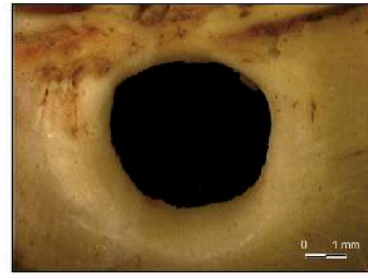

2. Perforation d'un bivalve prèsentant des stigmates proches de ceux d'une coquille èchouee

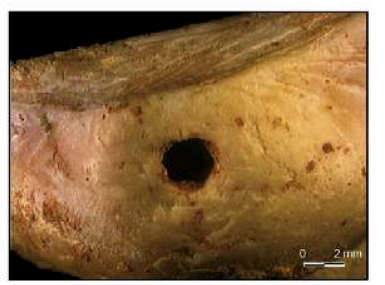

4. Perforation située au niveau du crochet, avec préparation par abrasion (Glycymeris)

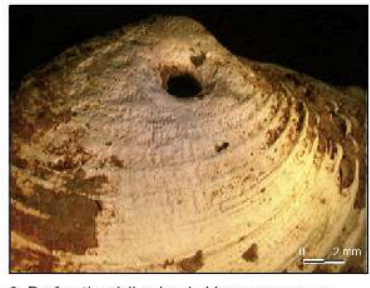

6. Perforation à lumbo de Venus verrucosa

Clichés L. Laporte et J.-Y Lefeuvre.

\section{Coques Cerastoderma edule}

113 Une unique valve de coque a été recueillie dans la structure 7. La perforation est située sur l'umbo. Elle présente un émoussé plus accentué dans un cadran en direction du crochet et légèrement décalé par rapport à ce dernier (fig. $56 \mathrm{n}^{\circ}$ 5).

\section{Amandes de mer Glycymeris glycymeris}

114 Trois valves percées de Glycymeris sp. proviennent de la structure 10. Deux autres valves percées proviennent respectivement des structures 1 et 2 . La perforation, située près $\mathrm{du}$ crochet est naturelle. Dans la majorité des cas elles sont directement associées à des coquilles de Laevicardium crassum avec lesquelles elles sont emboîtées. Aucune trace d'usure n'a été observée. Une petite coquille isolée de Glycymeris sp. présente également une usure sur la face ventrale de la coquille, en vis-à-vis de la perforation dans la partie 
qui sépare les dents de la charnière (fig. $57 \mathrm{n}^{\circ} 2-3$ ). Cette usure a provoqué une nette incurvation du tracé du bord interne de la charnière. Elle a été interprétée comme celle d'un lien de suspension.

Fig. 57 - Traces d'usure sur les valves.

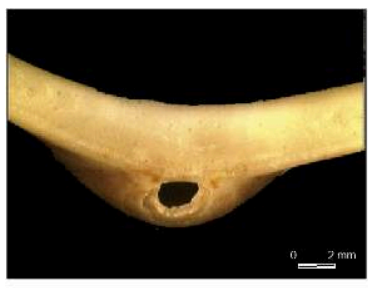

1. Charnière d'une coquille collectée sur estran

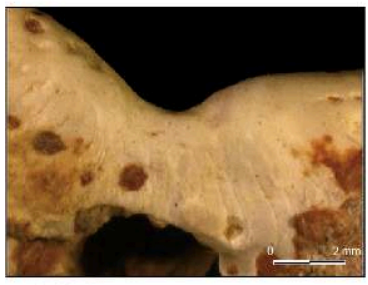

3. Détail de l'usure

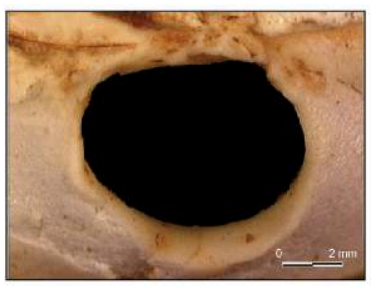

5. Usure du profil de la perforation

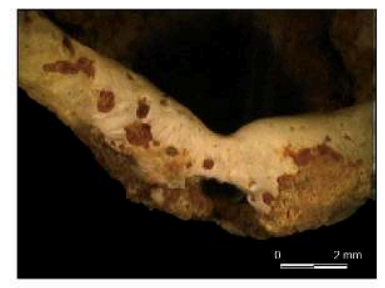

2. Charnière d'une coquille de La Vergne présentant une usure

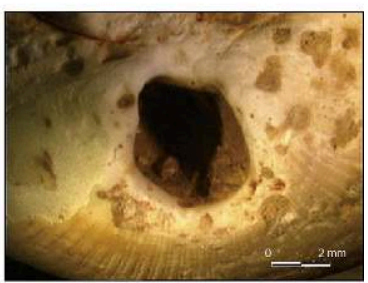

4. Échancrure de la perforation sans doute liée au port de la coquille

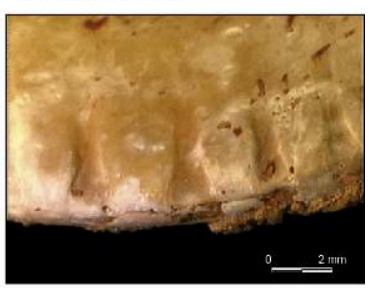

6. Émoussée du bord de la valve

1. Glycymeris glycymeris ; 2-6. Laevicardium crassum

Clichés L. Laporte et J.-Y Lefeuvre.

\section{Bucardes de Norvège Laevicardium crassum}

Elles sont surtout représentées dans la structure 10 où on en compte 12 exemplaires. Deux autres valves percées proviennent respectivement des structures 1 et 7 . La perforation située près $d u$ crochet est naturelle. Certaines valves ont été recueillies individuellement à la fouille, d'autres ont été prélevées ensemble. On peut parfois observer quelques traces d'usure sur le bord de la perforation ( 5 exemplaires dont 438, 428, 437 de la structure 10, 159 de la structure 7 et une dernière de structure 1). Elles sont marquées par un émoussé plus appuyé de la lèvre de la perforation dans sa partie qui débouche sur la face dorsale (fig. $57 \mathrm{n}^{\circ} 4$ ). Il peut s'agir d'une usure qui résulte du frottement d'un lien. Dans le cas des lots de deux ou trois valves encastrées, il pourrait alors s'agir d'un lien permettant le maintien et la suspension de chaque pièce au sein d'une même parure.

\section{Huîtres plates Ostrea edulis}

116 Un seul fragment de valve supérieure d'huître percée a été recueilli dans ces ensembles funéraires (fig. 58). Il provient de la structure 10. La perforation est largement décentrée. Elle se trouve à l'une des extrémités de la valve. Sur une partie de sa 
circonférence, la lèvre de la perforation présente de nombreuses écaillures qui suggèrent une perforation obtenue simplement par percussion. L'autre moitié de la circonférence présente un contour régulier et très émoussé. Le tracé de la lèvre dessine ainsi une échancrure décentrée vers le bord adjacent de la coquille. Nous avons interprété ce type de stigmate comme une marque d'usure liée au passage d'un lien dans la perforation. Dans ce cas précis, l'emplacement de la perforation et des traces d'usure sur cette dernière, comme l'absence de traces d'usure sur le bord correspondant de la coquille, suggèrent une suspension de la pièce par l'intermédiaire d'un lien.

Fig. 58 - Valve droite d'huître percée.

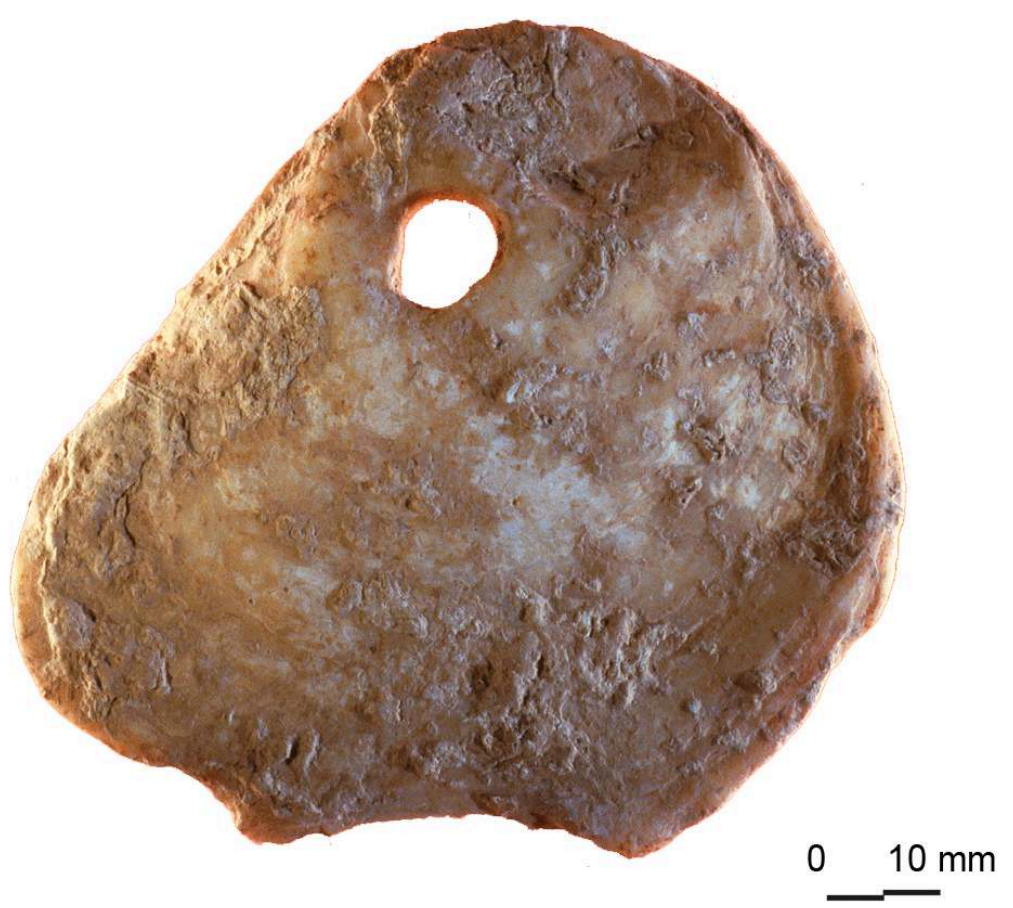

Cliché J.-Y. Lefeuvre

Spisule Spisula sp.

Deux spisules percées proviennent de la structure 10. Comme dans les cas précédents, la perforation est située au niveau du crochet. Sa forme en entonnoir et son emplacement correspondent aux traces laissées par une espèce de perforant qui s'attaque notamment à ce coquillage. Les seules traces d'usure qui pourraient être interprétées comme liées à l'usage qui a été fait de ces objets, consistent en deux petites encoches opposées sur le bord de la valve de l'un des deux spisules (510) recueillies dans cette structure. L'une de ces encoches est marquée par quelques écaillures, alors que l'autre, un peu plus large, est matérialisée par une incurvation du tracé du bord de la coquille. Cette incurvation est liée à une abrasion localisée du bord de la coquille ; abrasion qui elle-même recoupe l'émoussé naturel de ces coquilles recueillies sur l'estran. Aucune autre trace d'usure n'a été observée sur la partie dorsale, ventrale, ou sur le bord des valves étudiées (fig. 56 n³). 


\section{Praire Venus verrucosa}

118 Un seul exemplaire est représenté qui provient de la structure 1. La perforation, très altérée, est située près de la charnière. Aucune trace d'usure n'a pu être observée (fig. $56 \mathrm{n}^{\circ}$ 6).

\section{Traces d'usures et modes de suspension : comparaison avec des collections ethnographiques}

119 Certains dentales, souvent décrits comme enfilés le long de liens (Rivière 1905, BargeMahieu 1991), ont effectivement été trouvés à La Vergne emboîtés les uns dans les autres. Ils peuvent alors avoir constitué les éléments de colliers. D'autres en revanche, semblent avoir été cousus sur un support en matière périssable.

Sur les valves de bivalve, la lèvre de la perforation porte assez fréquemment les traces d'une usure parfois prononcée qui atteste d'une utilisation prolongée de la parure. Les valves étaient suspendues par un lien qui parfois également maintenait plusieurs coquilles emboîtées les unes dans les autres. Ce dernier cas concerne plus de la moitié des valves percées recueillies au cours de la fouille.

La plupart des coquilles de gastéropodes observées portent des traces d'usure, et ce quelle que soit l'espèce concernée. Nous l'avons dit, cette première approche statistique offre une vue sans doute assez réductrice de la complexité et de la diversité des modes de suspension mis en œuvre. Cependant, les traces observées ne sont guère compatibles avec des coquilles simplement enfilées sur un lien pour former un collier. La grande majorité d'entre elles semblent avoir été fixées sur un support en matière périssable, sans préjuger pour autant, ni de la nature de ce dernier, ni de l'agencement des pièces entre elles. Le mode de fixation est toutefois différent suivant les espèces. La majorité des Tritia sp. semblent avoir été fixées à l'aide de deux liens aux fonctions distinctes, ou d'un lien torsadé à deux brins, qui passaient par l'ouverture de la coquille pour ressortir au niveau de l'unique perforation. Le passage du lien est donc transversal par rapport à l'axe de la coquille. Des traces d'usures similaires ont été observées sur les coquilles d'Euspira sp. Les deux grosses coquilles biforées de Semicassis saburon semblent en revanche avoir été fixées par un lien unique, qui rentre par une perforation pour ressortir par la seconde, dans l'axe du plus grand allongement de la coquille. Quant aux patelles, les traces d'usure observées seraient également compatibles avec une fixation sur un support long et étroit comme une lanière de cuir par exemple (fig. 59). 
Fig. 59 - Récapitulatif de l'emplacement, de la fréquence et de l'intensité des traces d'usure sur le test.
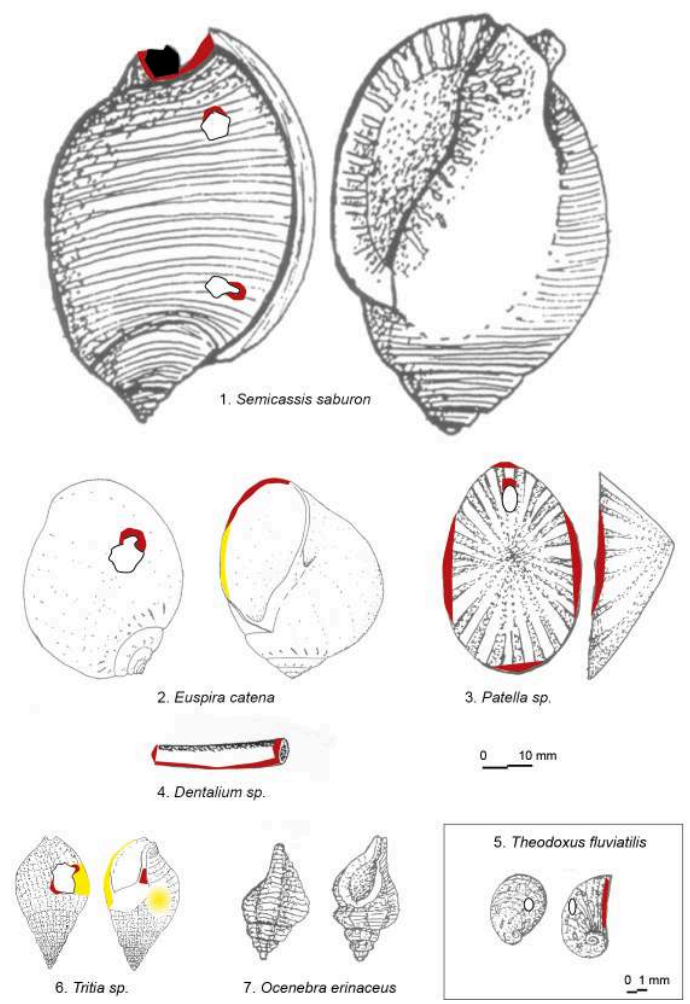

ROUgE : Traces fréquentes et généralement bien marquées ; JAUNE : Traces généralement plus ténues et parfois moins marquées.

DAO L. Laporte; dessins des coquilles Y. Gruet et C. Dupont.

À l'occasion d'une mission en Argentine, nous avons eu la surprise d'observer dans une vitrine du musée ethnographique de La Plata et celles du Musée Municipal d'Olavarria des colliers de gastéropodes fixés selon un procédé similaire à celui que nous avions envisagé, de façon purement théorique, pour les nasses et les natices percées de La Vergne. Ces colliers étaient portés au siècle dernier par un groupe de chasseurs cueilleurs de Patagonie : les Yamanas (fig. 60). Les coquilles de gastéropodes uniforées sont reliées par un lien torsadé comprenant deux brins aux fonctions différentes. Un brin vertical relie les coquilles les unes aux autres, en passant par l'ouverture naturelle de la coquille. Un lien torsadé autour du précédent s'enroule autour du labre pour assurer la fixation de la coquille sur son support. Les traces d'usures sont similaires à celles que nous avons sur les nasses et les natices du site de La Vergne (fig. 61). En particulier, nous avons observé quelques écaillures, voire une véritable incurvation du tracé du labre sur quelques pièces. Parfois également, la perforation présente une déformation très nette dans le cadran 7-8. Il va de soi que toutes les pièces d'un même collier ne présentent pas le même degré d'usure, cette dernière résultant principalement de tensions très localisées. 
Fig. 60 - Collier de gastéropode yamana (Patagonie).

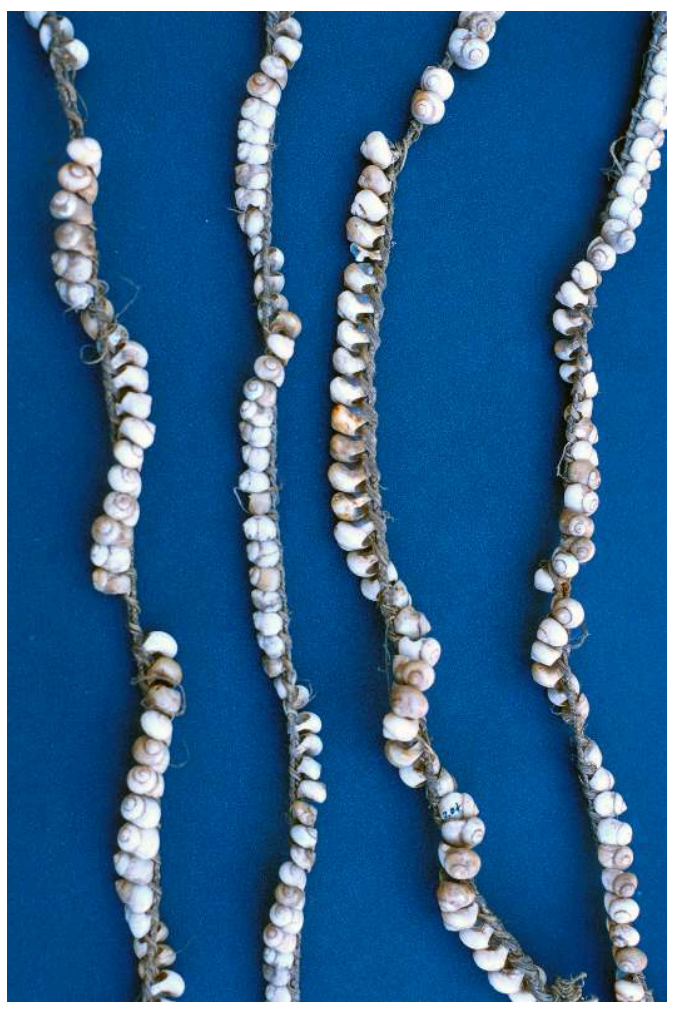

Coll. Musée municipal d'Olavarria, Province de Buenos Aires, Argentine ; cliché L. Laporte. 
Fig. 61 - Détail du mode de suspension sur le collier de gastéropode yamana (Patagonie). Remarquer la présence d'une échancrure du labre correspondant à l'emplacement du passage du lien.

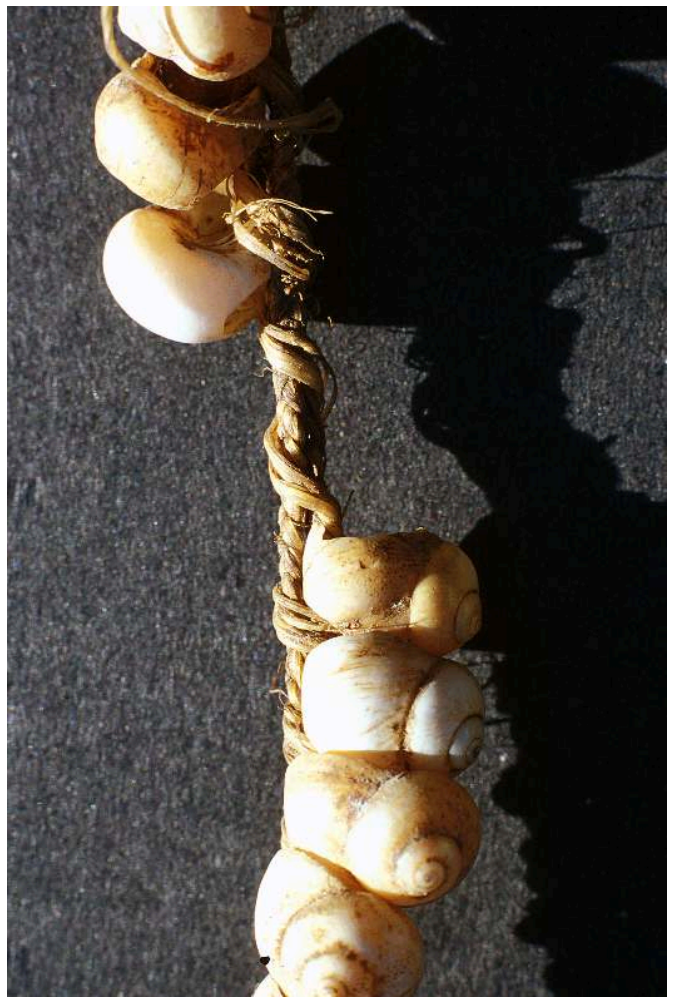

Coll. Musée municipal d'Olavarria, Province de Buenos Aires, Argentine ; cliché L. Laporte.

Cet exemple ethnographique n'est pas le fruit d'une enquête systématique. Il n'a donc pas valeur de preuve quant à l'hypothèse que nous formulons, mais il permet du moins de tester la validité d'un raisonnement élaboré précédemment sur la seule base d'une observation des pièces archéologiques.

\section{Répartition des pièces dans la tombe : une mise en scène des corps humains}

La nécropole de La Vergne, a livré l'un des plus importants lots de parures mésolithiques en France, et plus généralement en Europe Occidentale. Le terme même de parure suggère que ces objets étaient portés à titre d'ornements corporels, seuls ou fixés sur un vêtement. Nous verrons qu'il faut parfois se méfier d'une telle définition, d'abord parce que le costume ne saurait se réduire aux seuls objets recueillis en fouille, ensuite parce que certaines pièces percées peuvent tout aussi bien orner quelques objets indépendants du volume corporel.

125 À première vue, les coquilles percées sont si abondantes dans chacune de ces sépultures qu'elles apparaissent d'abord comme un semis de points presque continu dans tout le volume des fosses correspondantes. Pour les objets les plus volumineux, quelques concentrations apparaissent immédiatement, comme la présence de nombreuses valves autour de la tête de l'adulte inhumé dans la structure 10. Pour le reste, qui constitue l'essentiel du corpus, il semble bien difficile et aléatoire de vouloir reconstituer une quelconque organisation dans l'agencement de ces milliers de petites pièces. Cette 
même difficulté avait d'ailleurs été rencontrée lors de la fouille des sépultures mésolithiques de Téviec et Hoëdic. Marthe et Saint-Just Péquart indiquent que les squelettes de deux enfants en connexion déposés dans la sépulture $C$ de Hoëdic étaient « littéralement farcis de littorines percées, au point que le montant de la récolte que nous en faisons atteint 2900 pièces ». Pour autant «il est impossible d'observer en place le mobilier et les ornements en coquillage appartenant en propre à chaque défunt. Nous devons nous contenter d'en recueillir les nombreux éléments, essaimés un peu partout aux alentours et parmi les ossements » (Péquart et Péquart 1954, p. 35).

En ce qui concerne les sépultures de La Vergne, nous avons bénéficié d'une documentation riche et de qualité remarquable, surtout si l'on veut bien se rappeler qu'il s'agit là d'une intervention d'urgence. Les dessins effectués sur le terrain sont de très bonne qualité : chaque fois que cela était possible, les principales caractéristiques de la coquille ont été figurées avec précision (perforation, ornementation de la coquille, charnière et crochets pour les valves, ouverture, labre et siphon pour les gastéropodes...). Chaque pièce a été cotée, numérotée et décrite séparément. Les critères retenus par les fouilleurs sont pertinents (orientation de la coquille, face d'apparition, etc.). Seules les photos se sont parfois révélées d'une définition insuffisante, en particulier pour les espèces malacologiques les plus petites qui représentent l'essentiel du corpus.

Très naturellement, quand il s'agit d'une sépulture, la fouille est généralement guidée, techniquement et mentalement, par les volumes corporels des défunts. Une telle démarche est parfaitement adaptée à la reconstitution de la parure lorsque cette dernière est composée de bracelets, de colliers ou est fixée sur des pièces de vêtement que portait le défunt à l'occasion de son inhumation. Il peut arriver cependant que ces mêmes pièces participent également à la constitution ou l'ornementation d'objets déposés dans la tombe au côté du défunt. Elles présentent alors une organisation spatiale dont les volumes sont totalement indépendants du corps inhumé, même s'ils ont pu être localement influencés par les mouvements taphonomiques liés à sa décomposition. Pour peu que ces objets soient principalement constitués d'éléments en matière périssable, alors seules ces petites ornementations permettront d'en détecter la présence, à défaut de pouvoir en définir précisément la nature ou la fonction.

Distinguer ce qui ressort de la parure corporelle et ce qui appartient à tout autre chose sera notre première préoccupation. Les hypothèses que nous serons amenés à proposer sont fondées sur l'étude spatiale détaillée de critères biologiques, techniques et fonctionnels observés sur chacune de ces pièces, en tenant compte aussi de l'évolution taphonomique de l'ensemble de la sépulture. Ce travail a été effectué à l'aide du logiciel Adobe Illustrator en reportant sur autant de calques que nécessaire toutes les informations contenues par les relevés et les minutes de terrain. Cette méthode présente l'avantage de pouvoir contrôler simultanément la superposition des différentes pièces relevées (pierres, ossements, coquilles...) et leur position altimétrique respective. Lourde et fastidieuse, elle n'a été appliquée qu'aux trois structures les mieux conservées. Seuls ont été retenus ici les critères qui, parfois à notre grande surprise, se sont révélés pertinents quant à l'organisation des coquilles percées dans la sépulture. Pour autant, il conviendra de rester prudents dans l'interprétation des résultats, tant il est toujours délicat de restituer a posteriori une organisation des vestiges qui n'a pas été directement observée sur le terrain. Ce n'est que dans un second temps que nous serons amenés à discuter le statut des offrandes funéraires et 
de la parure corporelle associées à chaque individu, ou groupe d'individus. Dans cette démarche les observations des anthropologues seront stratégiques.

\section{La tombe 7}

Cette tombe contenait les restes de deux individus en connexion et ceux d'un enfant, ces derniers étant mêlés à quelques os brûlés. Elle contenait 1961 pièces en coquille. Une très grande majorité a été soigneusement dessinée à la main, pièce par pièce sur une quinzaine de relevés successifs, au fur et à mesure de l'avancement de la fouille sur le terrain. Quelques pièces, environ 150, ont été situées sur trois photographies verticales, prises successivement et constituant autant de relevés intermédiaires. Un nombre limité de pièces, enfin, a été prélevé par lots sans être situées individuellement, devant l'urgence de terminer cette fouille de sauvetage. Seules ces dernières ne pourront pas être prises en compte dans l'analyse que nous proposerons.

À la fouille, la plupart des objets en coquille semblaient répartis de façon assez aléatoire dans toute l'épaisseur du remplissage de la fosse. C'est effectivement l'image que nous avons également obtenue en projetant sur un même plan horizontal le dessin de toutes les pièces dessinées (fig. 62A). On note cependant sur cette même figure, un espace vide de mobilier à l'extrémité sud de la fosse, limité par un bois de cerf qui pourrait appartenir à la superstructure de la tombe. L'image n'est guère différente si l'on élimine les pièces reportées sur les relevés correspondant aux tout premiers décapages effectués, pour ne garder que ceux qui concernent la base du remplissage de la fosse (R4 à R17). Toutefois, on observe ici un effet de paroi assez net qui isole une zone de forte concentration de coquilles, au centre et au nord de la fosse. Au-delà, seules quelques rares pièces isolées annoncent l'espace vide de tout mobilier en coquille, au sud (fig. 62B). Cet effet de paroi correspond à un ressaut dans le creusement de la fosse sépulcrale. Il n'est vraiment visible que si l'on reporte sur un même relevé toutes les pièces situées à différents niveaux altimétriques : cet alignement ne correspond donc pas à une file continue de coquilles. Ces dernières, situées à différents niveaux sur un même plan vertical, pourraient correspondre à une enveloppe, sans doute en matière périssable, plaquée contre les parois de la fosse (pièces cousues sur une peau ou une natte en fibres végétales par exemple). Au sein de l'espace ainsi délimité, se trouvent notamment les corps des deux personnes inhumées en position fortement contractée. Il nous a ensuite paru utile de retirer les coquilles percées qui pouvaient être liées aux volumes corporels de ces deux défunts, en les distinguant de celles qui ne l'étaient assurément pas (fig. 62C). Deux lots distincts apparaissent ainsi nettement dans l'espace libre situé au pied des personnes inhumées. Ils avaient été d'ailleurs parfaitement identifiés par l'équipe sur le terrain. Au sein du premier lot, situé à l'est, on observe la présence de très nombreux dentales alors qu'ils sont pratiquement absents du deuxième lot (fig. 62D). Parallèlement, on observe que l'individu situé à l'est semble associé à un nombre de dentales bien plus important que le deuxième individu déposé contre son dos. Au terme de cette première analyse, une première organisation des vestiges dans la fosse sépulcrale apparaît donc assez clairement. 
Fig. 62 - Répartition des coquilles dans la structure 7.
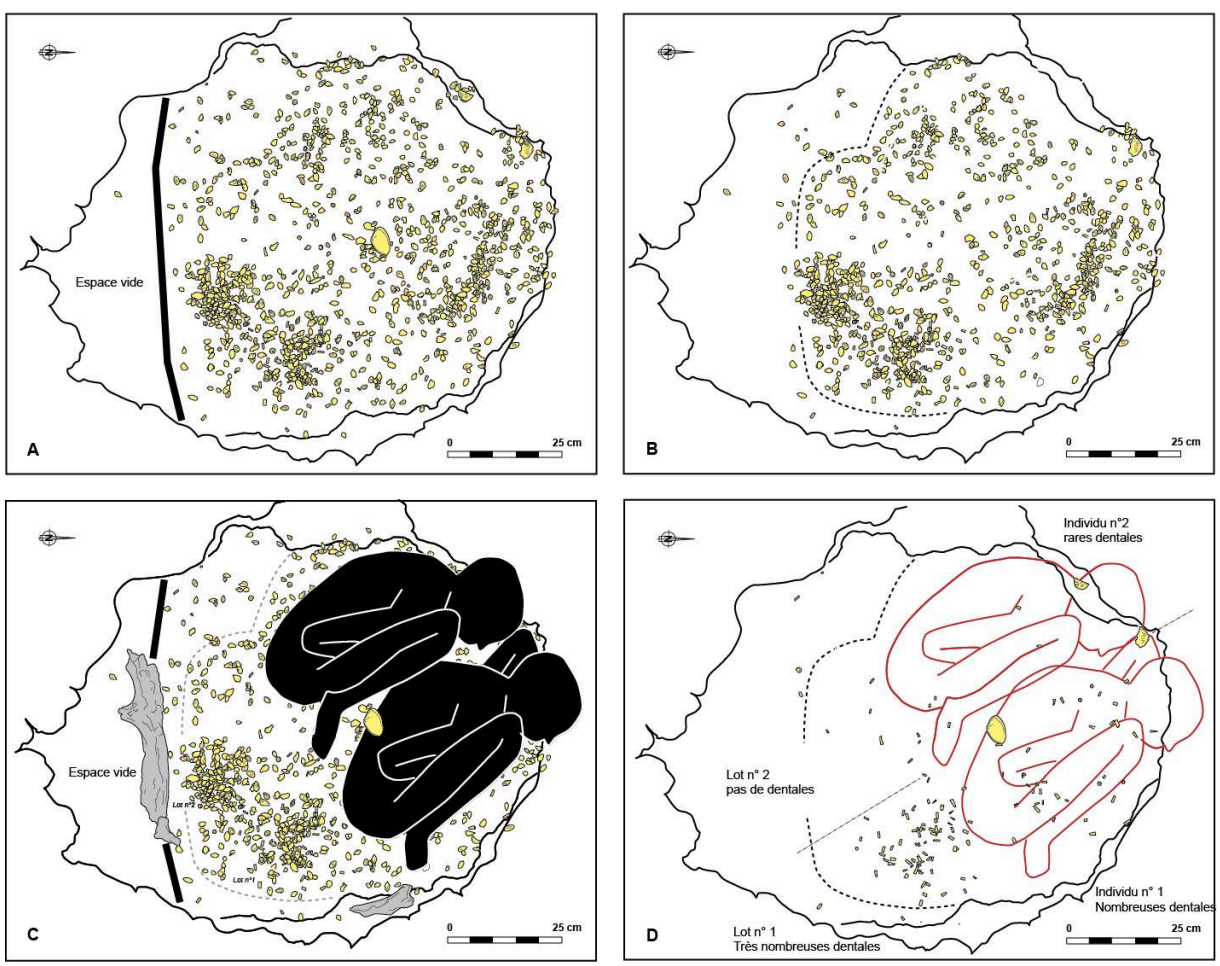

Construction de la figure par L. Laporte, DAO de L. Quesnel pour le dessin de chaque pièce indépendamment, telles que reportées sur les relevés de fouilles originaux dessinés par H. Duday avec également des annotations de P. Courtaud.

Nous nous sommes ensuite attachés à l'étude des coquilles percées qui ne semblaient pas directement liées aux volumes corporels des deux individus en connexion. La présence de ces pièces dans la fosse nous intriguait car elles ne pouvaient être assimilées à des objets de parure portés par les défunts. S'agissait-il de petites coquilles projetées à la volée au cours de la cérémonie, un peu comme autant de grains de riz à la sortie de l'un de nos mariages actuels ? En reportant sur un même plan toutes les pièces situées à la même altitude, l'organisation du dépôt apparaît peu à peu. Rares sont les pièces situées au-dessus de la cote $-50 \mathrm{~cm}$ (fig. 63A). Certaines sont très proches du corps des défunts. Quelques nasses suivent la courbe du dos de l'individu ${ }^{\circ} 2$. Elles sont souvent appariées et se superposent alors, avec l'apex situé dans la même direction et les perforations en vis-à-vis. Le caractère récurrent d'une telle disposition semble exclure que cet alignement soit lié au travail d'un fouisseur. Peut-être étaient-elles fixées sur un ruban de toile ou une lanière de cuir ? Cette file se termine par une grosse valve percée, située un peu au-dessus de la nuque de l'individu ${ }^{\circ} 2$. 
Fig. 63 - Coquilles disjointes des volumes corporels des deux individus en connexion de la structure 7.
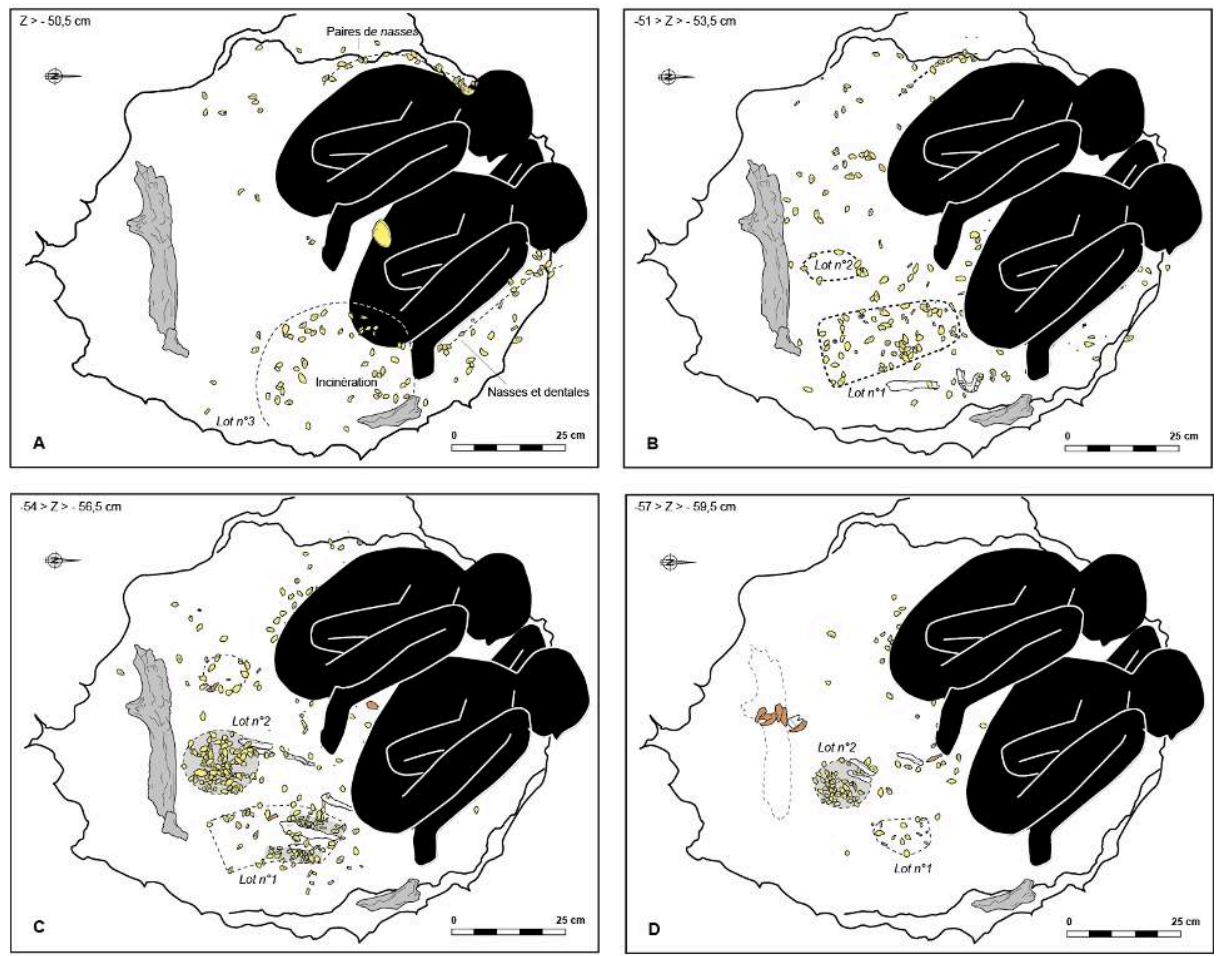

Construction de la figure par L. Laporte, DAO de L. Quesnel pour le dessin de chaque pièce indépendamment, telles que reportées sur les relevés de fouilles originaux dessinés par H. Duday avec également des annotations de P. Courtaud.

Un autre ensemble de nasses et de dentales se trouve au-dessus et le long de la jambe droite de l'individu $n^{\circ} 1$, sans toutefois que l'organisation des pièces soit aussi claire que dans le cas précédent. En revanche, l'espace central de la fosse est presque vide de coquilles percées. À l'est on observe une nouvelle concentration bien marquée. La plupart des coquilles sont situées entre les cotes $-45 \mathrm{~cm}$ et $-50 \mathrm{~cm}$. On s'aperçoit alors qu'elles dessinent une demi- couronne, large de 5 à $10 \mathrm{~cm}$ (fig. 64). Le retour aux relevés de terrain nous indique que cette couronne de pièces percées se superpose à un petit amas d'os brûlés correspondant aux restes d'un sujet incinéré. Les nasses percées sont généralement situées au-dessus, mais parfois aussi mêlées aux fragments d'os brûlés. Aucune d'entre elles ne porte toutefois de trace de combustion. Cette association, bien délimitée dans l'espace, a peu de chance d'être fortuite. À titre d'hypothèse, on peut imaginer que ces coquilles ornaient un objet en matière périssable (peaux, couverture, natte, etc.) qui recouvrait ou contenait les restes du sujet incinéré. Lors de la décomposition de cet objet, quelques pièces se seraient alors déplacées vers le bas de quelques centimètres. 
Fig. 64 - Le lot $n^{\circ} 3$ de la structure 7.

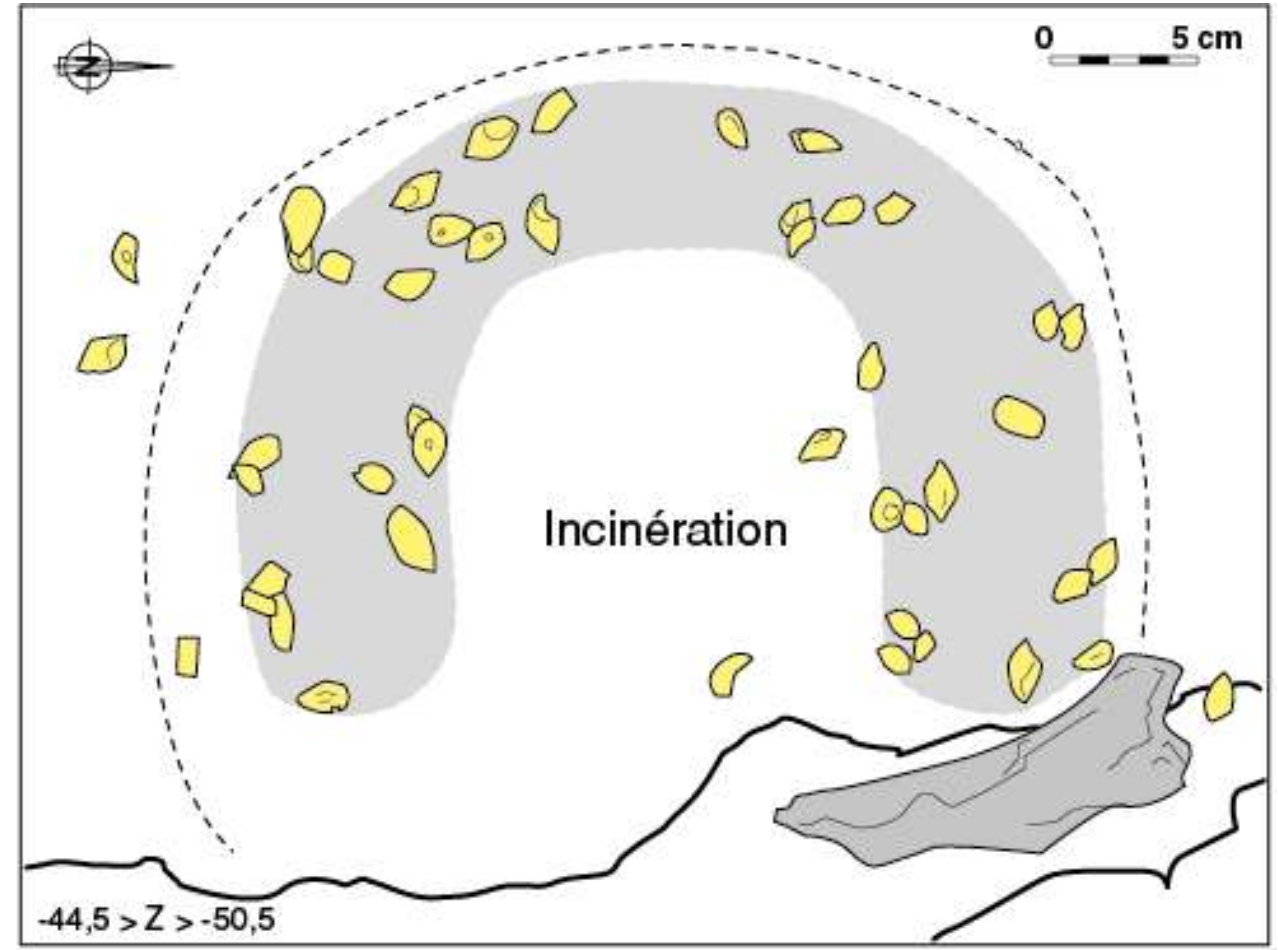

Construction de la figure par L. Laporte, DAO de L. Quesnel pour le dessin de chaque pièce indépendamment, telles que reportées sur les relevés de fouilles originaux dessinés par H. Duday avec également des annotations de P. Courtaud.

Les deux concentrations remarquées à la fouille n'apparaissent que dans les dix derniers centimètres du remplissage de la fosse. Sur le plan d'ensemble ces deux nébuleuses de points se recouvraient partiellement (fig. 62C). Si l'on cartographie les pièces pour chaque niveau altimétrique, elles constituent en revanche deux concentrations toujours nettement distinctes dont les centres de gravité se déplacent progressivement vers l'est au fur et à mesure que l'on descend dans le remplissage (fig. 63B, C, D). Le volume dans lequel s'inscrit le lot $n^{\circ} 1$ peut être assimilé à celui d'un manchon. Deux os d'un enfant et une dent animale percée sont étroitement mêlés à ces nombreuses coquilles percées. Ils sont donc contenus dans ce volume, plutôt vers sa base (fig. 65). Les nasses constituant le lot $\mathrm{n}^{\circ} 2$ dessinent tout d'abord une couronne, puis forment comme les différents brins d'une résille au fur et à mesure que l'on descend dans le remplissage (fig. 66A). Vers la base, deux os d'enfant et deux dents animales percées recouvrent ici encore quelques coquilles (fig. 65B). Dans ce cas, le volume décrit est plus proche de la sphère. À titre d'hypothèse, on peut envisager que les coquilles étaient placées dans un contenant, voire même ornaient initialement les brins d'un filet ou les parois d'un sac en matière périssable. C'est également ce que suggèrent les photos prises sur le terrain (fig. 66B). Il est remarquable que l'on retrouve dans les deux lots précédents une association symétrique avec une ou deux dents animales percées et deux os d'enfant. De ce point de vue l'étroite imbrication des os longs d'enfant à la base des deux concentrations de coquilles percées pose problème. Soit ces os d'enfant appartiennent à un dépôt en connexion très altéré; dans ce cas des objets auraient pu être déposés directement sur le corps de l'enfant, quelques pièces glissant par gravitation sous les os longs. Soit les os d'enfant étaient dispersés dans la 
tombe, certains contenus dans un récipient en matière périssable très richement décoré.

Fig. 65 - A. Lot $n^{\circ} 1$ de la structure 7 ; B. Coupes transversales des lots $n^{\circ} 1$ à 3 .
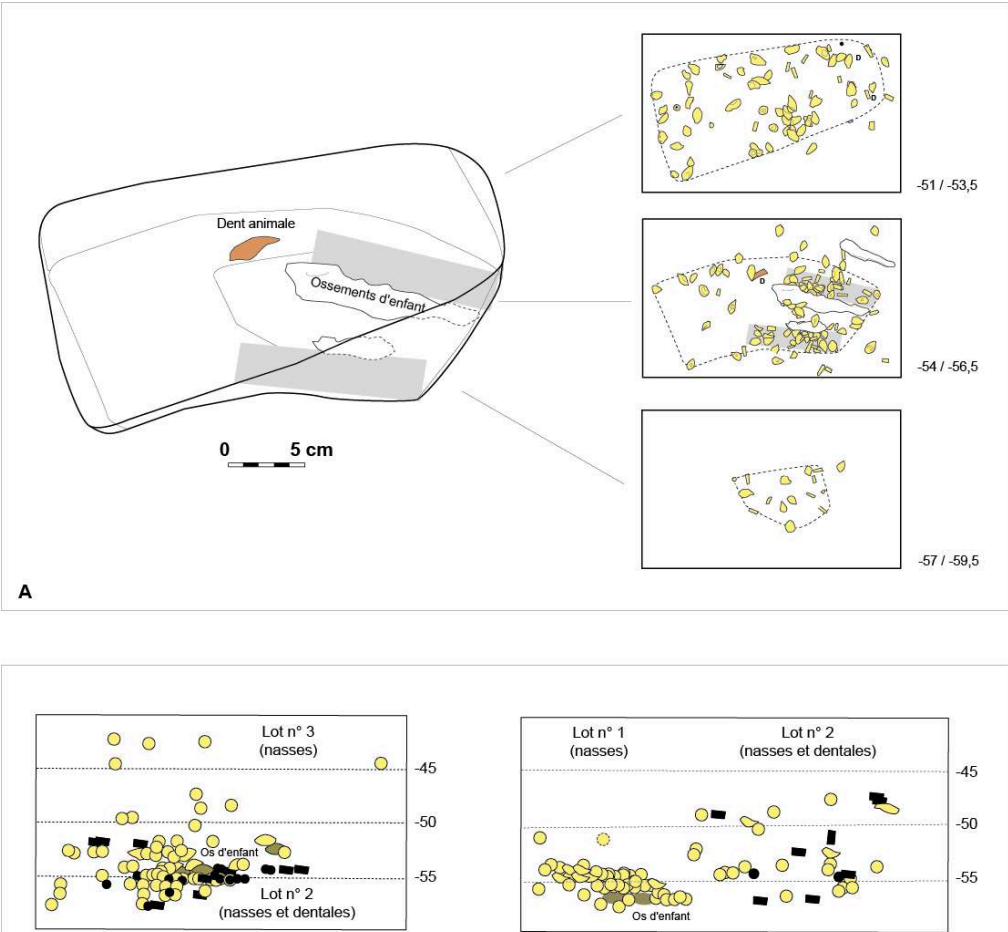

DAO L. Laporte 
Fig. 66 - Lot $n^{\circ} 2$ de la structure 7.
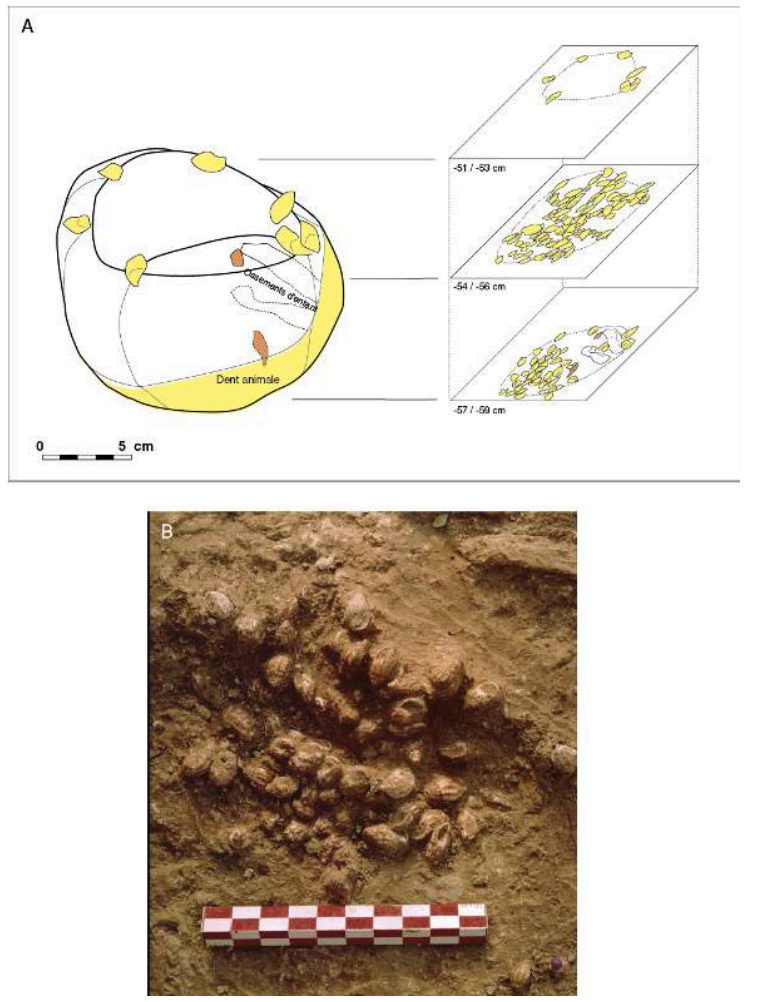

DAO L. Laporte, cliché P. Courtaud.

134 Il nous reste à envisager le cas des pièces situées à l'emplacement des deux individus en connexion. La projection sur un même plan de toutes les pièces concernées ne fait guère ressortir d'information majeure (fig. 67A). Une première approche consiste à aborder l'étude spatiale par niveaux altimétriques. Entre -40 et $-45 \mathrm{~cm}$, soit bien audessus du volume corporel des défunts, l'individu $n^{\circ} 1$ semble couvert par un semis assez lâche de petites coquilles. L'individu $\mathrm{n}^{\circ} 2$ en revanche n'en comporte pratiquement aucune. Une grosse valve est également disposée au-dessus du dos de l'individu $n^{\circ} 1$. Difficiles à interpréter, ces données suggèrent toutefois un traitement différent des deux inhumations, avec peut-être la présence d'un objet en matière périssable couvrant seulement l'un des deux corps? 
Fig. 67 - Coquilles étroitement associées aux volumes corporels des deux individus en connexion dans la structure 7 , démontage par passes altimétriques.

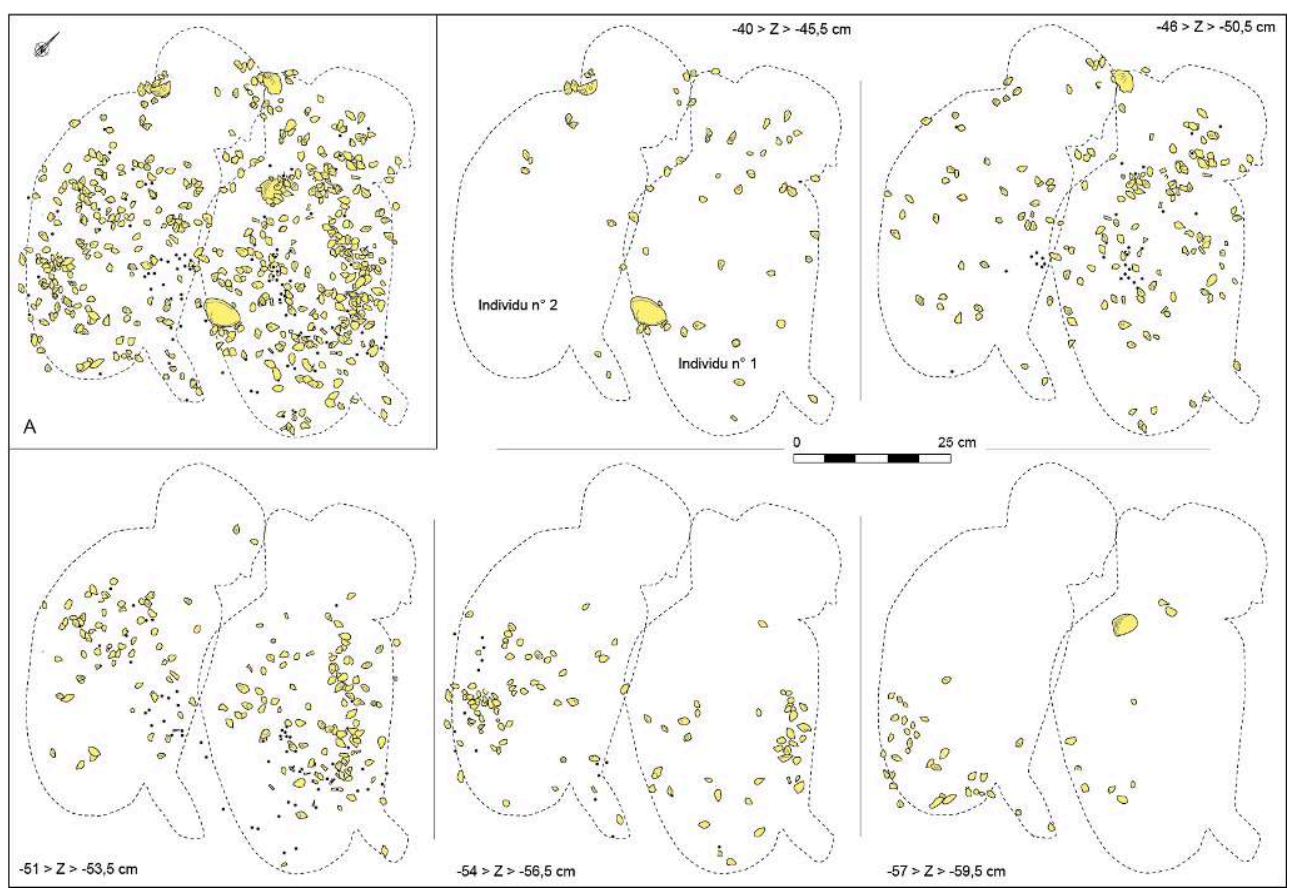

Construction de la figure par L. Laporte, DAO de L. Quesnel pour le dessin de chaque pièce indépendamment, telles que reportées sur les relevés originaux dessinés par $\mathrm{H}$. Duday avec également des annotations de P. Courtaud.

Les objets situés plus bas dans le remplissage sont dans leur très grande majorité associés aux volumes corporels des défunts. Ces derniers ont été déposés sur le côté gauche en position très fortement contractée. Les membres inférieurs fléchis de l'individu $n^{\circ} 2$ s'appuient contre le dos du défunt précédent. Dans les deux cas, leurs épaules s'appuient contre la paroi de la fosse, si bien que les corps ne sont pas disposés horizontalement mais largement inclinés. Les deux crânes sont absents, en partie détruits par le décapage mécanique. La mandibule de chaque individu est toutefois présente. Tous ces éléments influent sur notre façon de traiter l'organisation spatiale de la parure en coquille dans cette tombe. Dans le cas d'objets de parure disposés sur un corps en position fortement repliée, de plus disposé sur un plan incliné, l'organisation initiale de la parure est ainsi particulièrement difficile à reconstituer. Lors de la décomposition des viscères, des masses musculaires et d'éventuelles pièces de vêtement, les objets de parure occuperont les espaces vides ainsi libérés. Même en espace colmaté, certaines pièces pourront notamment avoir tendance à migrer vers le bas avec les sédiments fins environnants. La nature de la matière périssable qui compose l'objet sur lequel elles sont fixées peut également entrer en ligne de compte ; de fines fibres végétales se décomposeront plus vite qu'un cuir épais et tanné. Un terrier, identifié à la fouille près des épaules et du cou de l'individu $n^{\circ} 2$, a de plus considérablement perturbé l'agencement des pièces dans ce secteur.

Pour chaque passe altimétrique, nous avons procédé graphiquement à un démontage simultané des coquilles et des principaux os du squelette. Seule l'excellente qualité, la précision et la fiabilité des relevés comme des informations recueillies sur le terrain ont permis une telle démarche. À titre d'exemple, pour les coquilles situées entre - 46 et $-51 \mathrm{~cm}$ nous avons successivement cartographié celles qui étaient situées au-dessus du 
membre inférieur droit, puis celles situées sur le tronc et sur le membre supérieur droit, lui-même coincé sous le membre inférieur droit (fig. 68). Puis, nous avons fait la même opération pour les objets qui ont glissé dans la cage thoracique ou dans l'abdomen, ceux situés sous le membre supérieur gauche puis sous le membre inférieur gauche. Enfin, nous avons fait la synthèse de ces informations pour toutes les passes altimétriques confondues. Une telle méthode a permis de rendre compte de la place des objets de parure sur les différentes parties du corps tout en maîtrisant la position altimétrique de chaque pièce. Malgré d'inévitables déplacements, l'image obtenue est plus cohérente que ce que l'on aurait pu imaginer au départ.

Fig. 68 - Coquilles étroitement associées aux volumes corporels des deux individus en connexion dans la structure 7 , démontage en fonction des volumes corporels pour une même passe altimétrique.

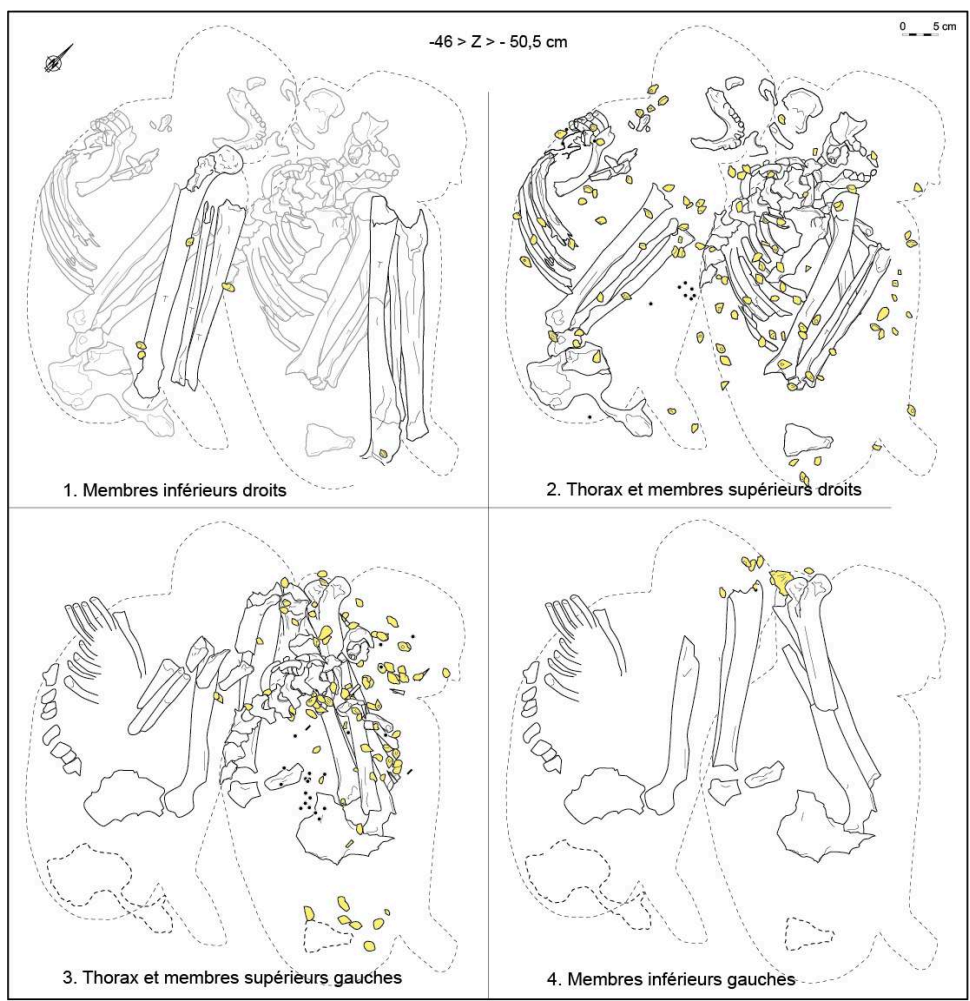

Construction de la figure par L. Laporte, DAO de L. Quesnel pour le dessin de chaque pièce indépendamment, telles que reportées sur les relevés originaux dessinés par H. Duday avec également des annotations de P. Courtaud.

Les coquilles disposées sur le côté droit de chaque individu se concentrent sur une large bande qui couvre le dos et le membre supérieur droit en position repliée sous le membre inférieur gauche (fig. 69). Une telle disposition est particulièrement nette dans le cas de l'individu $n^{\circ} 1$, plus diffuse pour l'individu $n^{\circ} 2$. À titre d'hypothèse, on peut envisager l'existence d'une cape ou d'une bande de tissu maintenant les bras en position repliée contre la poitrine. Une telle "pièce de vêtement » ne couvre pas les membres inférieurs. Certaines coquilles correspondantes semblent avoir glissé entre les côtes, lors de la décomposition des viscères intra-thoraciques et de la mise à plat du thorax, pour s'accumuler à l'intérieur de la cage thoracique (fig. 70A). 
Fig. 69 - Coquilles étroitement associées aux volumes corporels des deux individus en connexion dans la structure 7 , synthèse pour le côté droit.

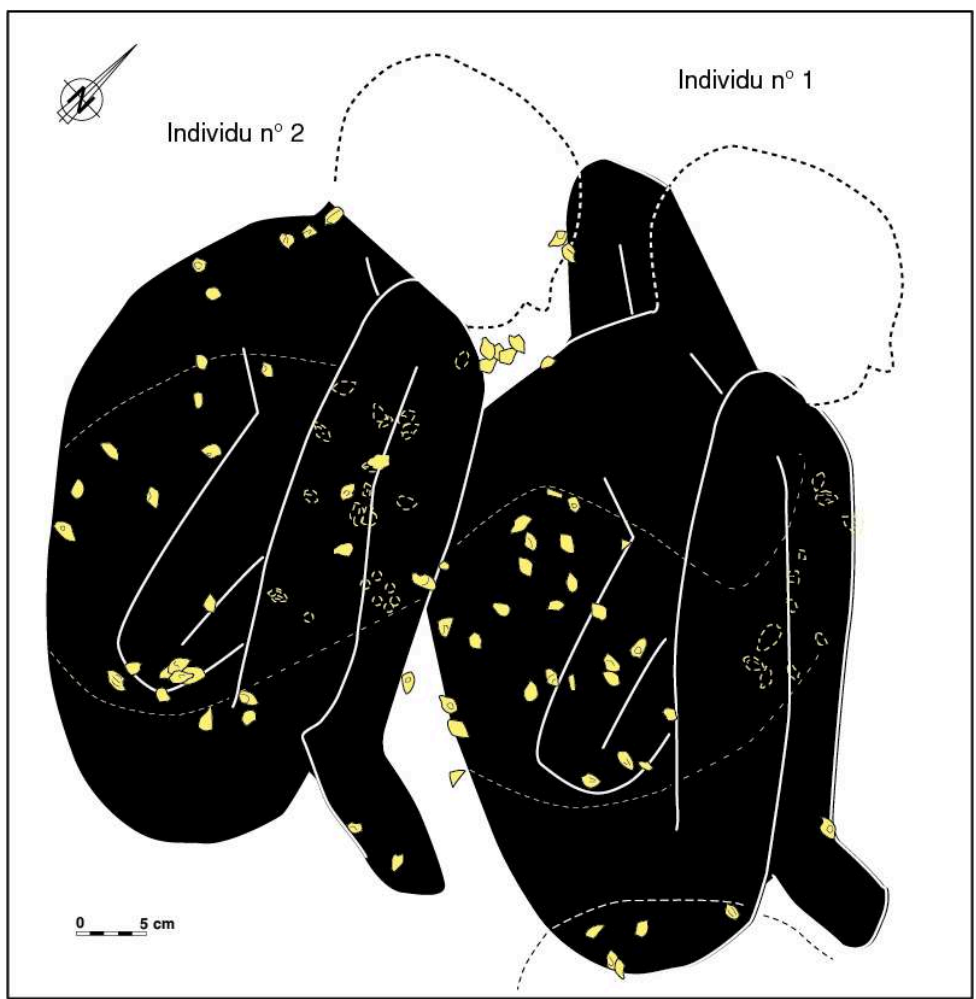

Construction de la figure par L. Laporte, DAO de L. Quesnel pour le dessin de chaque pièce indépendamment, telles que reportées sur les relevés originaux dessinés par $\mathrm{H}$. Duday avec également des annotations de P. Courtaud. 
Fig. 70 - Coquilles étroitement associées aux volumes corporels des deux individus en connexion dans la structure 7 , synthèse pour le côté gauche.
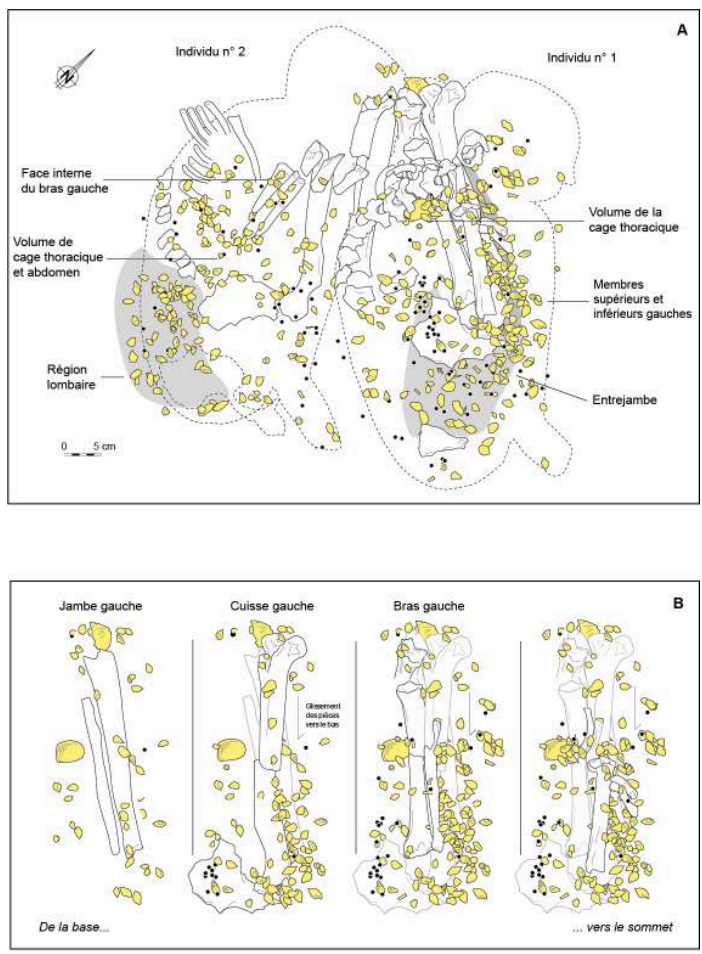

La concentration d'objets de parure part de l'entrejambe et

Construction de la figure par L. Laporte, DAO de L. Quesnel pour le dessin de chaque pièce indépendamment, telles que reportées sur les relevés originaux dessinés par $\mathrm{H}$. Duday avec également des annotations de P. Courtaud.

Un petit lot de coquilles situées sous la scapula droite de l'individu $n^{\circ} 1$ suggère également la présence de parures sur le haut de la poitrine, au moins. Il en va de même pour un petit ensemble d'objets de parure situés sur la face interne du bras gauche replié contre la poitrine de l'individu $n^{\circ} 2$. Un autre lot important de coquilles longe la partie externe du bras gauche de l'individu $n^{\circ} 1$, également en position repliée. Cette accumulation pourrait en partie au moins correspondre à une migration verticale de pièces situées sur les deux avant-bras de ce même défunt. Elle vient ensuite s'intercaler entre le bras gauche et la cuisse gauche, selon une disposition symétrique à celle que nous avons observée sur le côté droit (fig. 70B). L'ensemble serait cohérent avec l'hypothèse d'une bande de tissu, d'une peau ou d'une natte ornée d'un semis de petites coquilles cousues, passant dans le dos du défunt et maintenant ses membres supérieurs contre la poitrine. Cet ensemble a ensuite été recouvert par les membres inférieurs maintenus en position fortement contractée.

De nombreuses coquilles ont également été recueillies dans la région lombaire ou à l'emplacement du bassin des deux individus en connexion. Dans les deux cas, les ailes iliaques sont largement ouvertes et légèrement décalées, l'individu reposant sur la fesse gauche. Il est alors très difficile de restituer la position originelle des pièces dans cet espace. Compte tenu de la position inclinée du corps, une migration vers le bas d'objets de parure situés sur l'abdomen est envisageable. Seul un examen attentif de la position altimétrique des pièces permet ici de se faire une idée très grossière de leur position originelle. Malgré tout, une concentration de coquilles peut être observée dans la région lombaire de l'individu $\mathrm{n}^{\circ} 2$, alors qu'elles semblent plutôt situées en majorité 
sur l'aile iliaque gauche et entre les cuisses de l'individu $n^{\circ} 1$. Cette dernière concentration se trouve d'ailleurs dans la continuité de celle qui longe le bras gauche et repose sur la cuisse gauche de ce dernier. C'est là aussi que se situe la majorité de dentales associée à l'individu $n^{\circ} 1$ (fig. 71). L'hypothèse d'une ceinture dans un cas ou d'un pagne dans l'autre ne serait pas en contradiction avec ces observations. Enfin, il faut citer la présence de deux patelles percées situées respectivement au niveau du genou et du mollet de la jambe gauche de l'individu $n^{\circ} 1$.

Fig. 71 - Dentales étroitement associés aux volumes corporels des deux individus en connexion dans la structure 7 .
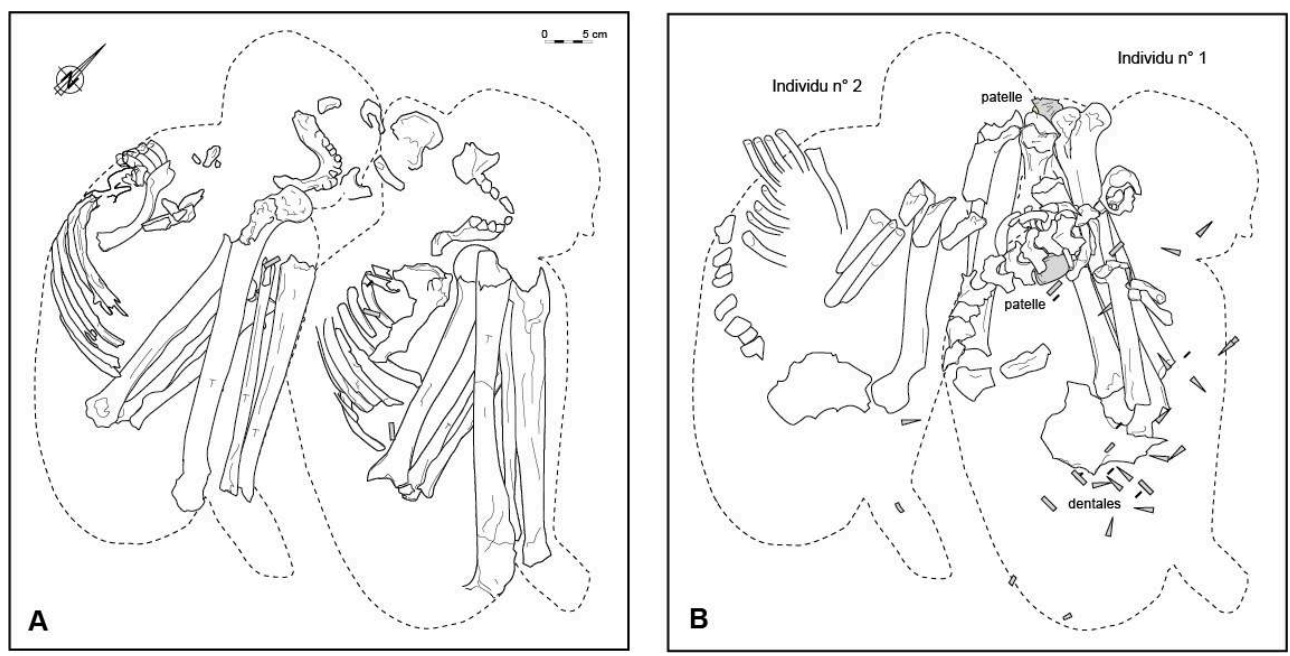

A. Partie droite du corps ; B. Partie gauche du corps.

Construction de la figure par L. Laporte, DAO de L. Quesnel pour le dessin de chaque pièce indépendamment, telles que reportées sur les relevés originaux dessinés par H. Duday avec également des annotations de P. Courtaud.

Les différentes hypothèses formulées précédemment doivent être prises avec prudence compte tenu de la position des corps et de leur forte imbrication. En particulier, ces quelques observations ne suffisent pas à rendre compte de tous les objets de parure recueillis dans le volume corporel des deux défunts. D'autres pièces de vêtement, d'autres parures ou objets en matière périssable ont pu exister que l'on ne peut guère arriver à reconstituer. En définitive seule la présence de coquilles percées sur le haut de la poitrine des deux individus, dans la région lombaire de l'individu $n^{\circ} 2$ ou entre les cuisses de l'individu $n^{\circ} 1$ ainsi que les deux patelles situées le long de la jambe gauche de ce dernier, permet de les attribuer à de la parure corporelle avec quelque assurance. On ignore si l'ensemble était ou non complété par une parure au niveau de la tête. Les coquilles qui initialement devaient couvrir le dos et les avant-bras repliés, en particulier de l'individu $\mathrm{n}^{\circ} 1$, peuvent tout aussi bien avoir été fixées sur une pièce de vêtement proprement dite, susceptible d'être portée par des vivants, que sur un large support en matière périssable destiné, dans le cadre des funérailles, à maintenir les membres supérieurs du défunt en position fortement contractée. Il faut ajouter à cela le nombre important de pièces correspondant à des objets en matière périssable déposés dans la tombe au pied des deux individus précédents. Ainsi, la part de la parure assurément corporelle dans ce dépôt apparait-elle somme toute comme assez réduite. 


\section{La tombe 3}

141 Cette structure n'est que partiellement conservée entre le creusement d'une fosse d'extraction et un fossé protohistorique. Elle a de plus, largement souffert des premiers décapages mécaniques qui en ont arasé les niveaux supérieurs. Au moment de la découverte, certains éléments ont été démontés avant l'intervention des anthropologues sur le terrain. Quelques photos permettent toutefois de se faire une idée de l'organisation des vestiges à ce niveau. Dans un premier temps, une femme adulte et grand enfant ont été déposés simultanément en position assise dans une petite fosse. Seul le membre inférieur gauche et une partie du bassin du grand enfant, ainsi qu'une partie du membre inférieur droit et le bassin de la femme adulte ont pu faire l'objet d'une fouille détaillée. Le troisième individu a été inhumé postérieurement dans une fosse qui recoupe la précédente. Comme cela a immédiatement été remarqué au cours de la fouille, les objets de parure sont exclusivement associés aux deux premiers individus. Compte tenu de son état de conservation, la richesse en objets de parure de cette tombe n'en est que plus frappante avec 865 objets en coquille recueillis. Presque toutes les pièces ont été dessinées à la main sur douze relevés successifs, à l'exception de celles recueillies juste après le passage de la pelle mécanique.

La répartition des objets de parure en coquille dans le remplissage de la structure 3 semble à première vue aléatoire, si ce n'est une plus grande densité de pièces aux endroits où la fosse est un peu plus profonde (fig. 72). On remarquera cependant que les coquilles de grande taille sont toutes dans la moitié sud du remplissage. L'arasement de cette structure, comme l'enchevêtrement des corps sur un espace de superficie restreinte, ne facilite pas l'interprétation des données spatiales. Les volumes corporels des deux individus, ou du moins ce qu'il en reste, occupent tout l'espace disponible, sans que l'on puisse isoler, comme dans le cas précédent, des ensembles de parures qui ne soient pas directement associés aux défunts. La superposition des douze relevés effectués illustre clairement cet état de fait (fig. 73A). 
Fig. 72 - Répartition des coquilles dans la structure 3.

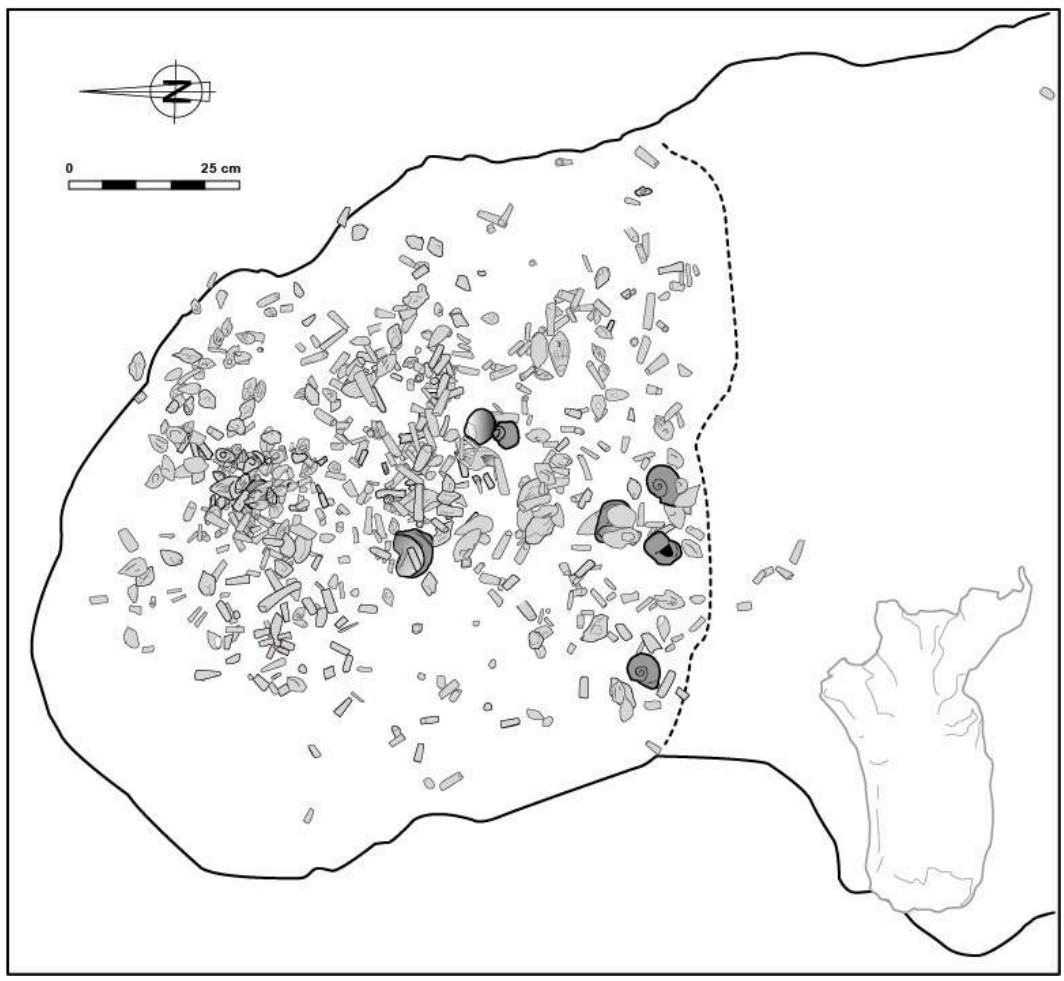

Construction de la figure par L. Laporte, DAO de L. Quesnel pour le dessin de chaque pièce indépendamment, telles que reportées sur les relevés originaux dessinés par $\mathrm{H}$. Duday avec également des annotations de P. Courtaud. 
Fig. 73 - Imbrication des ossements humains et des coquilles dans la structure 3.
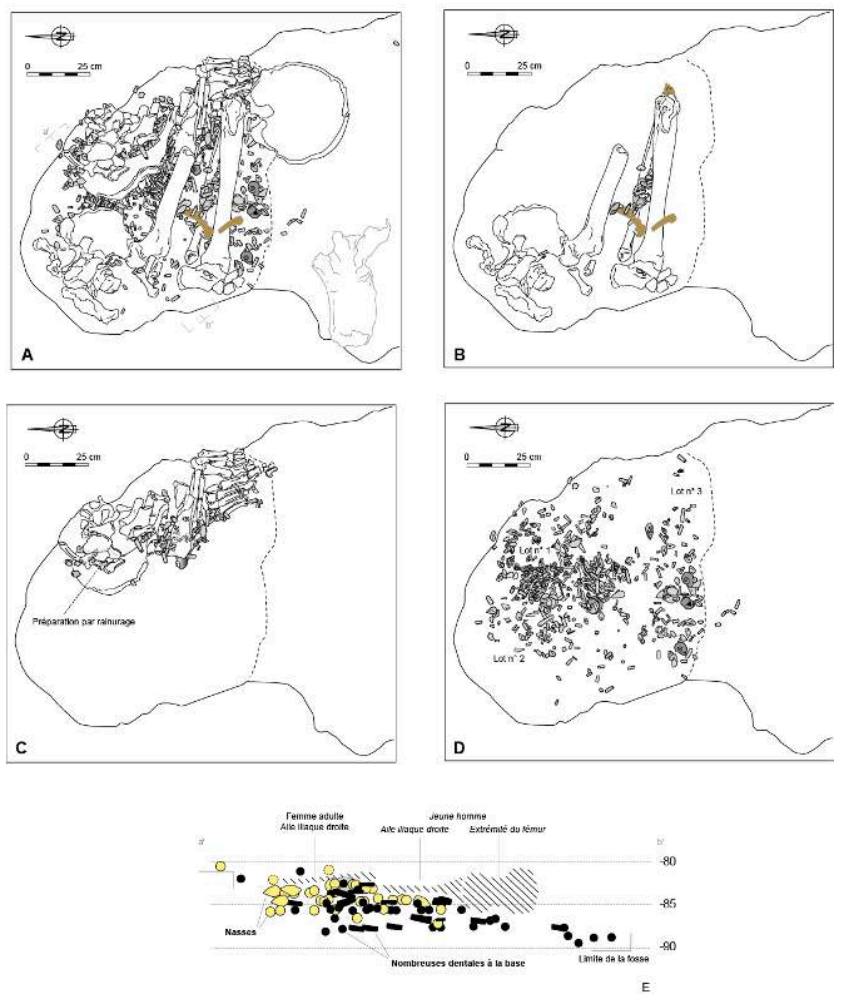

A. Projection horizontale de toutes les pièces ; B. Sélection des pièces disposées sur la jambe du grand enfant ; c. Sélection des pièces disposées sur les os de la femme adulte; D. Sélection des pièces situées sur le fond de la fosse et sous les os des deux individus précédents ; E. Coupe transversale a' b'.

Construction de la figure par L. Laporte, DAO de L. Quesnel pour le dessin de chaque pièce indépendamment, telles que reportées sur les relevés originaux dessinés par $\mathrm{H}$. Duday avec également des annotations de P. Courtaud.

Nous avons alors procédé à des démontages partiels, en ne gardant par exemple que les objets de parure disposés sur la jambe gauche du grand enfant (fig. 73B). Puis, le même procédé a été appliqué aux coquilles situées sous le membre inférieur droit et sur le bassin ou les pieds de la femme adulte (fig. 73C). Dans le premier cas on remarquera la présence de nombreuses nasses de grande taille, alors que dans le second les amas de dentales sont particulièrement nombreux. Quelques rares pièces se trouvaient étroitement imbriquées avec les vertèbres de la femme adulte. Dans ce cas, il s'agit assurément de parure corporelle. Parmi ces dernières pièces, il en est qui se distinguent par un mode de façonnage très particulier, avec une préparation de la perforation par rainurage.

Les quelques coquilles qui se trouvaient dans les espaces interstitiels et toutes celles, nombreuses, placées sous les ossements des deux individus ont ensuite été cartographiées à part (fig. 73D). Trois concentrations apparaissent alors. Un premier lot semble disposé en couronne sur le fond de la fosse, contre sa paroi orientale. Il jouxte un second lot de forme grossièrement rectangulaire situé au centre de cette dernière. Un dernier lot, orienté est-ouest, vient s'appuyer contre et passe sous l'emplacement de la jambe gauche du grand enfant. Nous avons ensuite procédé à une cartographie des pièces situées sous les deux corps, par passes altimétriques (fig. 73E). À chaque fois, il 
nous est apparu intéressant de distinguer la répartition des dentales de celles des nasses et des autres coquilles, souvent de taille nettement plus importante.

$\mathrm{Au}$ niveau $-81,5 /-83,5 \mathrm{~cm}$, la plupart des pièces sont disposées sous le bassin de la femme adulte (fig. 74). Quelques dentales semblent former un léger effet paroi que l'on retrouvera de façon beaucoup plus marquée dans le décapage suivant. Entre -83,5 et $-87,5 \mathrm{~cm}$ de profondeur, un lot de dentales apparaît très nettement au centre de la fosse, où il semble limité par deux effets de parois parallèles. Ce lot est situé sous le bassin des deux individus inhumés. Au sud, quelques dentales semblent orientés parallèlement à l'axe de la jambe gauche du jeune homme grand enfant. Les dentales qui reposent directement sur le fond de la fosse forment un tapis assez lâche et sans organisation évidente. En ce qui concerne les nasses, on remarque une concentration sous le bassin de la femme adulte entre $-81,5$ et $-83,5 \mathrm{~cm}$ de profondeur. Ce lot semble se prolonger en arc de cercle autour de l'aile iliaque. Au décapage suivant, entre -83,5 et $-87,5 \mathrm{~cm}$, on observe à cet emplacement une disposition en couronne de nasses souvent regroupées par paires. À la cote -84, les nasses forment deux files perpendiculaires, autour d'une coquille de nérite (fig. 75). Un dentale est situé dans l'axe de la perforation de cette dernière, comme si un seul lien reliait l'ensemble. À la cote -85, des nasses de petite taille regroupées par paires dessinent très nettement un arc de cercle déjà détecté dans les décapages précédents. À l'ouest de ces dernières, un petit groupe de dentales commence à apparaître. Ils annoncent les nombreux dentales situés au niveau -86 et contribuent à l'effet de paroi que nous avons déjà décrit. Deux structures semblent se superposer localement. La première, de forme circulaire et d'une vingtaine de centimètres de diamètre, est principalement marquée par la présence de nasses de petite taille regroupées par paires. La seconde constitue une bande d'une trentaine de centimètres de large, principalement marquée par la présence de nombreux dentales. Elle semble buter contre la cuisse gauche du grand enfant. À l'emplacement de cette jambe gauche, on remarquera la présence de coquilles de nasses de taille nettement supérieure aux précédentes et de plusieurs coquilles de grand format (natices). 
Fig. 74 - Répartition spatiale des nasses et des dentales dans la structure 3, par passes altimétriques.
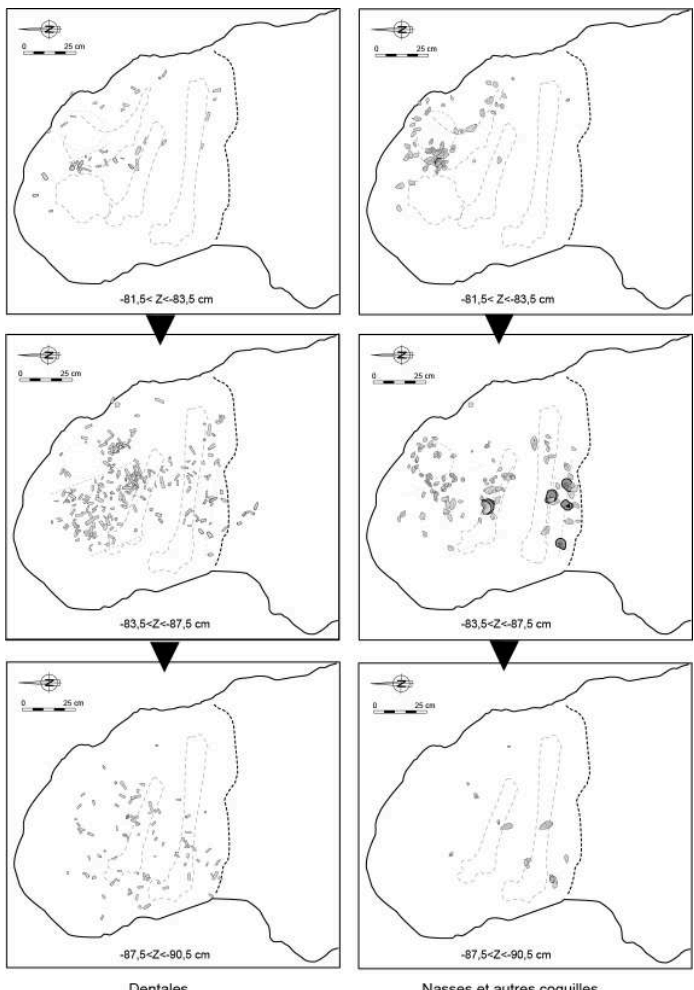

Construction de la figure par L. Laporte, DAO de L. Quesnel pour le dessin de chaque pièce indépendamment, telles que reportées sur les relevés originaux dessinés par $\mathrm{H}$. Duday avec également des annotations de P. Courtaud.

Fig. 75 - Composition du lot $n^{\circ} 1$ de la structure 3 par passes altimétriques.
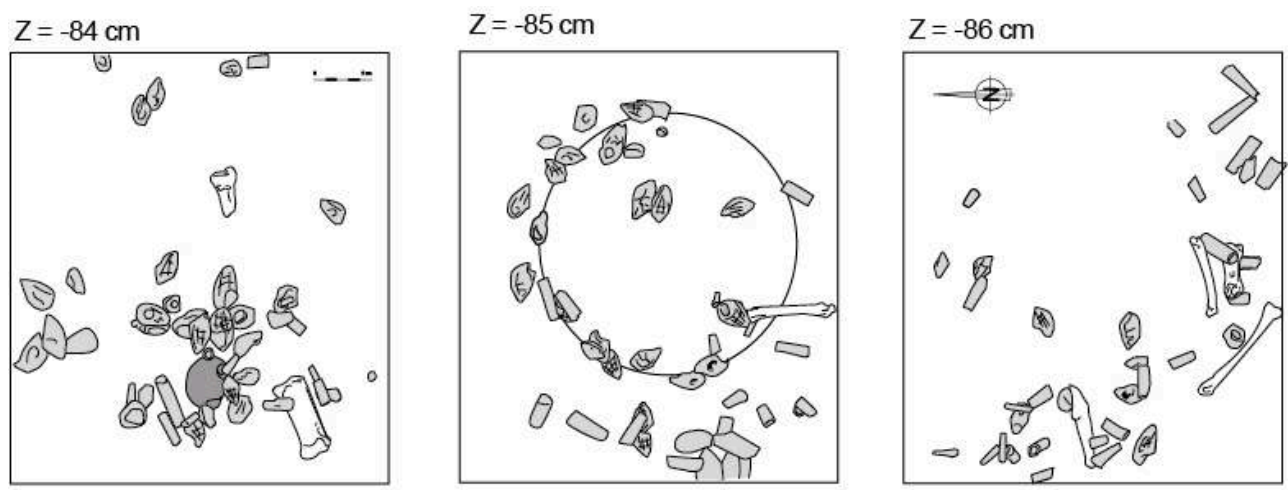

Construction de la figure par L. Laporte, DAO de L. Quesnel pour le dessin de chaque pièce indépendamment, telles que reportées sur les relevés originaux dessinés par $\mathrm{H}$. Duday avec également des annotations de P. Courtaud.

De telles structurations spatiales paraissent encore plus évidentes lorsque l'on rassemble les différentes informations détaillées ci-dessus. En ce qui concerne les dentales, on remarquera que l'un des deux effets de paroi tend à se prolonger vers le sud par une ligne de dentales qui recouvre notamment le pied droit de la femme adulte (fig. 76A). À titre d'hypothèse, on peut envisager que les deux individus aient été déposés assis sur une même natte, ou tout autre objet en matière périssable notamment décoré de nombreux dentales. Celle-ci viendrait s'appuyer sur les pieds de la femme 
adulte et contre la jambe gauche du grand enfant. Par ailleurs, une photo prise lors des tout premiers décapages, montre trois rangées de dentales superposées (fig. 76B). Elles sont disposées en épi et, chaque rang présente une orientation perpendiculaire à celle du rang supérieur.

Fig. 76 - Dentales situés à la base du remplissage de la structure 3, éléments de synthèse.
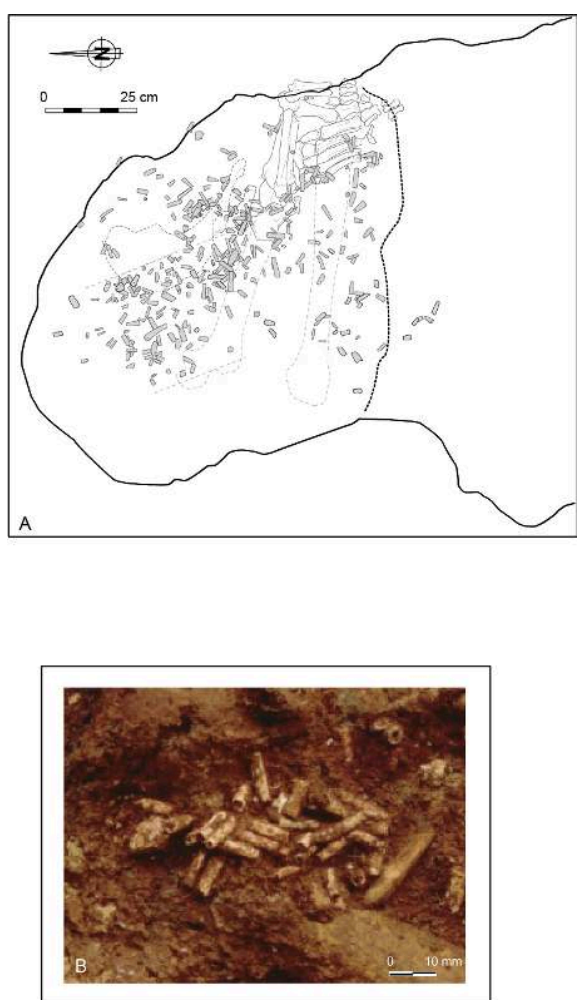

A. Construction de la figure par L. Laporte, DAO de L. Quesnel pour le dessin de chaque pièce indépendamment, telles que reportées sur les relevés originaux dessinés par $\mathrm{H}$. Duday avec également des annotations de P. Courtaud ; B. Cliché P. Courtaud.

En ce qui concerne les nasses, elles se répartissent clairement en deux ensembles (fig. 77A). L'un constitué de pièces de petite taille qui semble correspondre à la présence d'un objet de forme circulaire placé sous les fesses de la femme adulte. L'autre constitué de pièces d'une taille nettement supérieure, étroitement liées au membre inférieur gauche du grand enfant. Dans la structure 3, sur 23 coquilles de nasses dont la taille appartient au quartile supérieur, toutes structures confondues, 18 sont concentrées autour de cette jambe. Trois coquilles de grands gastéropodes sont de plus alignées le long du mollet. 
Fig. 77 - Nasses et gastéropodes dans le remplissage de la structure 3 , éléments de synthèse. Pour l'échantillon correspondant à l'histogramme, NR = 70 .
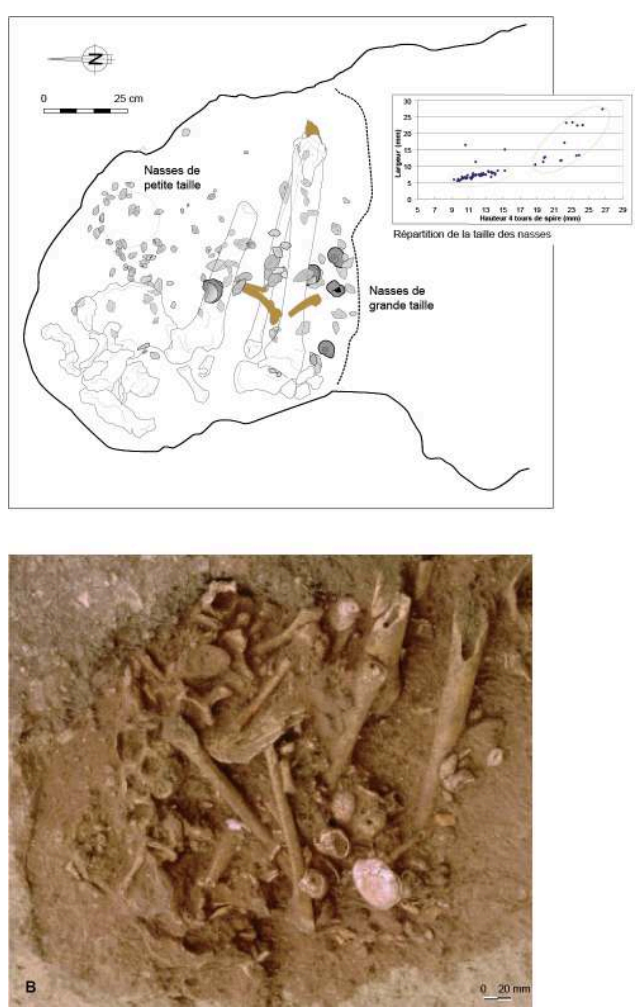

A. DAO L. Laporte et C. Dupont pour la figure, L. Quesnel pour le dessin de chaque pièce indépendamment ; B. Cliché P. Courtaud.

On remarquera que ces différentes pièces enveloppent totalement la jambe, avec une concentration importante située au-dessus de la cheville. Cette concentration s'achève par la présence de quelques os d'un petit mammifère. Ignorant sa détermination spécifique on ne sait s'il s'agit d'un animal à fourrure. Une canine percée se trouve au niveau du genou. On retrouve ensuite une concentration analogue au niveau de la cuisse. Si seules les pièces situées sous le fémur ont été dessinées, les photos prises lors des premiers décapages permettent d'affirmer que ces éléments de parure entouraient totalement le haut de la cuisse (fig. 77B). Quelques coquilles de grands gastéropodes ou de bivalves étaient également présentes à ce niveau. L'hypothèse d'objets de parure disposés sur une pièce de vêtement, type jambière, peut être ici raisonnablement proposée. On ne peut malheureusement pas valider ces résultats par une confrontation avec la parure associée à la jambe droite qui a disparu.

Si nous avons provisoirement dissocié l'analyse spatiale des dentales, de celles des coquilles percées, elles sont cependant toutes représentées dans les ensembles que venons de décrire, bien qu'en proportions très différentes. L'analyse spatiale des traces d'usure sera l'occasion d'illustrer ces différentes associations. Les pièces dégagées à proximité de la jambe gauche du grand enfant en offrent les meilleurs exemples ; toutes les traces d'usure observées à la loupe binoculaire ont été reportées sur le plan de la figure 78. On remarque ainsi au moins deux cas où l'usure du bord de la perforation d'une nasse se trouve dans le prolongement de l'ouverture d'un dentale adjacent. Elles pourraient avoir été fixées par un même lien. D'autres nasses semblent associées deux par deux, fixées soit par un seul et même lien, soit séparément les unes des autres. 
Certains segments de dentales sont emboîtés, sans doute fixés par un seul et même lien. Enfin, près des trois quarts des dentales présentant des traces d'abrasion latérales $(11 / 16)$ sont également regroupées à cet endroit. Parmi la trentaine de nasses portant une usure particulièrement accentuée, quelques-unes sont également situées autour du mollet gauche du grand enfant. Mais la plupart appartiennent au lot $n^{\circ} 1$, situé principalement sous le bassin de la femme adulte. Certaines des pièces de vêtement ou des objets décorés déposés dans la tombe semblent donc avoir été longuement portés, ou utilisés, au préalable.

Fig. 78 - Éléments de parure situés au niveau de la jambe gauche du grand enfant déposé dans la structure 3 .

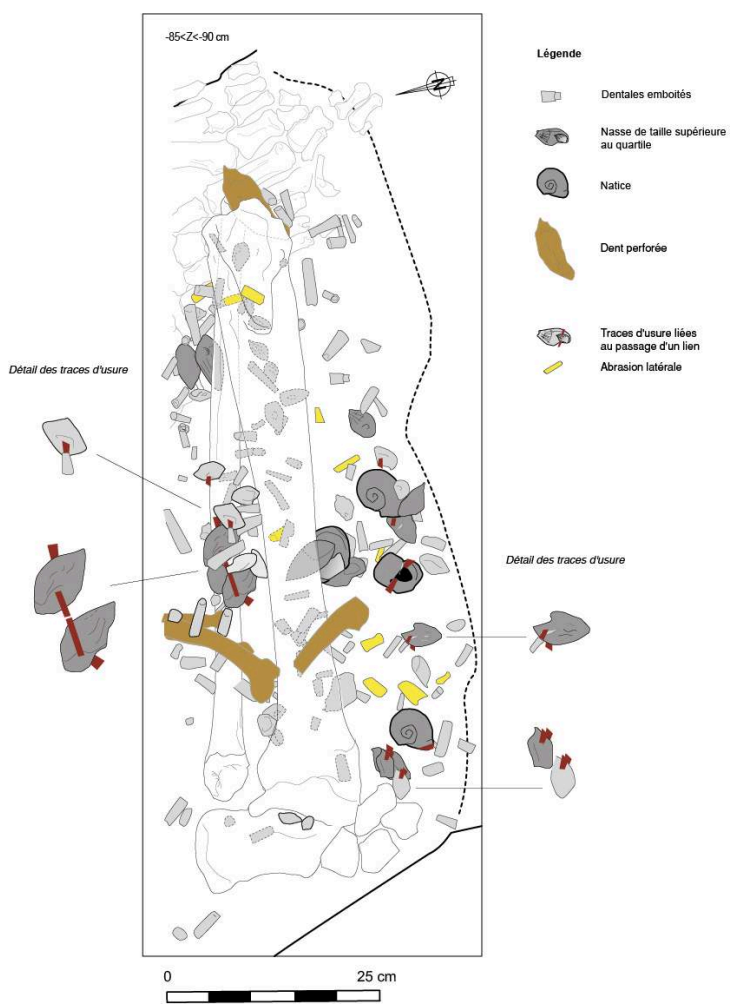

Construction de la figure par L. Laporte, DAO L. Quesnel pour le dessin de chaque pièce indépendamment, telles que reportées sur les relevés originaux dessinés par H. Duday avec également des annotations de P. Courtaud.

$\mathrm{Au}$ sein de cette tombe, un nombre important de pièces semble correspondre à la présence d'objets en matière périssable richement ornés. La surreprésentation des dentales dans la structure 3 pourrait notamment s'expliquer ainsi. D'autres pièces percées sont plus étroitement liées aux volumes corporels. Certaines pourraient avoir été fixées sur des pièces de vêtement, notamment autour des jambes du grand enfant. Dans ce cas précis, les objets de parure corporelle se distinguent soit par la nature des coquilles représentées (natices, nérites), soit par une sélection de la taille des coquilles de nasses utilisées, voire pour certaines de ces dernières par un mode de façonnage original des perforations (préparation par rainurage). 


\section{La tombe 10}

151 Cette tombe est sans doute la mieux conservée de toutes celles qui ont été mises au jour dans la nécropole. Elle ne contenait les restes que de deux individus, ceux d'un adulte et d'un enfant, ce qui facilitera l'analyse des coquilles percées associées. Contrairement aux cas précédents, l'intégralité du corps de l'adulte en connexion a pu être dégagée. C'est là aussi un atout pour l'étude de la parure correspondante. 435 pièces en coquille ont été recueillies dans cette fosse dont le remplissage a été fouillé sur une vingtaine de centimètres d'épaisseur. La grande majorité a été dessinée sur douze relevés successifs. Seules quelques dizaines de pièces ont été situées sur une photographie verticale.

Dans les parties supérieures du remplissage, entre les cotes -35 et $-39,5 \mathrm{~cm}$, quelques pièces seulement apparaissent sous la forme d'un semis apparemment aléatoire (fig. 79). Juste en dessous, entre -40 et $-44,5 \mathrm{~cm}$, une première concentration d'éléments de parure est étroitement associée aux ossements d'un enfant déposé contre le dos de l'adulte, qui repose sur le côté gauche en position contractée. Un bucrane d'aurochs est déposé au-dessus des pieds de ce dernier, également associé à quelques pièces percées éparses. Un semis lâche d'éléments de parure commence à apparaître en avant de la face de l'individu adulte. C'est à cet emplacement que l'on trouvera une nouvelle concentration d'objets percés au décapage suivant, entre -45 et $-49,5 \mathrm{~cm}$. Cette dernière comprend quelques dentales, des nasses, un lot de coquilles de taille nettement plus importante et quelques dents percées qui font l'objet d'une étude séparée. Quelques valves sont également disposées autour du crâne, alors qu'apparaissent les premières pièces percées directement associées au volume corporel de l'adulte inhumé dans cette tombe. Quelques coquilles seront toujours présentes au sein même du volume corporel, à la base du remplissage soit entre -50 et $-54,5 \mathrm{~cm}$. On remarquera surtout à ce niveau, une concentration de coquilles percées située sous le crâne, dans le prolongement de celles déjà repérées en avant de la face. À partir de cette première approche nous étudierons d'abord la répartition spatiale des pièces percées étroitement associées aux os de l'enfant, puis celles situées autour du bucrane ou devant la face de l'adulte, pour aborder enfin celles qui s'inscrivent au sein du volume corporel de ce dernier. 
Fig. 79 - Répartition des coquilles dans la structure 10, démontage par passes altimétriques.
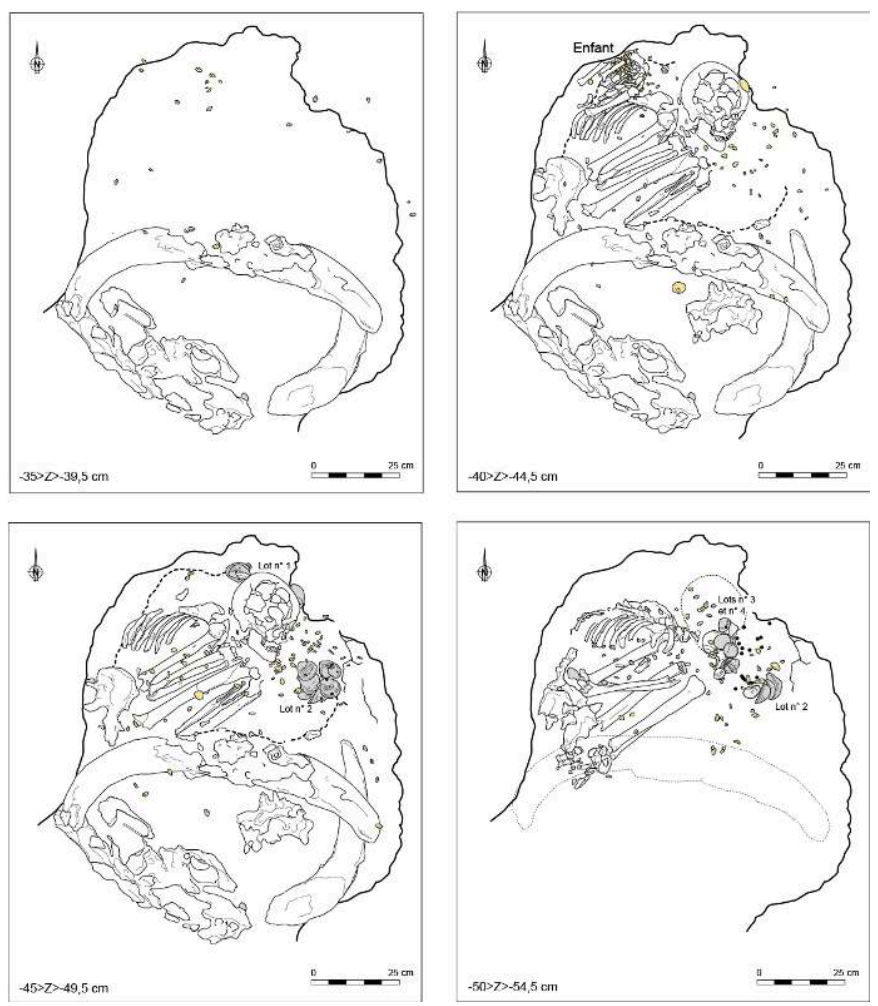

Construction de la figure par L. Laporte, DAO L. Quesnel pour le dessin de chaque pièce indépendamment, telles que reportées sur les relevés originaux dessinés par H. Duday avec également des annotations de P. Courtaud.

Les ossements de l'enfant et les coquilles associées s'inscrivent dans un volume de section globalement rectangulaire, où ils sont parfois enchevêtrés (fig. 80B). Si l'on ne garde plus sur le même plan que les pièces percées, on s'aperçoit que certaines sont alignées sur les côtés les plus longs de ce volume, comme sur les parois d'un contenant (fig. 80A). Un tel effet n'est véritablement visible qu'en superposant des pièces réparties sur $7 \mathrm{~cm}$ d'épaisseur, comme si de chaque côté elles étaient placées sur un même plan vertical. Cette disposition suppose que les pièces étaient fixées sur un support en matière périssable relativement fin, ouvert ou perméable, en espace colmaté. Dans le cas contraire, toutes les pièces auraient glissé verticalement ou se seraient effondrées à l'intérieur des espaces vides ménagés par le contenant. Remarquons que les dentales constituent l'essentiel des coquilles disposées sur le flanc sud, alors que ces dernières sont absentes du flanc nord du volume concerné (fig. 80C). Au centre en revanche, un groupe de nasses est étroitement mêlé aux ossements humains. C'est ce que fait clairement apparaître le démontage par relevés de fouille successifs, indépendamment de l'altitude des pièces (fig. 80D). On ne sait trop s'il faut envisager cette dernière concentration en termes d'ornementation corporelle, mais cela ne peut être exclu. Le corps de l'enfant, peut-être associé à des éléments de parure corporelle, semble donc avoir été disposé dans un contenant en matière périssable de section plutôt rectangulaire, lui-même orné de nombreuses coquilles. Une telle disposition, particulièrement nette dans le cas présent, nous renvoie aux observations similaires effectuées pour le lot $n^{\circ} 2$ de la structure 10 . 
Fig. 80 - Coquilles étroitement associées aux restes osseux de l'enfant déposé dans la structure 10 .
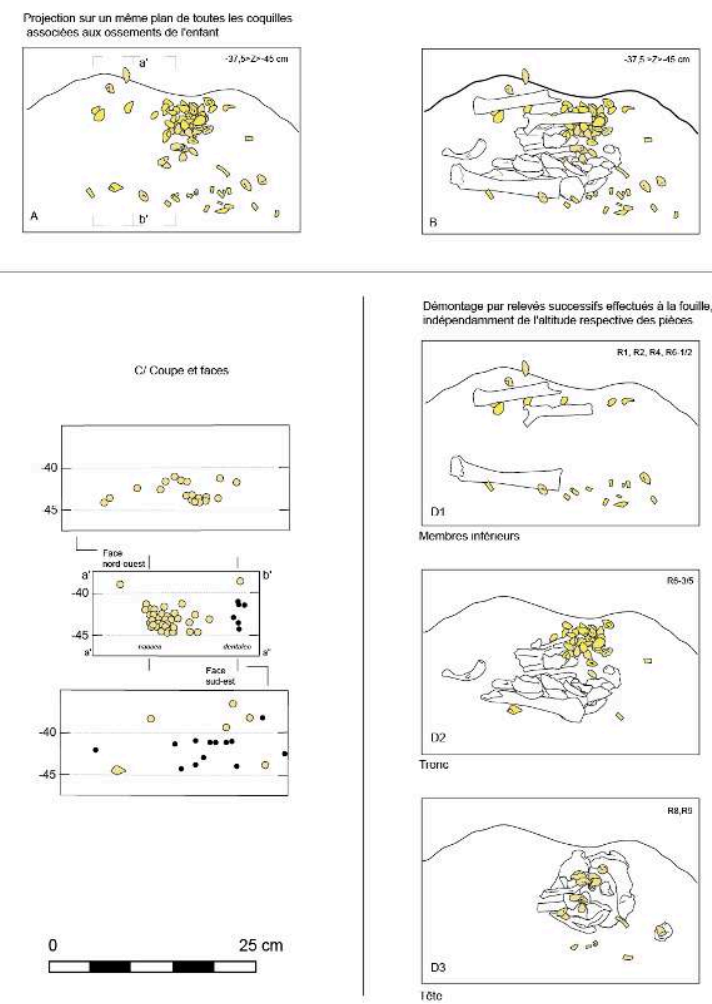

Construction de la figure par L. Laporte, DAO de L. Quesnel pour le dessin de chaque pièce indépendamment, telles que reportées sur les relevés originaux dessinés par H. Duday avec également des annotations de P. Courtaud.

Nous avons également été surpris de constater la présence de quelques coquilles juste au-dessus et tout autour de la partie centrale du bucrane déposé sur les pieds de l'adulte (fig. 81). Ce semis assez lâche de pièces percées, présentes à différentes profondeurs, enveloppe en fait la partie supérieure du bucrane, un peu comme si elles avaient été fixées sur un support en matière périssable recouvrant sur ce dernier. On remarquera que l'unique patelle concernée est alors placée en position axiale. Un petit groupe de coquilles suit par ailleurs la courbure du massacre d'aurochs sous-jacent, au niveau de l'une des extrémités de ce dernier. La pointe de cette corne avait-elle été enveloppée d'une ornementation particulière? L'ensemble aurait-il pu faire partie d'une parure destinée pour l'occasion à la bête immolée ? Il est certain en tout cas qu'un tel dispositif aurait participé à la mise en scène de ces pièces spectaculaires que constituent les deux bucranes superposés, lors de l'inhumation du défunt. 
Fig. 81 - Coquilles présentes au niveau des bucranes déposés dans la structure 10.

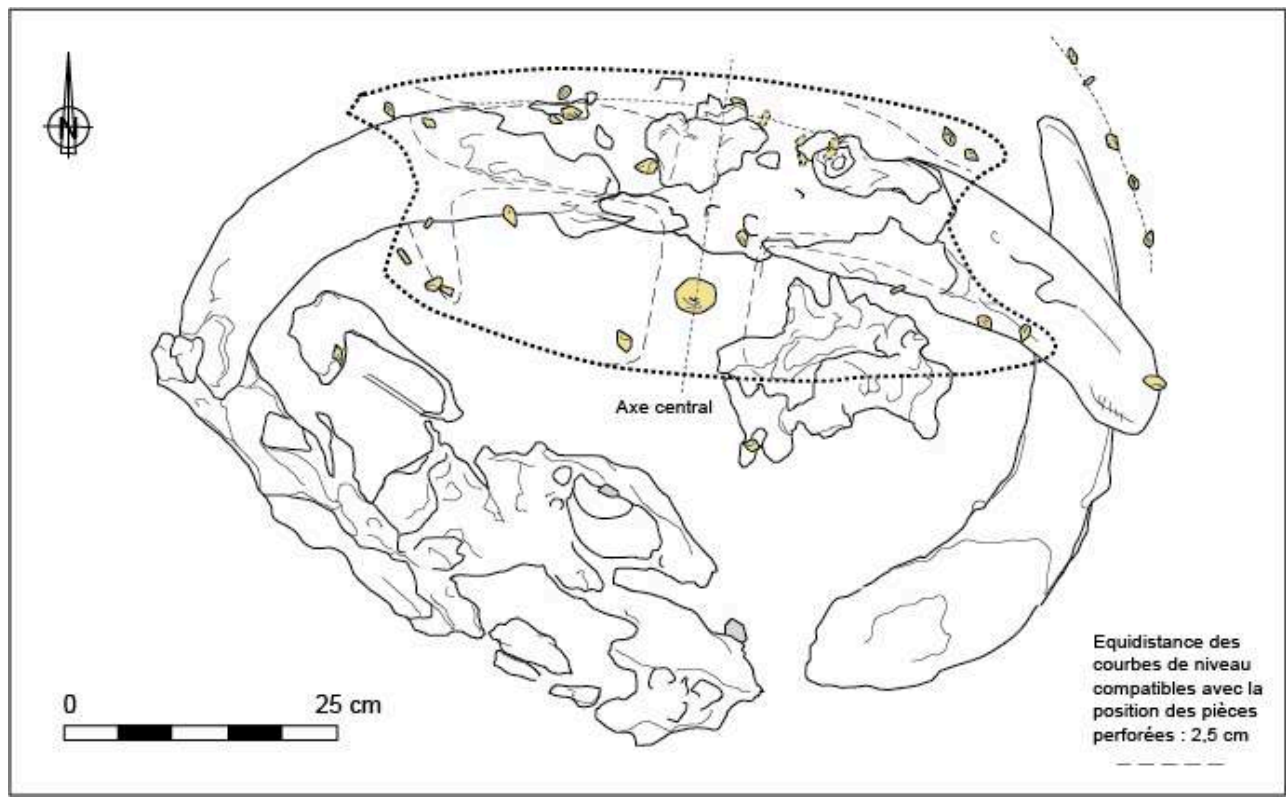

Construction de la figure par L. Laporte, DAO de L. Quesnel pour le dessin de chaque pièce indépendamment, telles que reportées sur les relevés originaux dessinés par H. Duday avec également des annotations de P. Courtaud.

Abordons maintenant les coquilles situées près du crâne et un peu en avant de la face de l'adulte. Si l'on reporte sur un même plan horizontal toutes les pièces percées contenues dans l'épaisseur du remplissage pour ce secteur particulier, on observe un effet de paroi très nettement marqué (fig. 82A). Il isole un lot important de pièces situées dans le coin nord-est de la tombe. On y observe deux ensembles de coquilles de taille nettement plus importante. Le premier ensemble est associé presque exclusivement à des nasses, souvent de taille largement supérieure à la moyenne, alors que le second présente en surface un nombre élevé de dentales. Ces deux concentrations sont séparées par un espace de faible densité. Une telle structuration spatiale se surimpose cependant aux genoux, aux épaules et à la tête de l'adulte. Il convient donc d'opérer un démontage plus détaillé avant toute interprétation hâtive de ces différentes observations. 
Fig. 82 - Coquilles présentes autour du crâne et en avant de la face de l'individu adulte déposé dans la structure 10 .

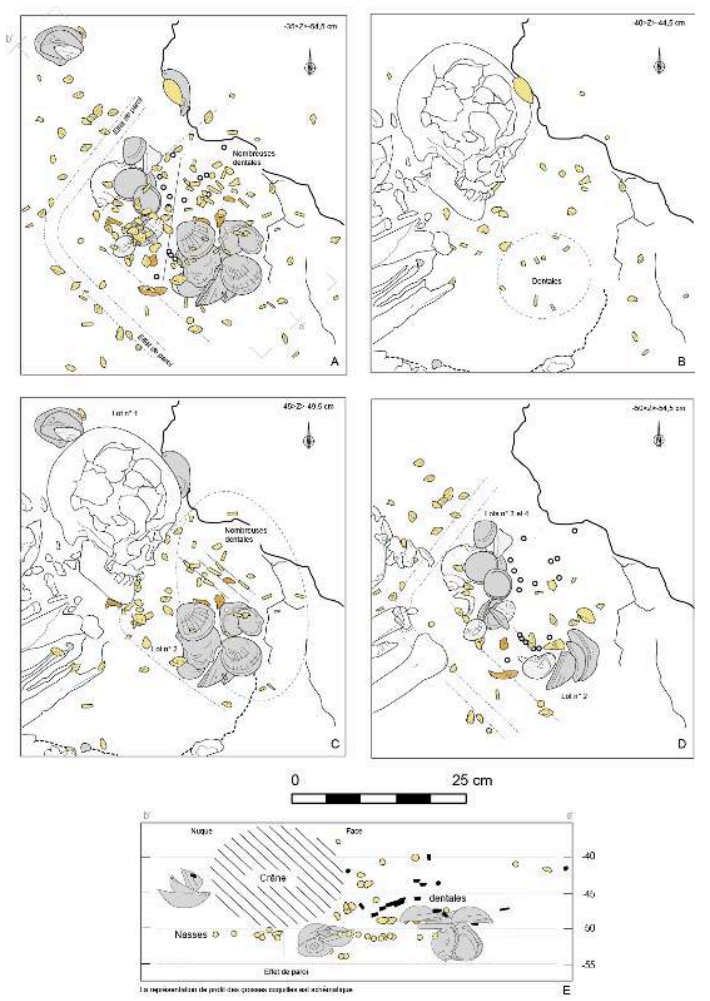

Construction de la figure par L. Laporte ; DAO de L. Quesnel pour le dessin de chaque pièce indépendamment, telles que reportées sur les relevés originaux dessinés par H. Duday avec également des annotations de P. Courtaud.

Entre -40 et $-44,5 \mathrm{~cm}$ seules quelques coquilles, dont une petite concentration de dentales, apparaissent (fig. 82B). Une nasse de taille largement supérieure à la moyenne est plaquée contre le crâne, sur le frontal. Une valve de Laevicardium crassum se trouve juste en dessous, également plaquée contre le frontal, dont sa face interne épouse la courbure. Au même niveau, entre -45 et $-49,5 \mathrm{~cm}$, un autre ensemble de valves est situé près de l'occipital (lot $\mathrm{n}^{\circ} 1$ ). Il s'agit de deux valves de Laevicardium crassum encastrées, surmontées par une coquille de patelle. Les deux valves sont opposées par leur charnière, avec la perforation dirigée vers le haut (fig. 3.59). Elles pouvaient être suspendues par un lien. La patelle, percée au sommet, présente une déformation par usure du bord de la coquille. Cela suggère que la coquille a pu frotter contre un lien ou un support quelconque à cet emplacement. Une nasse isolée, venue se glisser contre la valve inférieure, complète l'ensemble.

Un peu en avant de la face, un deuxième ensemble de valves (lot $n^{\circ} 2$ ) est entouré de nombreux dentales (fig. $82 \mathrm{C}$ ). Plus de la moitié des dentales recueillis dans la structure 10 sont regroupés dans ce secteur. Parmi ces derniers, certains sont disposés sur trois lignes parallèles équidistantes d' $1 \mathrm{~cm}$ environ. Quelques-uns sont emboîtés ce qui suggère qu'ils aient pu être enfilés sur un même lien. D'autres, au sein du même alignement, portent les traces d'une forte abrasion latérale qui atteste leur fixation sur un support en matière périssable. Ces deux observations ne sont pas contradictoires. Elles témoignent de la présence d'un objet en matière périssable sur lequel étaient cousues au moins trois lignes parallèles de dentales alignés. 
$158 \mathrm{Au}$ décapage suivant, entre -50 et $-54,5$, le crâne a été retiré. À la base du lot $n^{\circ} 2$ on observe d'autres valves et quelques files de nasses percées qui semblent parfois se situer dans le prolongement de leur perforation. Un dernier ensemble de valves (lots $n^{\circ} 3$ et 4) se situe exactement sous l'emplacement du crâne. L'une des nasses qui leur est associée est d'ailleurs recouverte par un fragment de vertèbre cervicale. C'est à ce niveau-là, pour les pièces situées sur le fond de la fosse et sous les os du squelette, que l'on observe le plus nettement l'effet de paroi déjà cité (fig. 82 E). Ce dernier n'est donc pas un artifice, mais bien la marque d'une paroi ou d'un objet en matière périssable qui isole l'ensemble des pièces percées que nous venons de décrire. Ces dernières ne peuvent dès lors être directement assimilées à de la parure corporelle.

Le lot $\mathrm{n}^{\circ} 2$ a été subdivisé en trois sous-ensembles : le lot $\mathrm{n}^{\circ} 2 \mathrm{c}$ comprend deux valves de Laevicardium crassum encastrées et opposées (fig. 83). Là encore les charnières sont opposées et les perforations dirigées vers le haut, suggérant un mode de suspension analogue à celui que nous venons de décrire pour le lot $n^{\circ} 1$. Une nasse isolée se trouve au centre de la valve supérieure. Le lot $\mathrm{n}^{\circ} 2 \mathrm{~b}$ est composé d'une valve de Laevicardium crassum posée sur une valve d'huitre. Les traces d'usure observées autour de la perforation pratiquée dans la partie étroite de la valve d'huître suggèrent également la suspension libre de ces deux éléments. Les lots $n^{\circ} 2 b$ et $2 c$ recouvrent partiellement un troisième lot de coquilles (2a) composé d'un gros gastéropode et de quatre valves de Laevicardium crassum. Trois d'entre elles sont encastrées les unes dans les autres. Elles sont toutes trois disposées verticalement avec la charnière et la perforation orientées vers le haut, suggérant une suspension verticale. Ces trois coquilles sont recouvertes par une quatrième valve de Laevicardium crassum disposée horizontalement, avec la partie externe de la coquille tournée vers le haut. Contre la face concave de la première des trois valves verticales emboîtées, nous trouvons une coquille de Semicassis saburon. Elle est orientée verticalement, avec l'apex vers le bas. Cette coquille présente deux perforations, dont une présente des traces d'usure liées au passage d'un lien. La partie supérieure du canal siphonal, tournée vers le haut, présente une forte abrasion qui suppose un frottement contre un support. L'ensemble repose sur une dizaine de nasses disposées côte à côte qui pourraient avoir été fixées sur un même lien ou un sur même support. 
Fig. 83 - Lots $\mathrm{n}^{\circ} 1$ et 2 dans la structure 10.
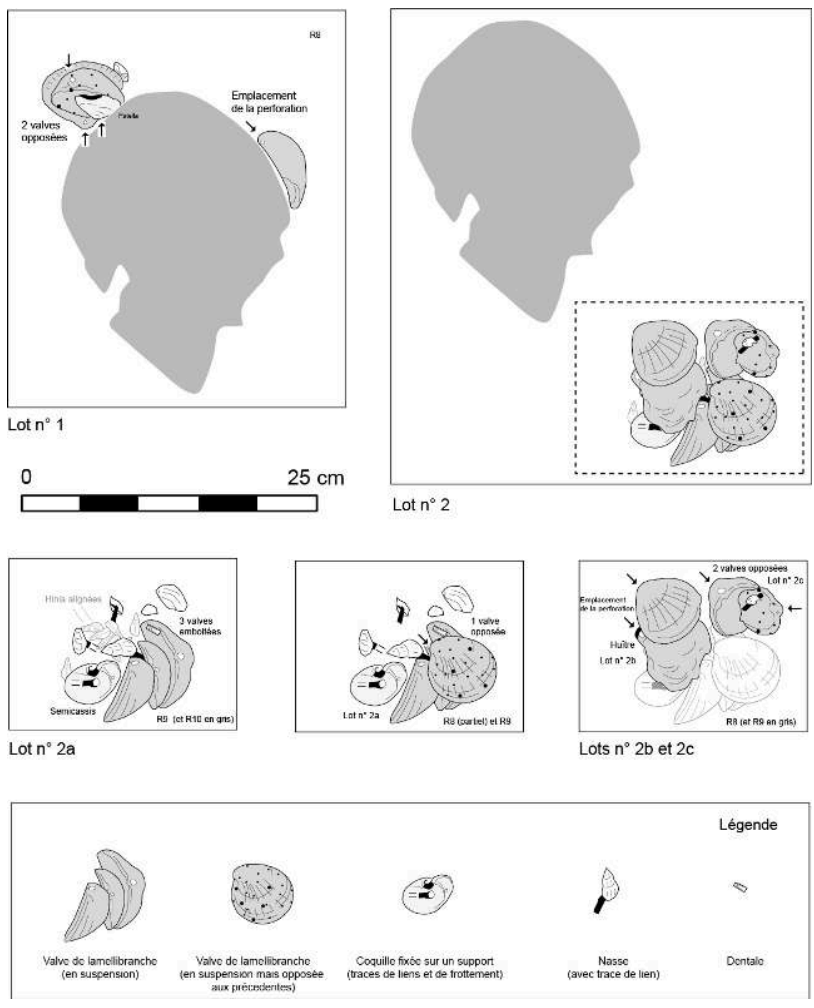

DAO de L. Laporte pour la figure, et de L. Quesnel pour le dessin de chaque pièce indépendamment, telles que reportées sur les relevés originaux dessinés par H. Duday avec également des annotations de P. Courtaud.

Le troisième lot est situé contre le menton du défunt (fig. 84). Il comprend trois valves encastrées les unes dans les autres. Les valves sont disposées verticalement, face externe vers le menton, avec les charnières et les perforations grossièrement dirigées vers le sud-ouest. Une valve de Glycymeris recouvre très légèrement cet ensemble, bien que décalée vers le nord. La partie interne de la coquille, disposée horizontalement, est tournée vers le haut. La charnière et la perforation sont dirigées vers le sud. Le tout est surmonté par une patelle, face externe vers le haut et percée au sommet, qui présente une plage d'abrasion latérale. Autant d'éléments que nous avons décrits presque à l'identique dans les lots $\mathrm{n}^{\circ} 1$ et 2 . Une valve de spisule et une coquille de patelle, décalées vers le sud-ouest, complètent l'ensemble. La valve de spisule est disposée verticalement. La perforation est orientée vers le haut. Sur chaque côté on observe des traces d'usure qui suggèrent un frottement contre un support. La seconde patelle présente également des traces d'usure latérale. Disposée avec la face externe vers le haut, elle présente la particularité d'être percée sur un côté et non pas au sommet comme les précédentes. 
Fig. 84 - Lots $n^{\circ} 3$ et 4 dans la structure 10.
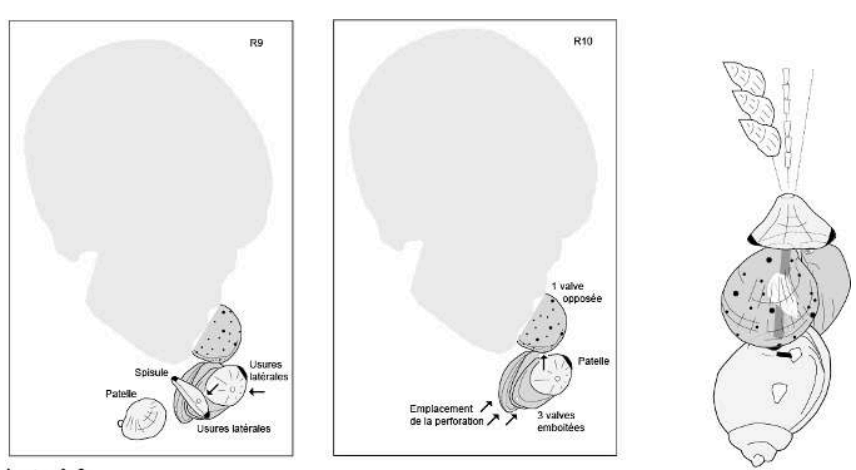

Lot $n^{\circ} 3$
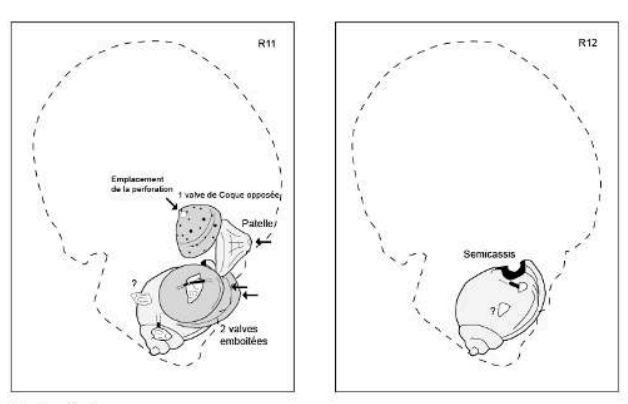

Lot $n^{\circ} 4$

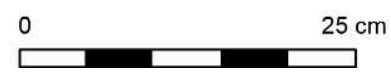

Construction de la figure par L. Laporte, DAO de L. Quesnel pour le dessin de chaque pièce indépendamment, telles que reportées sur les relevés originaux dessinés par $\mathrm{H}$. Duday avec également des annotations de P. Courtaud.

Le quatrième lot est situé sous la face du défunt (fig. 84). À la base il comprend une coquille de Semicassis saburon, disposée horizontalement. Les deux perforations sont dirigées vers le haut. L'une d'elles présente les traces d'usure liées au passage d'un lien, de même que la partie supérieure du canal siphonal. Elle est recouverte par deux valves horizontales dont la face interne et concave est orientée vers le haut. À l'intérieur de la valve supérieure, notons la présence d'une nasse isolée, dont les traces d'usure indiquent qu'elle fut fixée sur ou par un support en matière périssable. Une patelle couchée sur le côté surmonte localement cet ensemble. Elle présente une perforation sommitale et des traces d'usure latérales. Un peu plus au nord, une valve isolée de Cerastoderma est plantée verticalement, face externe vers le nord et perforation dirigée vers le bas.

On aura pu remarquer que chacun de ces différents lots présente des configurations homologues. À chaque fois, nous retrouvons une ou plusieurs valves emboîtées opposées à une valve isolée. Toutes ces valves étaient suspendues par ou sur plusieurs liens, souvent verticaux. À l'intérieur de la face concave de la plus interne de ces valves se trouve parfois une coquille de gastéropode, fixée sur un lien ou sur tout autre support. Il peut s'agir d'une grosse coquille de type Semicassis ou d'une petite nasse. L'ensemble est parfois coiffé par une patelle, percée au sommet et présentant de traces d'usure sur un ou deux bords opposés. Ce n'est donc certainement pas par hasard si l'on retrouve tout ou partie de chacun de ces éléments sur chaque lot individualisé. Tenter de proposer une reconstitution des objets correspondant parait bien périlleux; l'essentiel, c'est-à-dire toute la partie organique, ayant disparu. On peut toutefois 
remarquer qu'en suspension libre les valves opposées peuvent former comme une caisse de résonance, leur entrechoquement produisant un son caractéristique, un peu à la manière de clochettes, de castagnettes ou de maracas. La présence d'une patelle au sommet pourrait alors permettre de rassembler tous les liens nécessaires à la suspension des différentes pièces sous-jacentes, tout en assurant la cohésion de l'ensemble. Mais ce n'est là qu'une hypothèse parmi d'autres qu'il convient de prendre avec une extrême précaution.

Il nous reste à envisager maintenant le cas des pièces percées inscrites dans le volume corporel de l'adulte. C'était déjà le cas de la nasse et de la valve plaquées contre le crâne, sur le sommet de la tête. Peut-être ces coquilles étaient-elles fixées dans les cheveux. Dans les dix premiers centimètres du remplissage, on observe un semis assez lâche de nasses et de quelques rares dentales recouvrant tout le corps (fig. 85). Une valve de spisule est présente au niveau de la ceinture et une patelle se trouve sur le dessus de la cuisse droite. Dans les dix derniers centimètres du remplissage, une telle image n'est pas fondamentalement modifiée. Quelques pièces ont glissé à l'intérieur de la cage thoracique, alors que d'autres se trouvent coincées entre les os des membres droit et gauche, tant au niveau des membres supérieurs que des membres inférieurs. Un nombre encore plus limité de coquilles repose sur le fond de la fosse. La plupart de ces coquilles percées appartenaient sans doute à des éléments de parure corporelle dont il est difficile de déterminer la nature. Il n'est pas exclu que ce soit aussi le cas de quelques nasses situées sous la partie postérieure droite du crâne, à l'extérieur et contre l'effet de paroi cité plus haut. Aucune pièce similaire n'était posée symétriquement sur la partie postérieure gauche du même crâne. Au sein de cet ensemble, quatre nasses au moins ont été percées en suivant un schéma opératoire un peu particulier qui consiste à préparer la perforation en pratiquant une incision oblique, ou deux incisions parallèles délimitant deux bords opposés de la future perforation. Cette caractéristique n'a jamais été observée sur les autres pièces provenant d'autres secteurs de la même tombe. 
Fig. 85 - Coquilles étroitement associées au volume corporel de l'adulte déposé dans la structure 10 .
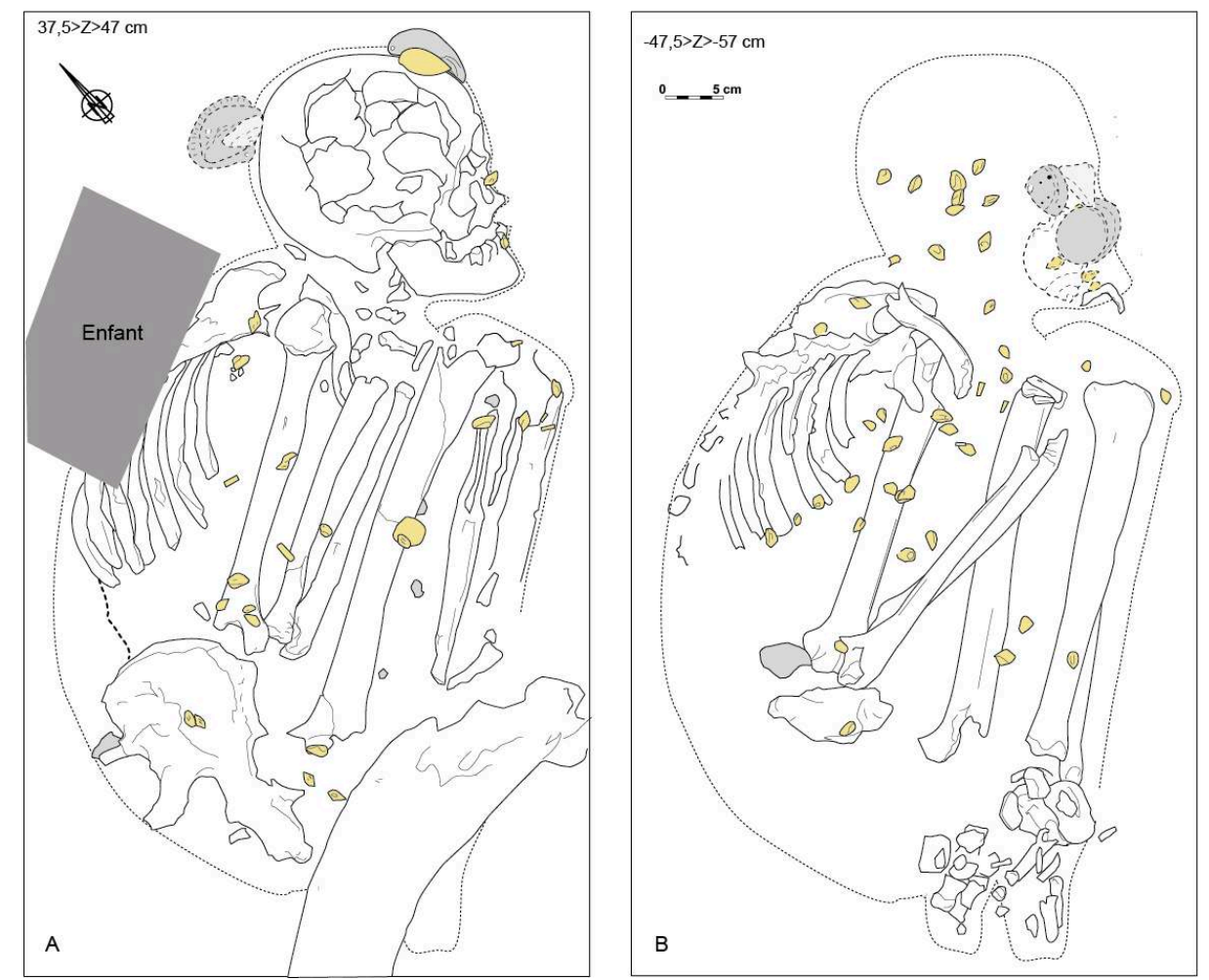

A. Tous les os sont en place; B. La moitié droite du corps a été démontée.

Construction de la figure par L. Laporte, DAO de L. Quesnel pour le dessin de chaque pièce indépendamment, telles que reportées sur les relevés originaux dessinés par H. Duday avec également des annotations de P. Courtaud.

Il est surtout intéressant de noter que les nasses étroitement associées aux volumes corporels de l'adulte présentent une usure nettement plus prononcée que toutes les autres de la même tombe (fig. 86A). Dans le premier cas, près des deux tiers des pièces concernées portent effectivement de tels stigmates. Ils sont même particulièrement accentués pour un tiers d'entre elles. En revanche, seules quelques rares traces d'usure ont été observées sur les pièces étroitement associées aux os d'enfant. Elles sont inexistantes pour les nasses fixées sur le contenant en matière périssable qui enveloppait ce dernier. L'usure du bord columellaire de la coquille constitue l'une des particularités des nasses recueillies dans la structure 10, et les distingue statistiquement de celles présentes dans la structure 7 , comme nous l'avons déjà montré. Elle suppose un mode de fixation particulier et original. Cette particularité se rencontre sur des pièces réparties dans toute la tombe. Elle est cependant, encore une fois, particulièrement accentuée sur celles qui sont étroitement associées au volume corporel de l'adulte, mais également dans ce cas, sur celles mêlées aux os d'enfant (fig. 86B). Sans qu'on puisse l'affirmer avec certitude - cf. vêtements portés successivement au même âge par les membres d'une fratrie, parfois étendue aux cousins, ou, à l'inverse, aux robes de mariées utilisées pendant un temps très court mais qui peuvent se transmettre sur plusieurs générations, aujourd'hui encore - il serait tentant d'imaginer que la parure correspondante a été portée plus ou moins longtemps en fonction de l'âge des individus concernés. Une telle hypothèse d'une transmission 
intergénérationnelle d'éléments de parure a aussi été proposée pour le gisement funéraire mésolithique de Große Ofnet en Allemagne (Rigaud 2013).

Fig. 86 - Degré d'usure des nasses percées recueillies dans la structure 10.
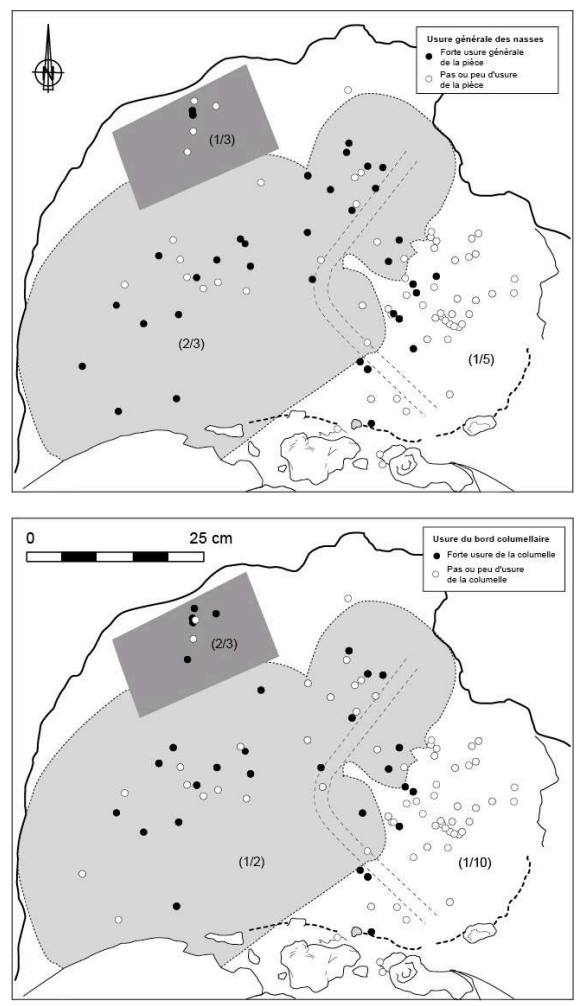

Les chiffres entre parenthèses indiquent la proportion de pièces fortement usées pour chaque ensemble.

DAO L. Laporte.

En définitive, la parure portée par l'adulte au moment de son inhumation comprend tout au plus une soixantaine de coquilles percées, essentiellement des nasses, quelques rares dentales, une valve de spisule et peut-être une patelle. Il faut peut-être ajouter à cela, en tant qu'éléments de parure corporelle, quelques dizaines de nasses étroitement enchevêtrées avec les ossements d'enfant déposés dans son dos. Dans tous les cas, cela ne représente qu'une petite partie des 435 coquilles percées déposées dans la tombe. La plupart d'entre elles, tout comme les canines percées, semblent plutôt correspondre à l'ornementation de divers objets en matière périssable accompagnant le défunt.

\section{Conclusions}

Une large part des coquilles recueillies lors de la fouille des sépultures de La Vergne semble plutôt correspondre au dépôt d'objets en matière périssable richement ornés, sans pour autant exclure, même parmi ces derniers, la présence de quelques parures corporelles ou de vêtements ainsi décorés. Seule la présence d'une ornementation composée ici de pièces en coquille permet parfois d'identifier l'existence de tels objets. Ils contribuent largement à la richesse et à la diversité spécifique des pièces recueillies dans chaque structure. C'est en particulier le cas des nombreux dentales qui caractérisent le spectre spécifique de la structure 3 comme des valves dans la 
structure 10. Pour autant, il nous est souvent impossible de reconstituer précisément la nature des objets décorés. Une simple visite de collections archéologiques pour des périodes plus récentes où les tissus comme les cuirs ont été préservés, et surtout de collections ethnographiques, suffisent à nous convaincre de la variété des objets, sacrés ou destinés à des fins utilitaires, susceptibles de porter une telle ornementation. La disposition des ornementations en coquille comme les traces de lien que portent ces derniers, suggèrent qu'ils furent fixés ou contenus dans des volumes de formes parfois très différentes. La présence de traces d'usure indique que certains au moins de ces objets ont été utilisés auparavant, en particulier ceux qui correspondent aux valves emboîtées de la structure 10. Mais on ne peut exclure pour autant que d'autres aient été confectionnés spécialement pour l'occasion. Il existe en fait autant de cas particuliers que de structurations spatiales observées dans les tombes de La Vergne. Il n'en reste pas moins que ces dépôts contribuent largement à la mise en scène du défunt dans sa tombe.

Un nombre de pièces plus restreint peut être assurément associé aux volumes corporels. Dans la structure 10, elles représentent moins du quart des coquilles percées. Toutes les parties du corps sont concernées, mais l'état de la documentation ne permet guère d'observer d'associations récurrentes. Du moins peut-on affirmer que le cou ou les extrémités des membres (colliers et bracelets) ne sont pas particulièrement privilégiés. De même, l'articulation des membres supérieurs ne fait l'objet d'aucun traitement spécifique. Dans deux cas sur quatre un objet de parure de grande dimension (patelle ou canine d'ours percée) est placé sous le genou gauche, alors qu'aucune observation similaire n'a été effectuée au niveau des genoux droits. Il serait prématuré d'en déduire un quelconque indice de latéralisation de la parure corporelle, a fortiori dans le cas d'individus placés sur le côté gauche en position contractée. La jambe gauche du grand enfant inhumé dans la structure 3 est associée à une parure abondante, mais on ne dispose d'aucune information symétrique pour sa jambe droite ni pour le reste du corps, irrémédiablement détruits. Dans ce cas les éléments du vêtement contribuent également à la diversité de la parure en coquille dans la tombe, notamment par la présence de coquilles de natices. On remarquera que les nasses correspondantes se distinguent par une taille largement supérieure à la moyenne. C'est également le cas de certaines des nasses recueillies au niveau du bassin des deux individus en connexion de la structure 7 et de la plupart de celles qui s'inscrivent dans le volume corporel de l'adulte inhumé dans la structure 10. Quelques coquilles ont été recueillies sur le torse ou dans la cage thoracique des cinq individus en connexion. Pour autant on ne sait pas toujours dans quelle mesure elles appartenaient initialement à des éléments de parure corporelle proprement dite ou si elles témoignent de la présence de liens voire d'une étoffe destinée à maintenir les membres supérieurs en position contractée. Un problème du même ordre se pose pour les objets de parure recueillis tout autour des ossements voire des corps des deux enfants inhumés respectivement dans les structures 7 et 10 . Quant à la parure de tête, elle reste très mal connue car quatre crânes sur cinq ont disparu. Une valve pourrait avoir été fixée dans les cheveux, sur la tête de l'adulte déposé dans la structure 10.

Existe-t-il un rapport entre l'ornementation des objets déposés dans la tombe et celle des individus inhumés ? Dans la structure 7, deux lots de pièces percées pourraient être mis en relation avec les deux sujets en connexion au pied desquels ils ont été disposés. C'est du moins ce que suggère l'abondance relative de dentales, récurrente dans un cas 
et pas dans l'autre. Parmi les objets identifiés, rien ne permet d'exclure la présence de quelques éléments de parure corporelle déposés séparément dans la tombe : on peut du moins se poser la question pour les lots de pièces percées comprenant des valves emboittées, déposés sous ou en avant de la face de l'adulte inhumé dans la structure 10 mais isolés par un effet de paroi, alors qu'un autre lot similaire plaqué contre l'arrière du crâne aurait pu être accroché dans les cheveux. Ces pièces portent quelques traces d'usure qui sans être très accentuées n'en sont pas moins assez généralisées, et l'une des techniques employées pour la préparation de la perforation artificielle des Semicassis saburon l'est aussi pour quelques nasses percées étroitement associées au volume corporel de cet individu - et seulement dans ces cas-là. Objets et pièces des vêtements ne semblent pas tous avoir été réalisés en même temps, ni peut-être par les mêmes personnes. Quelques nasses strictement associées au volume corporel de la femme adulte déposée dans la structure 3 se distinguent, comme dans la structure 10, par une technique de perforation originale. Si l'on ne peut pas affirmer que les objets richement ornés du dépôt funéraire ont été confectionnés pour l'occasion, du moins certaines parures strictement associées aux volumes corporels ont-elles été longuement portées au préalable. Une usure nettement plus accentuée des pièces percées associées à deux individus au moins, en témoigne. Faut-il en déduire qu'il s'agit là des pièces de vêtement habituellement portées de son vivant par le défunt? Ce n'est pas certain car, participant à la mise en scène des funérailles, ces éléments du costume peuvent ici prendre une signification différente de celle qui leur était attribuée dans le monde des vivants.

\section{Discussions}

Ce travail avait pour but de caractériser un ensemble de plus de 3000 coquilles percées de leur ramassage dans leurs lieux potentiels de découverte à leur dépôt avec les défunts. Ces coquilles ont été recueillies à l'occasion d'une fouille de sauvetage sur le cimetière du Mésolithique ancien de La Vergne. Cette première approche n'a pas épuisé les possibilités d'étude sur ce corpus exceptionnel tant par le nombre de pièces que par la finesse des observations effectuées sur le terrain. Une analyse plus détaillée des stigmates observés sur chaque pièce pourrait être maintenant envisagée, selon un principe qui part du général vers le particulier. La démarche inverse, souvent privilégiée, nous aurait sans doute noyés dans les détails. Les matières premières utilisées comme les techniques employées entrent dans la variabilité de celles attestées au moins dès le Paléolithique supérieur. Elle sera occasionnellement retrouvée sur des ensembles du Néolithique.

\section{Spectres fauniques}

170 Les principales caractéristiques de la collection de coquilles du site mésolithique de La Vergne ont été présentées. Au Mésolithique, le regroupement des sépultures en cimetières est observé (May 1986, p.10, Cauwe 2005). Seuls Téviec et Hoëdic sont comparables en France à La Vergne de par le regroupement de sépultures associées à des concentrations de coquilles percées. Les deux sites du sud de la Bretagne sont plus récents que celui de La Vergne de près de 3 millénaires avec des datations qui se distribuent autour de $5200-4500$ cal. BC pour Hoëdic et $5000-4900$ cal. BC pour Téviec (Marchand et Schulting 2019). D'autre part, Téviec et Hoëdic étaient sans doute 
plus proches des influences marines lors de leur fonctionnement que ne l'était La Vergne. En plus d'être des nécropoles, Téviec et Hoëdic sont également des amas coquilliers dans lesquels sont creusées les sépultures.

\section{Points communs aux nécropoles mésolithiques du littoral atlantique français}

La composition générale de la parure de ces 3 sites se ressemble beaucoup. En effet, pour ces trois sites, bien que des éléments d'autres natures soient présents, les coquilles marines en composent la majorité. On y observe également, entre autres, des craches de cerf (Jeunesse 1997, Rigaud 2011). Ces ornements ont aussi été découverts pour ces trois sites en association avec les squelettes.

Un autre point commun est l'utilisation, en majorité, d'espèces de petites dimensions (inférieures à deux centimètres; Taborin 1974). Il s'agit de la littorine obtuse Littorina obtusata associées à des 'porcelaines' ou cyprées Trivia monacha (Trivia europea dans Taborin 1974) pour Téviec et Hoëdic et des dentales associés aux nasses pour La Vergne. La composition binaire décrite pour ce dernier site se retrouve également. Pour Téviec, 3830 cyprées sont associées à 2960 littorines obtuses pour seulement 150 autres coquilles de diverses espèces (Rozoy 1978, p. 788). À Hoëdic, les littorines obtuses dominent avec 4391 coquilles décomptées pour 616 cyprées et 70 autres tests d'espèces variées (Dupont 2006). Parmi ces espèces minoritaires en nombre, on retrouve de grands bivalves dont certains sont communs à La Vergne comme l'amande de mer, l'huître plate, la bucarde de Norvège, mais aussi des espèces de plus petites dimensions comme la patelle, le murex, la nasse réticulée, le dentale, la coque et la natice (Taborin 1974, Dupont 2006). Parmi les espèces minoritaires de Téviec, la troisième position de la nasse réticulée mérite d'être soulignée avec 126 individus représentés.

Comme pour La Vergne, les coquilles majoritairement utilisées en tant que parures à Téviec et Hoëdic ne font pas partie du registre alimentaire de ces populations côtières (Dupont 2006). La présence des tombes de Téviec et Hoëdic dans un « kjokkenmödding » (Péquart et al. 1937) ou amas coquilliers, c'est-à-dire de déchets de cuisine (Rozoy 1978, p. 785), avait d'ailleurs permis aux fouilleurs de conclure que les coquilles servant dans l'alimentation sont peu nombreuses dans la parure, par rapport à celles dédaignées dans la nourriture (May 1986, Taborin 1974, p. 156). Comme nous l'avons souligné pour La Vergne (fig. 22), cette distinction est intéressante car elle montre que le choix fait par les populations mésolithiques n'est pas seulement guidé par l'abondance des coquilles sur la côte. Des choix d'ordre culturel sont également opérés.

D'autre part, la présence de mollusques subtidaux parmi la parure de Téviec et d'Hoëdic, ainsi que l'observation de stigmates liés à l'érosion marine, sur certains des exemplaires du musée de Carnac de Téviec et d'Hoëdic, montrent un comportement similaire à ce que nous avons décrit à La Vergne, à savoir, un ramassage de coquilles dans les laisses de haute mer. Cette activité est clairement différenciée de la quête de nourriture (Dupont 2019). Elle ne dépend pas des coefficients de marée et les espèces sélectionnées sont pour la plupart inférieures à $20 \mathrm{~mm}$. Or, les données en archéomalacologie le long du littoral atlantique français montrent que cette dimension, est souvent le minimum requis pour faire du coquillage un fruit de mer. La sélection d'espèces, en majorité, subtidales (pour le dentale), de bas niveaux de l'estran (pour les cyprées), inféodés aux algues (pour les littorines obtuses et les cyprées), vivant enfouies dans le sable (pour les nasses réticulées), de petites dimensions (pour ces quatre 
espèces) pour confectionner de la parure a également une autre conséquence. Ces quatre espèces sont rarement observées vivantes par les populations côtières. Ce fait peut ajouter une autre dimension à ces coquilles qui peuvent être ainsi porteuses de mystère et de croyance, voire de peur de l'inconnu. Cette distinction entre coquillages bons à manger et coquilles bonnes à confectionner des parures est intéressante. Elle semble se perpétuer au fil de la chronologie, ce qui permet d'appuyer l'idée d'un imaginaire entretenu par les fonds marins (Dupont 2019). Malgré une distance sans doute plus importante des influences marines de La Vergne au littoral, la richesse spécifique sélectionnée ne semble pas impactée. Cette dernière y est de 18 espèces, tandis qu'elle est de 17 à Hoëdic et de 12 à Téviec. C'est un autre point commun à ces sites séparés de plusieurs centaines de kilomètres et de plus de 3 millénaires. Si nous avons pu mettre en évidence à La Vergne l'importance des grandes coquilles dans la différenciation des sépultures, cette comparaison à cette échelle est difficile pour les deux sites bretons au vu des données spatiales disponibles (Boulestin 2016).

\section{Différence et accessibilité de la ressource le long du littoral atlantique français}

Le profil général de l'assemblage malacofaunique de La Vergne est proche des sites bretons par sa composition binaire et par la présence de parures majoritairement de petite dimension. Il s'en différencie cependant par les espèces identifiées. En effet, si cyprée et littorine obtuse représentent la majorité du corpus de ces sites, le dentale et la nasse réticulée sont légion à La Vergne. Cette différence peut s'expliquer par le faciès des littoraux les plus proches de ces sites. En effet, Téviec et Hoëdic sont proches de côtes rocheuses, tandis que La Vergne est proche d'une zone estuarienne sans doute ensablée voire en partie envasée. Ainsi, une seule différence marquante au sein de la composition même de la parure en coquilles marines peut s'expliquer par l'accessibilité à la matière première. D'autre part, le fait que ces trois sites étaient relativement proches du littoral au Mésolithique et que les espèces de mollusques marins identifiées étaient présentes sur le littoral nous oriente vers un ramassage de coquilles sur la côte au Mésolithique plutôt qu'une recherche de coquilles fossiles. Elles ont pu se faire par le biais d'échanges pour La Vergne sans qu'un ramassage direct par les hommes et les femmes de ce site ne soit exclu. En effet, en y regardant de près les données isotopiques, si les individus de ce groupe n'ont pas consommé massivement de ressources marines, une faible contribution de protéines d'origine marine a malgré tout été décelée (Schulting et al. 2008, p. 769) ce qui n'exclut donc pas le passage temporaire de ce groupe sur le littoral.

\section{Liens et connexions}

176 Malgré le faible nombre de sites, la liste des points communs énoncée pour la parure contraste avec la distance géographique, chronologique, la distance des sites au trait de côte et ce que cela implique au niveau des apports en ressources marines (Schulting 1996, 2008). La survivance des traditions portées par ces éléments de parure sousentend son importance pour les sociétés de chasseurs-cueilleurs qui ont fréquenté le littoral, même si le message véhiculé nous est inconnu. Il fait écho à d'autres travaux sur les liens et connections entre groupes humains mésolithiques « At the same time, the spread of non-'utilitarian', symbolically-charged items or ideas, such as geometric motifs and ornaments, possibly along with mythical narratives, is also evident on the basis of the presented data " (Boric et Cristiani 2016). 
177 À une échelle d'interprétation plus large encore, R. Newell et al. (1990) avaient mis en évidence deux grandes provinces géographiques concernant la répartition des pièces de parure mésolithique en Europe occidentale. L'une, où dominent les dents percées, est plutôt centrée sur l'Europe septentrionale et continentale. L'autre, plus méridionale, est centrée sur les pourtours nord-occidentaux de la Méditerranée, incluant également l'ensemble de la façade atlantique, où les pièces percées en coquillage dominent. Les travaux de S. Rigaud (2011) n'ont pas fondamentalement remis en cause cette distinction mais ils ont permis d'en affiner très largement les contours, désormais établis sur la base d'un corpus plus complet et d'une démarche statistique plus rigoureuse. Le site de La Vergne s'intègre parfaitement à ce schéma général où «la façade atlantique est notamment caractérisée au Mésolithique par la présence de Trivia sp., Nassarius sp., Laevicardium sp. Pecten sp., Littorina sp. et de craches de Cerf» (Rigaud 2011, p. 292, fig. 148).

\section{Actions anthropiques sur la matière}

Nous avons d'ores et déjà eu l'occasion de distinguer deux types d'actions anhropiques sur la matière (Laporte et Dupont 2019). Cette distinction est nettement mise en exergue par l'étude de tels objets dits "de parure». Les unes consistent à se réapproprier une unité naturelle, les autres à façonner une matière première. Le degré de façonnage de chaque type d'objet en coquille présent dans les sépultures de La Vergne sera détaillé, avant d'en détailler les modalités de ce façonnage.

\section{Degrés de façonnage}

$\mathrm{Au}$ Mésolithique, en Europe occidentale, les matières premières utilisées pour confectionner des parures font appel majoritairement au monde animal (Rigaud 2011). Parmi ces dernières, les éléments les mieux représentés, et de loin, sont ceux parmi les plus caractéristiques, qui permettent d'identifier l'animal même après sa mort (coquilles, dents...). Ces éléments n'ont alors fait l'objet que d'un façonnage limité qui n'altère pas la forme générale de la pièce, et rarement les éléments les plus caractéristiques permettant leur attribution spécifique. Cela est vrai en particulier pour la parure en coquillage, particulièrement bien représentée dans les sépultures mésolithiques du sud de l'Europe occidentale et sur sa façade atlantique. On retrouve de tels points communs dans le façonnage des objets de parure en coquillage sur le site de La Vergne, mais aussi dans les sépultures de Téviec et Hoëdic. Rappelons que si l'on tient compte seulement du nombre de pièces, ce qui peut porter à discussion, ces trois sites regroupent à eux seuls plus des $3 / 4$ des objets de parure mésolithiques répertoriés en Europe occidentale et continentale. Parmi les ensembles les plus significatifs, on observe presque toujours, comme pour le littoral atlantique français, la prédominance d'une ou deux espèces de coquilles de petite taille associées à des valves ou quelques grands gastéropodes. À titre d'exemple, les nérites dominent à Muge (Roche 1972), les cyprées (Trivia sp.) et les littorines obtuses à Téviec et Hoëdic, les nasses et dentales à La Vergne. Dans tous les cas, la forme de la coquille d'origine n'a généralement pas été affectée. Les tests sont utilisés entiers et l'unique façonnage réside dans la perforation, lorsque ce n'est pas un percement naturel qui a été utilisé.

180 Le terme de matière première n'est donc peut-être pas le plus judicieux, dans ce cas précis. Il s'agit plutôt d'une appropriation de témoins provenant du monde animal que 
d'une véritable transformation de la matière. Ces pièces, utilisées comme éléments de parure, témoignent de l'existence d'êtres vivants précisément identifiables. C'est également le cas des coquilles échouées sur l'estran qui furent sélectionnées pour être utilisées comme parure, puis enfouies sur le site de La Vergne. De la part de ces derniers chasseurs-cueilleurs il y a là une attitude très différente, comparée à celle des premiers agriculteurs de la façade atlantique. On serait plutôt tenté de voir en cela une attitude différente dans la manière d'appréhender et d'exploiter les ressources du monde naturel. À partir du Néolithique ancien, dans l'ouest de la France, les éléments intégrés dans la parure comptent de plus en plus d'objets manufacturés, dont la forme parfois purement conceptuelle ne rappelle en rien celle du support initial (Laporte et Dupont 2019). Peu à peu, les matières osseuses et les coquillages seront traités de plus en plus souvent comme une matière première inerte, parmi d'autres principalement issues du monde minéral.

\section{Modes de façonnage}

181 Lorsqu'il n'était pas possible de tirer parti des perforations que l'on rencontre naturellement, une perforation a été pratiquée. Dans ce cas, mais dans ce cas seulement, des techniques diversifiées sont employées pour un même type d'objet de parure. Sur les nasses qui dominent largement en nombre de pièces l'assemblage de La Vergne, les perforations d'origine anthropique sont cependant très fréquentes. Les parures en dentales sont aussi très abondantes dans le corpus étudié dont elles constituent près du tiers des effectifs. Ces coquilles de forme tubulaire ont été comparées à des référentiels comme cela a pu être fait sur d'autres sites archéologiques (Vanhaeren et d'Errico 2001, Rigaud 2014, Perlès 2018). Elles ont été façonnées par segmentation des extrémités distales et proximales de la coquille. Seule la partie médiane a été utilisée. L'aspect des extrémités est d'ailleurs différent de ce qui a pu être observé sur des dentales échoués sur la plage (Perlès 2018).

Presque toutes les valves utilisées comme objet de parure sont des coquilles échouées sur l'estran et sélectionnées du fait de la présence d'une perforation naturelle près de la charnière. Il existe cependant quelques cas où la perforation a été obtenue par une abrasion artificielle du crochet. C'est également le cas des valves recueillies dans les sépultures mésolithiques de Téviec et Hoëdic, qui sont percées au niveau du crochet ou de l'umbo (Péquart et al. 1937, p. 95). Seule une valve plate d'huître fait exception dans le cadre du site de La Vergne, la coquille étant percée au niveau de son bord dorsal. La perforation des pendeloques généralement pratiquée sur la partie avant et bombée de la coquille a souvent été associée à un mode de suspension et de présentation des coquilles (Barge-Mahieu et Taborin 1991, p.4) ainsi qu'à une stabilité à plat de l'ornement suspendu (Péquart et al. 1937, p. 95).

183 Parmi les coquilles de gastéropodes percées il convient de distinguer les nasses qui constituent près des deux tiers de l'ensemble du corpus (tabl. 4). Il s'agit pour la plupart d'exemplaires échoués et certaines présentaient naturellement une ou plusieurs perforations. Lorsque ce n'était pas le cas, ou lorsque les orifices naturels n'étaient pas situés à l'emplacement souhaité, la perforation a été effectuée par percussion. Aucune trace résultant d'une percussion lancée n'a été observée sur les exemplaires de La Vergne. On peut discuter de la face, interne ou externe, à partir de laquelle cette percussion a été réalisée. En revanche, les perforations effectuées sur les Euspira sp. de la structure 3 ont été réalisées par le biais d'une technique très différente qui fait appel 
à l'usage d'un foret rotatif. Cette même technique n'a été observée de façon certaine que sur une seule nasse provenant de la structure 7.

Tabl. 4 - Principales techniques employées (fréquemment en noir, plus rarement en gris) pour la perforation des coquilles.

\begin{tabular}{|l|c|c|c|c|c|c|c|c|}
\hline & Tritia & $\begin{array}{c}\text { Theodoxu } \\
\text { fluviatilis }\end{array}$ & Ocenebra & Semicassis & Euspira & Ostrea & $\begin{array}{c}\text { Autres } \\
\text { Bivalves }\end{array}$ & Patella \\
\hline
\end{tabular}
\begin{tabular}{|l|l|l|l|}
\hline Réparation de la perforation de la surface \\
Incisions parallèles \\
Rainurage \\
Abrasion
\end{tabular}
$\begin{aligned} & \text { Perforation } \\
& \begin{array}{|l}\text { Percussion } \\
\text { Foret rotatif }\end{array} \\
& \text { Reprise de la perforation }\end{aligned}$
\begin{tabular}{|l}
\hline ? \\
\hline
\end{tabular}

L'emplacement de la perforation à l'aplomb du péristome a parfois été associé à un percement du test par l'intérieur de la coquille. C'est l'hypothèse qu'a proposée Y. Taborin (1974) qui envisage l'utilisation d'un percuteur ou d'une pointe en silex par percussion indirecte ou interposée, à partir de la face interne concave (Barge-Mahieu et Taborin 1991, p. 7). L'orifice obtenu présente alors des bords irréguliers, plus rarement circulaires, et un profil conique. La partie large du cône se trouve sur la face convexe. Des ébréchures peuvent se lire sur le pourtour de la face externe (Barge 1982, p. 76). Cette méthode aurait été largement utilisée par les groupes épipaléolithiques et mésolithiques par introduction d'une pointe dans le péristome. C'est en particulier l'hypothèse proposée pour le percement des cyprées, des nasses et des littorines provenant du site de Téviec et Hoëdic (Péquart et al. 1937, p. 80). En revanche, sur la grande majorité des pièces examinées de La Vergne, les écaillures résultant de cette opération ont été observées sur la face interne de la lèvre de la perforation. Cela serait plutôt en accord avec une perforation obtenue par percussion posée sur la face externe et concave de la coquille. On comprend dès lors l'utilité d'une préparation préalable de cette surface convexe chez les gastéropodes turbinés.

Une préparation préalable des surfaces par abrasion ou par incision a parfois pu être détectée sur quelques nasses de La Vergne. C'est cette dernière opération qui semble présenter la plus grande variabilité. La technique qui consiste à préparer la perforation par deux encoches parallèles, en délimitant le contour, a également été utilisée sur l'une des deux grandes coquilles de Semicassis sp. et sur l'unique exemplaire de murex percé. La préparation de la perforation par raclage de la surface du test ou par rainurage, n'a été observée que sur quelques nasses. Dans presque tous les cas le contour de la perforation semble avoir été ensuite régularisé à l'aide d'un instrument tranchant.

Même lorsque la coquille porte plusieurs orifices naturels ou artificiels, une seule perforation semble avoir été utilisée. Elle est située majoritairement en E2, c'est-à-dire à proximité du labre tout en laissant un pont suffisamment large entre les deux pour éviter une cassure de la coquille à cet endroit. C'est là, sans doute pour des raisons techniques, un point commun avec la plupart des gastéropodes turbinés portant une perforation et recueillis dans les sépultures préhistoriques en France (Taborin 1974, 
May 1986, Taborin 1993). À La Vergne, seules les coquilles de grands gastéropodes, tels les deux Semicassis saburon, présentent deux perforations alignées le long de l'axe principal de la coquille. En cela, elles rappellent les nombreuses coquilles de cyprées biforées provenant des sépultures mésolithiques de Téviec et Hoëdic, bien que ces dernières soient de taille beaucoup plus petite. Les gastéropodes coniques représentés uniquement par les patelles sont percés soit à l'apex pour neuf d'entre elles soit au niveau $\mathrm{du}$ bord antérieur pour deux exemplaires. La perforation sommitale est également le cas le plus fréquent sur les patelles provenant des sépultures mésolithiques bretonnes (May 1986).

Tous ces éléments, outre qu'ils concourent à une histoire des techniques, ne prennent tout leur sens que lorsqu'ils peuvent être confrontés par ensembles cohérents; ce qui nous amène maintenant à considérer la place des objets dans la tombe.

\section{Place des objets dans la tombe}

Au sein de la partie précédente, la très grande qualité de l'enregistrement effectué à l'occasion de la fouille de ces sépultures de La Vergne, nous a permis de discuter de la place dans la tombe de chacun de ces objets en coquille marine. Il s'agissait notamment de distinguer celles qui participaient à l'ornementation de différentes pièces de vêtement, comme de la parure corporelle propre à chaque individu, de celles qui décoraient également d'autres types d'objets en matière périssable par ailleurs déposés dans la tombe. Nous avons vu que ce n'est pas toujours un exercice très aisé. Bien entendu, la reconstitution de la parure sera facilitée dans le cas d'une sépulture individuelle en pleine terre, dans un espace colmaté. Elle sera d'autant plus lisible que le défunt est en position allongée. C'est notamment le cas de la sépulture I de Soungir, en Russie au Paléolithique supérieur (Trinkaus, Buzhilova 2018), célèbre par la richesse et le caractère élaboré des 3500 pièces osseuses percées cousues sur un vêtement comprenant notamment des chausses, un pantalon et une veste.

En revanche, l'abondante parure provenant principalement de la couche $4 \mathrm{c}$ de l'aven des Iboussières à Malataverne (Drôme) ne nous sera pas d'une grande utilité dans le cadre de cette discussion, bien que beaucoup plus proche géographiquement et chronologiquement des sépultures de La Vergne. Elle comprend pourtant pas moins de 1112 dentales, 14 valves, 3 cyclopes, 1 Trivia europaea (d'origine atlantique), 280 dents percées, 2 pendeloques en os et 4 galets percés, associés aux restes osseux d'au moins 8 personnes. Malheureusement, l'essentiel de ce mobilier provient d'une désobstruction un peu brutale puis d'un tamisage à l'eau des sédiments correspondants (d'Errico et Vanhaeren 2000). En Europe occidentale, parmi les fouilles anciennes, rares sont également celles qui livrent des informations suffisamment précises concernant la place des objets percés dans la sépulture. La description de D. Peyrony quant à la place des quelques 1100 pièces de parure en coquillage - dont 900 dentales - sur le corps de l'enfant de La Madeleine, est bien trop laconique, tout comme le schéma publié (Capitan et Peyrony 1928, Vanhaeren et d'Errico 2001).

\section{Colliers, bracelets, parures de tête et pièces de vêtement}

190 Au XIX e siècle, comme à l'aube du siècle suivant, grains de colliers enfilés sur un lien ou noués dans les cheveux semblaient constituer les parures les plus conformes à l'image que l'on se faisait d'un Homme " primitif », conception alors largement étendue depuis 
les temps les plus reculés jusqu'à l'observation ethnographique de populations contemporaines. C'est aussi ce qui nous vaut, encore aujourd'hui, d'observer tant de colliers dans les vitrines de nos musées, pour les différentes périodes de la préhistoire. Si beaucoup de ces présentations résultent de présupposés obsolètes, il en est quelques autres cependant qui furent le fruit d'observations extrêmement précises.

191 Le compte rendu des fouilles en laboratoire effectuées par le Dr Verneau sur les squelettes recueillis dans les grottes de Grimaldi, est de ce point de vue exemplaire pour l'époque. Pour l'un d'eux il note : «En dégageant le squelette, qui avait fortement souffert avant mon arrivée, j'ai remarqué qu'il était accompagné de nombreux objets de parure. J'ai noté soigneusement la position qu'occupait chacun d'eux et j'ai pu me rendre un compte exact de la façon dont ils étaient disposés. » (Verneau 1906, p. 54). La sépulture inférieure de la Grotte des Enfants à Grimaldi contenait deux squelettes en connexion, étroitement imbriqués avec les membres en position très fortement contractée. Une telle disposition n'est d'ailleurs pas très différente de celle observée dans certaines tombes de La Vergne. «Dès que le squelette de la vieille femme fut un peu dégagé, on remarqua un bracelet composé de nasses percées au-dessus du poignet gauche. Plus tard on rencontra un certain nombre des mêmes coquilles au-dessous du tibia, et on pouvait croire alors qu'elles provenaient d'une sorte de jambière ; mais au mois de février 1905, j'ai achevé le dégagement des membres supérieurs et inférieurs gauches de ce sujet et j'ai découvert, immédiatement au-dessus de l'extrémité inférieure de l'humérus, deux rangées de nasses encore appliquées contre l'os du bras, auquel elles étaient fixées par une gangue solide. Étant donné la position du cadavre, il est évident que les nasses isolées recueillies antérieurement au-dessous du tibia provenaient du bracelet dont j'ai rencontré les débris en place. » (ibid., p. 29-30). Il s'agit là d'une démarche qui sur le principe n'est pas très éloignée de celle que nous avons tenté d'appliquer a posteriori pour le corpus de La Vergne. Les éléments de la démonstration sont parfois étayés par quelques photographies: il faut toutefois se méfier de la position de la parure sur la photographie publiée représentant la sépulture double dont nous venons de parler, qui est ici visiblement reconstituée pour une présentation au public.

En revanche, la photo du squelette de la Grotte voisine du Cavillon montre clairement une parure de tête encore en place. "L'homme de la Grotte du Cavillon avait la tête couverte de nasses et de vingt-deux canines de cerf percées; le tout devait constituer une sorte de résille analogue à celle que portent encore quelques femmes du peuple ou quelques pêcheurs de certaines contrées d'Italie.» (ibid., p.31). La référence ethnographique a ici le mérite d'être explicite pour une reconstitution de cette parure de tête sous la forme d'une résille, hypothèse qui deviendra florissante dans la littérature des décennies suivantes et parfois jusqu'à nos jours encore. Ces hypothèses sont parfois étayées par des observations précises, comme ici à propos de l'un des individus enterrés à Barma Grande : «En certains points s'était déposée une argile qui, en se mélangeant avec la couche de fer oligiste dans laquelle gisait le cadavre, avait formé une sorte de chape aux objets et les avait conservés dans leur position primitive. C'est grâce à cette circonstance qu'il m'a été permis de reconnaître l'agencement d'un joli collier composé de vertèbres de poisson, de nasses et de canines de cerf percées. Les vertèbres étaient disposées en deux rangées parallèles... » (ibid., p. 34).

La même particularité, associée à l'étude des traces d'usure, permettra cent ans plus tard à M. Vanhaeren (2002) de proposer une reconstitution de la pièce de vêtement 
correspondant au millier de cyclopes recouvrant le ventre et les cuisses des deux très jeunes individus découverts par E. Rivière dans la Grotte des Enfants. Des fouilles de plus en plus précises conduisent aujourd'hui à multiplier de tels exemples : la place de chaque objet dans la tombe peut alors être discutée même dans le cas de réductions de corps extrêmement poussées (Bugajska et Guminski 2016). Pour ne prendre que les parures de tête, si ces dernières sont désormais documentées de façon beaucoup plus précise (Butrimas 2016, p. 210, fig. 31), leur restitution débouche parfois aussi sur des représentations de plus en plus exubérantes (Petersen 2016, p.122, fig. 18b), pour la période du Mésolithique. Il n'est alors plus guère de freins pour mobiliser de tels objets dans la restitution de larges pièces du vêtement (voir aussi Boric et al. 2014, Boric 2016, Ghelen 2016, p. 841, fig. 15).

\section{Berceaux, et autres objets décorés déposés dans la tombe}

Contrairement au cas précédent, les propositions quant à la présence d'objets richement ornés qui furent déposés dans la tombe indépendamment de la parure corporelle, restent encore assez limitées pour la période préhistorique. Nous prendrons ici pour seuls exemples quelques sépultures mésolithiques du nord de l'Europe, en particulier celles provenant des cimetières de Bogebakken à Vedbaek et de Dragsholm au Danemark (Albrethsen et Petersen 1977). À Bogebakken, les 15 sépultures individuelles ne présentent généralement pas de parure corporelle ; seule l'une d'entre elles a livré cinq dents percées. Elles sont en revanche abondantes dans deux des trois sépultures multiples de ce cimetière. Avec un total de 50 pièces dans un cas et plus de 240 pièces dans l'autre, la quantité d'éléments de parure recueillis n'est pas directement proportionnel au nombre de personnes inhumées dans chaque tombe. Pour la préhistoire d'autres continents, nous avons eu déjà l'occasion de nous interroger sur les significations possibles de telles disparités (Laporte 2014). En Europe, elles sont particulièrement sensibles dans le cas des sépultures multiples de l'Epipaléolithique et du Mésolithique.

195 Au moment de leurs découvertes, les cinquante canines percées présentes au niveau du cou et sur la poitrine de l'un des deux adultes de la tombe 19 de Bogebakken semblaient alors correspondre, sans guère de doute, à des éléments de parure corporelle. C'était aussi le cas des nombreuses pièces percées recueillies au niveau de l'un des bras, dans et sous le bassin des deux femmes inhumées dans la tombe de Dragsholm. Une telle interprétation était cependant plus difficile à soutenir en ce qui concerne la tombe 8 de Bogebakken. Il s'agit de la sépulture d'une femme adulte déposée sur le dos et d'un enfant inhumé sur son côté droit, à la hauteur du torse de l'individu précédent. L'essentiel des pièces de parure est ici concentré au-dessus de l'épaule droite et contre la tête de l'adulte. Cet ensemble de 190 canines percées passe sous le crâne (comme cela a été observé par ailleurs à Téviec et Hoëdic pour des lots de coquilles). Il repose donc sur le fond de la fosse et ne peut guère être assimilé à une pièce du vêtement qu'aurait porté ce personnage lors de ses funérailles. Pourtant, une distinction entre parures masculines et féminines semble avoir été presque systématiquement remarquée dans ces tombes danoises. Une proposition du même ordre avait été effectuée par Y. Taborin (1974) pour les sépultures de Téviec et Hoëdic. Les auteurs danois ont d'abord interprété ce lot de parures comme appartenant à une pièce de vêtement repliée, servant d'oreiller sous la tête du défunt. On ne pouvait exclure pourtant qu'il s'agisse d'un tout autre type d'objet. 
Une récente mise au point, publiée quarante ans plus tard, éclaire désormais ces découvertes sous un jour un peu différent. L'hypothèse désormais privilégiée est celle d'un berceau utilisé pour le transport des enfants sur le dos de leur mère, au sein de populations de chasseurs- cueilleurs à forte mobilité. Cette hypothèse est ici argumentée sur la base de comparaisons avec des objets provenant de différentes collections ethnographiques, sur au moins trois continents distincts (Petersen 2016, p.118). Elle avait déjà été proposée pour la période antérieure du Paléolithique supérieur (Einwögerer et al. 2006). Elle est ici étendue à d'autres sépultures mésolithiques danoises où de telles concentrations de canines percées sont situées à proximité du corps d'un enfant en bas âge, comme pour la tombe 19 de Bogebakken déjà citée. Les dents percées sont alors interprétées comme autant d'amulettes prophylactiques, afin de protéger l'enfant du mauvais sort. Dès lors, l'abondance de tels objets dits de " parure » dans la tombe ne pourrait pas être mobilisée dans un discours concernant la différenciation sociale des adultes.

197 L'association directe entre certaines concentrations de coquilles percées et le corps, ou les ossements, de jeunes enfants est aussi un cas de figure que nous avons rencontré dans les tombes de La Vergne. Il est toutefois une hypothèse complémentaire, également attesté par maints exemples ethnographiques concernant les populations de chasseurs-cueilleurs, qui s'accorderait encore un peu plus avec nos propres observations de contenants décorés à la taille réduite également associés à quelques ossements compactés ou aux restes d'une crémation; celle d'un enterrement différé des jeunes défunts, transportés par leur mère dans l'attente du décès et de l'inhumation d'un individu adulte de ce même groupe (Boulestin 2016, p. 273). À la suite des travaux d'A. Testart (2004) qui s'interrogeait sur la fréquence des sépultures doubles ou multiples de la Préhistoire récente, B. Boulestin (2016, p. 269) introduit cet élément comme une alternative aux débats sur les pratiques de morts sacrificielles ou de morts d'accompagnements au sein des populations de chasseurs-cueilleurs.

Les exemples précédents partent tous de l'idée d'une liaison directe qui serait à établir entre le corps d'un individu humain et les volumes d'un objet en matière périssable richement décoré (fig. 87). À La Vergne, cette liaison peut être étendue à certaines parties du corps d'un animal, comme pour les cornes d'aurochs déposées dans la tombe 10 (fig. 88). Les exemples ethnographiques comme les représentations de bovinés aux corps et aux cornes richement parés sont extrêmement nombreux, mais plutôt pour des animaux domestiques et notamment à l'occasion de leur sacrifice. 
Fig. 87 - Structuration spatiale de la parure dans la tombe 7 de La Vergne, principales hypothèses envisagées.

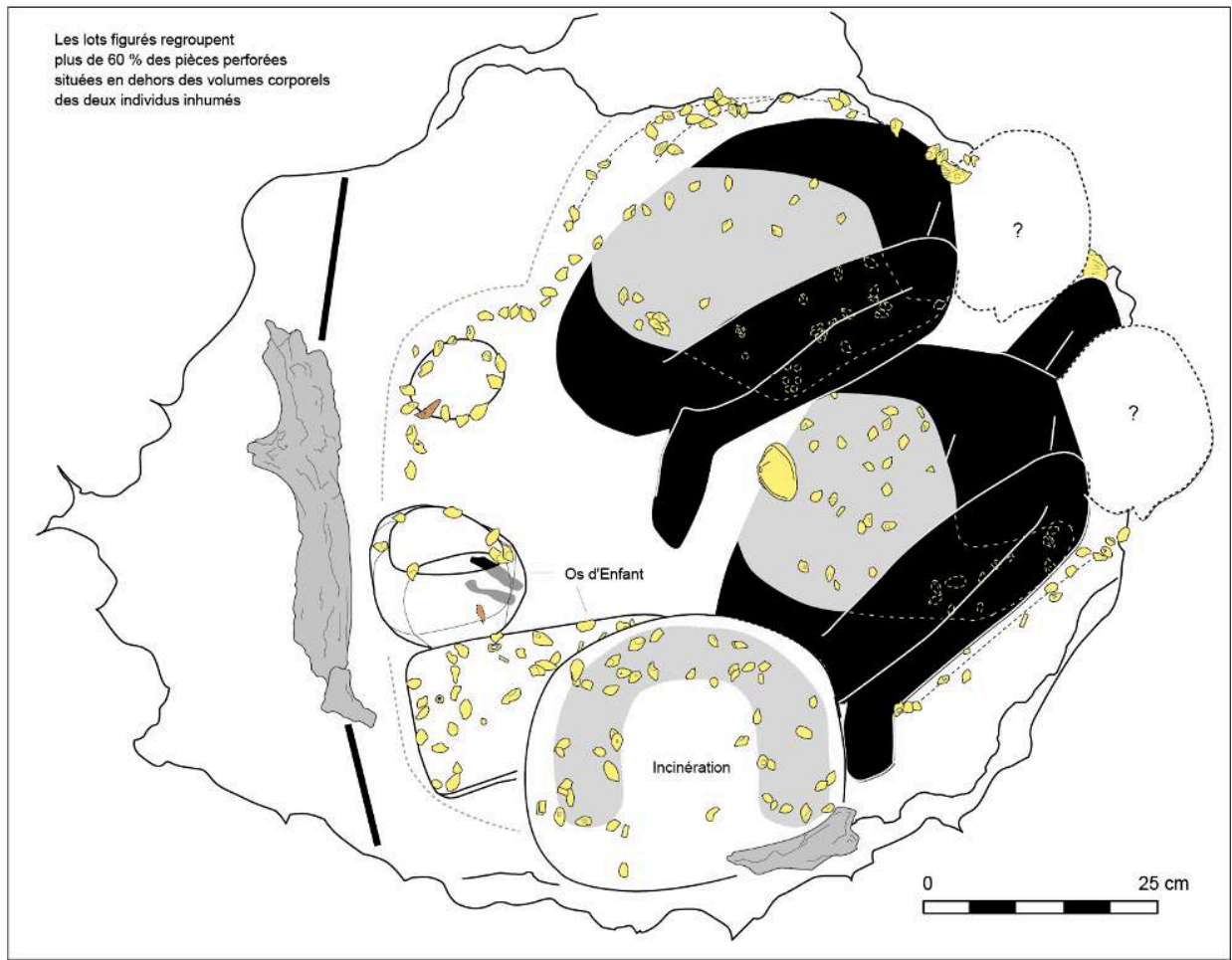

Construction de la figure L. Laporte ; DAO L. Quesnel pour le dessin de chaque pièce indépendamment, telles que reportées sur les relevés de fouilles originaux dessinés par H. Duday, avec également des annotations de P. Courtaud. 
Fig. 88 - Structuration spatiale de la parure dans la tombe 10 de La Vergne, principales hypothèses envisagées.

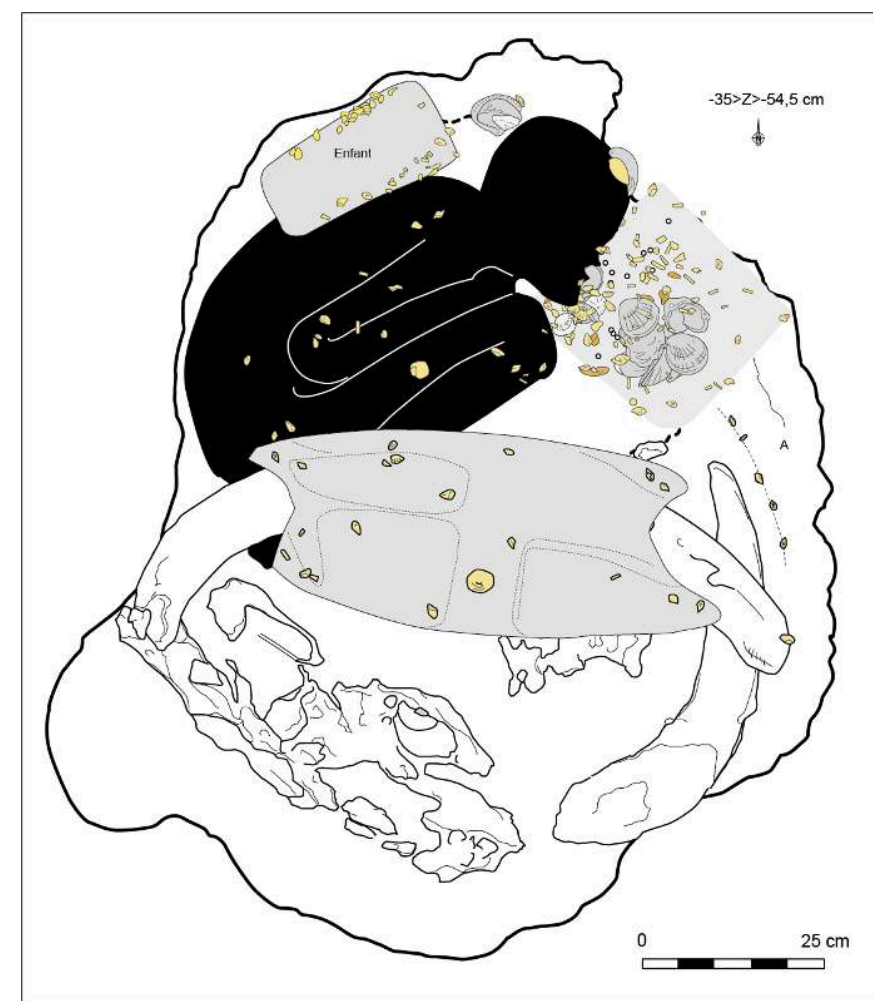

Construction de la figure L. Laporte ; DAO L. Quesnel pour le dessin de chaque pièce indépendamment, telles que reportées sur les relevés de fouilles originaux dessinés par H. Duday, avec également des annotations de P. Courtaud.

D'autres objets déposés dans les tombes mésolithiques de La Vergne sont encore plus difficiles à identifier. Nous avons seulement fait remarquer que l'entrechoquement de valves opposées de bivalves rassemblées par un même lien pourrait avoir fonctionné comme autant de grelots, un peu à la manière de ces bracelets de chevilles dont se parent danseurs et danseuses lors de cérémonies traditionnelles. À l'avenir, l'analyse des microtraces sur coquilles (Bar-Yosef Mayer et al. 2020) permettrait peut-être de savoir la nature du contact des coquilles entre elles (frottement, entrechoquement). Dans ce cas précis, et parmi bien d'autres possibilités, on ne peut donc pas totalement exclure qu'il s'agisse d'un ornement corporel, ou d'une pièce de vêtement, sur laquelle reposerait la tête du défunt. Quoi qu'il en soit, ce n'est assurément pas le cas le plus fréquent au sein des sépultures de La Vergne où nous avons vu que tant d'autres dessinent par exemple la forme de divers contenants, ou enveloppes. D'une façon plus générale, l'existence dans les tombes préhistoriques d'objets ornés en matière périssable qui soient totalement indépendants des volumes corporels fut rarement soulignée, peut-être aussi du fait du poids symbolique que représentent à nos yeux de tels vestiges conçus comme indissociables de la mort d'un être humain. Ils ne seront véritablement pris en compte que pour des périodes beaucoup plus récentes, comme pour les tombes égyptiennes par exemple, notamment lorsque les conditions de conservation ou le caractère pérenne du support à tant d'ornementation rendront leur identification incontestable. 


\section{Le cas particulier des sépultures de Téviec et Hoëdic, en Bretagne}

200 années 1930 sur les côtes méridionales de la Bretagne, restent des éléments de comparaison incontournables pour notre étude, de par le nombre des objets dits de parure qui leur sont associées. L'analyse détaillée de chacune des pièces de cette collection avait été intégrée à la thèse soutenue par Y. Taborin, dans les années 1970 (Taborin 1971). Des révisions plus récentes de l'ensemble de la documentation disponible sur ces sépultures ont été proposées (Schulting et al. 2008, Dupont 2006, Dupont et al. 2009, Boulestin 2016). Nous nous attacherons plutôt ici à la place de la parure dans chaque tombe sur la base des descriptions que nous ont léguées les fouilleurs, et l'observation de quelques photographies.

201 Le texte de ces descriptions est évidemment empreint des présupposés de l'époque. Lors de la découverte des sépultures épipaléolithiques de Grimaldi, en 1906, le Dr Verneau ne pouvait guère imaginer que des pagnes au niveau du bassin, des colliers sur la poitrine, quelques bracelets autour des membres, des résilles ou des couronnes sur la tête. Ce n'est peut-être pas un hasard si nous retrouvons presque exactement les mêmes termes appliqués dans la description de la parure des sépultures mésolithiques de Téviec et Hoëdic, quelques années plus tard, sous la plume des époux Péquart qui fréquentaient alors cette même équipe de l'Institut de Paléontologie Humaine.

202 Dans la tombe $\mathrm{H}$ du cimetière d'Hoëdic, les époux Péquart décrivent ainsi le corps d'une femme adulte qui gît «légèrement penchée sur le flanc droit. La tête légèrement inclinée vers la poitrine avec un air de recueillement qu'accentue encore le geste de deux mains jointes à la base du cou et dont les doigts se crispent sur un Cardium percé. Nul doute qu'il s'agisse d'une pendeloque constituant la pièce principale d'un collier composé de littorines dont nous retrouvons certains éléments en place, sur la poitrine et les restes éparpillés dans le bassin" (Péquart et Péquart 1954, p. 44). Outre le romantisme de la narration, on ne manquera pas d'être intrigué par la longueur du collier ainsi reconstitué, qui partirait du cou pour se terminer dans l'entrejambe.

Un peu plus loin, la sépulture $\mathrm{K}$ était à l'origine celle d'un homme adulte, déposé sur le dos. La parure comprenait plusieurs centaines de gastéropodes de petite taille, 3 valves et 2 boucles de raie. Elle consistait en « bracelets dont les éléments sont encore en place à chaque poignet, collier dont on retrouve les perles autour du cou et sur le sternum. Par ailleurs l'existence d'une résille coiffant la tête du mort parait plus que probable, à en juger par le nombre de littorines percées accumulées derrière et sous le crâne, comme nous l'avons constaté après enlèvement de celui-ci. » (ibid., p. 57). On notera ici, comme dans l'exemple précédent, l'absence de coquilles percées disposées sur la tête du défunt, ces dernières étant plutôt situées en dessous et autour du crâne. Un examen détaillé de la documentation suggère désormais aux anthropologues une décomposition du corps en espace colmaté. L'hypothèse d'une résille enserrant la tête du défunt peut donc être écartée.

204 La sépulture J recelait les restes d'une femme adulte, allongée sur le dos, jambes fortement contractées vers le haut, avec le squelette d'un enfant sur le ventre. « Outre les 134 cyprées et 604 littorines percées, grains de colliers et bracelets épars un peu partout sur le squelette - et jusqu'auprès de la cheville gauche (restes probables d'un anneau de jambe) - nous voyons, enserrant étroitement le cou de l'inhumée, 25 valves de grande taille, encore en place, les unes à côté des autres dans un ordre parfait. En 
plus de ce collier imposant, et comme pour ajouter à l'esthétique du spectacle, deux ormeaux (Haliotis tuberculata) posés de part et d'autre du cou sur chacune des clavicules, miroitent encore à nos yeux de toutes leurs irisations nacrées. » (ibid., p. 54). Les deux ormeaux recueillis sur les clavicules pourraient effectivement faire partie d'un élément $\mathrm{du}$ vêtement. Mais retenons surtout la présence de nombreuses valves près de la tête du défunt, comme pour la tombe 10 de La Vergne où elles correspondent à des objets distincts de la parure corporelle proprement dite.

Les descriptions des sépultures de Téviec sont encore plus laconiques. Elles suscitent des interrogations du même ordre. La sépulture $\mathrm{E}$ contenait les restes de deux individus. " Le plus grand des sujets était assis face à l'est, le buste adossé à la paroi de la fosse et les jambes fortement repliées à gauche. Entre ses bras et allongé en travers de son corps, reposait un enfant dont le crâne se trouvait niché dans l'angle de son coude gauche et les petits pieds emprisonnés dans sa main droite, légèrement refermée. Entre les doigts de cette main était venue s'insérer une pendeloque (patelle percée) provenant très probablement d'un bracelet de bras ou de poignet. Le mort en effet était paré de "bijoux" composés de coquilles percées (1 500 littorines, nasses et cyprées environ). Outre trois bracelets, l'un au bras droit, l'autre au poignet droit et le troisième au poignet gauche, il portait un collier dont une partie des grains et plusieurs pendeloques fortement teintées d'ocre se voyaient encore en place sur la poitrine. Le reste des coquilles se trouvaient dans le bassin [...] Tout autour du crâne de l'enfant, et à hauteur des temporaux plus particulièrement, nous avons observé un tel amas de littorines et cyprées percées, que la possibilité d'une résille s'imposa de suite à notre esprit [...] Le dégagement du petit crâne nous permit de constater et de faire constater que le même amas de coquilles percées se retrouvait à la face postérieure du crâne " (Péquart et Péquart 1929, p. 17). L'existence de parure corporelle en coquillage ne saurait être totalement remise en cause dans les sépultures de Téviec et d'Hoëdic, mais nous avons déjà discuté des interprétations aujourd'hui proposées concernant de telles concentrations de petites coquilles (ou ailleurs de canines percées) autour de restes d'enfants dans les sépultures mésolithiques d'Europe occidentale.

Au final, les propos lyriques des époux Péquart mettent bien en valeur le rôle de toutes ces ornementations dans la mise en scène des corps des défunts, lors de leur mise au tombeau. En revanche, le texte est tellement empreint de présupposés et d'imprécision qu'il est bien difficile de statuer sur la nature exacte ou le statut des objets ainsi décorés. Faute de relevés suffisamment précis, les photographies nous sont rarement d'un grand secours. Nous nous sommes donc limités à mettre en exergue les éléments de ce discours qui entrent en résonnance avec nos propres observations au sein des tombes mésolithiques de La Vergne. Gageons que ces dernières n'auraient pas été décrites de façons très différentes de celles de Téviec ou d'Hoëdic, en ce qui concerne la parure, si elles avaient été fouillées à cette même époque et par les mêmes auteurs.

Il est un dernier point à prendre en compte. Des sépultures mésolithiques de La Vergne nous ne connaissons que ce qui en subsiste au-dessous d'un niveau du sol préhistorique aujourd'hui totalement arasé. Celles de Téviec et d'Hoëdic furent aménagées au sein d'amas coquillers. Elles s'insèrent et sont parfois remblayées avec des rejets domestiques où la découverte de coquilles percées n'est pas exceptionnelle (Kayser 1990, Dupont 2011, Dupont et al. 2010). Ce problème est particulièrement bien illustré par le site portugais de Moita do Sebastiao où la majorité des éléments de parure 
semble provenir au moins autant des couches environnantes que des inhumations proprement dites (Roche 1972, Figueiredo et al. 2016).

\section{Conclusion}

L'étude des objets de parure préhistorique reste essentiellement celle de pièces disjointes. C'est particulièrement vrai pour la parure en coquille d'origine marine. Chaque pièce livre ainsi maintes informations extrêmement précieuses sur son milieu de collecte, les distances de transport, les modes de façonnage, ou la façon dont elle aura été portée. Comme chaque pièce de vêtement, ces coquilles participent à la culture matérielle d'un groupe donné. Faisant fi de l'épaisseur chronologique des temps préhistoriques, certains voudront désormais voir dans leur assemblage, et leur composition, la marque de groupes «ethniques ». Depuis plus d'une dizaine d'années maintenant, on prend pleinement conscience que ces coquilles percées pourraient avoir orné bien d'autres objets de la vie quotidienne.

Alors que l'étude de chaque élément pris séparément mobilise des moyens techniques de plus en plus lourds, notre connaissance de la « parure » préhistorique, à proprement parler, reste donc encore quelque peu embryonnaire. La qualité de l'enregistrement effectué lors de la fouille des sépultures mésolithiques du site de La Vergne aura du moins permis d'identifier l'existence dans la tombe de maints objets ornés, distincts de la parure strictement associée aux corps des défunts. Dans de tels cas, ce type d'enregistrement mériterait assurément d'être plus largement utilisé. Peut-être aussi gagnerait-on encore en précision si, plus souvent, le fouilleur arrivait à dégager son esprit des seuls volumes du corps humain, se repérant sur la disposition des pièces d'ornementation pour tenter de dégager les contours de quelques-uns de ces objets. Mais ce n'est pas le cas le plus fréquent. Et par le passé, l'observation fut fréquemment biaisée par de nombreux présupposés.

Dans ces conditions, il paraît difficile d'étayer de plus amples comparaisons à nos propres déductions pour des ensembles qui restent dans tous les cas exceptionnels, de surcroît séparés par de larges plages chronologiques et de grandes distances géographiques. Les cimetières de Téviec et Hoëdic par exemple, les plus proches parmi les exemples cités, sont tout de même éloignés de plusieurs centaines de kilomètres et d'au moins trois millénaires des sépultures de La Vergne. Finalement, les éléments de comparaison les plus proches sur le plan géographique sont fournis par la seule sépulture assurément attribuable au Néolithique ancien du Centre-Ouest de la France. Datée de la charnière entre le $\mathrm{VI}^{\mathrm{e}}$ et le $\mathrm{V}^{\mathrm{e}}$ millénaire av. n.è., la sépulture double de Germignac en Charente est à peine plus récente que celles de Téviec et Hoëdic, comparée à l'ancienneté des tombes de La Vergne. Elle a également livré un nombre tout à fait exceptionnel d'objets de parure en coquillage (Gaillard et al. 1984, Laporte et Gomez 2001).

211 Avec 3200 pièces de parure recueillies, la sépulture néolithique de Germignac ne présente guère de comparaison à l'échelle nationale ; seule une sépulture découverte dans les années soixante dans le quartier de la Balance à Avignon pourrait lui être comparée de ce point de vue (Courtin et al. 1966). Cette dernière ne saurait être confondue avec une autre sépulture de même provenance récemment fouillée en laboratoire, qui fut également datée du début du V millénaire (Zemour et al. 2017). Quelques tombes à La Vergne, pour la période du Mésolithique, ou celle de Germignac, 
pour les débuts du Néolithique, comprennent dans les deux cas un nombre impressionnant de coquilles percées. Le fait que la matière première soit une coquille marine n'est sans doute pas anodin, loin de là, mais la forme et le degré de façonnage sont radicalement différents. Dans le premier cas, nous nous sommes même interrogés sur la pertinence du terme de matière première, ici remplacé par des entités naturelles que l'on expose, assemble et décline sur différents objets, peut-être à des fins esthétiques, peut-être à titre prophylactique, voire pour d'autres raisons encore.

La variété des formes de la parure mésolithique comme celle étudiée sur le site de La Vergne, issue d'une longue tradition ancrée dans le Paléolithique supérieur, apparaît ainsi encore plus clairement quand on l'oppose à la standardisation des produits finis qui commence à transparaître au travers des ensembles du tout début du Néolithique, dans l'ouest de la France. Car dans d'autres contextes contemporains, des disques somme toute assez semblables à ceux de Germignac ont été réalisés en calcaire, par exemple, sans que l'on puisse toujours faire la différence à l'œil nu. Un peu comme si l'importante transformation que subissent alors les tests coquilliers semblait les éloigner de leur milieu marin d'origine. Une opposition entre transformation de la matière et appropriation d'entités naturelles qui transparaît ainsi très clairement au travers du corpus de La Vergne, où maintes coquilles percées proviennent d'abord d'une collecte sélective sur le littoral.

Flavien Bambagioni, Vincent Miailhe et Karine Robin ont participé sur le terrain à la fouille du site de La Vergne. Henri Duday et Patrice Courtaud, anthropologues ont dirigé la fouille des sépultures en prenant en compte le mobilier associé. Ils sont les auteurs des relevés de terrain, dessins et photographies. Laurent Quesnel a repris en infographie l'ensemble des relevés de terrain sous l'œil expert d'Henri Duday. Jean-Yves Lefeuvre, photographe, a pris certains des clichés présentés. Catherine Dupont et Yves Gruet ont réalisé l'analyse des mollusques marins (détermination, quantification, observation des usures naturelles et de leur évolution taphonomique). Catherine Dupont a positionné les échelles sur l'ensemble des clichés des coquilles et de leurs traces associées. Luc Laporte et Catherine Dupont ont étudié les stigmates anthropiques observés sur les coquilles de leurs techniques de façonnage à leurs intensités. Luc Laporte a reconstitué la distribution spatiale des éléments de parure. Ce texte a été rédigé par Luc Laporte et Catherine Dupont à l'exception de la partie "Les coquilles marines: détermination, description et lieu probable de collecte »écrite par Catherine Dupont et Yves Gruet. Nous tenons également à remercier Chantal Leroyer de nous avoir aidés dans la calibration et la recherche de données climatiques associées à la phase d'occupation du site de La Vergne, ainsi que les relecteurs de la revue Gallia Préhistoire pour leurs remarques constructives.

\section{BIBLIOGRAPHIE}

Albrethsen S.E., Petersen E.B. 1977 - Excavation of a Mesolithic cementery at Vedaek, Denmark, Acta Archeologica, 47, p. 1-28. 
Allard J., Chaumillon E., Poirier C., Sauriau P.G., Weber 0. 2008 - Evidence of former Holocene sea level in the Marennes-Oléron Bay (French Atlantic coast). C. R. Geoscience, 340, p. 306-314.

André X. 1986 - Élaboration et analyse des cartes bathymétriques détaillées du proche plateau vendéocharentais. Reconstitution des paléorivages de la transgression holocène, thèse de doctorat, Université de Bordeaux I, inédit, $274 \mathrm{p}$.

Babin C., Glémarec M. 1971 - Écologie et paléoécologie des bivalves marins des sédiments meubles, Haliotis, 1.2, p. 105-125.

Bailey G., Parkington J. 1988 - The archaeology of prehistoric coastlines: an introduction, in Baily G., Parkington J. (dir.), The archaeology of prehistoric coastlines, Cambridge, Cambridge University Press, p. 1-20.

Barge H. 1982 - Les parures du Néolithique ancien au début de l'âge des métaux en Languedoc, Paris, CNRS édition, $396 \mathrm{p}$

Barge-Mahieu H. 1991 - Objets de parure : fiche coquillages néolithiques, Cahier. IV-2.2, in Barge-Mahieu H, Bellier C, Bott S, Cattelain P. (dir.), Fiches typologiques de l'industrie osseuse préhistorique, Cahier IV, Aix en Provence : Publication de l'Université de Provence.

Barge-Mahieu H., Taborin Y. 1991 - Objets de parure : fiche générale des objets de parure, Cahier. IV-0, in Barge-Mahieu H, Bellier C, Bott S, Cattelain P. (dir.), Fiches typologiques de l'industrie osseuse préhistorique, Cahier IV, Aix en Provence, Publication de l'Université de Provence.

Barusseau J.P. 1973 - Évolution du Plateau continental rochelais (Golfe de Gascogne) au cours du Pléistocène terminal et de l'Holocène, thèse d'état, Université de Bordeaux, inédit, 363 p.

Bar-Yosef Mayer DE. Groman-Yaroslavski I., Bar-Yosef O., Hershkovitz I., Kampen-Hasday A., Vandermeersch B., Zaidner Y., Weinstein-Evron M. 2020 - On holes and strings: Earliest displays of human adornment in the Middle Palaeolithic. PLoS ONE, 15(7), p. 1-13.

Bernier P., Gruet Y. 2011 - Environnement littoral. Sédimentation et Biodiversité de l'Estran. Île de Noirmoutier (Vendée), Lyon, Documents des Laboratoires de Géologie (HS 10), 163 p.

Binant P. 1991 - La préhistoire de la mort. Les premières sépultures en Europe, Paris, Éditions Errance, $170 \mathrm{p}$.

Bishop G.A. 1975 - Traces of Predation, in Frey R.W. (dir.), The Study of Trace Fossils, New York, Springer-Verlag, p. 261-281.

Boekshote N. 1966 - Shell borings of sessile epibiontic organisms as paleological guides, Paleogeography, Paleoclimats, Paleoecology, 2, p. 33-379.

Boulestin B. 2016 - Les sépultures mésolithiques de Téviec et Hoedic : revisions bioarchéologiques, Oxford, Archeopress, 307 p.

Borić D. 2016 - Deathways at Lepenski Vir: patterns in mortuary practice, Belgrade, Serbian Archaeological Society, $567 \mathrm{p}$

Borić D., French C.A.I., Stefanović S., Dimitrijević V., Cristiani E., Gurova M., Antonović D., Allué E.A., Filipović D. 2014 - Late Mesolithic lifeways and deathways at Vlasac (Serbia), Journal of Field Archaeology, 39.1, p. 4-31.

Boric D., Cristiani E. 2016 - Social networks and connectivity among the Palaeolithic and Mesolithic foragers of the Balkans and Italy, in Krauss R., Floss H. (dir.) Southeast Europe Before Neolithisation: Proceedings of the International Workshop within the Collaborative Research Centres SFB 
1070 “RESSOURCENKULTUREN”, Schloß Hohentübingen, 9th of May 2014, Tübingen: Universität Tübingen, RessourcenKulturen, vol. 1. p. 73-112.

Bugajska K., Guminski W. 2016 - How many steps to heaven? Loose human bones and secondary burials at Dudka and Szczepanki, the Stone Age foragers' sites in Masuria, N-E Poland, in Meller H., Grünberg J. M., Gramsch B., Larsson L., Orschiedt J. (dir.), Mesolithic burials - rites, symbols and social organisation of early postglacial communties. International Conference Halle (Saale), Germany, 19th-21st September 2013, Halle Saale, Landesmuseum für Vorgeschichte Halle, p. 511-544

Butrimas A. 2016 - Birzulis lake islands Donkalnis and Spiginas Mesolithic cemeteries (West Lithuania), in Meller H., Grünberg J. M., Gramsch B., Larsson L., Orschiedt J. (dir.), Mesolithic burials - rites, symbols and social organisation of early postglacial communties. International Conference Halle (Saale), Germany, 19th-21st September 2013, Halle Saale, Landesmuseum für Vorgeschichte Halle, p. 193-218.

Cabral J.P., Martins J.M.S. 2016 - Archaeological Glycymeris glycymeris shells perforated at the umbo: Natural or man-made holes? Journal of Archaeological Science: Reports, 10, p. 474-482.

Cabral J.P., Monteiro-Rodrigues S. 2015 - Orifícios de predação em conchas de moluscos marinhos. Um modelo experimental para o estudo de perfurações em conchas arqueológicas La Investigación Arqueomalacológica en la Península Ibérica: Nuevas Aportaciones, in GutiérrezZugasti1 I., Cuenca Solana D., Gonzalez Morales M.R. (dir.), Actas de la IV Reunion de Arqueomalacologia de la Peninsula Iberica, Santander, 22-24 mai 2014, Santander, Nadir Ediciones, p. 241-250.

Capitan L., Peyrony D. 1928 - La Madeleine, Paris, Nourry, Publications de l'Institut international d'anthropologie, $125 \mathrm{p}$.

Carbonel P., Dartevelle H., Evin J., Gruet Y., Laporte L., Marambat L., Tastet J.P., Vella C., Weber 0.1998 - Évolution paléogéographique de l'estuaire de la Charente au cours de l'Holocène, in Laporte L. (dir.), Estuaire de la Charente de la Protohistoire au Moyen Âge, Paris, Document d'Archéologie Française (série Grands Travaux), p. 15-25.

Chavan A. 1996 - Les mollusques et leur détermination, Paris, Société d'édition d'enseignement supérieur, $94 \mathrm{p}$.

Cauwe N. 2005 - L'importance des morts au Mésolithique, Anthropologica et Prsehistorica, 116, p. $149-164$.

Claassen C. 1998 - Shells. Cambridge manuals in archaeology, Cambridge, Cambridge University Press, 266 p.

Courtau P., Duday H. 1995 - Découverte d'une nécropole mésolithique à La Vergne (CharenteMaritime), Bulletins et Mémoires de la Société d'Anthropologie de Paris, 7, 3-4, p. 181-184.

Courtaud P., Duday H., Martin H., Robin K., 1999 - La nécropole mésolithique de La Vergne (Charente-Maritime, France), in Bintz P., Thevenin A. (dir.), L'Europe des derniers chasseurs Épipaléolithique et Mésolithique 5e colloque international UISPP, Grenoble, 18-23 septembre 1995, Paris, Éditions du CTHS, p. 8287-292.

Courtin J., Gagniere S., Germand L., Granier J. 1966 - Une sépulture néolithique en fosse à Avignon, Bulletin de la Société d'Etudes des Sciences Naturelles du Vaucluse, p.747-760.

Crothers J. H. 1973 - On variation in Nucella lapillus (L.): shell shape in populations from Pembrokeshire, South Wales, Journal of Molluscan Studies, 40(4), p. 319-320.

d'Errico F., Jardon-Giner P., Soler Mayor B. 1993 - Critères à base expérimentale pour l'étude des perforations naturelles et intentionnelles sur coquillages, in Anderson-Gerfaud P.C., Beyries 
S., Otte M., Plisson H. (dir.), Traces et fonction : gestes retrouvés, Colloque international de Liège, Liège, CRA et Université de Liège (ERAUL 50), p. 243-254.

d'Errico F., Vanhaeren M. 2000 - Mes morts et les morts de mes voisins. Le mobilier funéraire de l'Aven des Iboussières et l'identification des marqueurs culturels à l'Epipaléolithique, in Cupillard C., Richard A. (dir.), Les derniers chasseurs-cueilleurs d'Europe occidentale (13000-5500 av. J.C.). Actes du colloque international de Besançon, octobre 1998, Besançon, Presses Universitaires Franc-Comtoises, p. 325-342.

Dance S.P. 1993 - Les coquillages, Paris, Bordas (L'œil Nature), 256 p.

Dautzenberg PH. 1913 - Atlas de poche des coquilles des côtes de France (Manche, Océan, Méditerranée), communes, pittoresques ou comestibles, Paris, LHOMME : Librairie du Muséum de Paris, 152 p.

Duday H., Courtaud P. 1998 - La nécropole mésolithique de La Vergne (Charente-Maritime), in Guilaine J. (dir.) Sépultures d'Occident et genèses des mégalithismes (9000-3500 avant notre ère), Séminaire du Collège de France, Paris, Edition Errance, p. 27-37.

Duday H., Courtaud P. Dujardin V., Martin H., Robin K. 1996 - La Vergne : La Grande Pièce (Charente-Maritime), Complément au D.F.S. de sauvetage urgent. A.F.A.N, SRA Poitou- Charente, inédit, $33 \mathrm{p}$.

Duday H., Courtaud P., Robin K., Dujardin V., Gruet Y., Gourand G., Martin H., San JuanFoucher C. 1998 - La Vergne : La Grande Pièce (Déviation de Saint-Jean-d'Angély, CharenteMaritime), Bulletin de la Société préhistorique française, 95 (3), p. 423-435.

Dupont C. 1998 - La malacofaune des sépultures de La Grande Pièce' (La Vergne, Charente- Maritime), Mémoire de Diplôme d'Étude Approfondie, Institut de Préhistoire et de Géologie du Quaternaire, Université Bordeaux I, inédit, $100 \mathrm{p}$.

Dupont C. 2006 - La malacofaune de sites mésolithiques et néolithiques de la façade atlantique de la France : Contribution à l'économie et à l'identité culturelle des groupes concernés, Oxford, Archeopress (British Archaeological Reports, International Series 1571), 439 p.

Dupont C. 2011 - Étude complémentaire : les invertébrés marins du « concheiro » de Toledo (Lourinhã, Portugal), in Araújo A.C. (dir.), O concheiro de Toledo no contexto do Mesolítico inicial di litoral da Estremadura (Trabalhos de Arqueologia 51), p. 185-227.

Dupont C. C., 2019 - Archaeological evidence for collecting empty shells along the French Atlantic coast: A major activity for coastal populations, Journal of Ethnobiology, 39(2), p. 223-239.

Dupont C., Gruet Y. 2000 - Variations morphologiques de mollusques gastropodes (Nucella lapillus et Hinia reticulata) : intérêts pour l'archéologie, Revue d'Archéométrie, 24, p. 53-61.

Dupont C., Marchand G., Carrion Y., Desse-Berset N., Gaudin L., Gruet Y., Marguerie D., Oberlin C. 2010 - Beg-an-Dorchenn : une fenêtre ouverte sur l'exploitation du littoral par les peuples mésolithiques du sixième millénaire dans l'ouest de la France, Bulletin de la Société Préhistorique Française, 107-2, p. 227-290.

Dupont C., Tresset A., Desse-Berset N., Gruet Y., Marchand G., Schulting R. 2009 Harvesting the seashores in the Late Mesolithic of North-Western Europe: a view from Britanny, Journal of World Archaeology, 22/2, p. 27-37.

Dupont C., Laporte L., Courtaud P., Duday H., Gruet Y. 2014 - Personal shell adornments? at La Vergne (Charente-Maritime, France): from acquisition to wear, in Szabó K., Dupont C., Dimitrijevic V., Gastélum Gómez L. G., Serrand N. (dir.), Archaeomalacology: Shells in the Archaeological Record. Proceedings of the 11th ICAZ International Conference. Paris - Archaeomalacology 
Working group, 23-28 August 2010, France, Oxford, Archeopress, (BAR International Series 2666), p. 43-52.

Dyke S. 1996 - Marine molluscs as indications of environmental change in glaciated north America and Greenland during the last 18000 years, Géographie et quaternaire, 50 (2), p. 125-184.

Einwögerer T., Friesinger H., Händel M., Neugebauer-Marech C., Simon U., Teschler-Nicola M. 2006 - Upper Paleolithic infant burials. Decorations on the bodies of new borns indicate that they were probably important in their community, Nature, 444, p. 285.

Figueiredo O., Umbelino C., Bicho N. 2016 - Mortuary variability at Moita do Sebastiao et Cabeco da Amoreira (Muge, Central Portugal), in Meller H., Grünberg J. M., Gramsch B., Larsson L., Orschiedt J. (dir.), Mesolithic burials - rites, symbols and social organisation of early postglacial communties. International Conference Halle (Saale), Germany, 19th-21st September 2013, Halle Saale, Landesmuseum für Vorgeschichte Halle, p. 673-681.

Fischer A. (dir.) 1995 - Man and sea in the Mesolithic: coastal settlement above and below present sea level, Oxford, Oxbow Books, $440 \mathrm{p}$.

Fretter V., Graham A. 1976 - The prosobranch molluscs of Britain and Danmark, Part.1Pleurotomariacea, Fissurelacea and Patellacea, Journal of Molluscan Studies, 42 (1), p. 1-22.

Fretter V., Graham A. 1978 - The prosobranch molluscs of Britain and Danmark, Part.3Neritacea, Viviparacea, Valvatacea, terrestrial and freshwater Littorinacea and Rissoacea. Journal of Molluscan Studies, 44 (5), p. 101-152.

Fretter V., Graham A. 1981 - The prosobranch molluscs of Britain and Danmark, Part.6Cerithiacea, Stombacea, Hipponicacea, Lamellariacea, Cyperaeacea, Euspiracea, Tonnacea, Heteropoda. Journal of Molluscan Studies, 47 (9), p. 285-360.

Fretter V., Graham A. 1985 - The prosobranch molluscs of Britain and Danmark, Part.8Neogasteropoda. Journal of Molluscan Studies, 51 (15), p. 435-556.

Gaillard J., Taborin Y., Gomez de Soto J. 1984 - La tombe néolithique de Germignac. GalliaPréhistoire, 27, p. 97-119.

García-Artola A., Séphan P., Cearreta A., Koppe R.E., Khan N.S., Horton B.P. 2018 - Holocene sea-level database from the Atlantic coast of Europe. Quaternary Science Reviews, 196, p. 177-192.

Gehlen B. 2016 - No future? No past? Mesolithic heritage in Neolithic burials, in Meller H. Grünberg J. M., Gramsch B., Larsson L., Orschiedt J. (dir.), Mesolithic burials - rites, symbols and social organisation of early postglacial communties. International Conference Halle (Saale), Germany, 19th-21st September 2013, Halle Saale, Landesmuseum für Vorgeschichte Halle, p. 827-850.

Hinschberger F, Pauvret, RB. 1968 - Les fonds sous-marins de l'Iroise et de la baie de Douarnenez (reconstitution d'un réseau hydrographique immergé), Norois, 15, p. 213-225.

Hubendick B. 1947 - Die Verbreitungsverhältnisse der limnischen Gastropoden in Südschweden, Zoologiska Bidräg, Uppsala, 24, p. 419-559.

Jaeckel S. 1952 - Zur Oekologie der Molluskenfauna in der westlichen Ostsee, Schriften des Naturwissenschaftlichen Vereins für Schleswig-Holstein, 26, p. 18-50.

Jeunesse C. 1995 - Les groupes régionaux du Rubané (Rhin et Bassin parisien) à travers les pratiques funéraires, Gallia Préhistoire, 37, p. 115-154.

Jeunesse C. 1997 - Pratiques funéraires en Néolithique ancien : sépultures et nécropoles danubiennes (5500-4900 av. J.-C.), Paris, Éditions Errance, 167 p. 
Julien M., Averbough A., Ramseyer D., Bellier C., Buisson D. Cattelain P., Patou-Mathis M., Provenzano N. (dir.) 1999 - Préhistoire d'Os : Recueil d'études sur l'industrie osseuse préhistorique offert à H. Camps-Fabrer, Aix en Provence, Publication de l'Université de Provence, 336 p.

Kayser 0. 1990 - Sur les rites funéraires des chasseurs-collecteurs d'Europe de l'Ouest et du Nord-Ouest à la fin du Mésolithique, Revue Archéologique de l'Ouest, Supplément 2, p. 75-80.

Kayser 0.1989 - Le mésolithique des îles bretonnes, Bulletin de l'AMARAI, 2, p. 1-2.

Kaufman D., McKay N., Routson C., Erb M., Dätwyler C., Sommer P.S., Heiri O., Davis B. 2020 - Holocene global mean surface temperature, a multi-method reconstruction approach, Scientific Data, 7(201).

Laporte L. 2014 - Estudio del ajuar funerario de los entierros humanos, in Politis G., Gutiérrez M.A., Scabuzzo C. (dir.), Estado actual de las investigaciones en el sitio arqueológico Arroyo Seco 2 (partido de Tres Arroyos, provincia de Buenos Aires, Argentina), Buenos Aires, INCUAPA, CONICET, UNICEN (Serie Monográfica 5), p. 393-415.

Laporte L., Dupont C. 2019 - Personal adornments and objects of ornamentation: two case studies from hunter-gatherer burials, in France (La Vergne) and Argentina (Arroyo Seco II), PaleoAnthropology, 9-10, p. 156-176.

Laporte L., Gomez de Soto J. 2001 - Germinac et Lamérac : perles discoïdes et anneaux- disques dans le Centre-Ouest de la France, Revue Archéologique de l'Ouest, 18, p. 13-26.

Lévi-Strauss C., 1962 - Le totémisme aujourd'hui, Mythes Et Religions, Paris, Presses Universitaire de France, 42, $154 \mathrm{p}$.

Marchand G., Schulting R. 2019 - Chronologie du second Mésolithique dans le Nord-Ouest de la France, in Arbogast R.M., Griselin S., Jeunesse C., Séara F. (dir.), Le second Mésolithique, des Alpes à l'Atlantique (VII ${ }^{\circ} V^{\circ}$ millénaire). Table ronde internationale, Strasbourg, 3 et 4 novembre 2015, Mémoire d'Archéologie du Grand-Est, 3, p. 109-125.

Martin C., Ménot G., Thouveny N., Peyron O., Andrieu-Ponel V., Montade V. Davtian N., Reille M., Bard E. 2020 - Early Holocene Thermal Maximum recorded by branched tetraethers and pollen in Western Europe (Massif Central, France), Quaternary Science Reviews, 228, 106-109.

May F. 1986 - Les Sépultures préhistoriques : étude critique. Paris, CNRS Ed., 286 p.

Moore P. G. 1984 - Shell Shape in Living and Fossil ('25ft' Beach) Dog-whelks, Nucella lapillus (L.), from the Isle of Cumbrae. Scotland, Glasgow Naturalist, 21 (1), p. 81-92.

Mougne C. 2015 - Exploitation et utilisation des invertébrés marins durant la Protohistoire sur le territoire continental et littoral Manche-Atlantique français, thèse de doctorat, Université Rennes 1 , inédit, $707 \mathrm{p}$.

Newell R.R., Kielman D., Constandse-Westermann T.S., Van der Sanden W.A.B., Van Gijn A. 1990 - An inquiry into the ethnic resolution of Mesolithic regional groups: the study of their decorative ornaments in time and space. Leiden, Brill, $488 \mathrm{p}$.

Olivier P.H. 1975 - Les coquillages marins du monde en couleur, Bruxelles, Elsevier Séquoia, 324 p.

Péquart M., Péquart S.-J. 1929 - La nécropole mésolithique de Téviec. (Morbihan. Nouvelles découvertes), L'Anthropologie, 39, p. 373-400.

Péquart M., Péquart S.-J. 1954 - Hoëdic, deuxième station-nécropole du Mésolithique côtier Armoricain, Anvers, De Sikkel, 93 p. 
Péquart M., Péquart S.-J., Boule M., Vallois H. 1937 - Téviec : station nécropole mésolithique du Morbihan, Paris, Masson (Archives de l'Institut de Paléontologie humaine 18), 227 p.

Perlès C. 2018 - Ornaments and Other Ambiguous Artifacts from Franchthi: Volume 1, The Palaeolithic and the Mesolithic (Excavations at Franchthi Cave, Greece) Excavations at Franchthi Cave, Greece (Book 15) Indiana University Press, Indiana, 326 p.

Petersen P.V. 2016 - Papooses in the Mesolithic? A reinterpretation of tooth and snail shell ornaments found in grave 8 at Bogebakken and other Mesolithic burials, in Meller H., Grünberg J. M., Gramsch B., Larsson L., Orschiedt J. (dir.), Mesolithic burials - rites, symbols and social organisation of early postglacial communties. International Conference Halle (Saale), Germany, 19th-21st September 2013, Halle Saale, Landesmuseum für Vorgeschichte Halle, p. 109-124.

Phillips R. 1987 - Coquillages et algues marins, Bordas, Paris, 160 p.

Pinot J.P. 1973 - Morphologie littorale et sous-marine, Igahrun, Nantes, 80 p.

Pinot J.P. 1998 - La gestion du littoral. Tome I- Littoraux tempérés : côtes rocheuses et sableuses : Tome II - Littoraux tempérés : littoraux vaseux et embouchures, Paris, Éditions Institut océanographique, $759 \mathrm{p}$.

Poppe T., Goto Y. 1991 - European Seashells: Polyplacophora, Caudofoveata, Solenogastra, Gasteropoda, Germany, Verlag Christa Hemmen, vol.1, 352 p.

Poppe T., Goto Y. 1993 - European Seashells: Scaphopoda, Bivalvia, Cephalopoda, Germany, Verlag Christa Hemmen, vol.2, 221 p.

Reimer P., Austin W., Bard E., Bayliss, A., Blackwell, P., Bronk Ramsey C., Butzin M., Cheng H., Edwards R., Friedrich M., Grootes P., Guilderson T., Hajdas I., Heaton T., Hogg A., Hughen K., Kromer B., Manning S., Muscheler R., Palmer J., Pearson C., van der Plicht J., Reimer R., Richards D., Scott E., Southon J., Turney C., Wacker L., Adolphi F., Büntgen U., Capano M., Fahrni S., Fogtmann-Schulz A., Friedrich R., Köhler P., Kudsk S., Miyake F., Olsen J., Reinig F., Sakamoto M., Sookdeo A., Talamo S. 2020 - The IntCal20 Northern Hemisphere radiocarbon age calibration curve (0-55 cal kBP), Radiocarbon, 62.

Regrain R. 1980 - Géographie physique et télédétection des marais charentais, Thèse de doctorat, Université de Brest, inédit, 512 p.

Rigaud S. 2011 - La parure : traceur de la géographie culturelle et des dynamiques de peuplement au passage Mésolithique-Néolithique en Europe, Thèse de doctorat, Université de Bordeaux I, inédit, 470 p.

Rigaud S. 2013 - Les objets de parure associés au dépôt funéraire mésolithique de Große Ofnet (Allemagne): implications pour la compréhension de l'organisation sociale des dernières sociétés mésolithiques du Jura Souabe, Anthropozoologica, 48 (2), p. 207-230.

Rigaud S. 2014 - Pratiques ornementales des premieres communautés agro-pastorales de Baviere (Allemagne) : Intégration? Acculturation ? Convergence ? Nouveaux apports de la nécropole de Essenbach-Ammerbreite [Personal ornaments of the first agro-pastoral societies in Bavaria (Germany): Integration? Acculturation? Convergence? New insights from EssenbachAmmerbreite cemetery]. Anthropologie (Brno) 52, 2: 207-227.

Rigaud S., Vanhaeren M., Quefelec A., Le Bourdon G., d'Errico F. 2014 - The way we wear makes the difference: residue analysis applied to Mesolithic personal ornaments from Hohlenstein-Stadel (Germany), Archaeological and Anthropological Sciences, 6 (2), p. 133-144. 
Rivière E. 1905 - Sur l'emploi des dentales aux temps préhistoriques comme ornement, Bulletin de la Société Préhistorique Française, 2, p. 286-289.

Roche J. 1972 - Le gisement mésolithique de Moita do Sebastiao, Muge, Portugal, Lisboa, Instituto de Alta Cultura, $183 \mathrm{p}$.

Rolin M.F. 1971 - Etat des restes de Bivalves dans les thanatocénoses et relations avec les conditions de formation : les cassures, Thèse de doctorat, Université de Paris, inédit, 77 p.

Rozoy J.G. 1978 - Les derniers chasseurs. L'Epipaléolithique en France et en Belgique. Essai de synthèse. Charleville, Société Archéologique Champenoise, 1256 p.

Sabelli B. 1981 - Le guide des coquillages, Paris, Nathan, 512 p.

Schulting R.J. 1996 - Antlers, bone pins and flint blades: the Mesolithic cemeteries of Téviec and Hoëdic. Brittany, Antiquity, 70, p. 335-350.

Schulting R. J., Blockley S. M., Bocherens H., Drucker D., Richards M. 2008 - Stable carbon and nitrogen isotope analysis on human remains from the Early Mesolithic site of La Vergne (Charente-Maritime, France), Journal of Archaeological Science, 35, p. 763-772.

Séfériadés M. 1995 - La route des spondyles de la Méditerranée à la Manche, in Otte M. (dir.), Nature et Culture, Actes du colloque de Liège, 13-17 décembre 1993 (Études et recherches archéologiques de l'Université de Liège 68), p. 289-356.

Shackleton J.C. 1988 - Reconstitution past shorelines as an approach to determining factors affecting shellfish collecting in the prehistoric pas, in Baily G., Parkington J. (dir.), The archaeology of prehistoric coastlines, Oxford, Cambridge University Press, p. 11-21.

Stéphan P., Goslin J. 2014 - Évolution du niveau marin relatif à l'Holocène le long des côtes françaises de l'Atlantique et de la Manche : réactualisation des données par la méthode des « sealevel index points ", Quaternaire, 25/4, p. 295-312.

Taborin Y. 1971 - La parure en coquillage de l'Epipaléolithique au bronze ancien en France, Thèse de doctorat, Université de Paris 1, inédit, $160 \mathrm{p}$.

Taborin Y. 1974 - La parure en coquillage de l'Epipaléolithique au bronze ancien en France, Paris, CNRS Éditions (Gallia Préhistoire 17), p. 101-179; p. 307-417.

Taborin Y. 1982 - La parure des morts, Archeologia, 66, p. 46-51.

Taborin Y. 1984 - La tombe néolithique de Germignac (Charente-Maritime), Les disques percés en tests de lamellibranches, Gallia Préhistoire, 27(1), p. 98-108.

Taborin Y. 1993 - La parure en coquillage au Paléolithique. Paris, Éditions du CNRS (Gallia Préhistoire supplément XXIX), 538 p.

Taborin Y. 2004 - Langage sans parole. La parure aux temps préhistoriques, La Maison des Roches éditeur, Paris, $216 \mathrm{p}$.

Tebble N. 1966 - British Bivalve Seashells. A handbook for identification, Londres, Trustees of The British Museum Natural History, 213 p.

Testart A. 2004 - Les morts d'accompagnement. La servitude volontaire I. Paris, Éditions Errance, 264 p.

Trinkaus E., Buzhilova A., 2018 - Diversity and differential disposal of the dead at Sunghir. Antiquity, 92 (361), 7-21. doi:10.15184/aqy.2017.223 
Vanhaeren M. 2002 - Les fonctions de la parure au Paléolithique supérieur : de l'individu à l'unité culturelle, Thèse de doctorat, Université de Bordeaux 1, $355 \mathrm{p}$

Vanhaeren M., d'Errico F., 2001 - La parure de l'enfant de la Madeleine (fouilles Peyrony). Un nouveau regard sur l'enfance au Paléolithique supérieur, Paléo, 13, p. 201-240.

Vanhaeren M., d'Errico F. 2011 - L'émergence du corps paré, Civilisations, 59-2, p. 59-86.

Verneau R. 1906 - Les grottes de Grimaldi, Monaco, Imprimerie de Monaco (Anthropologie II-I), $207 \mathrm{p}$.

Vigneaux M., 1975 - Guides géologiques régionaux : Aquitaine occidentale. Paris, Masson, 224 p.

Villotte S. 2008 - Enthésopathies et activités des hommes préhistoriques-Recherche méthodologique et application aux fossiles européens du Paléolithique supérieur et du Mésolithique. 2008. Thèse de doctorat.

WoRMS, 2020 - World Register of Marine Species, URL:http://www.marinespecies.org/.2018.

Wye K.R. 1990 - Coquillages, Paris, Arthaud, 187 p.

Zemour A, Binder D., Bonnardin S., D'Ovidio A.-M., Goude G., Gourichon L., Pradeau J.-V., Sorin-Mazouni S., Bromblet P., Buchet L., Cotto K.-Y., Sénépart I. 2017 - Laboratory

Excavation of a Neolithic Grave from Avignon-La Balance-Ilot P (France): Burial Practices and Garment Reconstruction, Journal of Field Archaeology, 42(1), p. 54-68.

Zilhão J. 2007 - The emergence of ornametns and art: an archaeological perspective on the origin of behavioral modernity, Journal of Archaeological Research, 15, p. 1-54.

\section{NOTES}

1. Destinée à une monographie qui n'a finalement jamais vu le jour, cette étude a d'abord été livrée en 2005 sous la forme de deux chapitres distincts, puis actualisée et adaptée aux standards de la revue Gallia Préhistoire, en 2019.

2. La rédaction de cette partie du texte en introduction (1.3 à 1.5) a été finalisée en 2005 et, quinze ans plus tard, ne nous a pas semblé devoir être sensiblement modifiée. Ces textes furent alors rédigés sans avoir connaissance de travaux qui n'étaient pas encore parus (souvent, ni même conçus) mais qui, en revanche, seront largement cités dans la partie concernant les discussions, rendant compte ainsi de la démarche suivie par les auteurs.

\section{RÉSUMÉS}

Les sépultures mésolithiques de La Vergne ont livré 3297 coquilles, pour la plupart d'origine marine et recueillies échouées sur un estran alors situé à moins de 30 kilomètres. La plupart des coquilles sont percées, par le biais d'une action anthropique lorsque la nature n'avait pas déjà fait son œuvre. Leurs traces d'usures, comme la place de chacune de ces coquilles dans les trois 
principales sépultures, permettent de restituer nombre d'objets en matière périssable qui ont participé à la mise en scène des funérailles, ainsi que quelques éléments de parure corporelle. Après avoir décrit à la fois, la recherche des coquilles sur la grève, leur utilisation et leur dépôt avec les dépouilles, le caractère exceptionnel de cette découverte est ensuite discuté dans un cadre plus large, sur le plan géographique à l'échelle de l'Europe occidentale, et sur le plan chronologique en les comparant localement à quelques tombes des tout premiers agriculteurs de la région qui ont livré de très nombreuses pièces de parure façonnées à partir de la même matière première.

The Mesolithic burials of La Vergne delivered 3,297 marine shells, mostly from marine environments and collected on a foreshore located at less than 30 kilometers away. Most of the shells are pierced, through an anthropic action when nature had not already done its work. Traces of wear, as well as for many other detailed observations, were compared with the place of each item in the grave. These data, for example, make it possible to identify many perishable objects and some elements of body adornment that participated in the staging of the funeral. After describing both, the search for the shells on the beach, their use and their deposition with the mortal remains, the exceptional nature of this discovery is discussed in a broader context, geographically on a Western European scale, and chronologically by comparing them locally to some of the graves of the region's first farmers, who delivered many pieces of adornment made from this same raw material.

\section{INDEX}

Mots-clés : Mésolithique, parure, coquilles, cimetière, sépulture

Keywords : Mesolithic, ornament, shell, graveyard, burial

\section{AUTEURS}

\section{LUC LAPORTE}

UMR 6566 CReAAH « Centre de Recherche en Archéologie, Archéosciences, Histoire » CNRS, Université Rennes, Campus Beaulieu - France

\section{CATHERINE DUPONT}

UMR 6566 CReAAH « Centre de Recherche en Archéologie, Archéosciences, Histoire » CNRS, Université Rennes, Campus Beaulieu - France

\section{YVES GRUET}

Retraité de l'Université de Nantes, 58 rue Stendhal, 44300 Nantes, France

\section{PATRICE COURTAUD}

UMR 5199 PACEA, Université de Bordeaux, CNRS - France

\section{HENRI DUDAY}

UMR 5199 PACEA, Université de Bordeaux, CNRS - France 


\section{LAURENT QUESNEL}

UMR 6566 CReAAH « Centre de Recherche en Archéologie, Archéosciences, Histoire » CNRS, Université Rennes, Campus Beaulieu - France

JEAN-YVES LEFEUVRE

UMR 6566 CReAAH « Centre de Recherche en Archéologie, Archéosciences, Histoire » CNRS, Université Rennes, Campus Beaulieu - France

\section{FLAVIEN BAMBAGIONI}

Inrap Nouvelle-Aquitaine et Outre-Mer

\section{VINCENT MIAILHE}

Inrap Nouvelle-Aquitaine et Outre-Mer

\section{KARINE ROBIN}

Service archéologique départemental de la Charente-Maritime 